\title{
Advanced diagnostics and therapeutics for Alzheimer's disease
}

Citation for published version (APA):

Stevens, J. A. A. (2016). Advanced diagnostics and therapeutics for Alzheimer's disease. [Doctoral Thesis, Maastricht University]. Maastricht University. https://doi.org/10.26481/dis.20161028js

Document status and date:

Published: 01/01/2016

DOI:

10.26481/dis.20161028js

Document Version:

Publisher's PDF, also known as Version of record

\section{Please check the document version of this publication:}

- A submitted manuscript is the version of the article upon submission and before peer-review. There can be important differences between the submitted version and the official published version of record.

People interested in the research are advised to contact the author for the final version of the publication, or visit the DOI to the publisher's website.

- The final author version and the galley proof are versions of the publication after peer review.

- The final published version features the final layout of the paper including the volume, issue and page numbers.

Link to publication

\footnotetext{
General rights rights.

- You may freely distribute the URL identifying the publication in the public portal. please follow below link for the End User Agreement:

www.umlib.nl/taverne-license

Take down policy

If you believe that this document breaches copyright please contact us at:

repository@maastrichtuniversity.nl

providing details and we will investigate your claim.
}

Copyright and moral rights for the publications made accessible in the public portal are retained by the authors and/or other copyright owners and it is a condition of accessing publications that users recognise and abide by the legal requirements associated with these

- Users may download and print one copy of any publication from the public portal for the purpose of private study or research.

- You may not further distribute the material or use it for any profit-making activity or commercial gain

If the publication is distributed under the terms of Article $25 \mathrm{fa}$ of the Dutch Copyright Act, indicated by the "Taverne" license above, 


\section{ADVANCED DIAGNOSTICS AND THERAPEUTICS FOR ALZHEIMER'S DISEASE}

Jo Armand Albert STEvens 
Printed by: $\quad$ CPI-Koninklijke Wöhrmann - Zutphen

Cover design by: Jo Stevens

All rights reserved. No part of this book may be reproduced or transmitted in any form or by any means, without permission in writing, from the copyright holder.

Copyright (C) by J.A.A. Stevens, Maastricht, 2016

ISBN 978-94-6328-111-9 


\section{ADVANCED DIAGNOSTICS AND THERAPEUTICS FOR ALZHEIMER'S DISEASE}

\section{PROEFSCHRIFT}

ter verkrijging van de graad van doctor aan de Universiteit Maastricht, op gezag van de Rector Magnificus, Prof dr. Rianne M. Letschert volgens het besluit van het College van Decanen, in het openbaar te verdedigen op vrijdag 28 oktober 2016 om 10:00

door

\section{Jo Armand Albert SteVenS}




\section{Promotor:}

Prof. dr. M. de Baets

\section{Copromotors:}

Dr. M. Losen

Dr. P. Martinez-Martinez

\section{Beoordelingscommissie:}

Prof. dr. R. Hoet Chairman

Dr. M. Bauwens

Dr. F. van Leeuwen

Prof. dr. P. Parren Universiteit Leiden

Prof. dr. J. Walter University of Bonn

Financial support for the completion of this thesis was kindly provided by:

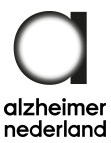


In herinnering aan Armand en Albert Voor mijn petekind Mats 



\section{CONTENTS}

1 Introduction $\quad \mathbf{1}$

1.1 Clinical and pathological features of Alzheimer's disease . . . . . . . . 2

1.2 Pathology and treatment options . . . . . . . . . . . . . . . 3

1.3 Diagnosis of AD . . . . . . . . . . . . . . . . . . . . . 6

1.4 Aims and outline. . . . . . . . . . . . . . . . . 8

References. . . . . . . . . . . . . . . . . . 10

2 Inflammation and Alzheimer's disease: lessons from immunotherapy $\quad 15$

2.1 The amyloid cascade hypothesis . . . . . . . . . . . . . . . . . . 17

2.2 Other factors in $A D$. . . . . . . . . . . . . . . . . . . . . 19

2.3 The immune system and $A D$. . . . . . . . . . . . . . . . . . . . . 19

2.3.1 The role of microglia in AD . . . . . . . . . . . . . . . 20

2.3.2 Complement and the inflammasome in AD . . . . . . . . . . . . 21

2.4 Effector mechanisms of $A \beta$ immunotherapy . . . . . . . . . . . . . . . 22

2.4.1 FcR mediated phagocytosis . . . . . . . . . . . . . . . 23

2.4.2 Peripheral sink . . . . . . . . . . . . . . . . . 24

2.4.3 Catalytic dissolution . . . . . . . . . . . . . . . . 25

2.5 Blood Brain Barrier. . . . . . . . . . . . . . . . . . . . . . 26

2.6 Clinical results of anti-A $\beta$ immunotherapy . . . . . . . . . . . . . . 27

2.6.1 Active immunization . . . . . . . . . . . . . . . . . 27

2.6.2 Passive immunization . . . . . . . . . . . . . . . . . 29

2.7 Additional safety concerns in immunotherapy . . . . . . . . . . . . . . 32

2.8 Conclusions and Future perspectives. . . . . . . . . . . . . . . . 33

References...................... . . 50

3 Isotype dependent effector mechanisms of immunotherapy in AD 73

3.1 Introduction . . . . . . . . . . . . . . . . . 75

3.2 Methods ......................... 76

3.2.1 Animal breeding . . . . . . . . . . . . . . . . 76

3.2.2 Antibody production . . . . . . . . . . . . . . . 76

3.2.3 Experimental setup and tissue processing . . . . . . . . . . . 77

3.2.4 Behavioral procedures . . . . . . . . . . . . . . . . 77

3.2.5 Immunofluorescence and histochemistry . . . . . . . . . . . . 79

3.2 .6 ELISA . . . . . . . . . . . . . . . . 80

3.2.7 Statistical analyses .................. 80 
3.3 Results . . . . . . . . . . . . . . . . . . . . . . . . . . . . 81

3.3.1 Immunotherapy with IgG1 and IgG2a 3D6 antibodies causes increased mortality of 5xFAD mice . . . . . . . . . . . . . 81

3.3.2 mlgG1 worsened cognitive deficits in the contextual fear conditioning test . . . . . . . . . . . . . 82

3.3.3 Preventive immunotherapy regimens decreased $A \beta$ plaque load . 85

3.3.4 Reduction in astrogliosis is isotype dependent . . . . . . . . . 87

3.4 Discussion . . . . . . . . . . . . . . . . . . . . . . . . . . . . 89

References. . . . . . . . . . . . . . . . . . . . . . 91

4 In vivo PET imaging in an AD model 95

4.1 Introduction . . . . . . . . . . . . . . . . . . 97

4.2 Material and Methods . . . . . . . . . . . . . . . . . . . 98

4.2 .1 Animals . . . . . . . . . . . . . . . . . . . . . . . 98

4.2 .2 Antibody labeling . . . . . . . . . . . . . . . . . . . 98

4.2.3 Imaging and data analysis . . . . . . . . . . . . . . . . 999

4.2 .4 Biodistribution . . . . . . . . . . . . . . . . . . . . . 99

4.2.5 Autoradiography, immunofluorescence and microscopy . . . . 100

4.2 .6 Statistical analysis . . . . . . . . . . . . . . . . . . 100

4.3 Results . . . . . . . . . . . . . . . . . . . . . . . . . . . . 101

4.3.1 Specific accumulation of ${ }^{89} \mathrm{Zr}$-bapineuzumab in the 5 XFAD brain in vivo . . . . . . . . . . . . . . . . . . . . . . . . 101

4.3.2 Early detection of ${ }^{89} \mathrm{Zr}$-bapineuzumab in young $5 x F A D$ mice . . . 102

4.3.3 Biodistribution analysis confirmed early age-specific uptake of ${ }^{89} \mathrm{Zr}$-bapineuzumab. . . . . . . . . . . . . . . 105

4.3.4 ${ }^{89} \mathrm{Zr}$-bapineuzumab co-localized with $A \beta$ plaques in the brain . . 107

4.4 Discussion . . . . . . . . . . . . . . . . . . . . . . . . 109

References. . . . . . . . . . . . . . . . . . . . . . 111

5 A comparison of three anti-amyloid antibodies with immuno-PET $\quad 115$

5.1 Introduction . . . . . . . . . . . . . . . . . . . . . . 117

5.2 Methods . . . . . . . . . . . . . . . . . . . . . . . . . . 118

5.2 .1 Animals . . . . . . . . . . . . . . . . . . . . . 118

5.2.2 Antibody production and labeling . . . . . . . . . . . . . 119

5.2 .3 Imaging . . . . . . . . . . . . . . . . . . . . . . . . . . . . . 119

5.2 .4 Biodistribution . . . . . . . . . . . . . . . . . . . . . . . 119

5.2 .5 Immunostaining and microscopy . . . . . . . . . . . 120

5.2 .6 Statistical Analyzes . . . . . . . . . . . . . . . . . . . 120

5.3 Results . . . . . . . . . . . . . . . . . . . . . . . . . . . . . 121

5.3.1 Soluble $A \beta$ does not increase in brain over time. . . . . . . . 122

5.3.2 Only ${ }^{89} \mathrm{Zr}$-bapineuzumab allows for in vivo diagnosis. . . . . . . 122

5.3.3 Biodistribution shows age-dependent difference in distribution . 123

5.3.4 ${ }^{89} \mathrm{Zr}$-solanezumab shows increased uptake in adipose tissue . . 124 
5.4 Discussion . . . . . . . . . . . . . . . . . . . . 127

References. . . . . . . . . . . . . . . . . . . . . 129

6 Discussion 133

6.1 Summary of key findings . . . . . . . . . . . . . . . . . . . . . . . . . . . . . . . . . . . . . . . .

6.2 Limitations. . . . . . . . . . . . . . . . . . . . . . . 136

6.3 Perspectives............................ 137

References. . . . . . . . . . . . . . . . . . 138

7 Valorisation 141

7.1 Therapeutic opportunities. . . . . . . . . . . . . . . . . . . . . . . . . 142

7.2 Diagnostic opportunities . . . . . . . . . . . . . . . 143

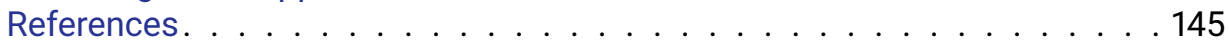

8 Summary 147

9 Samenvatting $\quad 151$

10 Curriculum Vitae $\quad 155$

11 List of publications $\quad 157$

12 Acknowledgements $\quad 161$ 



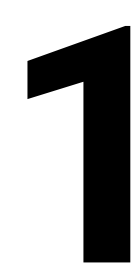

INTRODUCTION 


\subsection{Clinical and pathological feAtures of AlZheimer's DISEASE}

Alzheimer's disease (AD) is a major neurocognitive disorder that accounts for $60-80 \%$ of all dementia cases. $\mathrm{AD}$ is a progressive disease affecting one-third of the people over 85 years [1]. In mild stages people have significant impairments in memory and learning abilities, but in general they are still able to live on their own. As the disease progresses to a moderate stage, people need more and more supervision and personality changes start to occur. Finally, in the severe stage, people lose language abilities and need help with basic motor functions such as swallowing. The majority of $\mathrm{AD}$ patients die from aspiration pneumonia caused by swallowing problems [2].

Recently, new classification and criteria have been devised based on disease-specific biomarkers present in cerebrospinal fluid (CSF), positron-emission tomography (PET) and magnetic resonance imaging (MRI) [3] (see Table 1.1). A distinction is now made between prodromal $\mathrm{AD}$, where mild cognitive deficits and $\mathrm{AD}$ specific biomarkers are present, and mild cognitive impairment (MCI), where these same deficits are present, but AD specific biomarkers are absent [3].

\begin{tabular}{|c|c|c|c|c|}
\hline & $\begin{array}{c}\mathrm{AD} \\
\text { Diagnosis }\end{array}$ & $\begin{array}{l}\text { Presence of } \\
\text { impairment } \\
\text { of tests }\end{array}$ & $\begin{array}{l}\text { Evidence of } \\
\text { biomarkers } \\
\text { in vivo }\end{array}$ & $\begin{array}{l}\text { Additional } \\
\text { requirements }\end{array}$ \\
\hline Prodromal AD & Yes & Required & Required & $\begin{array}{c}\text { Absence of } \\
\text { dementia }\end{array}$ \\
\hline AD Dementia & Yes & Required & Required & $\begin{array}{c}\text { Presence of } \\
\text { dementia }\end{array}$ \\
\hline $\begin{array}{r}\text { Asymptomatic } \\
\text { at risk }\end{array}$ & No & Not present & Required & $\begin{array}{c}\text { Absence of } \\
\text { AD symptoms }\end{array}$ \\
\hline Presymptomatic & No & Not present & Not required & $\begin{array}{l}\text { Absence of AD } \\
\text { symptoms; } \\
\text { presence of } \\
\text { AD mutation }\end{array}$ \\
\hline $\begin{array}{l}\text { Mild Cognitive } \\
\text { Impairment }\end{array}$ & No & Not required & Not required & $\begin{array}{c}\text { Absence of } \\
\text { AD symptoms } \\
\text { or biomarkers }\end{array}$ \\
\hline
\end{tabular}

Table 1.1: New criteria for the diagnosis of AD (modified from [3]). Although cognitive tests and biomarkers are not specifically defined, they usually include a test such as the ADASCog. Biomarkers could be $\mathrm{A} \beta_{42}$ or tau levels in CSF or amyloid PET scan.

A distinction is also made between asymptomatic at-risk patients, who have positive biomarkers but no cognitive impairments, and pre-symptomatic patients, where people have autosomal dominant mutations that will (most likely) result in $\mathrm{AD}$, but do not show any symptoms yet. Especially from a therapeutic point of view, these groups might be the easier to treat, since they can receive preventive treatment.

Diagnosis and classification can also be done based on neuropathological hallmarks 
[4]. Hallmarks of AD consist of extracellular plaques mainly consisting out of amyloid $\beta$ $(\mathrm{A} \beta$ ) and intraneuronal neurofibrillary tangles (NFT) composed of hyperphosphorylated tau. This classification can only be done post-mortem, and until recently, this was the only method to have a certain diagnosis of AD. Other common causes of dementia are vascular dementia, dementia with Lewy bodies, and fronto-temporal lobe dementia [5].

\subsection{PATHOLOGY AND TREATMENT OPTIONS}

$\mathrm{AD}$ has a complicated etiology with several identified risk factors, but the exact cause remains unknown for the majority $(90 \%)$ of $\mathrm{AD}$ patients (sporadic $\mathrm{AD}, \mathrm{SAD}$ ). In a minority of cases, the cause can be reduced to mutations in a gene in the amyloid precursor protein (APP) processing pathway resulting in familial AD (FAD) [6]. To date, a number of mutations are known in the APP gene itself, or in presenilin-1 (PSEN1) or presnilin-2 (PSEN2) that form part of the gamma-secretase complex, responsible for the cleavage of APP to A $\beta$. These mutations result in a total increase of $A \beta$ production, or in a shift from $A \beta_{40}$ to the more aggregation-prone $A \beta_{42}$ (see Chapter 2, Figure 1).

Besides mutations in APP, PSEN1 or PSEN2, which will result in certain development of $\mathrm{AD}$, there are a large number of factors that increase the risk to develop $\mathrm{AD}$. The most important risk factor is age, although it is not clear how this factor contributes to the disease process [7]. It has been shown that amyloid depositions increase with age even in healthy persons without $\mathrm{AD}[8]$. Additionally, old age results in a state of chronic lowgrade inflammation, which might also influence the disease development [9].

Family history suggests that there is a hereditary component to $\mathrm{AD}$, and in recent years, a large number of genes have been implicated in AD etiology [10]. Most of these genes play a role in lipid metabolism, the proteasome system or inflammatory processes. The biggest genetic risk factor for $\mathrm{AD}$ is the APOE gene, which is involved in cholesterol metabolism. Homozygous carriers of the APOE $\varepsilon 4$ allele, run almost 15 times more risk to develop $\mathrm{AD}$ [11]. The APOE $\varepsilon 2$ allele on the other hand, confers a protective effect to $\mathrm{AD}$. While the exact role of APOE in the AD process is not completely known, APOE4 has been shown to lead to an impairment in $A \beta$ clearance and possible increased aggregation [12].

Apart from age, there are also a number of other non-genetic risk factors associated with $\mathrm{AD}$. One important factor associated with $\mathrm{AD}$ is a history of head trauma or traumatic brain injury (TBI) [13]. A $\beta$ has been shown to be increased after TBI in the short and long term [14, 15]. Additionally, head trauma also results in chronic inflammation, further exacerbating the risk for $\mathrm{AD}$ [16].

Vascular factors have also been associated with $\mathrm{AD}$ [17]. Hypertension, diabetes, smoking and heart disease have all been shown to play a role. The role of these factors is further complicated by the fact that they are also related to old age. In addition, it is not clear if these associations arose because of misdiagnosis of $\mathrm{AD}$ with vascular dementia [17]. Finally, lifestyle factors also influence AD risk. A Mediterranean-style diet and increased physical activity have been shown to reduce AD risk [18].

Several theories have been offered as to how AD starts and progresses to dementia [19]. At the moment, the leading hypothesis is the amyloid cascade hypothesis. This hypothesis states that an increase in $A \beta$ production or a decrease in $A \beta$ clearance leads to range of downstream processes eventually leading to neuronal death [20] (see Figure 
1). It has been shown that initially $A \beta$ will increase neuroinflammation as characterized by increased pro-inflammatory cytokines and gliosis [20]. Neuroinflammation and amyloid aggregation will cause mitochondrial damage and production of reactive oxygen species (ROS), resulting in a vicious circle where inflammation is causing further damage and ROS production causing more $A \beta$ production (see Figure 1) [21]. $A \beta$ and ROS subsequently cause hyperphosphorylation of tau, eventually leading to the formation of NFTs [22]. At this point, neurons start dying and the first clinical symptoms occur. The cholinergic system is affected first, with neuronal degeneration starting in the locus coeruleus, and causing memory loss [23]. NFT load also correlates better with clinical symptoms than $A \beta$ plaque load [24]. At later stages also the other monoamine systems, such as the glutamatergic system is affected, resulting in further cognitive complications. 
Mutations in PS1/PS2/APP,

family history, head trauma

lifestyl, vascular disease,...

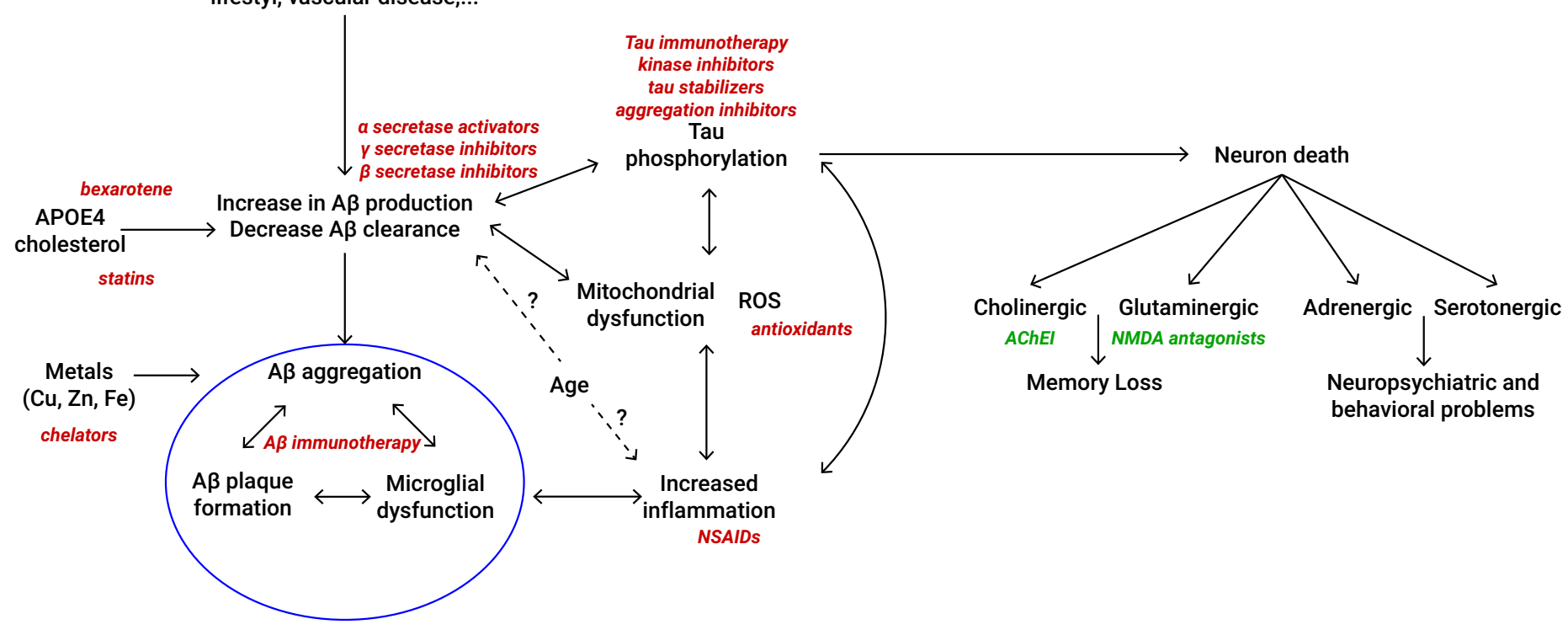

Figure 1.1: An increase in A $\beta$ production (caused by risk factors such as head trauma), or a decrease in clearance (caused by eg. APOE4) lead to A $\beta$ aggregation. This process can be accelerated under the influence of metals. Once $A \beta$ aggregates, a complex process of several interacting factors leads eventually to plaque formation, microglial dysfunction and increased inflammation. This inflammatory status will eventually lead to mitochondrial dysfunction and increased ROS production. All these factors interact to increase tau phosphorylation, leading to neuronal death and dysfunction of several neuronal neurotransmitter systems. In green are drugs counteracting $\mathrm{AD}$ pathology currently on the market. In red are drugs targeting various components of these pathway currently in clinical trials. The focus of this thesis ( $\mathrm{A} \beta$ immunotherapy) is emphasized in blue. 
At the moment, no disease modifying therapies are available. Currently available symptomatic therapies include acetylcholine esterase inhibitors which are effective in mild $\mathrm{AD}$, and memantine, a NMDA-antagonist, which can be used for treatment of moderate $\mathrm{AD}$ [25]. These drugs compensate the neuronal loss caused by AD (see Figure 1). However, they do not modify the processes leading up to this neuronal loss, and when this loss becomes too severe, these drugs become ineffective.

A large number of potential therapeutic drugs are currently being tested in clinical trials. They target various mechanisms involved in the disease process [26]. Whereas the large majority are designed to decrease amyloid production or aggregation, others are aimed at preventing tau phosphorylation or aggregation. To date all clinical trials conducted have yielded negative results (see Figure 1) [27].

\subsection{DIAGNOSIS OF AD}

Up until recently, diagnosis of AD was made post-mortem using immunohistochemical analysis of brain tissue sections. While post-mortem pathological evaluation is still considered the gold standard, new diagnostic tools have become available for in vivo diagnosis of $\mathrm{AD}$. Before the diagnosis of $\mathrm{AD}$ is made, cognitive performance is usually evaluated by means of one or more neuropsychological tests.

A short screening test, such as the mini mental state exam (MMSE) can be performed for fast initial assessment [28]. However, a longer cognitive assessment test specifically developed for assessing dementia is preferred. Two commonly used tests are the Alzheimer's Disease Assessment Scale - Cognitive section (ADASCog) and the Clinical Dementia Rating (CDR), which are also often used as inclusion criteria or endpoints in clinical trials [28]. One issue associated with these tests, is that they suffer from ceiling effects and are less sensitive for accurate diagnosis in early disease stages [29]. For this reason, effort is currently being invested in improving these scales.

Advances in technology and understanding of the disease have resulted in the development of novel biomarkers. At the moment, most biomarker-based diagnostics can be divided into two groups: CSF-based and imaging based. A large research effort has been spent in investigating and validating a large number of possible CSF biomarkers for $\mathrm{AD}$ [30]. However, in practice the most commonly used and best validated measurements are $A \beta_{1-42}$, total tau (t-tau) and phosphorylated tau ( $p$-tau), or combinations and ratios of these measurements [31]. These three measurements have now also been adopted into a consensus by the Alzheimer's biomarkers standardization initiative [32]. CSF $A \beta_{1-42}$ shows a negative correlation with disease progression, presumably because it is deposited into plaques, while tau shows a positive correlation with $\mathrm{AD}$ progression. These measures provide a high sensitivity ( $92 \%)$ and specificity $(\sim 82 \%)$ [33]. Additionally, they are also useful in predicting which MCI patients will progress to full-blown AD [34]. One concern about CSF values is the high intra- and inter-laboratory differences between the tests, which can result in up to $26 \%$ of individuals receiving a wrong diagnosis [35]. It is hoped that standardization of these tests should solve this problem [31].

Besides CSF biomarkers, imaging based markers have found widespread use in AD diagnosis. For imaging based diagnosis either structural MRI or PET based diagnostics are used. A structural MRI is most commonly used as a biomarker for diagnostic 
purposes [36]. A MRI scan can reveal medial temporal lobe atrophy, which can be used as a diagnostic marker for mild $\mathrm{AD}$ [37]. Visual rating scales of hippocampal atrophy provide a sensitivity and specificity of about $80-85 \%$, however, other measures are still needed to exclude other forms of dementia, since MRI measures alone do not provide enough specificity, as e.g. in the case of frontotemporal lobe dementia [38]. As a consequence, other MRI based measurements such as voxel based approaches to increase sensitivity and specificity are under investigation [39].

Another imaging approach is to use PET tracers to detect AD specific alterations such as reduced brain metabolism, amyloid deposits or tau tangles. Fluorodeoxyglucose-PET (FDG-PET) is the most commonly used and best established PET tracer for AD diagnosis [40]. FDG-PET can be used to measure glucose metabolism in the brain. In $\mathrm{AD}$ patients, glucose metabolism decreases over time, and this assessed on an FDG-PET scan. While FDG-PET shows a good sensitivity $(\sim 90 \%)$ to detect AD pathology, the specificity to differentiate from other dementias is lower ( 70\%) [40]. FDG-PET correlates well with functional assessments such as the ADAS-Cog and can be used to be predict future cognitive decline [41].

Recent advances in the development of amyloid binding PET tracers have enabled in vivo imaging of plaques. The first amyloid specific PET tracer, ${ }^{11} \mathrm{C}$ Pittsburgh compound $\mathrm{B}\left({ }^{11} \mathrm{C}-\mathrm{PiB}\right)$, was tested for the first time more than 10 years ago [42]. ${ }^{11} \mathrm{C}-\mathrm{PiB}$ is a thioflavin based analogue which binds to fibrillar amyloid deposits. In $\mathrm{AD}$ patients ${ }^{11} \mathrm{C}-\mathrm{PiB}$ retention is mainly observed in the cortex and is consistent with post-mortem studies. In addition, it has a good sensitivity ( 90\%) and specificity $(\sim 83 \%)$ [43]. The main drawback of ${ }^{11} \mathrm{C}-\mathrm{PiB}$ is the short half-life of only 20 minutes, limiting its use to PET centers that have access to an onsite cyclotron. Because of this problem, several ${ }^{18} \mathrm{~F}$-based tracers have been developed.

${ }^{18} \mathrm{~F}$-based tracers offer the advantage of a longer half-life (110 min) and this allows their routine use in all PET centers. The ${ }^{18} \mathrm{~F}$-based tracers are all similar to ${ }^{11} \mathrm{C}$-PiB and thioflavin in that they all bind to fibrillary amyloid. At the moment, the tracers florbetapir, flutemetamol and florbetaben have all gained FDA approval and are in routine clinical use [44]. A comparison of the different tracers is presented in Table 1.2.

\begin{tabular}{|c|c|c|c|c|}
\hline Name & Trade Name & Manufacturer & Sensitivity & Specificity \\
\hline${ }^{11} \mathrm{C}-\mathrm{PiB}$ & - & $\begin{array}{l}\text { University of } \\
\text { Pittsburgh }\end{array}$ & $\tilde{9} 0 \%$ & $\tilde{8} 3 \%$ \\
\hline Florbetapir & Amyvid & Eli Lilly & $\sim 87 \%$ & 9̃5\% \\
\hline Florbetaben & Neuraceq & Piramal & $\tilde{9} 7 \%$ & $\tilde{7} 8 \%$ \\
\hline Flutametamol & Vizamyl & GE Healthcar & $\tilde{9} 0 \%$ & $\tilde{8} 6 \%$ \\
\hline
\end{tabular}

Table 1.2: Comparison of the different commonly used amyloid PET tracers available (modified from [40])

Recently, also a PET ligand for tau has been developed. Although still in an early stage, this tracer is expected to provide a great deal of new information about AD progression and pathogenesis. ${ }^{18} \mathrm{~F}-\mathrm{AV}-1451$ binds specifically to paired helical filaments (PHF) [45] and first reports suggest that this tracer allows in vivo imaging of tau in patients [46]. 
One important advance of all these diagnostic techniques is that they have greatly improved our understanding about $\mathrm{AD}$ pathology. It is now clear that $\mathrm{A} \beta$ deposition start already a long time before the onset of the disease, even up to 10-15 years before the first clinical symptoms are present [47]. This highlights the fact that possible therapies will need to take into account that plaques are already present when patients present themselves with memory problems.

\subsection{AIMS AND OUTLINE}

The aim of this thesis was to investigate the use of antibodies for advanced diagnostic and therapeutic purposes.

Chapter 2 reviews the current literature about the role of inflammation and anti-A $\beta$ immunotherapy in $\mathrm{AD}$. The current leading $\mathrm{AD}$ hypothesis, the amyloid cascade hypothesis is discussed and recent evidence of other factors in $\mathrm{AD}$ are reviewed. Next, the importance of neuroinflammation, more specifically the role of microglia and the innate immune system is highlighted. Finally, we review effector mechanisms of $A \beta$ immunotherapy and provide a detailed overview of current clinical trials using immunotherapy.

In Chapter 3 an immunotherapy experiment is described that aims to bring further understanding in the role of effector mechanisms of $A \beta$ immunotherapy. To this end, $a$ recombinant mouse-human chimeric antibody (3D6) was produced in vitro. The human variant of this antibody (bapineuzumab) was also used in clinical trials, but with disappointing results. Two different isotypes of this antibody were produced: a mouse IgG1 (mIgG1) with anti-inflammatory properties, and a mouse IgG2a (mIgG2a) with inflammatory properties. Next, these antibodies were administered in both a preventive fashion (before onset of behavioral deficits) and therapeutic fashion (after onset of behavioral deficit) in a mouse model of AD.

As a model, we choose for the 5xFAD mouse which is transgenic for both human APP and PSEN1, with 3 familial AD mutations present in APP and 2 mutations present in PSEN1 [48]. While no tau tangles are present in this model, it shows a very fast onset of AD-like symptoms, with the first plaques appearing at 2-3 months of age. At 5 months of age, the first cognitive deficits are present. The 5xFAD model is one of the few mouse models which shows neuronal loss. Next, we performed behavioral testing of these animals to evaluate the effects of immunotherapy. On a molecular level, $A \beta$ load was evaluated and effects on gliosis were studied through immunofluorescence.

In Chapter 4, the focus is shifted to testing the use of an $A \beta$ antibody for diagnostic purposes. To this end, a human anti-A $\beta$ antibody (bapineuzumab) was labeled with a long-lived positron emitter $\left({ }^{89} \mathrm{Zr}\right)$ and its performance as an in vivo PET tracer was evaluated in the 5xFAD mouse model at various ages as a proof-of-concept. An isotype control and blocking experiment were also performed in the same animal model. After in vivo PET scans, bio-distribution studies were performed on macro-dissected brain and organs. Results were further supported by the use of autoradiography and immunofluorescence and histochemistry to prove specific binding of administered antibody in the brain.

Having established the technique of immuno-PET for amyloid imaging, we next compared the in vivo binding of different $A \beta$ antibodies in Chapter 5. We used three 
different antibodies targeting monomeric A $\beta$ (solanezumab), oligomers (20C2) and plaques (bapineuzumab). Antibodies were labeled again with ${ }^{89} \mathrm{Zr}$ and administered to $5 \mathrm{xFAD}$ animals at different ages. In vivo PET scans were performed and afterwards bio-distribution was performed. Immunohistochemistry and fluorescence was also performed to gain more insight in the different behavior of the antibodies.

Finally, we provide a summary of the key results and conclusions in Chapter 6. In addition, we also discuss the main limitations of the studies performed and offer some insights about future developments. 


\section{REFERENCES}

[1] Association A. 2015 Alzheimer's disease facts and figures [Journal Article]. Alzheimer's \& dementia: the journal of the Alzheimer's Association. 2015;11(3):332.

[2] Förstl H, Kurz A. Clinical features of Alzheimer's disease [Journal Article]. European archives of psychiatry and clinical neuroscience. 1999;249(6):288-290.

[3] Dubois B, Feldman HH, Jacova C, Cummings JL, DeKosky ST, Barberger-Gateau P, et al. Revising the definition of Alzheimer's disease: a new lexicon [Journal Article]. The Lancet Neurology. 2010;9(11):1118-1127. Available from: http: //www.sciencedirect.com/science/article/pii/S1474442210702234.

[4] Braak H, Braak E. Neuropathological stageing of Alzheimer-related changes [Journal Article]. Acta neuropathologica. 1991;82(4):239-259.

[5] Karantzoulis S, Galvin JE. Distinguishing Alzheimer's disease from other major forms of dementia [Journal Article]. Expert review of neurotherapeutics. 2014;

[6] Reitz C, Brayne C, Mayeux R. Epidemiology of Alzheimer disease [Journal Article]. Nature Reviews Neurology. 2011;7(3):137-152.

[7] Mattson MP. Pathways towards and away from Alzheimer's disease [Journal Article]. Nature. 2004;430(7000):631-639. Available from: http://dx.doi .org/10.1038/ nature02621.

[8] Yamada M, Tsukagoshi H, Otomo E, Hayakawa M. Systemic amyloid deposition in old age and dementia of Alzheimer type: the relationship of brain amyloid to other amyloid [Journal Article]. Acta neuropathologica. 1988;77(2):136-141.

[9] Chung HY, Cesari M, Anton S, Marzetti E, Giovannini S, Seo AY, et al. Molecular inflammation: Underpinnings of aging and age-related diseases [Journal Article]. Ageing Research Reviews. 2009;8(1):18-30. Available from: http://www. sciencedirect.com/science/article/pii/S1568163708000299.

[10] Bettens K, Sleegers K, Van Broeckhoven C. Genetic insights in Alzheimer's disease [Journal Article]. The Lancet Neurology. 2013;12(1):92-104. Available from: http: //www.sciencedirect.com/science/article/pii/S1474442212702594.

[11] Laws SM, Hone E, Gandy S, Martins RN. Expanding the association between the APOE gene and the risk of Alzheimer's disease: possible roles for APOE promoter polymorphisms and alterations in APOE transcription [Journal Article]. Journal of neurochemistry. 2003;84(6):1215-1236.

[12] Liu CC, Kanekiyo T, Xu H, Bu G. Apolipoprotein E and Alzheimer disease: risk, mechanisms, and therapy [Journal Article]. Nature reviews Neurology. 2013;9(2):106-118. Available from: http://www.ncbi.nlm.nih.gov/pmc/ articles/PMC3726719/. 
[13] Mortimer JA, Van Duijn CM, Chandra V, Fratiglioni L, Graves AB, Heyman A, et al. Head Trauma as a Risk Factor for Alzheimer's Disease: A Collaborative Re-Analysis of Case-Control Studies [Journal Article]. International Journal of Epidemiology. 1991;20(Supplement 2):S28-S35. Available from: http://ije.oxfordjournals. org/content/20/Supplement_2/S28.abstract.

[14] Marklund N, Farrokhnia N, Hånell A, Vanmechelen E, Enblad P, Zetterberg H, et al. Monitoring of $\beta$-amyloid dynamics after human traumatic brain injury [Journal Article]. Journal of neurotrauma. 2014;31(1):42-55.

[15] Mielke MM, Savica R, Wiste HJ, Weigand SD, Vemuri P, Knopman DS, et al. Head trauma and in vivo measures of amyloid and neurodegeneration in a populationbased study [Journal Article]. Neurology. 2014;82(1):70-76.

[16] Ramlackhansingh AF, Brooks DJ, Greenwood RJ, Bose SK, Turkheimer FE, Kinnunen $\mathrm{KM}$, et al. Inflammation after trauma: Microglial activation and traumatic brain injury [Journal Article]. Annals of Neurology. 2011;70(3):374-383. Available from: http://dx.doi.org/10.1002/ana.22455.

[17] van der Flier WM, Scheltens P. Epidemiology and risk factors of dementia [Journal Article]. Journal of Neurology, Neurosurgery \& Psychiatry. 2005;76(suppl 5):v2-v7. Available from: http://jnnp.bmj . com/content/76/suppl_5/v2.short.

[18] Scarmeas N, Luchsinger JA, Schupf N, Brickman AM, Cosentino S, Tang MX, et al. Physical activity, diet, and risk of Alzheimer disease [Journal Article]. Jama. 2009;302(6):627-637.

[19] Maccioni RB, Perry G. Current hypotheses and research milestones in Alzheimer's disease. Springer Science \& Business Media; 2009.

[20] Selkoe DJ, Hardy J. The amyloid hypothesis of Alzheimer's disease at 25 years [Journal Article]. EMBO Molecular Medicine. 2016;p. e201606210.

[21] Leuner K, Müller WE, Reichert AS. From mitochondrial dysfunction to amyloid beta formation: novel insights into the pathogenesis of Alzheimer's disease [Journal Article]. Molecular neurobiology. 2012;46(1):186-193.

[22] Ballatore C, Lee VMY, Trojanowski JQ. Tau-mediated neurodegeneration in Alzheimer's disease and related disorders [Journal Article]. Nature Reviews Neuroscience. 2007;8(9):663-672.

[23] Mesulam M. The cholinergic lesion of Alzheimer's disease: pivotal factor or side show? [Journal Article]. Learning \& Memory. 2004;11(1):43-49.

[24] Jack Jr CR, Knopman DS, Jagust WJ, Petersen RC, Weiner MW, Aisen PS, et al. Tracking pathophysiological processes in Alzheimer's disease: an updated hypothetical model of dynamic biomarkers [Journal Article]. The Lancet Neurology. 2013;12(2):207-216. Available from: http://www.sciencedirect. com/science/article/pii/S1474442212702910. 
[25] Yiannopoulou KG, Papageorgiou SG. Current and future treatments for Alzheimer's disease [Journal Article]. Therapeutic Advances in Neurological Disorders. 2013;6(1):19-33. Available from: http://tan. sagepub.com/content/6/1/19. abstract.

[26] Mangialasche F, Solomon A, Winblad B, Mecocci P, Kivipelto M. Alzheimer's disease: clinical trials and drug development [Journal Article]. The Lancet Neurology. 2010;9(7):702-716.

[27] Schneider LS, Mangialasche F, Andreasen N, Feldman H, Giacobini E, Jones R, et al. Clinical trials and late-stage drug development for Alzheimer's disease: an appraisal from 1984 to 2014 [Journal Article]. Journal of internal medicine. 2014;275(3):251-283. Available from: http://www.ncbi.nlm.nih.gov/pmc/ articles/PMC3956752/.

[28] Sheehan B. Assessment scales in dementia [Journal Article]. Therapeutic advances in neurological disorders. 2012;p. 1756285612455733.

[29] Raghavan N, Samtani MN, Farnum M, Yang E, Novak G, Grundman M, et al. The ADAS-Cog revisited: Novel composite scales based on ADAS-Cog to improve efficiency in MCI and early AD trials [Journal Article]. Alzheimer's \& Dementia. 2013;9(1, Supplement):S21-S31. Available from: http://www.sciencedirect. $\mathrm{com} / \mathrm{science/article/pii/S1552526012023850.}$

[30] Hu WT, Chen-Plotkin A, Arnold SE, Grossman M, Clark CM, Shaw LM, et al. Novel CSF biomarkers for Alzheimer's disease and mild cognitive impairment [Journal Article]. Acta Neuropathologica. 2010;119(6):669-678. Available from: http://dx . doi .org/10.1007/s00401-010-0667-0.

[31] Blennow K, Dubois B, Fagan AM, Lewczuk P, de Leon MJ, Hampel H. Clinical utility of cerebrospinal fluid biomarkers in the diagnosis of early Alzheimer's disease [Journal Article]. Alzheimer's \& Dementia. 2015;11(1):58-69. Available from: http : //www.sciencedirect.com/science/article/pii/S1552526014000661.

[32] Molinuevo JL, Blennow K, Dubois B, Engelborghs S, Lewczuk P, Perret-Liaudet A, et al. The clinical use of cerebrospinal fluid biomarker testing for Alzheimer's disease diagnosis: A consensus paper from the Alzheimer's Biomarkers Standardization Initiative [Journal Article]. Alzheimer's \& Dementia. 2014;10(6):808817. Available from: http://www.sciencedirect.com/science/article/pii/ S1552526014001095.

[33] Duits FH, Teunissen CE, Bouwman FH, Visser PJ, Mattsson N, Zetterberg H, et al. The cerebrospinal fluid "Alzheimer profile": Easily said, but what does it mean? [Journal Article]. Alzheimer's \& Dementia. 2014;10(6):713723.e2. Available from: http://www.sciencedirect.com/science/article/ pii/S1552526014000296.

[34] Vos SJ, van Rossum IA, Verhey F, Knol DL, Soininen H, Wahlund LO, et al. Prediction of Alzheimer disease in subjects with amnestic and nonamnestic MCI [Journal Article]. Neurology. 2013;80(12):1124-1132. 
[35] Vos SJB, Visser PJ, Verhey F, Aalten P, Knol D, Ramakers I, et al. Variability of CSF Alzheimer?s Disease Biomarkers: Implications for Clinical Practice [Journal Article]. PLoS ONE. 2014;9(6):e100784. Available from: http://dx.doi .org/10 . $1371 \% 2$ F journal pone. 0100784.

[36] Bocchetta M, Galluzzi S, Kehoe PG, Aguera E, Bernabei R, Bullock R, et al. The use of biomarkers for the etiologic diagnosis of MCI in Europe: An EADC survey [Journal Article]. Alzheimer's \& Dementia. 2015;11(2):195-206.e1. Available from: http: //www.sciencedirect.com/science/article/pii/S1552526014024650.

[37] Frisoni GB, Fox NC, Jack CR, Scheltens P, Thompson PM. The clinical use of structural MRI in Alzheimer disease [Journal Article]. Nature Reviews Neurology. 2010;6(2):67-77.

[38] Fennema-Notestine C, Hagler DJ, McEvoy LK, Fleisher AS, Wu EH, Karow DS, et al. Structural MRI biomarkers for preclinical and mild Alzheimer's disease [Journal Article]. Human Brain Mapping. 2009;30(10):3238-3253. Available from: http: //dx.doi.org/10.1002/hbm. 20744.

[39] Tondelli M, Wilcock GK, Nichelli P, De Jager CA, Jenkinson M, Zamboni G. Structural MRI changes detectable up to ten years before clinical Alzheimer's disease [Journal Article]. Neurobiology of Aging. 2012;33(4):825.e25-825.e36. Available from: http: //www.sciencedirect.com/science/article/pii/S0197458011002016.

[40] McConathy J, Sheline YI. Imaging Biomarkers Associated With Cognitive Decline: A Review [Journal Article]. Biological Psychiatry. 2015;77(8):685692. Available from: http://www. sciencedirect.com/science/article/pii/ s0006322314006787.

[41] Landau SM, Harvey D, Madison CM, Koeppe RA, Reiman EM, Foster NL, et al. Associations between cognitive, functional, and FDG-PET measures of decline in AD and MCI [Journal Article]. Neurobiology of Aging. 2011;32(7):12071218. Available from: http://www.sciencedirect.com/science/article/ pii/S0197458009002279.

[42] Klunk WE, Engler H, Nordberg A, Wang Y, Blomqvist G, Holt DP, et al. Imaging brain amyloid in Alzheimer's disease with Pittsburgh Compound-B [Journal Article]. Annals of Neurology. 2004;55(3):306-319. Available from: http://dx.doi.org/ 10.1002/ana. 20009.

[43] Cohen AD, Klunk WE. Early detection of Alzheimer's disease using PiB and FDG PET [Journal Article]. Neurobiology of Disease. 2014;72, Part A:117122. Available from: http://www. sciencedirect.com/science/article/pii/ S0969996114001107.

[44] Nordberg A, Rinne JO, Kadir A, Langstrom B. The use of PET in Alzheimer disease [Journal Article]. Nat Rev Neurol. 2010;6(2):78-87. Available from: http://dx . doi.org/10.1038/nrneurol.2009.217. 
[45] Xia CF, Arteaga J, Chen G, Gangadharmath U, Gomez LF, Kasi D, et al. [18 F] T807, a novel tau positron emission tomography imaging agent for Alzheimer's disease [Journal Article]. Alzheimer's \& Dementia. 2013;9(6):666-676.

[46] Schöll M, Lockhart SN, Schonhaut DR, O’Neil JP, Janabi M, Ossenkoppele R, et al. PET Imaging of Tau Deposition in the Aging Human Brain [Journal Article]. Neuron. 2016;89(5):971-982. Available from: http://www.sciencedirect.com/ science/article/pii/S0896627316000532.

[47] Jack CR, Knopman DS, Jagust WJ, Petersen RC, Weiner MW, Aisen PS, et al. Update on hypothetical model of Alzheimer's disease biomarkers [Journal Article]. Lancet neurology. 2013;12(2):207-216. Available from: http: //www.ncbi.nlm.nih.gov/ pmc/articles/PMC3622225/.

[48] Oakley H, Cole SL, Logan S, Maus E, Shao P, Craft J, et al. Intraneuronal $\beta$ Amyloid Aggregates, Neurodegeneration, and Neuron Loss in Transgenic Mice with Five Familial Alzheimer's Disease Mutations: Potential Factors in Amyloid Plaque Formation [Journal Article]. The Journal of Neuroscience. 2006;26(40):1012910140. Available from: http://www.jneurosci.org/content/26/40/10129. abstract. 


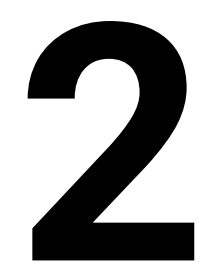

\section{INFLAMMATION AND ALZHEIMER'S DISEASE: LESSONS FROM IMMUNOTHERAPY}

Jo A.A. Stevens, Pilar Martinez-Martinez, Mario Losen Submitted 


\begin{abstract}
Alzheimer's disease (AD), the most common cause of dementia, poses an enormous health burden and at the moment no disease-modifying treatment is available. One of the hallmarks of the disease is the buildup of amyloid- $\beta$ (A $\beta$ ) plaques in the brain. In the last 20 years, over a 100 clinical trials using $A \beta$ immunotherapy have been conducted using approximately 15 different antibodies and 10 different $A \beta$ vaccinations involving over 25000 patients. In this review, we provide a current overview of the involvement of the immune system in $\mathrm{AD}$, with a special focus on the antibody effector mechanisms of amyloid immunotherapy, and lessons that can be learned. In addition, we provide an up-to-date overview of finished and ongoing trials using immunotherapy. While therapies have been unable to halt the disease so far, twelve different $A \beta$ immunotherapies are still being tested in patients, with some of them showing promising preliminary results.
\end{abstract}


Alzheimer's disease $(\mathrm{AD})$ is the most common cause of dementia worldwide and no cure is available at the moment. $\mathrm{AD}$ poses and will pose an enormous burden for society, it is expected that by 2050 over 130 million people will suffer from it [1]. The disease is characterized by the build-up of extracellular plaques composed of amyloid- $\beta$ $(\mathrm{A} \beta)$, and of intracellular tangles composed of hyperphosphorylated tau protein. At the moment, several clinical trials are ongoing or have been completed using immunotherapy against $A \beta$. $A \beta$ immunotherapy aims to alter $A \beta$ deposition by inducing a humoral immune response to $A \beta$ (active) or administering anti-A $\beta$ antibodies (passive). Here, we focus on the crucial role of the immune system in the neuropathophysiology of $\mathrm{AD}$, the relationship to $\mathrm{A} \beta$ immunotherapy and lessons learned from immunotherapy trials.

\subsection{THE AMYLOID CASCADE HYPOTHESIS}

About 1-5\% of $\mathrm{AD}$ patients suffer from early-onset familial $\mathrm{AD}$ (EOFAD), which is characterized by a mendelian inheritance pattern and an early-onset (before $<65$ ) of the disease [2]. EOFAD is caused by mutations in amyloid precursor protein (APP), presenilin-1 (PSEN1) or presenilin-2 (PSEN2), two proteins which are part of the $\gamma$-secretase complex, which is responsible for the generation of $A \beta$ [3]. Of these, mutation in PSEN1 are the most common [4]. However, the majority of AD patients suffer from sporadic $\mathrm{AD}$ (SAD), of which the cause is unknown, and which usually has a late onset $(>65)$. The heritability of SAD patients has been estimated to be between $60-80 \%$, so the environment also plays a significant role [5]. While clinically there are some differences between EOFAD and SAD in the onset of the disease, in that EOFAD usually presents with worse symptoms, histologically both groups have the same hallmarks, i.e. $\mathrm{A} \beta$ plaques and tau tangles.

The combined fact that patients suffering from EOFAD always have mutations in proteins involved in the APP processing pathway and that $A \beta$ plaques are always present in the brain of $\mathrm{AD}$ patients, led to the postulation of the amyloid cascade hypothesis.

Initially, the hypothesis suggested that $A \beta$ is toxic, and the buildup of $A \beta$ in the plaques gives rise to $\mathrm{AD}$ by causing neurons to stop functioning and eventually dying off [6].

To understand the amyloid cascade hypothesis, it is useful to take a closer look at the APP processing pathway. APP is a 110-135 kDa membrane bound protein, of which the exact physiological function is unknown [7]. Data from knock-out models and studies of the APP promoter suggest that APP plays a role in neuronal development, but the function in later life remains unclear [8]. APP can be cleaved by $\alpha$-secretase, of which ADAM10 is the most abundant in the brain [9]. Cleavage of APP by $\alpha$-secretase, results in two fragments called soluble $\operatorname{APP} \alpha(\operatorname{sAPP} \alpha)$ and the membrane bound C-Terminal Fragment- $\alpha(\mathrm{CTF} \alpha)$. Subsequently, CTF $\alpha$ can be cleaved by $\gamma$-secretase resulting in the p3 fragment and the cytosolic Amyloid Intracellular Cytoplasmic Domain (AICD) (see Figure 2.1). The p3 fragment is in fact a truncated form of A $\beta$, but is thought to be pathologically irrelevant [10]. Cleavage by $\alpha$-secretase puts APP to the so-called non-amyloidogenic arm, since no $A \beta$ is generated.

APP can also be cleaved by a $\beta$-secretase, of which BACE1 is the pathologically relevant secretase for $\mathrm{AD}$. Cleavage by BACE1 will result in an SAPP $\beta$ fragment and a 
CTF $\beta$ fragment. Subsequent cleavage of CTF $\beta$ by $\gamma$-secretase will eventually result in generation of $A \beta$ and the AICD fragment (see Figure 2.1) [11]. This is called the amyloidogenic pathway.

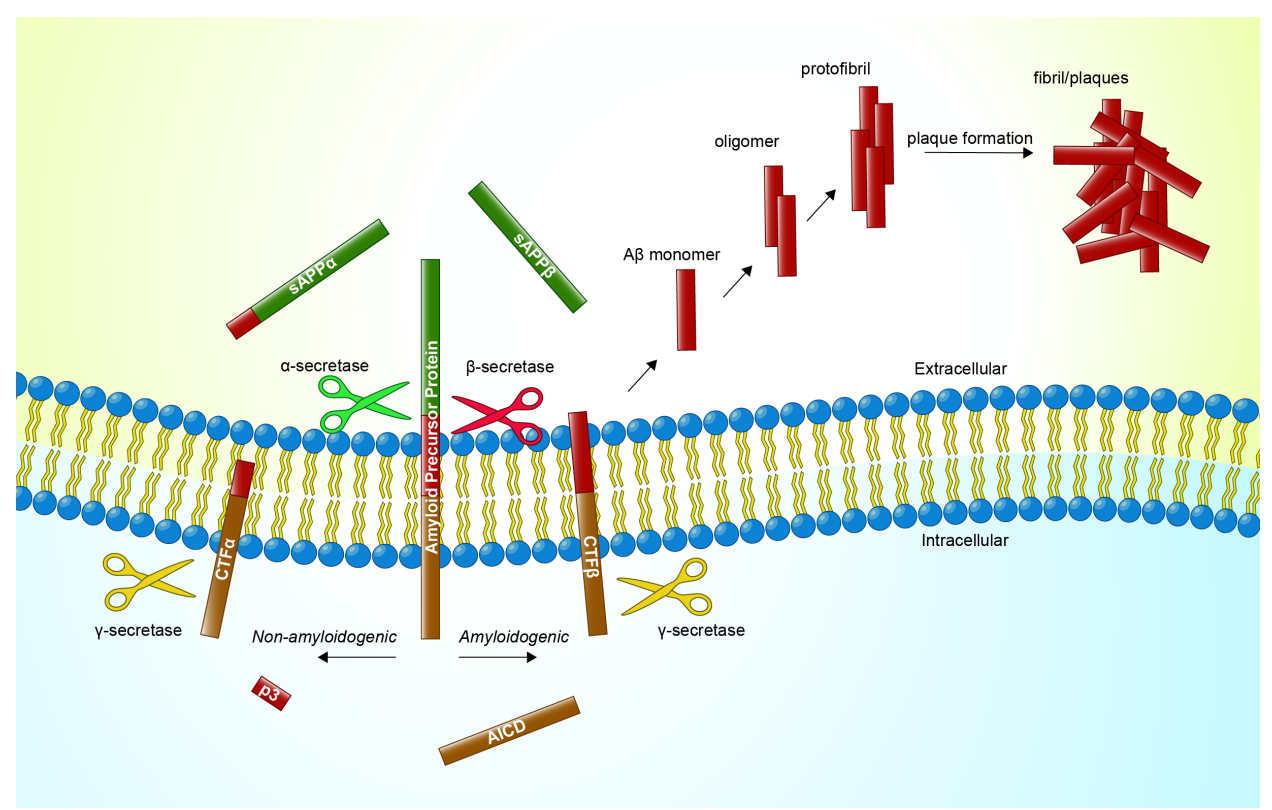

Figure 2.1: APP can be cleaved by $\alpha$-secretase resulting in the generation of sAPP $\alpha$ and CTF $\alpha$. This will shift APP processing to the non-amyloidogenic pathway, since subsequent processing by $\gamma$-secretase (yellow scissors) will result in the non-toxic p3 fragment and the AICD fragment. However, if APP is cleaved by $\beta$-secretase (red scissors), APP processing will shift to the amyloidogenic pathway, resulting in generation of A $\beta$ by subsequent processing of $\gamma$-secretase. Next, soluble $A \beta$ monomers can start to aggregate to oligomers and protofibrils. Finally, protofibrils will aggregate into non-soluble fibrils and be deposited into amyloid plaques.

Depending on the exact cleavage of $\gamma$-secretase, different sizes of $A \beta$ are formed which can range from 37-49 amino acids [11]. From these different proteins, $A \beta 40$ is viewed as the normal cleavage product, while $A \beta 42$ has been suggested to be the pathogenic form eventually resulting in $\mathrm{AD}$ [12]. Patient data also shows that they produce more A $\beta 42$ than healthy individuals [13]. Furthermore, A $\beta 42$ has been shown to have a higher tendency to aggregate [14] and mutations in APP, PSEN1 or PSEN2 result in generation of more $A \beta 42$, or in a shift in processing towards the amyloidogenic pathway [15].

This generation of more $A \beta 42$ resulting in plaques has long been considered the central event in the amyloid cascade hypothesis. In recent years however, the hypothesis has been refined due to conflicting evidence about A $\beta 42$ [16]. Monomeric A 442 aggregates into oligomers, protofibrils, fibrils and eventually gets deposited into plaques (see Figure 2.1). Contradictory data exists about which form is more toxic, but recent research suggests that it is the oligomeric form, and not the fibrillar form which is more toxic [17]. Additionally, oligomeric A $\beta$ in CSF has been shown to correlate better with clinical progression of $\mathrm{AD}$, while tau or $\mathrm{A} \beta 42$ levels did not [18]. Other research 
suggests that other forms of $A \beta$, such as $N$-truncated $A \beta$, might be the more toxic forms and the actual initiator of the disease [19].

\subsection{OTHER FACTORS IN AD}

$\mathrm{AD}$ is a multifactorial disease and there are different theories which may explain its etiology and progression. Additionally, the clinical picture is complicated due to the fact that $60 \%$ of $\mathrm{AD}$ patients have at least three comorbidities [20].

Besides $A \beta$, tau tangles are the most important hallmark of the disease brain. Tau is an intracellular, highly soluble protein whose main function is to stabilize axon microtubuli. Under physiological conditions it is mainly contained to the axonal compartment, while under pathological conditions it gets missorted to the somadentrical compartment. Not only in $\mathrm{AD}$, but also in other tauopathies, tau gets hyperphosphorylated, missorted, starts forming intracellular tangles, and eventually causes neuronal dysfunction [21]. Data from animal models suggests that there is at least a synergy between the two pathological hallmarks of the disease, tau and $A \beta$. Some studies show that tau pathology is worsened with the presence of $A \beta$ [22]. Other studies, where tau knockout mice are crossed with $\mathrm{AD}$ mouse models with $\mathrm{A} \beta$ plaques, show that without tau, $A \beta$ does not have toxic effects on the brain, or there is a reduced plaque burden $[23,24]$. Interestingly, cognitive decline seems to correlate better with tau pathology, suggesting that tau might be the driving force in clinical $\mathrm{AD}$ progression [25].

The strongest genetic risk factor for AD is the APOE $\varepsilon 4$ allele. APOE is the most common lipoprotein in the brain and consists of three isoforms. The $\varepsilon 4$ allele occurs in about $15 \%$ of the normal population, but is present more in the $\mathrm{AD}$ population with a percentage of $40 \%$. Homozygous carriers with $\varepsilon 4 / \varepsilon 4$ even run 15 times more risk to develop $\mathrm{AD}$ [26]. Carriers with the $\varepsilon 2$ allele on the other hand, seem to be protected for $\mathrm{AD}$. Despite the fact that APOE4 is such a strong risk factor, it is not yet clear whether the increased risk is caused by a toxic gain-of-function, a neuroprotective loss-of-function, or both [16]. APOE4 has been suggested to have an effect on A $\beta$ aggregation and clearance (for a review see: Liu et al [27]). Interestingly, the $\varepsilon 4$ allele also has significant effects on neuroinflammation. In $\varepsilon 3 / \varepsilon 4$ carriers, the innate immune response after LPS administration is stronger than in $\varepsilon 3 / \varepsilon 3$ carriers [28]. Furthermore, post-mortem analysis has shown increased microglial activation in the brain of APOE4 AD patients [29] (for a review: Tai et al [30]).

\subsection{THE IMMUNE SYSTEM AND AD}

Recently, the role of neuroinflammation in $\mathrm{AD}$ has become more important. Already in his original descriptions of the disease, Alois Alzheimer mentioned that plaques were surrounded by microglia, the macrophages of the brain, but it was believed for many years that the immune system played only a secondary role in the disease [31]. Other clues about the importance of the immune system in $\mathrm{AD}$ came from epidemiological studies showing that anti-inflammatory drugs might be protective against AD [32, 33]. However, clinical trials using non-steroid anti-inflammatory drugs (NSAIDs) failed to show any therapeutic effect $[34,35]$. 
While both the innate and the adaptive immune system play a role in $\mathrm{AD}$, current knowledge points to a much greater involvement of the innate immune system [36]. In the next sections, we will highlight more in depth the various components of the innate immune system that play a role in $\mathrm{AD}$, namely microglia, the complement system, and the inflammasome.

\subsubsection{THE ROLE OF MICROGLIA IN AD}

Microglia can detect danger and pathogen associated molecular patterns (DAMPs and PAMPs) through receptors. It has been known for many years that microglia can be activated by binding of fibrillary A $\beta$ to CD36 [37]. Upon binding of A $\beta$, CD36 will recruit Toll-like receptors and will form a complex with TLR4 and TLR6, resulting in activation of microglia and secretion of IL-1 $\beta$ [38]. This sustained pro-inflammatory response will eventually lead to a downregulation of critical $A \beta$ degrading enzymes, resulting in an impairment of microglial A $\beta$ clearance [39]. Additionally, it has been shown that tau can also activate microglia resulting in the release of IL-6 [40]. While there have been ample studies, the role of microglia activation in the disease process remains unclear [41]. Current evidence points to a protective role of the cells in early stages of AD, while prolonged activation might be detrimental [42].

As stated before, microglia can recognize $A \beta$ through means of CD36, after which phagocytosis can follow and subsequent degradation by the proteases neprilysin and insulin-degrading enzyme [43]. In AD patients, microglial clearance has been shown to be impaired [44], which is caused by the sustained pro-inflammatory environment of the AD brain [43].

In the last decade several findings have further established the important role of microglia in the disease. First, genome wide association studies (GWAS) revealed that a number of immune related genes are associated with sporadic AD, of which CD33 is an important gene related to microglia [45]. CD33 is a type I transmembrane protein which is predominantly expressed on microglia cells. In patients, CD33 expression is increased and it has been shown that CD33 can be activated by binding of sialic acid residues on glycolipids and proteins present in amyloid plaques leading to inhibition of microglial A $\beta$ internalization [46].

GWAS further brought the immune system to the forefront of AD research with the discovery that rare variant mutations in TREM2 increase the risk to develop AD with a factor similar to APOE4 [47], a finding which has been confirmed by a number of other studies [48]. TREM2 is a receptor of the innate immune system expressed on a variety of cells such as monocytes, macrophages and microglia. TREM2 is involved in microglia and macrophages activation, and can be stimulated by negatively charged lipids such as LPS and phospholipids [48]. Of these lipids, sphingomyelin has been shown to be one of the most potent activators [49]. TREM2 has also been shown to have an anti-inflammatory function, but its exact role in the pathogenesis of $\mathrm{AD}$ remains elusive [50]. Studies in AD mouse models and TREM2 knock-outs report conflicting results from beneficial to detrimental [51]. The exact cause for this discrepancy is not clear, but could be due to different mouse models used, different ages of the mice, or the different type of knockout (knockout of the full-protein or knockout of the extracellular part of TREM2). 


\subsubsection{COMPLEMENT AND THE INFLAMMASOME IN AD}

The complement system plays an important in $\mathrm{AD}$ progression. Already many years ago, it was shown that $A \beta$ can directly activate the classical complement pathway by binding to $\mathrm{Clq}$ [52]. Activation of C1q occurs through binding of $\mathrm{A} \beta$ to the collagen-like domain of C1q [53]. However, in mice models of $\mathrm{AD}$, it has been shown that complement activation has been weaker compared to human complement activation, which might be due to the different structure of C1q in mice [54, 55].

Interestingly, the ceramide transporter (CERT), which is abundant in brain and the only human protein known to transfer ceramide between membranes, can also activate $\mathrm{Clq}$ via the classical pathway and is associated with plaques [56]. Ceramide is the backbone of all complex sphingolipids which play both a structural role in membranes as well as act as second messengers balancing death and survival signals in the cell [57]. This provides an additional link between lipid metabolism and AD [58, 59].

Perhaps counterintuitively, complement can also have beneficial and protective effects in the CNS. This is evidenced by the fact that Clq is also responsible for synaptic pruning, which plays an important role during development [60]. C1q has also been shown to have neuroprotective effects, but only in the absence of other downstream complement factors [61]. In $\mathrm{AD}$, the role of complement seems to be more complicated. Deficiency of $\mathrm{Clq}$ has been shown to ameliorate neuropathology in an AD mouse model, which is probably due to a reduction in inflammation [62]. A comparative genome-wide expression analysis in different $\mathrm{AD}$ mouse models, also pinpointed $\mathrm{Clq}$ as a central hub in both $A \beta$ and tau mouse models, further implicating complement in AD pathogenesis [63].

In healthy wild type animals, C3 deficiency seems to be protective for age-related memory loss, which is in line with Clq studies [64]. Additionally, recent results also point out that C3 by itself impair phagocytic abilities of microglia, further suggesting that $\mathrm{C} 3$ plays a detrimental role in $\mathrm{AD}$ [65]. Two studies have shown that $\mathrm{C} 3$ might have a beneficial role in ameliorating $\mathrm{AD}$ by increasing phagocytosis [66, 67]. These studies are limited however in that they make use of C3 knock-out models, and therefore do not have prolonged activation and deposition of $\mathrm{C} 3$ as observed in patients or other mouse models [68].

The importance of complement also became clear with recent GWAS results showing that Complement Receptor 1 (CR1) is a potential risk factor for AD [69]. CR1 acts as an inhibitor of the complement pathway by binding and subsequent clearing of C3b or C4b, two factors of the complement cascade [70]. So far, studies investigating the described mutation in CR1 have been unable to pinpoint its exact functional contribution to $\mathrm{AD}$ [71]. A possible reason for this is that mice do not express CR1 on erythrocytes, while it has been shown that $A \beta 42$ can be cleared by binding of C3-opsonized A $\beta$ to CR1 on human erythrocytes [72].

The inflammasome is a protein complex where several signaling pathways from the innate immune system, such as those initiated by TLRs, converge and result in the production of cytokines by activation of caspase-1 [73]. Several inflammasome complexes have been discovered and in $\mathrm{AD}$ Nod-like receptor pyrin domain inflammasomes (NLRP) have been implicated in the disease process. In particular, NLRP3, which is a cytosolic inflammasome sensor, has been shown to be activated by 
$A \beta$ [74]. The activation of the NLRP3 inflammasome results in the production of IL-1 $\beta$, which has been reported to be elevated in AD patients [75], just as the levels of NLRP3 inflammasome itself [76]. Finally, studies with AD mouse models have shown that disabling the NLRP3 inflammasome pathway, restores memory and improves A $\beta$ clearance [77].

\subsection{EFFECTOR MECHANISMS OF A $\beta$ IMMUNOTHERAPY}

Two different approaches of anti-A $\beta$ immunotherapy can be employed: active or passive immunization. In active immunization, partial or full-length $A \beta$ is injected into the patient together with an adjuvant (vaccination). The patient will produce anti-A $\beta$ antibodies which will clear-away the plaques. Classes of adjuvants are (1) mineral compounds such as alum, (2) bacterial products such as lipopolysaccharide (LPS), (3) emulsion-based adjuvants such as Freunds, and (4) immunestimulatory compounds such as cytokines [78]. Adjuvants are necessary to, amongst other things, enable a more rapid immune response, increase antibody titers or overcome immune senescence which is common in elderly patients [79]. They exert their action by acting like a depot that releases antigen over a prolonged period of time (eg. emulsion based adjuvants), or by stimulating the immune system by increasing cytokine production, recruit immune cells, or activation of inflammasomes (eg. alum) [80].

In passive immunization, (recombinant) antibodies are administered to the patient for as long as necessary, in other words the patient does not need to make its own anti- $\mathrm{A} \beta$ antibodies. While passive immunization offers the benefit that the therapy can be more controlled (e.g. immune response) and the target can be better chosen, it also has significant cost disadvantages, since antibody production in these quantitates is costly and time consuming. Additionally, there is the risk that patients will develop neutralizing antibodies, which might also lead to serious consequences such as anaphylactic shock [81]. With active immunization on the other hand, there is the risk that the patients might not generate an antibody titer at all, because of self-tolerance against $\mathrm{A} \beta$ or because of immune system senescence at old age [82].

While some effector mechanisms of immunotherapy have been studied on post-mortem brain tissue of patients, most of the details have been elucidated by immunotherapy studies in preclinical mouse models. Three possible mechanisms have been proposed: (1) Fc-Receptor mediated phagocytosis; (2) peripheral sink action; and (3) catalytic dissolution (see Figure 2.2). The three mechanisms are not mutually exclusive and may act in concert. Surprisingly, a possible role of the complement system has not been investigated in immunotherapy. We will discuss all three mechanisms in more detail, and review the evidence for them. 


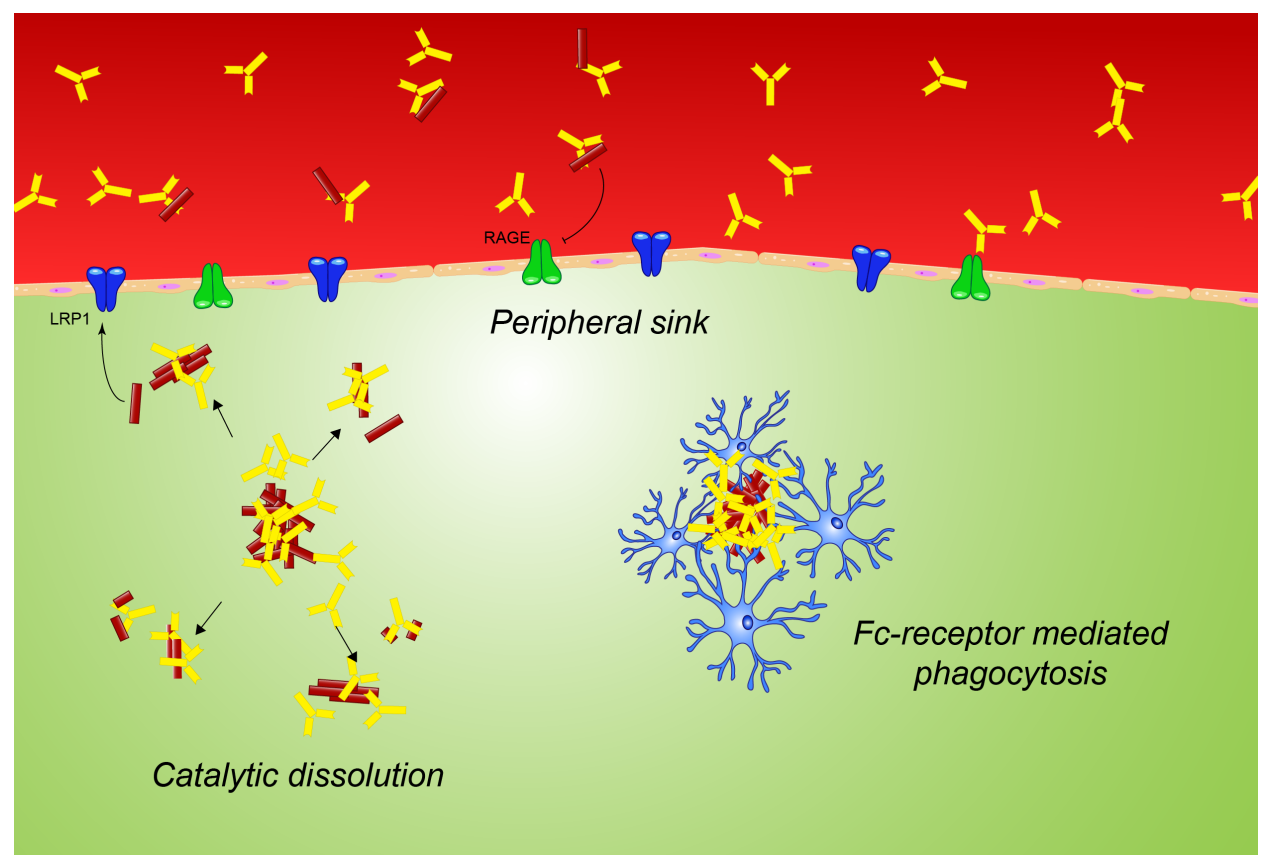

Figure 2.2: Effector mechanisms of anti-A $\beta$ immunotherapy. Several mechanisms for immunotherapy have been proposed. First, antibodies have catalytic activity by themselves and are able to break down plaques into smaller peptides, or allow soluble $A \beta$ to be removed from the brain by means of the LRP1 receptor. In a second mechanism, soluble $A \beta$ in the blood could be bound to antibodies which could prevent it from re-entering the brain by means of the RAGE receptor. This would essentially lead to a net efflux of $A \beta$ from the brain. Finally, antibody bound to $A \beta$ plaques could activate microglia and by means of Fc-receptor mediated phagocytosis, the plaque would be engulfed and removed.

\subsubsection{FCR MEDIATED PHAGOCYTOSIS}

A first effector mechanism of $A \beta$ clearance by immunotherapy is Fc-receptor mediated phagocytosis of plaques by microglia. As mentioned earlier, it is not clear if microglia play a beneficial or detrimental role in $\mathrm{AD}$. This controversy regarding the role of microglia in $\mathrm{AD}$ is also found back in research investigating their function during immunotherapy in $\mathrm{AD}$. Microglia have been shown to be able to phagocytose $A \beta$ via various receptors such as TLR4 [83] and the scavenger receptor [84]. It is hypothesized that antibodies opsonize plaques and increase the phagocytosis of plaques by microglia, thereby decreasing total $\mathrm{A} \beta$ burden in the brain.

Schenk et al. suggested already in the first active immunization study in mice that $A \beta$ clearance might be mediated by microglial phagocytosis and offered some support by showing cells from their immunized mice with a microglial phenotype that contained $A \beta$ [85]. A later study performed by Brazil et al. showed that in vitro microglial uptake of $A \beta$ is indeed enhanced after coating plaques with IgG or C1q, but degradation rates in lysosomes remains the same [86]. Backsai et al. subsequently showed by means of in vivo 2-photon imaging that after application of anti-A $\beta$ antibodies more microglia were associated with plaques [87]. Later on, Bard et al. 
observed that phagocytosis of $A \beta$ is dependent on isotype and epitope specificity of the antibody by showing in an ex vivo assay that only antibodies directed against $\mathrm{N}$-terminal epitopes of $A \beta$ are able to clear plaques via FcR-mediated phagocytosis [88]. They also reported that murine IgG2a antibodies, which have a higher affinity for the murine FcR compared to murine IgG1, are better at reducing plaque load compared to IgG1 antibodies [88]. An overview of the effector functions of the different mouse and human isotypes is presented in Table 2.1. Wilcock et al. showed at the same time that clearance of $A \beta$ in the mouse brain through intracranial injection of an (IgG1) antibody resulted in three different phases of clearance: a first phase which lasts approximately 24 hours and is microglial independent, a second, longer phase between 24 and 72 hours which is marked by an increase in microglial activation as assessed by CD45 and MHC-II staining, and a third phase which shows a normalization to baseline microglial activation [89]. Interestingly, their results are corroborated by data from the AN-1792 trial, in which post-mortem material of participants shows an initial activation phase, followed by a resolution of activation [90].

In a different study, Wilcock et al. also showed that FcR activation seems to be necessary for efficient removal of compact plaques, but not for diffuse plaques. They found that Fab-fragments, which do not bind the FcR, where less efficient in plaque clearance than whole IgG. The same result was found when microglial activation was inhibited with dexamethasone. No difference was found for diffuse plaques clearance, which seems to be mediated in an FcR independent way [91]. Furthermore, in a follow-up study using systemic injection of antibody, they confirmed that microglia cells show more FcR expression after 1 month of antibody administration, and more CD45-expression after 2 months of administration, while after 3 months these markers returned to normal levels together with a significant reduction of $A \beta$ load in the brain [92].

All in all, these experiments demonstrate that microglia phagocytosis has an important contribution to plaque clearance [88].

\subsubsection{PERIPHERAL SINK}

The peripheral sink hypothesis states that immunotherapy changes the $A \beta$ influx/efflux equilibrium to the brain. Antibodies bound to $A \beta$ in the periphery disturb their influx back into the brain, resulting in a net increase of $A \beta$ efflux. The overall result is less $A \beta$ in the brain. The peripheral sink hypothesis was first coined by DeMattos et al. while studying the m266 antibody for immunotherapy (now in trial as solanezumab) [93, 94]. M266, binds to the mid-region of $A \beta$ (AA 13-28) and has a high affinity for soluble monomers [95]. In these studies, DeMattos showed that administration of m266 in PDAPP mice caused a rapid increase in plasma $A \beta$ levels followed by a decrease in brain $A \beta$ load [93]. Since transgenic mice only express human $A \beta$ in the brain, this result could only be explained by the fact that peripheral administration of the antibody caused an increase in brain efflux [93]. Furthermore, in a follow-up experiment they also show that there is a correlation between plasma $A \beta$ levels and total $A \beta$ brain burden after administration of $m 266$, suggesting that plasma $A \beta$ levels after immunotherapy could be used as a serum-based biomarker to assess amyloid status [94].

It should be noted that the results from DeMattos et al. have failed to be replicated 
by a different group. Seubert et al. find no reduction in brain $A \beta$ load after administration of m266 antibody [96]. They do find elevated plasma levels of A $\beta$ after m266 administration, which might have been caused by the fact that $\mathrm{m} 266$ increases the serum half-life of $A \beta$. Combined with the high-turnover rate of $A \beta$, this might explain the increase in serum $A \beta$. A possible explanation for these conflicting results might be found in the animal model, which shows variable plaque accumulation between animals, a fact also noted by DeMattos [96]. The fact remains that the rapid elevation in plasma $A \beta$ levels might indeed be due to an increase in serum-half-life of $A \beta$ as this has also been reported by other groups [97]. Interestingly, Dodart et al. report improvement in cognitive performance of animals without any reduction in brain $A \beta$ load after administration of m266 in an acute treatment model, where animals were tested one day after antibody administration [98].

The peripheral sink effect is probably mediated through the Receptor for Advanced Glycation End-products (RAGE). RAGE has $A \beta$ affinity in the nanomolar range and blockage of RAGE suppresses $A \beta$ influx into the brain [99]. Recently, it has been shown that a peripheral sink action of an antibody through RAGE is also epitope specific [100]. Bachmeier et al. showed using an in vitro BBB model expressing RAGE that $\mathrm{N}$-terminal antibodies are better at preventing $A \beta$ influx into the brain across the RAGE receptor. C-terminal directed antibodies had no effect at all, while mid-terminal antibodies had a mild effect [100]. It would be interesting to see how Solanezumab performs in this experiment, or whether there is some indication that the peripheral sink effect is dependent on other mechanisms besides RAGE transport.

While these studies support the peripheral sink mechanism, a recent study sheds some doubt about this hypothesis. By utilizing neprilysin to degrade $A \beta$ in the periphery, Henderson et al. showed that in monkeys, rats, and non-human primates, $A \beta$ in the periphery is degraded without any effect on $A \beta$ levels in the CNS [101]. An alternative explanation might be that antibodies targeting soluble forms of $A \beta$, prevent $A \beta$ from aggregating into more toxic oligomers by binding to it and increase the efflux of $A \beta$ from the brain [102]. This would also explain the plasma spike of $A \beta$ seen after immunotherapy with some antibodies. A possible mechanism by which antibodies increase $A \beta$ efflux from the brain could be by transport of $A \beta$ bound to antibodies by means of the neonatal Fc-receptor (FcRn) [103].

\subsubsection{CATALYTIC DISSOLUTION}

One of the simplest putative effects of immunotherapy is the catalytic dissolution of plaques or the prevention of fibril formation. The reason why this mechanism is simple, is because it is a direct effect of the antibody and not dependent on other processes such as microglial activation. Several experiments showed that anti-A $\beta$ antibodies could prevent fibrillogenesis of $A \beta$ in vitro $[104,105,106,107,108,109]$ and application of Fab fragments in vivo support this hypothesis [110]. Furthermore, the ability of an antibody to prevent fibril formation also seems to be dependent on the affinity of the antibody [105] and the recognition site in $A \beta$ (epitope). In a direct comparison study between the 3D6 ( $\mathrm{N}$-terminal) and m266 (mid-terminal) antibody, both antibodies performed equally well in a Thiovlavin $\mathrm{T}$ binding assay, showing that both antibodies prevented beta-sheet structure formation. Analysis of this effect by atomic force 
microscopy revealed that $\mathrm{m} 266$ was more effective in preventing fibril formation [111]. Besides full antibodies, also scFv fragments, have been shown to prevent fibril formation and prevent toxicity [112].

Antibodies might also catalyze the direct dissolution of plaques or fibrils. The strongest example of this process has been shown with light chains that directly cleave $A \beta$, thereby preventing the formation of cytotoxic plaques [113]. Specifically, it has been shown that naturally occurring IgM antibodies exert proteolytic activity toward $A \beta$ and that catalytic activity of autoantibodies increases with age [114, 115]. Anti-A $\beta$ antibodies can be isolated from IVIG which is a cocktail of human IgG isolated from a large amount of healthy donors, and usually applied in autoimmune diseases because of its anti-inflammatory properties. These anti-A $\beta$ antibodies from IVIG have been shown to not only prevent fibril formation, but also dissolve existing plaques [104].

\subsection{BLOOD BRAIN BARRIER}

One of the main issues with targeting the brain is delivery of pharmaceuticals across the BBB. While extensive efforts have been directed towards the delivery of small molecules in the brain, there has been surprisingly little work done on how antibodies can exert their therapeutic effects in the brain of Alzheimer patients [116].

The BBB is a network of astrocytes surrounding the blood vessels of the brain combined with tight-junction of vessel endothelial cells that restrict the entry of molecules into the brain. Especially passage of large molecules is limited, unless there is a particular transporter system in place for these molecules. Research has shown that antibodies cross the BBB by means of the extracellular pathway [117]. The extracellular pathway is a collection of leaky vessels mostly located around the circumventricular organs [118]. This influx of antibodies is offset by an active transport mechanism present at the blood brain barrier that transports IgG back to the periphery.

There is conflicting evidence about the role of the neonatal Fc receptor ( $F c R n)$ regarding IgG efflux of the brain. Deane et al. showed that the FcRn is necessary for anti-A $\beta$ IgG mediated efflux from the brain, using a combination of $A D$ mice and FcRn knockouts [103]. Another study, using human monoclonal antibodies with different affinities for the FcRn also showed involvement of the FcRn in rat [119]. Other studies, also using FcRn and Fc-gamma receptor knockouts, showed no involvement of the FcRn in antibody efflux from the brain $[120,121]$. Possible explanations for these discrepancies might be due to different administration methods (systemic and intracranially) or the reduced antibody half-life in FcRn knockout mice.

An additional factor that influences the penetration of antibodies into the brain of $\mathrm{AD}$ patients is the fact that the $\mathrm{BBB}$ in $\mathrm{AD}$ patients is more leaky [122]. This increased permeability is likely caused by factors such as hypoxia, inflammation in the AD brain and amyloid itself [123]. Furthermore, experimental evidence in animal models show that this altered permeability results in decreased $A \beta$ efflux and increased $A \beta$ influx, possibly resulting in further progression of the disease [124]. An increased permeability might make it easier for therapeutic antibodies to reach the brain. One animal study shows that BBB integrity was restored after active immunization, suggesting that once the amyloid pathology has been resolved, BBB permeability is restored to normal levels [125]. Nevertheless, it is important to keep in mind that even when the BBB is intact, 
about $1 \%$ of circulating antibodies are present in the brain [126].

\subsection{CLINICAL RESULTS OF ANTI-A $\beta$ IMMUNOTHERAPY}

Besides the extensive research performed in several $\mathrm{AD}$ animal models, at least 25 immunotherapies against $A \beta$ with a total of over 25000 patients have progressed to clinical trials (see Table 2.2 and Table 2.3). So far the results from the first wave of these trials have shown little benefits for the patients due to either too advanced disease status of the patients, heterogeneity of the patient cohort or unsuitable effector functions of the therapeutic antibodies, or a combination of the aforementioned. Nevertheless, very valuable information has been obtained from them and modified, improved strategies are currently ongoing. In the following sections, results from human clinical trials are summarized. Active immunization trials that have been conducted can be found in Table 2.2 and are discussed below. Subsequently, in Table 2.3 ongoing and completed passive immunization studies are presented.

\subsubsection{ACTIVE IMMUNIZATION}

A first prove of concept that immunization might be a viable treatment strategy was provided by Schenk et al. over 15 years ago in their seminal paper on active immunization with A $\beta 42$ in the PDAPP mouse model, overexpressing mutated human APP [85]. This vaccination was tested in patients, after successfully completing a phase I trial, but the drug was halted in a phase IIa trial (called AN-1792) after $6 \%$ of the patients developed meningoencephalitis [127]. The active immunization protocol consisted of repeated vaccination with full-length A 342 administered with the QS-21 adjuvant to boost the immune response. QS-21 is a emulsion based adjuvant that increases antibody response, but also T-cell response [128].

Although an initial study reported slowing of cognitive decline in responders [129], later studies showed that even though $A \beta$ plaques were removed, cognitive deterioration was not halted [130]. A long-term follow-up study of AN-1792 participants, performed almost 5 years after vaccination, did show significant slowing of decline on some cognitive and functional scales, suggesting there might be some long-term benefits to vaccination [131].

From a biological point of view, much was learned from this vaccination study. The majority of the antibodies generated were directed against the N-terminus of $A \beta$ [132]. This might pose an issue since it is known that up to $60 \%$ of $A \beta$ species in patients has a modified or truncated $\mathrm{N}$-terminus and this might therefore offer an explanation for the absence of a strong effect [133]. Additionally, it was also observed that immediately following immunization, $A \beta$ is solubilized and transported from the parenchyma to the white matter and the vasculature [134, 135]. Interestingly, $A \beta 42$ levels in the brain were lowered in immunized patients as assessed by immunohistochemistry [134], but ELISA measurements in two patients showed no differences in A $\beta$ load [135]. Whether this reduction is a beneficial or harmful effect is currently unknown. Besides a reduction in $\mathrm{A} \beta 42$ in the brain, also a reduction in phospho-tau was observed, although this reduction was confined to neuronal processes and not to the cell body [136].

Initial imaging studies shortly after the vaccination showed an increase in 
ventricular volume [137], which has been attributed to faster loss of already damaged neurons due to increased microglial activity [138]. This finding would also be consistent with the fact that long-term follow-up studies show no increased cerebral atrophy in the immunized patients compared to controls [131]. Another study from the group also shows that immediately following vaccination inflammation is increased, while long-term follow-up shows that inflammatory markers are reduced beyond observed levels at similar AD stages [90].

Sterile meningoencephalitis observed in $6 \%$ of the cases has been attributed to possible activation of cytotoxic T-lymphocytes, a finding which later has also been confirmed in one case study [139]. This aberrant activation has been attributed to the use of the QS-21 adjuvant which can also stimulates T-cell activation [140].

At the moment, new active vaccination therapies that make use of different adjuvants are under investigation, with as goal in mind to generate a sufficiently strong immune response without causing aberrant T-cell activation, thereby making them more safe to use [82]. One of these, CAD106, developed by Novartis, has been tested extensively in patients and immunizes with $A \beta_{1-6}$ [141]. So far, the trials are continuing without any cases of meningoencephalitis or safety problems. Preliminary analysis from Phase IIa trials in 45 patients shows no cognitive benefits as assessed by the ADAScog (Alzheimer's disease assessment scale-cognitive subscale) [142]. It is important to note that these trials were underpowered to detect any possible cognitive effects, and were solely conducted to assess safety and tolerability.

Results from a Phase IIa trials with the active vaccine ACC-001 showed similar results. ACC-001 is an active vaccine making use of A $1-7$ conjugated to a carrier protein and also makes use of the QS-21 adjuvant used in the AN-1792 trial. So far, no safety issues have been reported and patients showed a robust immune response, but exploratory cognitive evaluations also showed no effect of the therapy [143]. This study was also underpowered to detect cognitive benefits, and additionally, no biomarker analysis was done before inclusion of patients, so it is possible that dementia patients without $\mathrm{AD}$ were also included.

Affiris has developed a therapy called Affitope where a peptide resembling $A \beta$ is used to elicit anti-A $\beta$ antibodies. Because of the fact that no self-peptide is used, alum can be used as an adjuvant [144]. Surprisingly, in a Phase II clinical trial testing the Affitope vaccine, patients in the placebo group (AD04, the vaccine without the affitope), performed better than patients in the experimental group (AD02, the vaccine with the affitope) on various cognitive outcome measures such as the ADAScog [145].

ACI-24 is an active liposome based vaccine and results in the generation of antibodies targeting conformational epitopes present on oligomeric A $\beta$ [146]. This vaccine is currently being tested in Phase I/II clinical trial. Although no clinical results have been reported yet, the vaccine is noteworthy because it will be the first immunotherapy which will be tested in patients with Down syndrome.

\subsubsection{PASSIVE IMMUNIZATION}

After the failure of the AN-1792 trial, passive immunotherapy was brought forth as a safe alternative, and in the last decade several passive immunotherapy trials have been conducted. A first trial was conducted with bapineuzumab. Bapineuzumab (IgG1 
isotype) is the humanized form of the murine 3D6 anti-A $\beta$ antibody, which binds the $\mathrm{N}$-terminal of $\mathrm{A} \beta$ and has a high affinity for plaques [147]. 3D6 had previously been shown to be effective in reducing plaque load in the PDAPP mouse model [148, 149]. The phase I trial of bapineuzumab was characterized by the occurrence of Amyloid-Related Imaging Abnormalities (ARIA) [150]. ARIA, sometimes also described as micro-bleeds, is transient in nature and can be detected on a MRI scan. Two types of ARIA are distinguished: ARIA-E, which is thought to represent vasogenic edema and which can be detected by a FLAIR MRI; and ARIA-H, which is related to microhemorrhages and hemosiderosis and can be detected by a T2* weighted MRI scan [151]. What the clinical consequences of ARIA are or even if it poses a health risk for the patient is unknown [152].

In a phase II trial, primary endpoints on cognitive benefits were not met [153]. However, exploratory analysis did show a potential benefit for APOE4 non-carriers on various outcomes such as the ADAScog and other cognitive scales [153]. In addition, amyloid PET scans showed a reduction of $8.5 \%$ in fibrillary amyloid in bapineuzumab treated patients, while there was a concomitant increase of $16.9 \%$ in placebo controlled patients [154].

Analysis showed that risk factors for ARIA were APOE4 status and dose, with more occurrences in the high dose group (2 mg/ $\mathrm{kg}$ ) [151]. Because of these reasons, it was decided to only administer the lowest dose of $0.5 \mathrm{mg} / \mathrm{kg}$ in two phase III trials, with one trial only including APOE4 carriers, and the other trial only including non-carriers [153].

In 2012, the two phase III trials were concluded. Unfortunately, both failed to meet their primary endpoints, and no evidence of clinical benefit was present on any of the applied scales [155]. Suggestions were made that the bapineuzumab dose might have been too low, treatment started too late, or the wrong $A \beta$ species (fibrillary $A \beta$ ) was targeted [155]. Amyloid PET, using 11C-PiB, did show a reduction of fibrillary A $\beta$ [156]. Furthermore, similar to reports from the AN-1792 trial, increases in ventricular volume were reported compared to placebo [157]. Clinical trials using a modified version of bapineuzumab with reduced Fc effector function, called AAB-003, were completed in 2014 , but to date, no results are reported [158, 159]. This modified version of bapineuzumab uses an IgG4 backbone and does not activate complement or binds to Fc-receptors (see Table 2.1) [160].

At approximately the same time as the bapineuzumab clinical phase I trial a solanezumab phase I trial started in parallel. Solanezumab is a humanized IgG1 antibody, (the original mouse is called m266, see section Peripheral sink). Interestingly, the original mouse isotype used in the pre-clinical studies was a mouse IgG1 isotype [93], of which the effector functions would be more comparable to the human IgG4 isotype [161] (see Table 2.1). In contrast to bapineuzumab, solanezumab seems to be better tolerated with lower incidences of ARIA even though the doses used were higher [162]. 
Table 2.1: A comparison of effector functions of the different human and mouse isotypes. The human Fc $\gamma$ RIIB and the mouse $\mathrm{Fc} \gamma \mathrm{RIIB}$ are inhibitory $\mathrm{Fc} \gamma$-receptors, while the other Fc $\gamma$-receptors are activating. The Fc $\gamma \mathrm{Rn}$ (neonatal) is involved in antibody halflife and transport. (Table adopted from [201] and [161])

\begin{tabular}{|c|c|c|c|c|c|}
\hline Human & Isotype & IgG1 & IgG2 & IgG3 & IgG4 \\
\hline & Complement activation & +++ & + & ++ & - \\
\hline & Fc $\gamma \mathbf{R I}$ & ++ & - & ++ & ++ \\
\hline & Fc $\gamma$ RIIA & + & +- & + & + \\
\hline & Fc $\gamma$ RIIB & + & +- & + & + \\
\hline & Fc $\gamma$ RIIC & + & +- & + & + \\
\hline & FçRIIIA & + & +- & + & + \\
\hline & FçRIIIB & + & - & + & + \\
\hline & Fc $\gamma \mathbf{R n}$ & ++ & ++ & + & ++ \\
\hline \multirow[t]{7}{*}{ Mouse } & Isotype & IgG1 & IgG2a & IgG2b & IgG3 \\
\hline & Complement activation & + & ++ & +++ & ++ \\
\hline & Fc $\gamma \mathbf{R I}$ & - & ++ & + & +- \\
\hline & Fc $\gamma$ RIIB & + & + & + & - \\
\hline & Fc $\gamma$ RIII & + & + & + & - \\
\hline & FçrIV & - & ++ & ++ & - \\
\hline & Fc $\gamma R n$ & ++ & ++ & ++ & ++ \\
\hline
\end{tabular}

Shortly after the bapineuzumab results were announced, the results from two phase III trials with solanezumab were also published. Results showed that again primary endpoints as assessed by the ADAS-cog were not reached [163]. However, patients with mild AD (Mini Mental State Exam (MMSE) score between 20 and 26) showed a small, but significant benefit (34\% reduction in decline) on the ADAScog and the MMSE [164].

For the first time in the AD field, a delayed-start trial is being performed on patients that participated in the phase III solanezumab trials. In the delayed-start trial, all patients that participated in the study, including those from the placebo group, will receive solanezumab. Patients and physicians are still blinded to their original condition however. If, at the end of the trial, the difference in cognitive deterioration between the original placebo group and experimental group remains, there is a true treatment effect of the drug. The midterm evaluation from this trial shows that for mild AD patients, the early treatment benefit remains and thus it seems likely that solanezumab has true disease modifying effects, while also lending some evidence that treatments need to start early in the disease process [165]. At the moment, Solanezumab is being investigated in a phase III study with only mild $\mathrm{AD}$ patients and in a parallel preventive trial organized by the Dominantly Inherited Alzheimer Network (DIAN) where it will be administered to patients with familial AD before the onset of symptoms [166, 167].

Gantenerumab is an IgG1 anti-amyloid antibody [168]. Contrary to bapineuzumab and solanezumab, it is a fully human antibody generated by phage display. It binds $\mathrm{N}$ terminally to $A \beta$ with a high affinity for amyloid fibrils, and therefore has similar binding characteristics as bapineuzumab [168]. In a phase II trial, it has been shown to show dose-dependent reduction of $A \beta$ as assessed by PiB PET scans [169]. Like bapineuzumab, 
it also shows problems with ARIA [169]. Gantenerumab was tested in two trials: a phase III trial and a prevention trial (DIAN). In winter 2014, the phase III trial was unexpectedly halted after interim analysis showed no cognitive benefit [170]. The antibody is now being continued in a phase III trial using only patients with mild AD [171].

Another antibody being tested is crenezumab from Genentech. In contrast to all other antibodies tested, crenezumab is a humanized antibody with an IgG4 backbone with reduced effector functions and it might have less side-effects such as ARIA [172]. Interestingly, it also binds soluble $A \beta$ monomers similar to solanezumab (also midterminal) and structural analysis has shown it has almost identical binding properties as solanezumab [173]. In principle, isotype effects could now be compared and this might yield valuable information about the role of inflammation in AD. Furthermore, like solenazumab in the DIAN trial, crenezumab is being tested in a preventive trial called the Alzheimer's Prevention Initiative (API), a trial conducted in the Columbia cohort [174]. In 2014, Genentech announced that the antibody failed to meet its primary endpoints in a phase II trial, but the planned API trial will continue [175].

One promising therapeutic antibody is aducanumab. While no reports have been published yet, some promising results have been publically shared. Aducanumab is a fully human IgG1 antibody targeting fibrils and $A \beta$ oligomers and binds to epitope $A \beta_{3-6}$ $[176,177]$. It has been isolated from a healthy centennial by NeuroImmune and is now being trialed by Biogen Idec. In a phase I trial, the antibody showed a dose dependent slowing of cognitive decline on several cognitive assessment scales. While this group was small, the results looked very promising. One possible caveat was the high incidence of ARIA-E with the antibody. The antibody is now progressing directly to a phase III trial [178].

Another trial, BAN2401, uses a humanized monoclonal antibody targeting $A \beta$ protofibrils. Protofibrils occur after oligomerization of $A \beta$ and have been shown to be possibly more neurotoxic than fibrils [179]. Phase I results from this antibody showed a good safety profile and the therapy is currently under further development [180]. Interestingly, the Phase II study will make use of an innovative trial design based on Bayesian statistics [181]. This design involves frequent interim analyses and changing the randomization procedure based on the outcome of this analysis during the trial. This should allow for both a reduction in the size and the duration of the trial, because group sizes of eg. placebo or certain doses are decreased or increased based on the interim analysis.

An interesting alternative for the use of monoclonal antibodies is the use of intravenous immunoglobulin (IVIG). The mechanism of action for treatment of AD is presumed to depend on the occurrence of natural antibodies present in healthy people [182] including natural anti- $\beta$ antibodies [104]. Trials using IVIG have been modest in the numbers of patients enrolled. Four phase I and II trials enrolling between 6 and 24 patients showed very good results, with an improvement on various outcome measures or a stabilization of cognitive decline [183]. However, a larger phase II trial with 55 patients and a phase III trial with 390 patients provided no proof for cognitive benefit [183]. At the moment, IVIG is being tested in various clinical trials in patients with mild $\mathrm{AD}$ or prodromal stages. An exploratory study in a small group of mild cognitive impairment (MCI) patients suggests that IVIG can delay the onset of $\mathrm{AD}$ by ast least 1 
year [184].

\subsection{ADDITIONAL SAFETY CONCERNS IN IMMUNOTHERAPY}

Besides the meningoencephalitis cases observed in the AN1792 trial, there are a number of other concerns related to amyloid immunotherapy. The most observed safety concern in immunotherapy is of course ARIA. As described earlier, there are two types of ARIA: ARIA-E and ARIA-H (see section "Passive immunization"). Little is known about the long-term effects of ARIA, but they are detected in $A \beta$ immunotherapy studies in both humans and rodents. Mouse studies already suggested that the occurrence of ARIA is dependent on the $A \beta$ form being recognized by the antibodies (soluble versus insoluble) [185], and this is confirmed in the human clinical trials, where none to low incidences of ARIA were reported in the solanezumab and crenezumab trials, which both targeted soluble forms of $A \beta[175,164]$. The exact cause of ARIA is unknown, but it is probably triggered by removal of vascular $A \beta$, causing a temporary increase in vascular permeability [186]. This is further supported by the fact that antithrombotic agents increase the risk for ARIA-H [187]. Additionally, mouse studies show that $A \beta$ immunotherapy can result in increased matrix metalloproteinase activation, occasioning degradation of the extracellular matrix [188].

An often overlooked point is that plaque dissolution might be detrimental to $\mathrm{AD}$ progression in patients. Although still no consensus has been reached on the most toxic form of $A \beta$, there is a general tendency to attribute the strongest toxic effects to the (proto-)fibrillar and oligomeric forms of $A \beta$ [189]. In this view, plaques are considered a neutralized form of $A \beta$ that is actually protective since they clear away (accumulate) the toxic forms. By dissolving the plaques, immunotherapy might actually increase neurotoxicity.

One study found that while patients from the AN1792 trial had decreased A $\beta$ plaques, soluble $A \beta$ in the form of dimers was increased compared to patients receiving placebo [190]. Furthermore, autoantibodies against $A \beta$ isolated from patients may promote aggregation and increase neurotoxicity [191], and anti-oligomeric antibodies (but not monomeric) increase microglial mediated inflammation and subsequent neurotoxicity [192].

Additionally, immunotherapy might also increase neuroinflammation. Results from a previous study show that TNF- $\alpha$, a pro-inflammatory cytokine, is increased in brain after active immunization [190]. Studies in mouse models seem to suggest that active immunotherapy results in a biphasic response, showing increased expression of pro-inflammatory cytokines in early phases after vaccination, while inflammation is dampened in later phases [193].

More worrying are the results from a recently published mouse study failing to show any cognitive improvement using the murine variant of solanezumab (m266) and an oligomer specific antibody (1C22) [194]. Surprisingly, they observe an increase in $20 \%$ mortality rate in antibody treated $\mathrm{AD}$ mice, which might be related to increased excitatory neuronal activity caused by binding of $A \beta$ to the NMDA receptor resulting in deadly convulsions [195]. 


\subsection{CONCLUSIONS AND FUtURE PERSPECTIVES}

So far, most clinical trials with anti-A $\beta$ antibodies have reported negative results. However, 12 different therapies are still ongoing. Of these, solanezumab showed some promise in mild $\mathrm{AD}$ patients and also of aducanumab impressive results were reported on conferences.

The discrepancy between results obtained in $\mathrm{AD}$ animal models and patients, suggests that our current $\mathrm{AD}$ models are not sufficiently similar to the pathogenesis of human $\mathrm{AD}$. One big difference between $\mathrm{AD}$ mouse models and human $\mathrm{AD}$ patients, is that many while many models have $A \beta$ pathology, very few have tau pathology.

One study using direct intrathecal injection of anti-A $\beta$ antibodies showed that targeting $A \beta$, when plaques are already developed, results in reduction of tau, but not of hyperphosphorylated tau aggregates in the 3xTg mouse model, which expresses both hAPP and mutant tau, resulting in plaques and tangles [196]. Only when the therapy was initiated before hyperphosphorylated tau was present, could tau tangles be prevented. The same result was confirmed in an active vaccination study in the same model using an amyloid oligomer vaccine [197]. These results are in concordance with those from clinical trials, showing that immunotherapy reduces plaques, but only has a limited effect on tau tangles [136].

A lesson learned is that $(A \beta)$ immunotherapy should start earlier. Since clinical symptoms of AD correlate better with tau tangles [198], the question remains whether it will ever be possible to start treatment early enough in sporadic $\mathrm{AD}$ patients. If a patient has already memory problems, plaques and tangles are by now present, and probably it is at this time too late for anti-A $\beta$ immunotherapy to have an effect, since so far only limited effects on tau have been shown. These questions will soon be addressed when the various prevention trials are finished. Maybe a combination trial, with antibodies targeting both $\mathrm{A} \beta$ and tau might provide a solution for this conundrum.

Another important aspect to consider is that passive immunotherapy is economically costly. The likelihood that immunotherapy only works in very early stages, means that patients will most likely need to receive antibodies for several years on a regular basis, increasing the financial burden. An additional problem is that patients might generate neutralizing the antibodies against the treatment they receive, thereby rendering the therapy less effective [81].

The anti-amyloid therapy road has broadened our knowledge about $A \beta$ and its pathogenesis. Despite the fact that various trials have ended in negative results, some strategies still show promise as a potential AD treatment. These trials have taught us that it is necessary to define the target group better (e.g. individuals at risk, MCI, familiar forms of $\mathrm{AD}$ ), as well as to consider the antibody effector functions and specificity for the future. New immunotherapy targets are already in progress, with various anti-tau clinical trials currently started. Finally, pre-clinical research might also offer some other targets, such as anti-APOE4 therapy specifically for APOE4 carriers or anti-IL-23 therapy $[199,200]$. 
Table 2.2: Active immunotherapy studies listed in www.clinicaltrials.gov. When a clinical trial is still ongoing, the expected end date is listed. When a clinical trial has been prematurely ended, the term terminated is used. The following abbreviations are used: Clinical trial Phase: Obs-observational Imaging measures: vMRI-volumetric magnetic resonance imaging; ARIA-amyloid related imaging abnormalities; PiB PET-Pittsburgh Compound B PET (amyloid PET); FDG-PETFludeoxyglucose PET (glucose metabolism) Safety related: ECG-Electrocardiogram; PK-pharmacokinetic; ATA-Anti-therapy antibodies Cognition/functional tests: CANTAB-Cambridge Neuropsychological Test Automated Battery; ADCS-PACC-Alzheimer's Disease Cooperative Study-Preclinical Alzheimer's Cognitive Composite; CDCS-CGIC-Clinician Global Impression of Change; CDR-Clinical Dementia Rating; CDR-GS;Clinical Dementia Rating-global score; C-SSRS-Columbia Suicide Severity Rating Scale; DAD-disability assessment for dementia; DIAN-TU-Dominantly Inherited Alzheimer Network Trial; EQ-5D-EuroQol Five Dimensions Questionnaire; FCSRT-Free and Cued Selective Reminding Test; GDS-Geriatric Depression Scale; QoL-AD-Quality of Life-AD; RUD-Lite-Resource Utilization in Dementia; WAIS-R-Wechsler Adult Intelligence Scale; ZCI-AD-Zarit Caregiver Interview for AD

\begin{tabular}{|c|c|c|c|c|c|c|c|c|c|c|}
\hline Therapy & Company & Adjuvant & Antigen & Trial Listing $\mathrm{F}$ & Phase & Status & Primary endpoints & Secondary Endpoints & Patients & Inclusion \\
\hline AN1792 & Janssen/Pfizer & QS21 & $A \beta 1-42$ & NCT00021723 & 2 & Terminated & Not provided & Not provided & 375 & \\
\hline \multirow[t]{6}{*}{ CAD106 } & \multirow[t]{6}{*}{ Novartis } & \multirow[t]{6}{*}{$\begin{array}{c}\mathrm{Q} \beta \\
\text { bacteriophage }\end{array}$} & \multirow[t]{6}{*}{$A \beta 1-6$} & NCT00411580 & 1 & Completed & Safety, antibody titers & $\begin{array}{c}\text { Immune response, } \\
\text { cognitive tests }\end{array}$ & 58 & $\begin{array}{c}\text { Mild to } \\
\text { moderate } \\
\text { AD (MMSE 16-26) }\end{array}$ \\
\hline & & & & NCT01097096 & 2 & Completed & Safety, AE & $\begin{array}{l}\text { Anti-A } \mathrm{A} \text { IgG, } \\
\text { CSF tau/A } \beta\end{array}$ & 177 & $\begin{array}{c}\text { Mild AD } \\
\text { (MMSE 20-26) }\end{array}$ \\
\hline & & & & NCT00956410 & 2 & Completed & Safety & Immune response & 21 & Open label extension \\
\hline & & & & NCT01023685 & 2 & Completed & Safety & $\mathrm{AE}$, immune response & 24 & Open label extension \\
\hline & & & & NCT00795418 & 2 & Completed & Safety & $\begin{array}{l}\text { Immune response, } \\
\text { cognitive tests }\end{array}$ & 31 & Mild AD (MMSE 20-26) \\
\hline & & & & NCT00733863 & 2 & Completed & Safety & Immune response & 27 & $\begin{array}{c}\text { Mild AD } \\
\text { (MMSE 20-26) }\end{array}$ \\
\hline
\end{tabular}




\begin{tabular}{|c|c|c|c|c|c|c|c|c|c|c|}
\hline Therapy & Company & Adjuvant & Antigen & Trial Listing & Phase & Status & Primary endpoints & Secondary Endpoints & Patients & Inclusion \\
\hline & & & & NCT02565511 & $2 / 3$ & Aug 2023 & Time to MCI, APCC & $\begin{array}{l}\text { Immune response, } \\
\text { CDR-SB, Safety, } \\
\text { RBANS, Ecog, } \\
\text { CSF tau/A } \beta \text {, } \\
\text { amyloid PET, } \\
\text { tau PET }\end{array}$ & 1340 & MMSE > 24, APOE4 \\
\hline \multirow[t]{3}{*}{ AD01 } & Affiris & Alum & Affitope & NCT00711139 & 1 & Completed & Safety & & 22 & \\
\hline & & & & NCT00495417 & 1 & Completed & Safety & $\begin{array}{c}\text { Immunological } \\
\text { and clinical } \\
\text { efficacy }\end{array}$ & 24 & $\begin{array}{c}\text { Mild to } \\
\text { moderate AD } \\
\text { (MMSE 16-26) }\end{array}$ \\
\hline & & & & NCT01225809 & Obs & Completed & Safety & & 17 & $\begin{array}{l}\text { Follow up } \\
\text { extension }\end{array}$ \\
\hline \multirow[t]{4}{*}{$\mathrm{AD} 02$} & Affiris & Alum & Affitope & NCT01093664 & 1 & Completed & Safety & $\begin{array}{l}\text { Immunological } \\
\text { and clinical } \\
\text { efficacy }\end{array}$ & 20 & Booster immunization \\
\hline & & & & NCT00633841 & 1 & Completed & safety & $\begin{array}{l}\text { Immunological } \\
\text { and clinical } \\
\text { efficacy }\end{array}$ & 24 & $\begin{array}{c}\text { Mild to } \\
\text { moderate AD } \\
\text { (MMSE 16-26) }\end{array}$ \\
\hline & & & & NCT00711321 & Obs & Completed & safety & $\begin{array}{l}\text { Immunological } \\
\text { and clinical } \\
\text { efficacy }\end{array}$ & 23 & $\begin{array}{l}\text { Follow up } \\
\text { extension }\end{array}$ \\
\hline & & & & NCT01357629 & Obs & Completed & safety & & 11 & \\
\hline
\end{tabular}




\begin{tabular}{|c|c|c|c|c|c|c|c|c|c|c|}
\hline Therapy & Company & Adjuvant & Antigen & Trial Listing & Phase & Status & Primary endpoints & Secondary Endpoints & Patients & Inclusion \\
\hline \multirow{4}{*}{ AD03 } & \multirow{4}{*}{ Affiris } & \multirow{4}{*}{ Alum } & \multirow{4}{*}{ Affitope } & NCT01117818 & 2 & Completed & $\begin{array}{l}\text { ADASCog and } \\
\text { ASCS-ADL }\end{array}$ & $\begin{array}{c}\text { Cognitive } \\
\text { battery, } \\
\text { CDR-SB, NPI, } \\
\text { vMRI }\end{array}$ & 335 & $\begin{array}{c}\text { Early AD } \\
(\text { MMSE >20) }\end{array}$ \\
\hline & & & & NCT02008513 & 2 & Completed & Safety & Composite cognitive & 194 & $\begin{array}{c}\text { Follow up } \\
\text { observational }\end{array}$ \\
\hline & & & & NCT01309763 & 1 & Completed & ADASCog & & 28 & $\begin{array}{c}\text { Mild AD } \\
\text { (MMSE 20-26) }\end{array}$ \\
\hline & & & & NCT01568086 & Obs & Terminated & Safety & $\begin{array}{l}\text { Immunological } \\
\text { and clinical } \\
\text { efficacy }\end{array}$ & 16 & Follow-up \\
\hline \multirow[t]{8}{*}{ ACC-001 } & \multirow[t]{8}{*}{ Janssen } & \multirow[t]{8}{*}{ QS21 } & \multirow[t]{8}{*}{$\mathrm{A} ß 1-7$} & NCT01284387 & 2 & Completed & Efficacy & $\begin{array}{c}\text { Safety, } \\
\text { biomarkers, } \\
\text { cognitive scales }\end{array}$ & 126 & $\begin{array}{c}\mathrm{AD} \text { and } \\
\text { positive } \\
\text { amyloid PET }\end{array}$ \\
\hline & & & & NCT00960531 & 2 & Terminated & AE, safety & Anti-Aß IgG/IgM & 160 & Long term extension \\
\hline & & & & NCT00955409 & 2 & Completed & $\mathrm{AE}$, safety & Anti -Aß IgG/IgM & 50 & Long term extension \\
\hline & & & & NCT00752232 & 2 & Completed & $\mathrm{AE}$, safety & Anti -Aß IgG/IgM & 40 & AD (MMSE 16-26) \\
\hline & & & & NCT00498602 & 2 & Completed & $\mathrm{AE}$, safety & Anti -Aß IgG/IgM & 160 & AD (MMSE 16-26) \\
\hline & & & & NCT01227564 & 2 & Completed & Amyloid PET & $\begin{array}{c}\text { Biomarkers, } \\
\text { immunogenicity }\end{array}$ & 63 & $\begin{array}{c}\text { Mild AD (MMSE }>=25 \text { ), } \\
\text { amyloid PET }\end{array}$ \\
\hline & & & & NCT01238991 & 2 & Terminated & Safety, vMRI, AE & $\begin{array}{c}\text { Biomarkers, } \\
\text { immunogenicity, } \\
\text { cognitive outcomes }\end{array}$ & 53 & $\begin{array}{c}\mathrm{AD} \\
(\mathrm{MMSE}>10)\end{array}$ \\
\hline & & & & NCT00479557 & 2 & Completed & Safety, vMRI, AE & Anti -Aß IgG/IgM & 86 & AD (MMSE 16-26) \\
\hline
\end{tabular}




\begin{tabular}{|c|c|c|c|c|c|c|c|c|c|c|}
\hline Therapy & Company & Adjuvant & Antigen & Trial Listing & Phase & Status & Primary endpoints & Secondary Endpoints & Patients & Inclusion \\
\hline \multirow{7}{*}{ ACI-24 } & \multirow{7}{*}{ AC Immune } & \multirow{7}{*}{ Liposome } & \multirow{7}{*}{ Aß1-15 (oligomer } & NCT00959192 & 2 & Completed & \multirow{7}{*}{$\begin{array}{l}\text { Safety, vMRI, AE } \\
\text { Safety, } \\
\text { antibody titers }\end{array}$} & Anti -Aß IgG/IgM & 32 & AD (MMSE 16-26) \\
\hline & & & & \multirow{6}{*}{ er) NCT02738450 } & \multirow{6}{*}{$\begin{array}{ll}0 & 1\end{array}$} & \multirow{6}{*}{ Aug 2019} & & CANTAB, RBANS, & \multirow{6}{*}{24} & \multirow{6}{*}{ Down syndrome } \\
\hline & & & & & & & & Vineland, NPI, & & \\
\hline & & & & & & & & Amyloid PET, & & \\
\hline & & & & & & & & & & \\
\hline & & & & & & & & CSF tau/Aß, vMRI, & & \\
\hline & & & & & & & & ARIA & & \\
\hline \multirow{7}{*}{ UB-311 } & United & & & & & & & & & \multirow{3}{*}{$\begin{array}{l}\text { Mild moderate AD } \\
\quad \text { (MMSE15-25) }\end{array}$} \\
\hline & Neuroscience & CpG+Alum & $\mathrm{A} ß 1-14$ & NCT00965588 & 1 & Completed & Safety & Immunogenicity & 19 & \\
\hline & Ltd & & & & & & & & & \\
\hline & & & & NCT01189084 & Obs & Completed & $\begin{array}{l}\text { immunogenicity, } \\
\text { safety }\end{array}$ & Efficacy & 14 & Follow-up \\
\hline & & & & & & & & ADASCog, ADCS-ADL, & & \\
\hline & & & & NCT02551809 & 2 & Dec 2017 & Safety & MMSE, CDR-SB, & 45 & Mild AD (MMSE 20-26) \\
\hline & & & & & & & & NPI & & \\
\hline Lu AF20513 I & H. Lundbeck A/S & Unknown & & NCT02388152 & 1 & May 2017 & $\begin{array}{c}\text { Safety, } \\
\text { antibody titers }\end{array}$ & & 35 & $\mathrm{AD}$ \\
\hline V950 & $\begin{array}{l}\text { Merck Sharp } \\
\text { \& Dohme Corp. }\end{array}$ & ISCOMATR IX+Alum & & NCT00464334 & 1 & Completed & $\begin{array}{c}\text { Safety, } \\
\text { antibody titers }\end{array}$ & & 86 & Mild moderate AD \\
\hline
\end{tabular}


Table 2.3: Passive immunotherapy studies listed in www.clinicaltrials.gov. When a clinical trial is still ongoing, the expected end date is listed. When a clinical trial has been prematurely ended, the term terminated is used. The following abbreviations are used: Imaging measures: vMRI-volumetric magnetic resonance imaging; ARIA-amyloid related imaging abnormalities; PiB PET-Pittsburgh Compound B PET (amyloid PET); FDG-PET-Fludeoxyglucose PET (glucose metabolism) Administration methods: IV-intravenous; SC-subcutaneous; IM-intramuscular Safety related: ECG-Electrocardiogram; PK-pharmacokinetic; ATA-Anti-therapy antibodies Cognition/functional tests: CANTAB-Cambridge Neuropsychological Test Automated Battery; ADCS-PACC-Alzheimer's Disease Cooperative StudyPreclinical Alzheimer's Cognitive Composite; CDCS-CGIC-Clinician Global Impression of Change; CDR-Clinical Dementia Rating; CDR-GS;Clinical Dementia Ratingglobal score; C-SSRS-Columbia Suicide Severity Rating Scale; DAD-disability assessment for dementia; DIAN-TU-Dominantly Inherited Alzheimer Network Trial; EQ-5D-EuroQol Five Dimensions Questionnaire; FCSRT-Free and Cued Selective Reminding Test; GDS-Geriatric Depression Scale; QoL-AD-Quality of Life-AD; RUD-

Lite-Resource Utilization in Dementia; WAIS-R-Wechsler Adult Intelligence Scale; ZCI-AD-Zarit Caregiver Interview for AD

\begin{tabular}{|c|c|c|c|c|c|c|c|c|c|c|c|}
\hline Antibody & Company & Target & Isotype & Trial number I & Phase & Status & Primary endpoints & Secondary endpoints & Patients & Admin & Inclusion \\
\hline \multirow[t]{8}{*}{$\begin{array}{c}\text { Gantenerumab } \\
\text { (RO4909832) }\end{array}$} & \multirow[t]{8}{*}{ Roche } & \multirow[t]{8}{*}{ Plaques } & \multirow[t]{8}{*}{ IgG1 } & NCT00531804 & 1 & Completed & $\mathrm{AE}$, safety, PK & $\begin{array}{l}\text { CSF biomarkers, } \\
\text { clinical efficacy }\end{array}$ & 60 & IV & \\
\hline & & & & NCT02133937 & 1 & Completed & PK & $\mathrm{AE}$ & 31 & SC & Healthy \\
\hline & & & & & & & & Safety, & & & \\
\hline & & & & NCT01636531 & 1 & Completed & Bioavailability & $\begin{array}{c}\text { dose-exposure } \\
\text { relationship }\end{array}$ & 120 & SC & \\
\hline & & & & NCT01656525 & 1 & Completed & $\begin{array}{c}\text { AE, PK, } \\
\text { CSF/plasma }\end{array}$ & $\begin{array}{c}\text { plasma A } \beta, \\
\text { CSF tau, } \\
\text { MMSE, ADAScog }\end{array}$ & 28 & SC & \\
\hline & & & & NCT02051608 & 3 & July 2018 & $\begin{array}{l}\text { Florbetapir, } \\
\text { ADAS-cog, }\end{array}$ & $\begin{array}{l}\text { CSF tau/A } \beta_{42} \text { vMRI, } \\
\text { CDR-SB, MMSE, }\end{array}$ & 389 & & $\begin{array}{l}\text { Preventive trial } \\
\text { in patients with }\end{array}$ \\
\hline & & & & & & & ADCS-ADL & safety & & & familial AD \\
\hline & & & & NCT01760005 & $2 / 3$ & Dec 2019 & DIAN-TU & $\begin{array}{c}\text { PiB-PET, } \\
\text { CSF tau/A } \beta_{42}, \\
\text { vMRI, FDG-PET }\end{array}$ & 210 & SC/IV & $\begin{array}{c}\text { Prodromal AD } \\
\text { (MMSE>24 } \\
\text { and amyloid } \\
\text { PET positive) }\end{array}$ \\
\hline
\end{tabular}




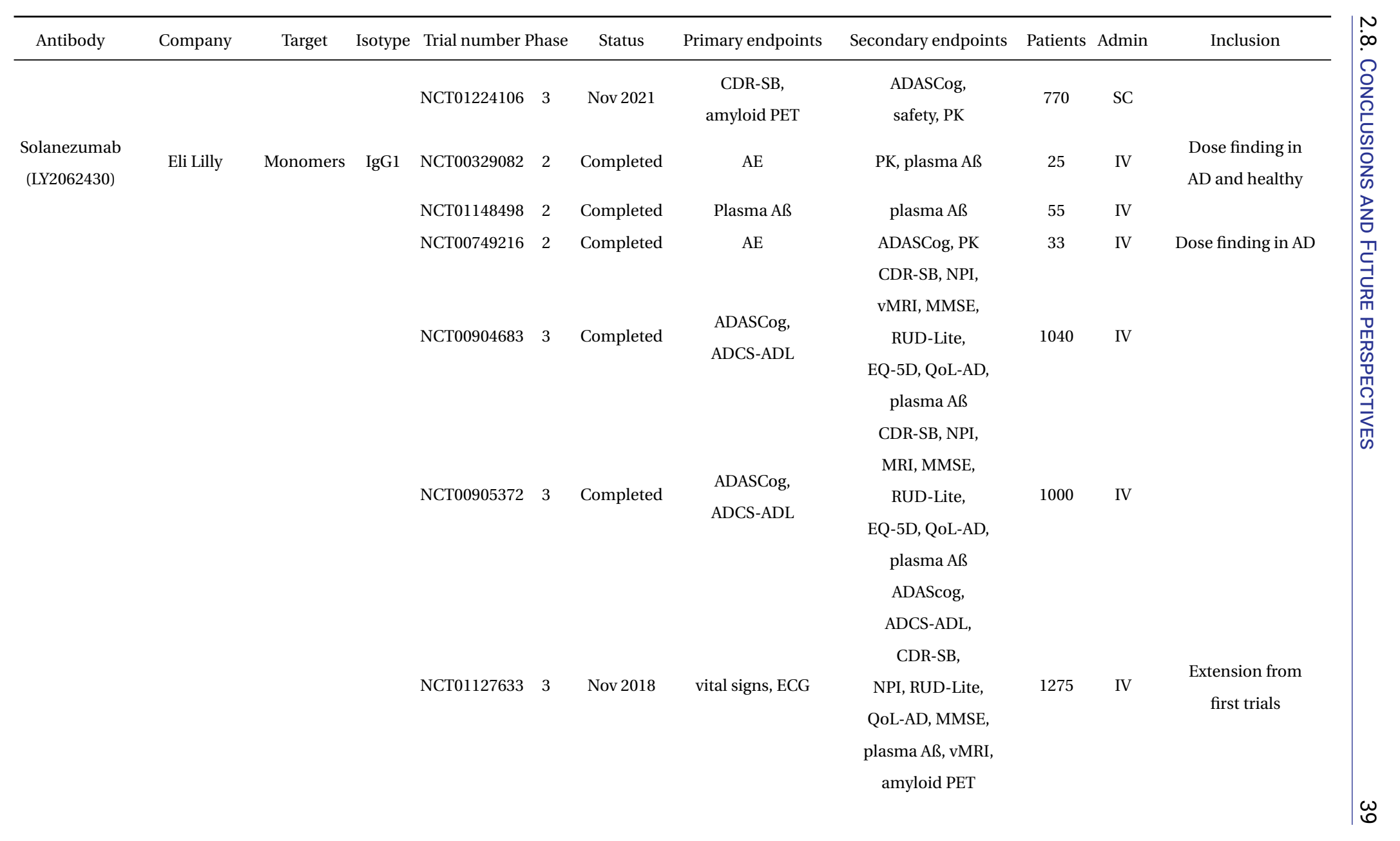




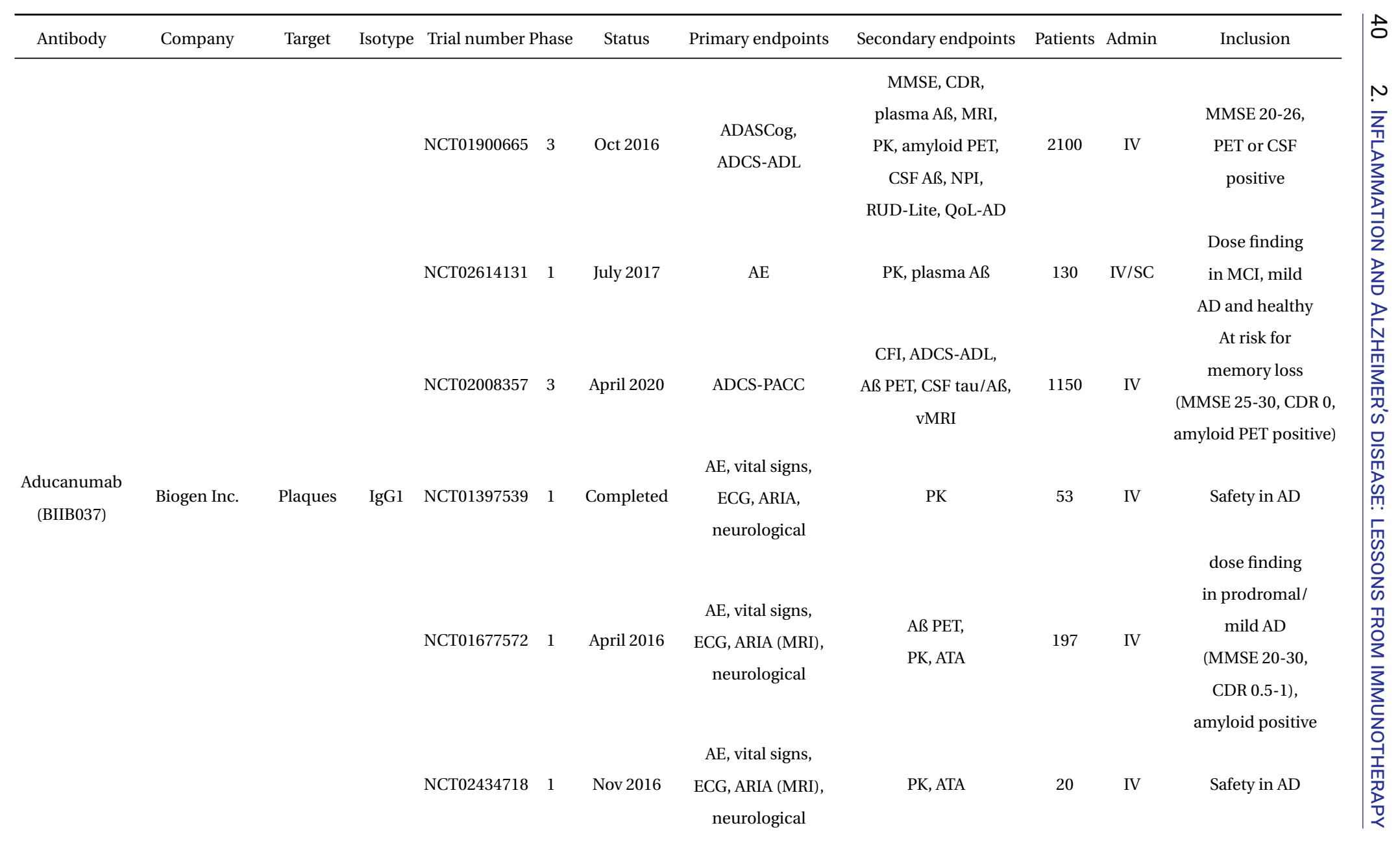




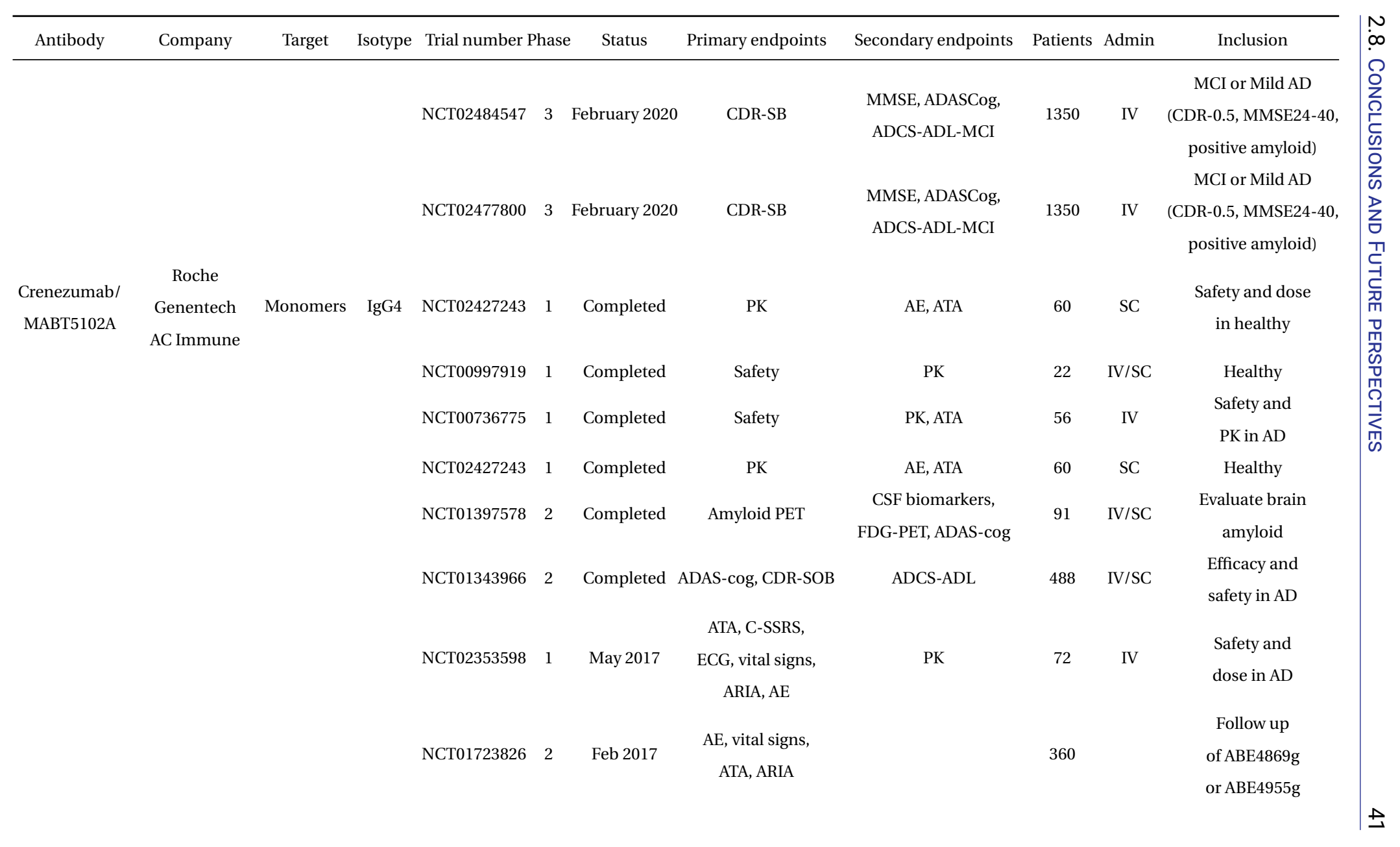




\begin{tabular}{|c|c|c|c|c|c|c|c|c|c|c|c|}
\hline Antibody & Company & Target & Isotype & Trial number $\mathrm{P}$ & hase & Status & Primary endpoints & Secondary endpoints & Patients & Admin & Inclusion \\
\hline \multirow{9}{*}{ Bapineuzumab } & \multirow{9}{*}{$\begin{array}{c}\text { Janssen } \\
\text { Pfizer }\end{array}$} & \multirow{9}{*}{ Plaques } & \multirow{9}{*}{ IgG1 } & NCT01998841 & 2 & Sep 2020 & APCC & $\begin{array}{l}\text { AE, progression } \\
\text { to AD, CDR, CDR-SB, } \\
\text { FCSRT, RBANS, } \\
\text { amyloid PET, } \\
\text { FDG-PET, vMRI, } \\
\text { CSF tau, ATA }\end{array}$ & 300 & SC & $\begin{array}{l}\text { PSEN1 E280A carriers, } \\
\text { MMSE >=24, } \\
\text { does not meet criteria } \\
\text { for dementia or MCI }\end{array}$ \\
\hline & & & & NCT02670083 & 3 & Aug 2020 & CDR-SB & $\begin{array}{c}\text { ADASCog, MMSE, } \\
\text { ADL, CDR-GS, } \\
\text { ADCS-ADL, NPI, } \\
\text { QoL-AD, ZCI-AD, } \\
\text { EQ-5D }\end{array}$ & 750 & IV & $\begin{array}{c}\text { Prodromal and } \\
\text { mild AD } \\
\text { (MMSE }>=22, \\
\text { CDR-GS 0.5-1) CSF or } \\
\text { amyloid PET }\end{array}$ \\
\hline & & & & NCT01193608 & 1 & Completed & $\begin{array}{l}\text { C-SSRS, vMRI, } \\
\text { safety }\end{array}$ & $\begin{array}{c}\text { ATA, ADASCog, } \\
\text { DAD, CDR-SB, } \\
\text { MMSE, CSF tau/Aß, } \\
\text { Plasma Aß }\end{array}$ & 88 & IV & $\begin{array}{l}\text { Safety and } \\
\text { PK in } \mathrm{AD}\end{array}$ \\
\hline & & & & NCT00397891 & 1 & Completed & AE, safety,MMSE & $\begin{array}{c}\text { PK, ATA, } \\
\text { plasma Aß }\end{array}$ & 80 & IV & Safety, $\mathrm{PK}$ in $\mathrm{AD}$ \\
\hline & & & & NCT01254773 & 2 & Completed & $\begin{array}{l}\text { Cerebral } \\
\text { amyloid }\end{array}$ & Safety & 146 & SC & $\mathrm{AD}$ \\
\hline & & & & NCT00663026 & 2 & Completed & AE, safety & PK & 79 & SC & Probable AD \\
\hline & & & & NCT00916617 & 2 & Terminated & AE, safety, vMRI & PK & 66 & SC & Probable AD \\
\hline & & & & NCT00112073 & 2 & Completed & AE, safety & PK & 234 & IV & Safety and PK in $\mathrm{AD}$ \\
\hline & & & & NCT00575055 & 3 & Completed & $\begin{array}{l}\text { Cognitive and } \\
\text { functional }\end{array}$ & $\begin{array}{l}\text { Amyloid imaging, } \\
\text { CSF biomarkers }\end{array}$ & 1121 & IV & APOE4 carriers \\
\hline
\end{tabular}




\begin{tabular}{|c|c|c|c|c|c|c|c|c|c|c|c|}
\hline Antibody & Company & Target & Isotype & Trial number I & Phase & Status & Primary endpoints & Secondary endpoints & Patients & Admin & Inclusion \\
\hline & & & & NCT00574132 & 3 & Completed & $\begin{array}{l}\text { Cognitive and } \\
\text { functional }\end{array}$ & $\begin{array}{l}\text { Amyloid imaging, } \\
\text { CSF biomarkers }\end{array}$ & 1331 & IV & Apoe4 non-carriers \\
\hline & & & & NCT00996918 & 3 & Terminated & AE, safety & $\begin{array}{l}\text { Adas-Cog, DAD, } \\
\text { NPI, MMSE }\end{array}$ & 198 & IV & Phase 3 extension \\
\hline & & & & NCT00667810 & 3 & Terminated & ADASCog, DAD & $\begin{array}{c}\text { Amyloid PET, CSF } \\
\text { tau, vMRI, } \\
\text { ADASCog, DAD, CDR-SOB }\end{array}$ & $\begin{array}{l}901 \\
B^{2}\end{array}$ & IV & In Apoe4 non-carriers \\
\hline & & & & NCT00606476 & 2 & Terminated & AE, safety & $\begin{array}{c}\text { ADASCog, RUDLite, } \\
\text { DAD, MMSE } \\
\text { Amyloid PET, }\end{array}$ & 194 & IV & $\begin{array}{c}\text { Open-label, } \\
\text { long-term extension }\end{array}$ \\
\hline & & & & NCT00676143 & 3 & Terminated & ADASCog, DAD & $\begin{array}{c}\text { CSF tau, } \\
\text { vMRI, CDR-SB }\end{array}$ & 1099 & IV & APOE4 carriers \\
\hline & & & & NCT00998764 & 3 & Terminated & AE, safety, vMRI & $\begin{array}{l}\text { ADASCog, DAD, } \\
\text { NPI, MMSE }\end{array}$ & 494 & IV & $\begin{array}{l}\text { Open-label, } \\
\text { long-term } \\
\text { extension of } \\
\text { APOE4 carriers }\end{array}$ \\
\hline & & & & NCT00937352 & 3 & Terminated & AE, safety, vMRI & Efficacy & 896 & IV & $\begin{array}{c}\text { Open-label, } \\
\text { long-term extension }\end{array}$ \\
\hline & & & & NCT01658722 & 3 & Terminated & Dependence Scale & - & 169 & - & $\begin{array}{c}\text { Follow up } \\
\text { of patients who } \\
\text { discontinued } \\
\text { treatment }\end{array}$ \\
\hline
\end{tabular}




\begin{tabular}{|c|c|c|c|c|c|c|c|c|c|c|c|}
\hline Antibody & Company & Target & Isotype & Trial number $\mathrm{P}$ & hase & Status & Primary endpoints & Secondary endpoints & Patients & s Admin & Inclusion \\
\hline $\begin{array}{c}\text { AAB-003 } \\
(\text { PF-05236812) }\end{array}$ & Janssen/Pfizer & Plaques & IgG4 & NCT01369225 & 1 & Completed & C-SSRS, vMRI, safety & $\begin{array}{c}\text { ATA, ADASCog, } \\
\text { DAD, CDR-SB, } \\
\text { MMSE, CSF tau/Aß, } \\
\text { Plasma Aß }\end{array}$ & 52 & IV & Open label extension \\
\hline \multirow[t]{2}{*}{$\begin{array}{c}\text { N3pG-Aß } \\
\text { (LY3002813) }\end{array}$} & \multirow[t]{2}{*}{ Eli Lilly } & \multirow[t]{2}{*}{$\mathrm{A} \beta_{\mathrm{p} 3-42}$} & \multirow[t]{2}{*}{ Unknowr } & nNCT01837641 & 1 & Sep 2016 & AE, safety & PK & 100 & IV/SC & $\begin{array}{c}\text { Safety and PK } \\
\text { in healthy, MCI } \\
\text { and } \mathrm{AD}\end{array}$ \\
\hline & & & & NCT02624778 & 1 & Aug 2018 & amyloid PET & PK, ATA & 150 & IV & $\begin{array}{l}\text { Effect on brain } \\
\text { amyloid in } \mathrm{AD}\end{array}$ \\
\hline MEDI1814 & AstraZeneca & Oligomers & s Unknowr & nNCT02036645 & 1 & Sep 2016 & $\mathrm{AE}$, safety & PK, ATA & 121 & IV/SC & Safety and $\mathrm{PK}$ in $\mathrm{AD}$ \\
\hline BAN2401 & Eisai Inc & Protofibrils & Is IgG1 & NCT02094729 & 1 & Completed & AE, safety & PK, ATA, CSF tau/Aß & 26 & IV & $\begin{array}{l}\text { Safety in MCI } \\
\text { and mild AD patients } \\
\text { (MMSE 22-30 } \\
\text { and CDR 0.5) }\end{array}$ \\
\hline \multirow{6}{*}{ GSK933776 } & \multirow{6}{*}{ GlaxoSmithKline } & \multirow{6}{*}{ Plaques } & \multirow{6}{*}{ IgG1 } & NCT01230853 & 1 & Completed & Safety & - & 80 & IV & $\begin{array}{l}\text { Safety and dose } \\
\text { finding in } \mathrm{AD}\end{array}$ \\
\hline & & & & NCT01767311 & 2 & Jun 2018 & ADCOMS, AE & vMRI, amyloid PET & 800 & IV & $\begin{array}{l}\text { Clinical efficacy } \\
\text { and dose response }\end{array}$ \\
\hline & & & & NCT01424436 & 1 & Completed & CSF Aß & CSF tau/Aß, Safety & 19 & IV & $\begin{array}{l}\text { MCI or mild AD } \\
\text { (MMSE 20-26) }\end{array}$ \\
\hline & & & & NCT02033668 & 1 & Completed & PK & AE, Safety, ATA & 36 & IV/SC/IM & Healthy \\
\hline & & & & NCT00459550 & 1 & Completed & $\mathrm{AE}$ & PK, CSF, Amyloid PET, ATA & A 50 & IV & Mild AD (MMSE 18-26) \\
\hline & & & & NCT01342926 & 2 & April 2016 & Geohraphic atrophy & visual acuity, PK & 184 & IV & $\begin{array}{c}\text { Age-related } \\
\text { macular degeneration }\end{array}$ \\
\hline
\end{tabular}




\begin{tabular}{|c|c|c|c|c|c|c|c|c|c|c|c|}
\hline Antibody & Company & Target & Isotype & Trial number $\mathrm{P}$ & hase & Status & Primary endpoints & Secondary endpoints & Patients & Admin & Inclusion \\
\hline SAR228810 & Sanofi & Protofibrils & IgG4 & NCT01485302 & 1 & Completed & $\mathrm{AE}$ & PK & 48 & IV/SC & $\begin{array}{l}\text { PK and safety } \\
\text { in } \mathrm{AD}\end{array}$ \\
\hline \multirow[t]{8}{*}{$\begin{array}{l}\text { Ponezumab } \\
\text { (PF-04360365) }\end{array}$} & Pfizer & $\mathrm{A} ß 1-40$ & IgG2a & NCT00733642 & 1 & Completed & Safety & PK & 15 & IV & $\begin{array}{l}\text { PK and safety } \\
\text { in } \mathrm{AD}\end{array}$ \\
\hline & & & & NCT01125631 & 1 & Completed & Safety, PK & Plasma $A ß$ & 8 & IV & $\begin{array}{l}\text { PK and safety } \\
\text { in } \mathrm{AD}\end{array}$ \\
\hline & & & & NCT00607308 & 1 & Completed & Safety & PK & 20 & IV & $\begin{array}{c}\text { PK and safety } \\
\text { in } \mathrm{AD}\end{array}$ \\
\hline & & & & NCT01005862 & 1 & Completed & CSF Aß & $\mathrm{AE}$ & 17 & IV & $\begin{array}{c}\text { Healthy and } \mathrm{AD} \\
\text { patients }\end{array}$ \\
\hline & & & & NCT00455000 & 1 & Completed & Safety, PK & PK, ATA & 37 & IV & $\begin{array}{c}\text { PK and safety } \\
\text { in } \mathrm{AD}\end{array}$ \\
\hline & & & & NCT00945672 & 2 & Completed & $\begin{array}{c}\text { Safety, PK, } \\
\text { brain amyloid, } \\
\text { CSF Aß }\end{array}$ & $\begin{array}{c}\text { ADASCog, } \\
\text { DAD, plasma Aß, } \\
\text { CSF tau/Aß, } \\
\text { ATA }\end{array}$ & 36 & IV & $\begin{array}{c}\text { Safety in } \\
\mathrm{AD}\end{array}$ \\
\hline & & & & NCT01821118 & 2 & Completed & $\begin{array}{l}\text { Cerebrovascular } \\
\text { reactivity }\end{array}$ & plasma $A ß$ & 57 & IV & Safety in CAA \\
\hline & & & & NCT00722046 & 2 & Completed & Safety, PK & $\begin{array}{c}\text { ADASCog, DAD, } \\
\text { plasma Aß, } \\
\text { CSF tau/Aß, } \\
\text { ATA }\end{array}$ & 198 & IV & $\begin{array}{l}\text { Safety and } \\
\text { PK in AD }\end{array}$ \\
\hline
\end{tabular}




\begin{tabular}{|c|c|c|c|c|c|c|c|c|c|c|c|}
\hline Antibody & Company & Target & Isotype & Trial number $\mathrm{P}$ & Phase & Status & Primary endpoints & Secondary endpoints & Patients & Admin & Inclusion \\
\hline Octagam & Octapharma & & IVIG & NCT00812565 & 2 & Completed & plasma $A ß$ & $\begin{array}{c}\text { Anti-Aß antibody, } \\
\text { CSF tau, } \\
\text { ADASCog, } \\
\text { CDR-SOB }\end{array}$ & 58 & IV & Probable AD \\
\hline Gammagard & $\begin{array}{l}\text { Weill Medical } \\
\text { College of } \\
\text { Cornell University }\end{array}$ & & IVIG & NCT00299988 & 2 & Completed & $\begin{array}{l}\text { ADASCog, } \\
\text { CDCS-CGIC }\end{array}$ & $\begin{array}{c}\text { ADCS-ADL, NPI, } \\
\text { GDS, QOL, } \\
\text { ADCS, } \\
\text { Plasma anti-Aß titers, } \\
\text { plasma Aß, FDG-PET, } \\
\text { Amyloid PET, AD }\end{array}$ & 24 & IV & \\
\hline Gammagard & Baxalta US Inc. & & IVIG & NCT00818662 & 3 & Completed & $\begin{array}{l}\text { ADASCog, } \\
\text { ADCS-ADL }\end{array}$ & $\begin{array}{l}\text { ADASCog, ADCS-ADL, } \\
\text { ASCS-CGIC, MMSE, } \\
\text { QOL-AD, WAIS-R, AE }\end{array}$ & 390 & IV & $\mathrm{AD}$ \\
\hline Flebogamma & $\begin{array}{c}\text { Grifols } \\
\text { Biologicals Inc. }\end{array}$ & & $\begin{array}{l}\text { Albumin } \\
\text { and IVIG }\end{array}$ & NCT01561053 & $2 / 3$ & Dec 2016 & $\begin{array}{l}\text { ADASCog, } \\
\text { ADCS-ADL }\end{array}$ & $\begin{array}{c}\text { Cognitive, } \\
\text { CSF tau/Aß, } \\
\text { FDG-PET, } \\
\text { vMRI }\end{array}$ & 350 & IV & $\mathrm{AD}$ \\
\hline NewGam & Sutter Health & & IVIG & NCT01300728 & 2 & Unknown & $\begin{array}{l}\text { vMRI, CDR-SB, } \\
\text { CSF Aß, cognitive } \\
\text { performance }\end{array}$ & - & 52 & IV & $\begin{array}{c}\text { MCI (MMSE } \\
\text { 24-30, CDR 0.5) }\end{array}$ \\
\hline Gammagard & Baxalta US Inc. & & IVIG & NCT01736579 & 3 & Terminated & $\mathrm{AE}$ & Cognition & 6 & IV & Open label extension \\
\hline
\end{tabular}




\section{REFERENCES}

[1] Prince M, Wimo A, Guerchet M, Ali GC, Wu YT, Prina M, et al. World Alzheimer Report 2015. Alzheimer's Disease International (ADI); 2015.

[2] Reitz C, Brayne C, Mayeux R. Epidemiology of Alzheimer disease [Journal Article]. Nature Reviews Neurology. 2011;7(3):137-152.

[3] Bertram L, Lill CM, Tanzi RE. The Genetics of Alzheimer Disease: Back to the Future [Journal Article]. Neuron. 2010;68(2):270-281. Available from: http:// wWw.sciencedirect.com/science/article/pii/S0896627310008378.

[4] Ertekin-Taner N. Genetics of Alzheimer's Disease: A Centennial Review [Journal Article]. Neurologic Clinics. 2007;25(3):611-667. Available from: http://www . sciencedirect.com/science/article/pii/S0733861907000552.

[5] Gatz M, Reynolds CA, Fratiglioni L, et al. Role of genes and environments for explaining Alzheimer disease [Journal Article]. Archives of General Psychiatry. 2006;63(2):168-174. Available from: http://dx.doi.org/10.1001/archpsyc. 63.2 .168$.

[6] Hardy J, Selkoe DJ. The Amyloid Hypothesis of Alzheimer's Disease: Progress and Problems on the Road to Therapeutics [Journal Article]. Science. 2002;297(5580):353-356. Available from: http://www.sciencemag.org/ content/297/5580/353. abstract.

[7] Selkoe DJ, Podlisny MB, Joachim CL, Vickers EA, Lee G, Fritz LC, et al. Betaamyloid precursor protein of Alzheimer disease occurs as 110- to 135-kilodalton membrane-associated proteins in neural and nonneural tissues [Journal Article]. Proc Natl Acad Sci U S A. 1988;85(19):7341-5.

[8] Dawkins E, Small DH. Insights into the physiological function of the $\beta$-amyloid precursor protein: beyond Alzheimer's disease [Journal Article]. Journal of Neurochemistry. 2014;129(5):756-769. Available from: http://dx.doi.org/10 . $1111 /$ jnc. 12675.

[9] Suh J, Choi SH, Romano DM, Gannon MA, Lesinski AN, Kim DY, et al. ADAM10 Missense Mutations Potentiate $\beta$-Amyloid Accumulation by Impairing Prodomain Chaperone Function [Journal Article]. Neuron. 2013;80(2):385401. Available from: http://www.sciencedirect.com/science/article/ pii/S0896627313007940.

[10] Haass C, Kaether C, Thinakaran G, Sisodia S. Trafficking and Proteolytic Processing of APP [Journal Article]. Cold Spring Harbor Perspectives in Medicine. 2012;2(5). Available from: http://perspectivesinmedicine.cshlp.org/content/2/ 5/a006270. abstract.

[11] Bergmans BA, De Strooper B. $\gamma$-secretases: from cell biology to therapeutic strategies [Journal Article]. The Lancet Neurology. 2010;9(2):215226. Available from: http://www.sciencedirect.com/science/article/ pii/S1474442209703321. 
[12] O’Brien RJ, Wong PC. Amyloid Precursor Protein Processing and Alzheimer's Disease [Journal Article]. Annu Rev Neurosci. 2011;34:185-204.

[13] Zetterberg H, Blennow K, Hanse E. Amyloid $\beta$ and APP as biomarkers for Alzheimer's disease [Journal Article]. Experimental Gerontology. 2010;45(1):2329. Available from: http://www.sciencedirect.com/science/article/pii/ S0531556509001594.

[14] Soreghan B, Kosmoski J, Glabe C. Surfactant properties of Alzheimer's A beta peptides and the mechanism of amyloid aggregation [Journal Article]. Journal of Biological Chemistry. 1994;269(46):28551-28554. Available from: http://www . jbc.org/content/269/46/28551. abstract.

[15] Tanzi RE, Bertram L. Twenty Years of the Alzheimer's Disease Amyloid Hypothesis: A Genetic Perspective [Journal Article]. Cell. 2005;120(4):545555. Available from: http://www.sciencedirect.com/science/article/ pii/S0092867405001522.

[16] Pimplikar SW. Reassessing the Amyloid Cascade Hypothesis of Alzheimer's Disease [Journal Article]. The international journal of biochemistry \& cell biology. 2009;41(6):1261-1268. Available from: http://www.ncbi.nlm.nih.gov/pmc/ articles/PMC2680505/.

[17] Walsh DM, Selkoe DJ. A $\beta$ Oligomers - a decade of discovery [Journal Article]. Journal of Neurochemistry. 2007;101(5):1172-1184. Available from: http://dx . doi.org/10.1111/j.1471-4159.2006.04426.x.

[18] Jongbloed W, Bruggink KA, Kester MI, Visser PJ, Scheltens P, Blankenstein MA, et al. Amyloid- $\beta$ Oligomers Relate to Cognitive Decline in Alzheimer's Disease [Journal Article]. Journal of Alzheimer's Disease. 2015;45(1):35-43.

[19] Bayer TA, Wirths O. Focusing the amyloid cascade hypothesis on N-truncated Abeta peptides as drug targets against Alzheimer's disease [Journal Article]. Acta Neuropathologica. 2014;127(6):787-801. Available from: http://dx.doi.org/ $10.1007 / \mathrm{s} 00401-014-1287-\mathrm{x}$.

[20] Doraiswamy PM, Leon J, Cummings JL, Marin D, Neumann PJ. Prevalence and Impact of Medical Comorbidity in Alzheimer's Disease [Journal Article]. The Journals of Gerontology Series A: Biological Sciences and Medical Sciences. 2002;57(3):M173-M177. Available from: http://biomedgerontology . oxfordjournals .org/content/57/3/M173. abstract.

[21] Zempel H, Mandelkow E. Lost after translation: missorting of Tau protein and consequences for Alzheimer disease [Journal Article]. Trends in Neurosciences. 2014;37(12):721-732. Available from: http://www.sciencedirect.com/ science/article/pii/S0166223614001313.

[22] Rhein V, Song X, Wiesner A, Ittner LM, Baysang G, Meier F, et al. Amyloid- $\beta$ and tau synergistically impair the oxidative phosphorylation system in triple transgenic 
Alzheimer's disease mice [Journal Article]. Proceedings of the National Academy of Sciences of the United States of America. 2009;106(47):20057-20062. Available from: http://www.ncbi.nlm.nih.gov/pmc/articles/PMC2774257/.

[23] Seward ME, Swanson E, Norambuena A, Reimann A, Cochran JN, Li R, et al. Amyloid- $\beta$ signals through tau to drive ectopic neuronal cell cycle re-entry in Alzheimer's disease [Journal Article]. Journal of Cell Science. 2013;126(5):12781286. Available from: http://jcs.biologists.org/joces/126/5/1278. full.pdf.

[24] Shipton OA, Leitz JR, Dworzak J, Acton CEJ, Tunbridge EM, Denk F, et al. Tau Protein Is Required for Amyloid BInduced Impairment of Hippocampal LongTerm Potentiation [Journal Article]. The Journal of Neuroscience. 2011;31(5):16881692. Available from: http://www.jneurosci.org/content/31/5/1688. abstract.

[25] Giannakopoulos P, Herrmann FR, Bussière T, Bouras C, Kovari E, Perl DP, et al. Tangle and neuron numbers, but not amyloid load, predict cognitive status in Alzheimer's disease [Journal Article]. Neurology. 2003;60(9):1495-1500. Available from: http://www. neurology.org/content/60/9/1495. abstract.

[26] Farrer LA, Cupples L, Haines JL, et al. Effects of age, sex, and ethnicity on the association between apolipoprotein e genotype and alzheimer disease: A metaanalysis [Journal Article]. JAMA. 1997;278(16):1349-1356. Available from: http: //dx.doi.org/10.1001/jama.1997.03550160069041.

[27] Liu CC, Kanekiyo T, Xu H, Bu G. Apolipoprotein E and Alzheimer disease: risk, mechanisms, and therapy [Journal Article]. Nature reviews Neurology. 2013;9(2):106-118. Available from: http://www.ncbi.nlm.nih.gov/pmc/ articles/PMC3726719/.

[28] Gale SC, Gao L, Mikacenic C, Coyle SM, Rafaels N, Murray Dudenkov T, et al. APOE4 is associated with enhanced in vivo innate immune responses in human subjects [Journal Article]. Journal of Allergy and Clinical Immunology. 2014;134(1):127-134.e9. Available from: http://www.sciencedirect.com/ science/article/pii/S0091674914001961.

[29] Egensperger R, Kasel S, von Eitzen U, Graeber MB. Microglial Activation in Alzheimer Disease: Association with APOE Genotype [Journal Article]. Brain Pathology. 1998;8(3):439-447. Available from: http://dx.doi .org/10.1111/j . 1750-3639.1998.tb00166.x.

[30] Tai LM, Ghura S, Koster KP, Liakaite V, Maienschein-Cline M, Kanabar P, et al. APOE-modulated A $\beta$-induced neuroinflammation in Alzheimer's disease: current landscape, novel data, and future perspective [Journal Article]. Journal of Neurochemistry. 2015;133(4):465-488. Available from: http://dx . doi .org/10 . $1111 /$ jnc. 13072. 
[31] Wyss-Coray T. Inflammation in Alzheimer disease: driving force, bystander or beneficial response? [Journal Article]. Nat Med. 2006;12(9):1005-1015. Available from: http://dx.doi.org/10.1038/nm1484.

[32] Stewart WF, Kawas C, Corrada M, Metter EJ. Risk of Alzheimer's disease and duration of NSAID use [Journal Article]. Neurology. 1997;48(3):626-632. Available from: http: //www . neurology . org/content/48/3/626. abstract.

[33] Vlad SC, Miller DR, Kowall NW, Felson DT. Protective effects of NSAIDs on the development of Alzheimer disease [Journal Article]. Neurology. 2008;70(19):16721677. Available from: http://www.neurology.org/content/70/19/1672. abstract.

[34] Aisen PS, Schafer KA, Grundman M, et al. Effects of rofecoxib or naproxen vs placebo on alzheimer disease progression: A randomized controlled trial [Journal Article]. JAMA. 2003;289(21):2819-2826. Available from: http://dx.doi.org/ 10.1001/jama.289.21.2819.

[35] Scharf S, Mander A, Ugoni A, Vajda F, Christophidis N. A double-blind, placebo-controlled trial of diclofenac/misoprostol in Alzheimer's disease [Journal Article]. Neurology. 1999;53(1):197. Available from: http://www.neurology . org/content/53/1/197. abstract.

[36] Heppner FL, Ransohoff RM, Becher B. Immune attack: the role of inflammation in Alzheimer disease [Journal Article]. Nat Rev Neurosci. 2015;16(6):358-372. Available from: http://dx.doi.org/10.1038/nrn3880.

[37] El Khoury JB, Moore KJ, Means TK, Leung J, Terada K, Toft M, et al. CD36 Mediates the Innate Host Response to $\beta$-Amyloid [Journal Article]. The Journal of Experimental Medicine. 2003;197(12):1657-1666. Available from: http:// jem . rupress .org/content/197/12/1657. abstract.

[38] Stewart CR, Stuart LM, Wilkinson K, van Gils JM, Deng J, Halle A, et al. CD36 ligands promote sterile inflammation through assembly of a Toll-like receptor 4 and 6 heterodimer [Journal Article]. Nat Immunol. 2010;11(2):155-161. Available from: http://dx.doi.org/10.1038/ni.1836.

[39] Hickman SE, Allison EK, El Khoury J. Microglial Dysfunction and Defective $\beta$ Amyloid Clearance Pathways in Aging Alzheimer's Disease Mice [Journal Article]. The Journal of Neuroscience. 2008;28(33):8354-8360. Available from: http:// www. jneurosci.org/content/28/33/8354. abstract.

[40] Morales I, Jiménez JM, Mancilla M, Maccioni RB. Tau oligomers and fibrils induce activation of microglial cells [Journal Article]. Journal of Alzheimer's disease: JAD. 2012;37(4):849-856.

[41] Weitz TM, Town T. Microglia in Alzheimer's Disease: It's All About Context [Journal Article]. International Journal of Alzheimer's Disease. 2012;2012:11. Available from: http://dx.doi.org/10.1155/2012/314185. 
[42] Solito E, Sastre M. Microglia function in Alzheimer's disease [Journal Article]. Frontiers in Pharmacology. 2012;3. Available from: http://www.frontiersin. org/Journal/Abstract . aspx?s=762\&name=neuropharmacology\&ART_DOI= 10.3389/fphar.2012.00014.

[43] Lee CD, Landreth GE. The role of microglia in amyloid clearance from the AD brain [Journal Article]. Journal of neural transmission. 2010;117(8):949-960.

[44] Mawuenyega KG, Sigurdson W, Ovod V, Munsell L, Kasten T, Morris JC, et al. Decreased clearance of CNS $\beta$-amyloid in Alzheimer's disease [Journal Article]. Science. 2010;330(6012):1774-1774.

[45] Medway C, Morgan K. Review: The genetics of Alzheimer's disease; putting flesh on the bones [Journal Article]. Neuropathology and Applied Neurobiology. 2014;40(2):97-105. Available from: http://dx.doi .org/10.1111/nan.12101.

[46] Jiang T, Yu JT, Hu N, Tan MS, Zhu XC, Tan L. CD33 in Alzheimer's Disease [Journal Article]. Molecular Neurobiology. 2013;49(1):529-535. Available from: http:// dx.doi.org/10.1007/s12035-013-8536-1.

[47] Guerreiro R, Wojtas A, Bras J, Carrasquillo M, Rogaeva E, Majounie E, et al. TREM2 Variants in Alzheimer's Disease [Journal Article]. New England Journal of Medicine. 2013;368(2):117-127. Available from: http://www.nejm.org/doi/ full/10.1056/NEJMoa1211851.

[48] Colonna M, Wang Y. TREM2 variants: new keys to decipher Alzheimer disease pathogenesis [Journal Article]. Nat Rev Neurosci. 2016;17(4):201-207. Available from: http://dx.doi.org/10.1038/nrn.2016.7.

[49] Wang Y, Cella M, Mallinson K, Ulrich JD, Young KL, Robinette ML, et al. TREM2 Lipid Sensing Sustains the Microglial Response in an Alzheimer's Disease Model [Journal Article]. Cell. 2015;160(6):1061-1071. Available from: http://www . sciencedirect.com/science/article/pii/S0092867415001270.

[50] Yaghmoor F, Noorsaeed A, Alsaggaf S, Aljohani W, Scholtzova H, Boutajangout A, et al. The Role of TREM2 in Alzheimer's Disease and Other Neurological Disorders [Journal Article]. Journal of Alzheimer's disease \& Parkinsonism. 2014;4(5):160. Available from: http://www.ncbi.nlm.nih.gov/pmc/ articles/PMC4317331/.

[51] Tanzi RE. TREM2 and Risk of Alzheimer's Disease: Friend or Foe? [Journal Article]. New England Journal of Medicine. 2015;372(26):2564-2565. Available from: http: //www.nejm.org/doi/full/10.1056/NEJMcibr1503954.

[52] Rogers J, Cooper NR, Webster S, Schultz J, McGeer PL, Styren SD, et al. Complement activation by beta-amyloid in Alzheimer disease [Journal Article]. Proceedings of the National Academy of Sciences. 1992;89(21):10016-10020. 
[53] Jiang H, Burdick D, Glabe CG, Cotman CW, Tenner AJ. beta-Amyloid activates complement by binding to a specific region of the collagen-like domain of the C1q A chain [Journal Article]. The Journal of Immunology. 1994;152(10):5050-5059.

[54] Fonseca MI, Chu SH, Berci AM, Benoit ME, Peters DG, Kimura Y, et al. Contribution of complement activation pathways to neuropathology differs among mouse models of Alzheimer's disease [Journal Article]. J Neuroinflammation. 2011;8(4).

[55] Li M, Ager RR, Fraser DA, Tjokro NO, Tenner AJ. Development of a humanized Clq A chain knock-in mouse: Assessment of antibody independent $\beta$-amyloid induced complement activation [Journal Article]. Molecular Immunology. 2008;45(11):3244-3252. Available from: http://www.sciencedirect.com/ science/article/pii/S0161589008000990.

[56] Mencarelli C, Losen M, Hammels C, De Vry J, Hesselink MKC, Steinbusch HWM, et al. The ceramide transporter and the Goodpasture antigen binding protein: one protein, one function? [Journal Article]. Journal of Neurochemistry. 2010;113(6):1369-1386. Available from: http://dx.doi.org/10.1111/j . $1471-4159.2010 .06673 . x$.

[57] Mencarelli C, Martinez-Martinez P. Ceramide function in the brain: when a slight tilt is enough [Journal Article]. Cellular and Molecular Life Sciences. 2012;70(2):181-203. Available from: http://dx.doi.org/10.1007/ s00018-012-1038-x.

[58] Bode GH, Losen M, Buurman WA, Veerhuis R, Molenaar PC, Steinbusch HW, et al. Complement activation by ceramide transporter proteins [Journal Article]. The Journal of Immunology. 2014;192(3):1154-1161.

[59] Mencarelli C, Bode GH, Losen M, Kulharia M, Molenaar PC, Veerhuis R, et al. Goodpasture antigen-binding protein/ceramide transporter binds to human serum amyloid P-component and is present in brain amyloid plaques [Journal Article]. Journal of Biological Chemistry. 2012;287(18):14897-14911.

[60] Stevens B, Allen NJ, Vazquez LE, Howell GR, Christopherson KS, Nouri N, et al. The classical complement cascade mediates CNS synapse elimination [Journal Article]. Cell. 2007;131(6):1164-1178.

[61] Benoit ME, Tenner AJ. Complement protein C1q-mediated neuroprotection is correlated with regulation of neuronal gene and microRNA expression [Journal Article]. The Journal of Neuroscience. 2011;31(9):3459-3469.

[62] Fonseca MI, Zhou J, Botto M, Tenner AJ. Absence of Clq leads to less neuropathology in transgenic mouse models of Alzheimer's disease [Journal Article]. The Journal of neuroscience. 2004;24(29):6457-6465.

[63] Matarin M, Salih DA, Yasvoina M, Cummings DM, Guelfi S, Liu W, et al. A Genome-wide Gene-Expression Analysis and Database in Transgenic Mice during Development of Amyloid or Tau Pathology [Journal Article]. Cell 
Reports. 2015;10(4):633-644. Available from: http: //www . sciencedirect.com/ science/article/pii/S2211124714010948.

[64] Shi Q, Colodner KJ, Matousek SB, Merry K, Hong S, Kenison JE, et al. Complement C3-deficient mice fail to display age-related hippocampal decline [Journal Article]. The Journal of Neuroscience. 2015;35(38):13029-13042.

[65] Lian H, Litvinchuk A, Chiang ACA, Aithmitti N, Jankowsky JL, Zheng H. AstrocyteMicroglia Cross Talk through Complement Activation Modulates Amyloid Pathology in Mouse Models of Alzheimer's Disease [Journal Article]. The Journal of Neuroscience. 2016;36(2):577-589.

[66] Fu H, Liu B, Frost JL, Hong S, Jin M, Ostaszewski B, et al. Complement component $\mathrm{C} 3$ and complement receptor type 3 contribute to the phagocytosis and clearance of fibrillar A $\beta$ by microglia [Journal Article]. Glia. 2012;60(6):993-1003.

[67] Maier M, Peng Y, Jiang L, Seabrook TJ, Carroll MC, Lemere CA. Complement C3 deficiency leads to accelerated amyloid $\beta$ plaque deposition and neurodegeneration and modulation of the microglia/macrophage phenotype in amyloid precursor protein transgenic mice [Journal Article]. The Journal of Neuroscience. 2008;28(25):6333-6341.

[68] Eikelenboom P, Hoozemans J, Veerhuis R, van Exel E, Rozemuller A, van Gool WA. Whether, when and how chronic inflammation increases the risk of developing late-onset Alzheimer's disease [Journal Article]. Alzheimers Res Ther. 2012;4(3):15.

[69] Yoon S, Kim YK. The role of immunity and neuroinflammation in genetic predisposition and pathogenesis of Alzheimer's disease [Journal Article]. AIMS Genetics. 2015;2(3):230-249. Available from: http://www.aimspress.com/ Genetics/article/449.html.

[70] Zhu XC, Yu JT, Jiang T, Wang P, Cao L, Tan L. CR1 in Alzheimer's Disease [Journal Article]. Molecular Neurobiology. 2014;51(2):753-765. Available from: http:// dx.doi.org/10.1007/s12035-014-8723-8.

[71] Fonseca MI, Chu S, Pierce AL, Brubaker WD, Hauhart RE, Mastroeni D, et al. Analysis of the Putative Role of CR1 in Alzheimer's Disease: Genetic Association, Expression and Function [Journal Article]. PloS one. 2016;11(2):e0149792.

[72] Rogers J, Li R, Mastroeni D, Grover A, Leonard B, Ahern G, et al. Peripheral clearance of amyloid $\beta$ peptide by complement C3-dependent adherence to erythrocytes [Journal Article]. Neurobiology of aging. 2006;27(12):1733-1739.

[73] Martinon F, Burns K, Tschopp J. The inflammasome: a molecular platform triggering activation of inflammatory caspases and processing of proIL- $\beta$ [Journal Article]. Molecular cell. 2002;10(2):417-426.

[74] Halle A, Hornung V, Petzold GC, Stewart CR, Monks BG, Reinheckel T, et al. The NALP3 inflammasome is involved in the innate immune response to amyloid$\beta[$ Journal Article]. Nature immunology. 2008;9(8):857-865. 
[75] Shaftel SS, Griffin W, O’Banion MK. The role of interleukin-1 in neuroinflammation and Alzheimer disease: an evolving perspective [Journal Article]. J Neuroinflammation. 2008;5(7):28.

[76] Saresella M, La Rosa F, Piancone F, Zoppis M, Marventano I, Calabrese E, et al. The NLRP3 and NLRP1 inflammasomes are activated in Alzheimer's disease [Journal Article]. Molecular Neurodegeneration. 2016;11(1):1-14. Available from: http: //dx.doi.org/10.1186/s13024-016-0088-1.

[77] Heneka MT, Kummer MP, Stutz A, Delekate A, Schwartz S, Vieira-Saecker A, et al. NLRP3 is activated in Alzheimer/'s disease and contributes to pathology in APP/PS1 mice [Journal Article]. Nature. 2013;493(7434):674-678. Available from: http://dx.doi.org/10.1038/nature11729.

[78] Aiyer Harini P, Ashok Kumar H. An Overview of Immunologic Adjuvants-A Review [Journal Article]. Journal of Vaccines \& Vaccination. 2013;2013.

[79] Reed SG, Orr MT, Fox CB. Key roles of adjuvants in modern vaccines [Journal Article]. Nature medicine. 2013;19(12):1597-1608.

[80] Awate S, Babiuk LA, Mutwiri G. Mechanisms of action of adjuvants [Journal Article]. Front Immunol. 2013;4(114):1-10.

[81] Nechansky A. HAHA - nothing to laugh about. Measuring the immunogenicity (human anti-human antibody response) induced by humanized monoclonal antibodies applying ELISA and SPR technology [Journal Article]. Journal of Pharmaceutical and Biomedical Analysis. 2010;51(1):252-254. Available from: http: //www.sciencedirect.com/science/article/pii/S0731708509004725.

[82] Agadjanyan MG, Petrovsky N, Ghochikyan A. A fresh perspective from immunologists and vaccine researchers: Active vaccination strategies to prevent and reverse Alzheimer's disease [Journal Article]. Alzheimer's \& Dementia. 2015;11(10):1246-1259. Available from: http://www.sciencedirect.com/ science/article/pii/S1552526015021214.

[83] Tahara K, Kim HD, Jin JJ, Maxwell JA, Li L, Fukuchi Ki. Role of toll-like receptor signalling in A $\beta$ uptake and clearance [Journal Article]. Brain. 2006;129(11):30063019. Available from: http://brain.oxfordjournals.org/content/129/11/ 3006. abstract.

[84] Wilkinson K, El Khoury J. Microglial Scavenger Receptors and Their Roles in the Pathogenesis of Alzheimer's Disease [Journal Article]. International Journal of Alzheimer's Disease. 2012;2012:10. Available from: http://dx.doi .org/10 . $1155 / 2012 / 489456$.

[85] Schenk D, Barbour R, Dunn W, Gordon G, Grajeda H, Guido T, et al. Immunization with amyloid-[beta] attenuates Alzheimer-disease-like pathology in the PDAPP mouse [Journal Article]. Nature. 1999;400(6740):173-177. Available from: http: //dx.doi.org/10.1038/22124. 
[86] Brazil MI, Chung H, Maxfield FR. Effects of Incorporation of Immunoglobulin G and Complement Component Clq on Uptake and Degradation of Alzheimer's Disease Amyloid Fibrils by Microglia [Journal Article]. Journal of Biological Chemistry. 2000;275(22):16941-16947. Available from: http://www.jbc.org/ content/275/22/16941. abstract.

[87] Bacskai BJ, Kajdasz ST, Christie RH, Carter C, Games D, Seubert P, et al. Imaging of amyloid- $\beta$ deposits in brains of living mice permits direct observation of clearance of plaques with immunotherapy [Journal Article]. Nature medicine. 2001;7(3):369-372.

[88] Bard F, Barbour R, Cannon C, Carretto R, Fox M, Games D, et al. Epitope and isotype specificities of antibodies to $\beta$-amyloid peptide for protection against Alzheimer's disease-like neuropathology [Journal Article]. Proceedings of the National Academy of Sciences. 2003;100(4):2023-2028. Available from: http: //www . pnas.org/content/100/4/2023. abstract.

[89] Wilcock DM, DiCarlo G, Henderson D, Jackson J, Clarke K, Ugen KE, et al. Intracranially Administered Anti-A $\beta$ Antibodies Reduce $\beta$-Amyloid Deposition by Mechanisms Both Independent of and Associated with Microglial Activation [Journal Article]. The Journal of Neuroscience. 2003;23(9):3745-3751. Available from: http: //www. jneurosci.org/content/23/9/3745. abstract.

[90] Zotova E, Holmes C, Johnston D, Neal JW, Nicoll JAR, Boche D. Microglial alterations in human Alzheimer's disease following A $\beta 42$ immunization [Journal Article]. Neuropathology and Applied Neurobiology. 2011;37(5):513-524. Available from: http://dx.doi.org/10.1111/j.1365-2990.2010.01156.x.

[91] Wilcock DM, Munireddy SK, Rosenthal A, Ugen KE, Gordon MN, Morgan D. Microglial activation facilitates $A \beta$ plaque removal following intracranial anti-A $\beta$ antibody administration [Journal Article]. Neurobiology of disease. 2004;15(1):1120. Available from: http://www.sciencedirect.com/science/article/pii/ S0969996103001955.

[92] Wilcock DM, Rojiani A, Rosenthal A, Levkowitz G, Subbarao S, Alamed J, et al. Passive Amyloid Immunotherapy Clears Amyloid and Transiently Activates Microglia in a Transgenic Mouse Model of Amyloid Deposition [Journal Article]. The Journal of Neuroscience. 2004;24(27):6144-6151. Available from: http:// www . jneurosci . org/content/24/27/6144. abstract.

[93] DeMattos RB, Bales KR, Cummins DJ, Dodart JC, Paul SM, Holtzman DM. Peripheral anti-A $\beta$ antibody alters CNS and plasma $A \beta$ clearance and decreases brain $A \beta$ burden in a mouse model of Alzheimer's disease [Journal Article]. Proceedings of the National Academy of Sciences. 2001;98(15):8850-8855. Available from: http: //www . pnas . org/content/98/15/8850 . abstract.

[94] DeMattos RB, Bales KR, Cummins DJ, Paul SM, Holtzman DM. Brain to Plasma Amyloid- $\beta$ Efflux: a Measure of Brain Amyloid Burden in a Mouse 
Model of Alzheimer's Disease [Journal Article]. Science. 2002;295(5563):22642267. Available from: http://www.sciencemag.org/content/295/5563/ 2264. abstract.

[95] Samadi H, Sultzer D. Solanezumab for Alzheimer's disease [Journal Article]. Expert Opinion on Biological Therapy. 2011;11(6):787-798. Available from: http://dx . doi .org/10.1517/14712598.2011.578573.

[96] Seubert P, Barbour R, Khan K, Motter R, Tang P, Kholodenko D, et al. Antibody Capture of Soluble A $\beta$ Does Not Reduce Cortical A $\beta$ Amyloidosis in the PDAPP Mouse [Journal Article]. Neurodegenerative Diseases. 2008;5(2):65-71. Available from: http: //www.karger.com/DOI/10.1159/000112834.

[97] Levites Y, Smithson LA, Price RW, Dakin RS, Yuan B, Sierks MR, et al. Insights into the mechanisms of action of anti-A $\beta$ antibodies in Alzheimer's disease mouse models [Journal Article]. The FASEB Journal. 2006;20(14):2576-2578. Available from: http://www. fasebj.org/content/20/14/2576. abstract.

[98] Dodart JC, Bales KR, Gannon KS, Greene SJ, DeMattos RB, Mathis C, et al. Immunization reverses memory deficits without reducing brain A[beta] burden in Alzheimer's disease model [Journal Article]. Nat Neurosci. 2002;5(5):452-457. Available from: http: //dx. doi.org/10.1038/nn842.

[99] Deane R, Du Yan S, Submamaryan RK, LaRue B, Jovanovic S, Hogg E, et al. RAGE mediates amyloid-[beta] peptide transport across the blood-brain barrier and accumulation in brain [Journal Article]. Nat Med. 2003;9(7):907-913. Available from: http://dx.doi.org/10.1038/nm890.

[100] Bachmeier CJ, Beaulieu-Abdelahad D, Mullan MJ, Paris D. Epitope-Dependent Effects of Beta-Amyloid Antibodies on Beta-Amyloid Clearance in an In Vitro Model of the Blood-Brain Barrier [Journal Article]. Microcirculation. 2011;18(5):373-379. Available from: http://dx.doi.org/10.1111/j.1549-8719.2011.00096.x.

[101] Henderson SJ, Andersson C, Narwal R, Janson J, Goldschmidt TJ, Appelkvist P, et al. Sustained peripheral depletion of amyloid- $\beta$ with a novel form of neprilysin does not affect central levels of amyloid- $\beta$ [Journal Article]. Brain. 2014;137(2):553564. Available from: http://brain. oxfordjournals .org/brain/137/2/553. full.pdf.

[102] Yamada K, Yabuki C, Seubert P, Schenk D, Hori Y, Ohtsuki S, et al. A $\beta$ immunotherapy: intracerebral sequestration of $A \beta$ by an anti-A $\beta$ monoclonal antibody 266 with high affinity to soluble A $\beta$ [Journal Article]. The Journal of Neuroscience. 2009;29(36):11393-11398.

[103] Deane R, Sagare A, Hamm K, Parisi M, LaRue B, Guo H, et al. IgG-Assisted Age-Dependent Clearance of Alzheimer's Amyloid $\beta$ Peptide by the Blood-Brain Barrier Neonatal Fc Receptor [Journal Article]. The Journal of Neuroscience. 2005;25(50):11495-11503. 
[104] Du Y, Wei X, Dodel R, Sommer N, Hampel H, Gao F, et al. Human anti- $\beta$-amyloid antibodies block $\beta$-amyloid fibril formation and prevent $\beta$-amyloid-induced neurotoxicity [Journal Article]. Brain. 2003;126(9):1935-1939. Available from: http: //brain . oxfordjournals . org/content/126/9/1935. abstract.

[105] Frenkel D, Balass M, Katchalski-Katzir E, Solomon B. High affinity binding of monoclonal antibodies to the sequential epitope EFRH of $\beta$-amyloid peptide is essential for modulation of fibrillar aggregation [Journal Article]. Journal of neuroimmunology. 1999;95(1-2):136-142. Available from: http://www . sciencedirect.com/science/article/pii/S016557289900003X.

[106] Mamikonyan G, Necula M, Mkrtichyan M, Ghochikyan A, Petrushina I, Movsesyan N, et al. Anti-A $\beta$ 1-11 Antibody Binds to Different $\beta$-Amyloid Species, Inhibits Fibril Formation, and Disaggregates Preformed Fibrils but Not the Most Toxic Oligomers [Journal Article]. Journal of Biological Chemistry. 2007;282(31):22376-22386. Available from: http: //www. jbc.org/content/282/31/22376. abstract.

[107] McLaurin J, Cecal R, Kierstead M, Tian X, Phinney AL, Manea M, et al. Therapeutically effective antibodies against amyloid- $\beta$ peptide target amyloid- $\beta$ residues 4-10 and inhibit cytotoxicity and fibrillogenesis [Journal Article]. Nature medicine. 2002;8(11):1263-1269.

[108] Solomon B, Koppel R, Hanan E, Katzav T. Monoclonal antibodies inhibit in vitro fibrillar aggregation of the Alzheimer beta-amyloid peptide [Journal Article]. Proceedings of the National Academy of Sciences. 1996;93(1):452-455. Available from: http://www.pnas .org/content/93/1/452. abstract.

[109] Solomon B, Koppel R, Frankel D, Hanan-Aharon E. Disaggregation of Alzheimer $\beta$-amyloid by site-directed mAb [Journal Article]. Proceedings of the National Academy of Sciences. 1997;94(8):4109-4112. Available from: http: //www . pnas . org/content/94/8/4109. abstract.

[110] Bacskai BJ, Kajdasz ST, McLellan ME, Games D, Seubert P, Schenk D, et al. NonFc-Mediated Mechanisms Are Involved in Clearance of Amyloid- $\beta$ In Vivo by Immunotherapy [Journal Article]. The Journal of Neuroscience. 2002;22(18):78737878. Available from: http://www.jneurosci.org/content/22/18/7873. abstract.

[111] Legleiter J, Czilli DL, Gitter B, DeMattos RB, Holtzman DM, Kowalewski T. Effect of Different Anti-A $\beta$ Antibodies on $A \beta$ Fibrillogenesis as Assessed by Atomic Force Microscopy [Journal Article]. Journal of Molecular Biology. 2004;335(4):9971006. Available from: http://www.sciencedirect.com/science/article/ pii/S0022283603014116.

[112] Zameer A, Kasturirangan S, Emadi S, Nimmagadda SV, Sierks MR. Antioligomeric A $\beta$ Single-chain Variable Domain Antibody Blocks A $\beta$-induced Toxicity Against Human Neuroblastoma Cells [Journal Article]. Journal of Molecular Biology. 2008;384(4):917-928. Available from: http://www.sciencedirect. com/science/article/pii/S0022283608012254. 
[113] Liu R, McAllister C, Lyubchenko Y, Sierks MR. Proteolytic Antibody Light Chains Alter $\beta$-Amyloid Aggregation and Prevent Cytotoxicity [Journal Article]. Biochemistry. 2004;43(31):9999-10007. Available from: http://dx . doi . org/10 . $1021 /$ bi 0492354.

[114] Paul S, Planque S, Nishiyama Y. Immunological origin and functional properties of catalytic autoantibodies to amyloid $\beta$ peptide [Journal Article]. Journal of clinical immunology. 2010;30(1):43-49.

[115] Taguchi H, Planque S, Nishiyama Y, Symersky J, Boivin S, Szabo P, et al. Autoantibody-catalyzed Hydrolysis of Amyloid $\beta$ Peptide [Journal Article]. Journal of Biological Chemistry. 2008;283(8):4714-4722. Available from: http://www . jbc.org/content/283/8/4714. abstract.

[116] Brasnjevic I, Steinbusch HWM, Schmitz C, Martinez-Martinez P. Delivery of peptide and protein drugs over the blood-brain barrier [Journal Article]. Progress in Neurobiology. 2009;87(4):212-251. Available from: http://www . sciencedirect.com/science/article/pii/S0301008209000124.

[117] Banks WA, Terrell B, Farr SA, Robinson SM, Nonaka N, Morley JE. Passage of amyloid $\beta$ protein antibody across the blood-brain barrier in a mouse model of Alzheimer's disease [Journal Article]. Peptides. 2002;23(12):22232226. Available from: http://www.sciencedirect.com/science/article/ pii/S0196978102002619.

[118] Broadwell RD, Sofroniew MV. Serum Proteins Bypass the Blood-Brain Fluid Barriers for Extracellular Entry to the Central Nervous System [Journal Article]. Experimental Neurology. 1993;120(2):245-263. Available from: http://www . sciencedirect.com/science/article/pii/S0014488683710599.

[119] Cooper PR, Ciambrone GJ, Kliwinski CM, Maze E, Johnson L, Li Q, et al. Efflux of monoclonal antibodies from rat brain by neonatal Fc receptor, FcRn [Journal Article]. Brain Research. 2013;1534:13-21. Available from: http://www . sciencedirect.com/science/article/pii/S0006899313011724.

[120] Abuqayyas L, Balthasar JP. Investigation of the role of FcR and FcRn in $\mathrm{mAb}$ distribution to the brain [Journal Article]. Molecular pharmaceutics. 2012;10(5):1505-1513.

[121] Garg A, Balthasar J. Investigation of the Influence of FcRn on the Distribution of IgG to the Brain [Journal Article]. The AAPS Journal. 2009;11(3):553-557. Available from: http://dx.doi.org/10.1208/s12248-009-9129-9.

[122] Farrall AJ, Wardlaw JM. Blood-brain barrier: Ageing and microvascular disease - systematic review and meta-analysis [Journal Article]. Neurobiology of Aging. 2009;30(3):337-352. Available from: http://www.sciencedirect.com/ science/article/pii/S0197458007002904. 
[123] Biron KE, Dickstein DL, Gopaul R, Jefferies WA. Amyloid Triggers Extensive Cerebral Angiogenesis Causing Blood Brain Barrier Permeability and Hypervascularity in Alzheimer's Disease [Journal Article]. PLoS One. 2011;6(8):e23789. Available from: http://dx.doi.org/10.1371\%2Fjournal.pone.0023789.

[124] Jaeger LB, Dohgu S, Sultana R, Lynch JL, Owen JB, Erickson MA, et al. Lipopolysaccharide alters the blood-brain barrier transport of amyloid $\beta$ protein: A mechanism for inflammation in the progression of Alzheimer's disease [Journal Article]. Brain, Behavior, and Immunity. 2009;23(4):507517. Available from: http://www.sciencedirect.com/science/article/ pii/S0889159109000440.

[125] Dickstein DL, Biron KE, Ujiie M, Pfeifer CG, Jeffries AR, Jefferies WA. A $\beta$ peptide immunization restores blood-brain barrier integrity in Alzheimer disease [Journal Article]. The FASEB Journal. 2006;20(3):426-433. Available from: http://www . fasebj.org/content/20/3/426. abstract.

[126] Poduslo JF, Curran GL, Berg CT. Macromolecular permeability across the bloodnerve and blood-brain barriers [Journal Article]. Proceedings of the National Academy of Sciences of the United States of America. 1994;91(12):5705-5709. Available from: http://www.ncbi.nlm.nih.gov/pmc/articles/PMC44065/.

[127] Senior K. Dosing in phase II trial of Alzheimer's vaccine suspended [Journal Article]. The Lancet Neurology. 2002;1(1):3. Available from: http://www . sciencedirect.com/science/article/pii/S1474442202000236.

[128] Ragupathi G, Gardner JR, Livingston PO, Gin DY. Natural and synthetic saponin adjuvant QS-21 for vaccines against cancer [Journal Article]. Expert review of vaccines. 2011;10(4):463-470.

[129] Hock C, Konietzko U, Streffer JR, Tracy J, Signorell A, Moller-Tillmanns B, et al. Antibodies against $\beta$-Amyloid Slow Cognitive Decline in Alzheimer's Disease [Journal Article]. Neuron. 2003;38(4):547-554. Available from: http://www . sciencedirect.com/science/article/pii/S0896627303002940.

[130] Holmes C, Boche D, Wilkinson D, Yadegarfar G, Hopkins V, Bayer A, et al. Long-term effects of A 442 immunisation in Alzheimer's disease: follow-up of a randomised, placebo-controlled phase I trial [Journal Article]. The Lancet. 2008;372(9634):216-223. Available from: http://www.sciencedirect.com/ science/article/pii/S0140673608610752.

[131] Vellas B, Black R, Thal LJ, Fox NC, Daniels M, McLennan G, et al. LongTerm Follow-Up of Patients Immunized with AN1792: Reduced Functional Decline in Antibody Responders [Journal Article]. Current Alzheimer Research. 2009;6(2):144-151. Available from: http://www.ncbi.nlm.nih.gov/pmc/ articles/PMC2825665/.

[132] Lee M, Bard F, Johnson-Wood K, Lee C, Hu K, Griffith SG, et al. A $\beta 42$ immunization in Alzheimer's disease generates $\mathrm{A} \beta \mathrm{N}$-terminal antibodies [Journal Article]. 
Annals of Neurology. 2005;58(3):430-435. Available from: http://dx.doi.org/ 10.1002/ana. 20592.

[133] Sergeant N, Bombois S, Ghestem A, Drobecq H, Kostanjevecki V, Missiaen C, et al. Truncated beta-amyloid peptide species in pre-clinical Alzheimer's disease as new targets for the vaccination approach [Journal Article]. Journal of Neurochemistry. 2003;85(6):1581-1591. Available from: http://dx.doi.org/ $10.1046 / j .1471-4159.2003 .01818 . x$.

[134] Boche D, Zotova E, Weller RO, Love S, Neal JW, Pickering RM, et al. Consequence of $A \beta$ immunization on the vasculature of human Alzheimer's disease brain [Journal Article]. Brain. 2008;131(12):3299-3310. Available from: http://brain. oxfordjournals .org/brain/131/12/3299.full.pdf.

[135] Patton RL, Kalback WM, Esh CL, Kokjohn TA, Van Vickle GD, Luehrs DC, et al. Amyloid- $\beta$ Peptide Remnants in AN-1792-Immunized Alzheimer's Disease Patients: A Biochemical Analysis [Journal Article]. The American Journal of Pathology. 2006;169(3):1048-1063. Available from: http://www . sciencedirect.com/science/article/pii/S0002944010627794.

[136] Boche D, Donald J, Love S, Harris S, Neal JW, Holmes C, et al. Reduction of aggregated Tau in neuronal processes but not in the cell bodies after $A \beta 42$ immunisation in Alzheimer's disease [Journal Article]. Acta Neuropathologica. 2010;120(1):1320. Available from: http://dx.doi.org/10.1007/s00401-010-0705-y.

[137] Fox NC, Black RS, Gilman S, Rossor MN, Griffith SG, Jenkins L, et al. Effects of $A \beta$ immunization (AN1792) on MRI measures of cerebral volume in Alzheimer disease [Journal Article]. Neurology. 2005;64(9):1563-1572. Available from: http: //www . neurology . org/content/64/9/1563. abstract.

[138] Paquet C, Amin J, Mouton-Liger F, Nasser M, Love S, Gray F, et al. Effect of active A $\beta$ immunotherapy on neurons in human Alzheimer's disease [Journal Article]. The Journal of Pathology. 2015;235(5):721-730. Available from: http://dx . doi .org/ $10.1002 /$ path. 4491.

[139] Nicoll JA, Wilkinson D, Holmes C, Steart P, Markham H, Weller RO. Neuropathology of human Alzheimer disease after immunization with amyloid- $\beta$ peptide: a case report [Journal Article]. Nature medicine. 2003;9(4):448-452.

[140] Cribbs DH, Ghochikyan A, Vasilevko V, Tran M, Petrushina I, Sadzikava N, et al. Adjuvant-dependent modulation of $\mathrm{Th}(1)$ and $\mathrm{Th}(2)$ responses to immunization with $\beta$-amyloid [Journal Article]. International immunology. 2003;15(4):505-514. Available from: http://www.ncbi.nlm.nih.gov/pmc/ articles/PMC1483061/.

[141] Wiessner C, Wiederhold KH, Tissot AC, Frey P, Danner S, Jacobson LH, et al. The second-generation active $\mathrm{A} \beta$ immunotherapy $\mathrm{CAD} 106$ reduces amyloid accumulation in APP transgenic mice while minimizing potential side effects [Journal Article]. The Journal of Neuroscience. 2011;31(25):9323-9331. 
[142] Farlow MR, Andreasen N, Riviere ME, Vostiar I, Vitaliti A, Sovago J, et al. Longterm treatment with active $\mathrm{A} \beta$ immunotherapy with CAD106 in mild Alzheimer's disease [Journal Article]. Alzheimer's Research \& Therapy. 2015;7(1):23. Available from: http://www.ncbi.nlm.nih.gov/pmc/articles/PMC4410460/.

[143] Pasquier F, Sadowsky C, Holstein A, Leterme GLP, Peng Y, Jackson N, et al. Two Phase 2 Multiple Ascending-Dose Studies of Vanutide Cridificar (ACC-001) and QS-21 Adjuvant in Mild-to-Moderate Alzheimer's Disease [Journal Article]. Journal of Alzheimer's Disease. 2016;(Preprint):1-13.

[144] Schneeberger A, Mandler M, Otava O, Zauner W, Mattner F, Schmidt W. Development of affitope vaccines for Alzheimer's disease (AD)-from concept to clinical testing [Journal Article]. JNHA-The Journal of Nutrition, Health and Aging. 2009;13(3):264-267.

[145] Schneeberger A, Hendrix S, Ellison N, Börger V, Dubois B. Additional results from a phase ii study to assess the clinical and immunological activity, safety, and tolerability of affitope-ad02 in patients with early Alzheimer's disease (AD) [Journal Article]. Alzheimer's \& Dementia: The Journal of the Alzheimer's Association. 2015;11(7):P276.

[146] Muhs A, Hickman DT, Pihlgren M, Chuard N, Giriens V, Meerschman C, et al. Liposomal vaccines with conformation-specific amyloid peptide antigens define immune response and efficacy in APP transgenic mice [Journal Article]. Proceedings of the National Academy of Sciences. 2007;104(23):9810-9815.

[147] Bouter Y, Noguerola JSL, Tucholla P, Crespi GAN, Parker MW, Wiltfang J, et al. Abeta targets of the biosimilar antibodies of Bapineuzumab, Crenezumab, Solanezumab in comparison to an antibody against N-truncated Abeta in sporadic Alzheimer disease cases and mouse models [Journal Article]. Acta Neuropathologica. 2015;130(5):713-729. Available from: http://dx.doi.org/ $10.1007 / \mathrm{s} 00401-015-1489-\mathrm{x}$.

[148] Bard F, Cannon C, Barbour R, Burke RL, Games D, Grajeda H, et al. Peripherally administered antibodies against amyloid [beta]-peptide enter the central nervous system and reduce pathology in a mouse model of Alzheimer disease [Journal Article]. Nat Med. 2000;6(8):916-919. Available from: http://dx.doi.org/10 . 1038/78682.

[149] Buttini M, Masliah E, Barbour R, Grajeda H, Motter R, Johnson-Wood K, et al. $\beta$-Amyloid Immunotherapy Prevents Synaptic Degeneration in a Mouse Model of Alzheimer's Disease [Journal Article]. The Journal of Neuroscience. 2005;25(40):9096-9101. Available from: http: //www . jneurosci .org/content/ 25/40/9096. abstract.

[150] Black RS, Sperling RA, Safirstein B, Motter RN, Pallay A, Nichols A, et al. A Single Ascending Dose Study of Bapineuzumab in Patients With Alzheimer Disease [Journal Article]. Alzheimer disease and associated disorders. 
2010;24(2):198-203. Available from: http://www.ncbi.nlm.nih.gov/pmc/ articles/PMC3715117/.

[151] Sperling R, Salloway S, Brooks DJ, Tampieri D, Barakos J, Fox NC, et al. Amyloidrelated imaging abnormalities (ARIA) in Alzheimer's disease patients treated with bapineuzumab: A retrospective analysis [Journal Article]. Lancet neurology. 2012;11(3):241-249. Available from: http://www.ncbi.nlm.nih.gov/pmc/ articles/PMC4063417/.

[152] Martinez-Ramirez S, Greenberg S, Viswanathan A. Cerebral microbleeds: overview and implications in cognitive impairment [Journal Article]. Alzheimer's Research \& Therapy. 2014;6(3):33. Available from: http://alzres.com/content/6/3/33.

[153] Salloway S, Sperling R, Gilman S, Fox NC, Blennow K, Raskind M, et al. A phase 2 multiple ascending dose trial of bapineuzumab in mild to moderate Alzheimer disease [Journal Article]. Neurology. 2009;73(24):2061-70. Available from: <GotoISI : //MEDLINE: 19923550.

[154] Rinne JO, Brooks DJ, Rossor MN, Fox NC, Bullock R, Klunk WE, et al. 11 C-PiB PET assessment of change in fibrillar amyloid- $\beta$ load in patients with Alzheimer's disease treated with bapineuzumab: a phase 2, double-blind, placebo-controlled, ascending-dose study [Journal Article]. The Lancet Neurology. 2010;9(4):363-372.

[155] Salloway S, Sperling R, Fox NC, Blennow K, Klunk W, Raskind M, et al. Two Phase 3 Trials of Bapineuzumab in Mild-to-Moderate Alzheimer's Disease [Journal Article]. New England Journal of Medicine. 2014;370(4):322-333. Available from: http://www .nejm.org/doi/full/10.1056/NEJMoa1304839.

[156] Liu E, Schmidt ME, Margolin R, Sperling R, Koeppe R, Mason NS, et al. Amyloid- $\beta$ 11C-PiB-PET imaging results from 2 randomized bapineuzumab phase $3 \mathrm{AD}$ trials [Journal Article]. Neurology. 2015;85(8):692-700. Available from: http://www . neurology . org/content/85/8/692. abstract.

[157] Novak G, Fox N, Clegg S, Nielsen C, Einstein S, Lu Y, et al. Changes in Brain Volume with Bapineuzumab in Mild to Moderate Alzheimer's Disease [Journal Article]. Journal of Alzheimer's Disease. 2015;(Preprint):1-12.

[158] Pfizer, Development JAIR, C LL [Generic]; 2013. Available from: https:// ClinicalTrials.gov/show/NCT01193608.

[159] Pfizer, Development JAIR, C LL [Generic]; 2014. Available from: https:// ClinicalTrials.gov/show/NCT01369225.

[160] Van Der Neut Kolfschoten M, Schuurman J, Losen M, Bleeker WK, MartinezMartinez P, Vermeulen E, et al. Anti-inflammatory activity of human IgG4 antibodies by dynamic Fab arm exchange [Journal Article]. Science. 2007;317(5844):1554-1557.

[161] Bruhns P. Properties of mouse and human IgG receptors and their contribution to disease models [Journal Article]. Blood. 2012;119(24):5640-5649. 
[162] Farlow M, Arnold SE, van Dyck CH, Aisen PS, Snider BJ, Porsteinsson AP, et al. Safety and biomarker effects of solanezumab in patients with Alzheimer's disease [Journal Article]. Alzheimer's \& Dementia. 2012;8(4):261271. Available from: http://www.sciencedirect.com/science/article/ pii/S1552526011029438.

[163] Doody RS, Thomas RG, Farlow M, Iwatsubo T, Vellas B, Joffe S, et al. Phase 3 Trials of Solanezumab for Mild-to-Moderate Alzheimer's Disease [Journal Article]. New England Journal of Medicine. 2014;370(4):311-321. Available from: http: //www . nejm.org/doi/full/10.1056/NEJMoa1312889.

[164] Siemers ER, Sundell KL, Carlson C, Case M, Sethuraman G, Liu-Seifert H, et al. Phase 3 solanezumab trials: Secondary outcomes in mild Alzheimer's disease patients [Journal Article]. Alzheimer's \& Dementia. 2015;Available from: http: //www.sciencedirect.com/science/article/pii/S1552526015021482.

[165] Liu-Seifert H, Siemers E, Holdridge KC, Andersen SW, Lipkovich I, Carlson C, et al. Delayed-start analysis: Mild Alzheimer's disease patients in solanezumab trials, 3.5 years [Journal Article]. Alzheimer's \& Dementia: Translational Research \& Clinical Interventions. 2015;1(2):111-121.

[166] Company EL [Clinical Trial]; 2016. Available from: https://ClinicalTrials . gov/show/NCT01900665.

[167] of Medicine WUS, Company EL, Roche HL, Association A, on Aging NI, Avid, et al.. Dominantly Inherited Alzheimer Network Trial: An Opportunity to Prevent Dementia. A Study of Potential Disease Modifying Treatments in Individuals at Risk for or With a Type of Early Onset Alzheimer's Disease Caused by a Genetic Mutation [Clinical Trial]; 2019. Available from: https://ClinicalTrials.gov/ show/NCT01760005.

[168] Bohrmann B, Baumann K, Benz J, Gerber F, Huber W, Knoflach F, et al. Gantenerumab: a novel human anti-A $\beta$ antibody demonstrates sustained cerebral amyloid- $\beta$ binding and elicits cell-mediated removal of human amyloid$\beta$ [Journal Article]. Journal of Alzheimer's disease : JAD. 2012;28(1):4969. Available from: http://europepmc.org/abstract/MED/21955818http: //dx.doi.org/10.3233/JAD-2011-110977.

[169] Ostrowitzki S, Deptula D, Thurfjell L, et al. MEchanism of amyloid removal in patients with alzheimer disease treated with gantenerumab [Journal Article]. Archives of Neurology. 2012;69(2):198-207. Available from: http: //dx . doi .org/ 10.1001/archneurol.2011.1538.

[170] Reardon S. Alzheimer antibody drugs show questionable potential [Journal Article]. Nat Rev Drug Discov. 2015;14(9):591-592. Available from: http://dx . doi.org/10.1038/nrd4709.

[171] Roche HL [Clinical Trial]; 2018. Available from: https : //ClinicalTrials.gov/ show/NCT02051608. 
[172] Adolfsson O, Pihlgren M, Toni N, Varisco Y, Buccarello AL, Antoniello K, et al. An Effector-Reduced Anti- $\beta$ Amyloid $(A \beta)$ Antibody with Unique $A \beta$ Binding Properties Promotes Neuroprotection and Glial Engulfment of A $\beta$ [Journal Article]. The Journal of Neuroscience. 2012;32(28):9677-9689. Available from: http:// www. jneurosci.org/content/32/28/9677. abstract.

[173] Crespi GAN, Hermans SJ, Parker MW, Miles LA. Molecular basis for mid-region amyloid- $\beta$ capture by leading Alzheimer's disease immunotherapies [Journal Article]. Scientific Reports. 2015;5:9649. Available from: http://dx.doi.org/ 10.1038/srep09649.

[174] Garber K. Genentech's Alzheimer's antibody trial to study disease prevention [Journal Article]. Nat Biotech. 2012;30(8):731-732. Available from: http://dx . doi.org/10.1038/nbt0812-731.

[175] Cummings J, Cho W, Ward M, Friesenhahn M, Brunstein F, Honigberg L, et al. A RANDOMIZED, DOUBLE-BLIND, PLACEBO-CONTROLLED PHASE 2 STUDY TO EVALUATE THE EFFICACY AND SAFETY OF CRENEZUMAB IN PATIENTS WITH MILD TO MODERATE ALZHEIMER'S DISEASE [Journal Article]. Alzheimer's \& Dementia: The Journal of the Alzheimer's Association. 2014;10(4):P275. Available from: http://dx.doi.org/10.1016/j.jalz.2014.04.450.

[176] Bussiere T, Weinreb PH, Engber T, Rhodes K, Arndt J, Qian F, et al.. A method of reducing brain amyloid plaques using anti-ab antibodies [Patent]; 2013.

[177] Ratner M. Biogen's early Alzheimer's data raise hopes, some eyebrows [Journal Article]. Nat Biotech. 2015;33(5):438-438. Available from: http://dx.doi.org/ 10.1038/nbt0515-438.

[178] Biogen. 221AD301 Phase 3 Study of Aducanumab (BIIB037) in Early Alzheimer's Disease [Clinical Trial]; 2020. Available from: https://ClinicalTrials.gov/ show/NCT02477800.

[179] Nilsberth C, Westlind-Danielsson A, Eckman CB, Condron MM, Axelman K, Forsell C, et al. The'Arctic'APP mutation (E693G) causes Alzheimer's disease by enhanced A $\beta$ protofibril formation [Journal Article]. Nature neuroscience. 2001;4(9):887893.

[180] Lai R, Logovinsky V, Kaplow JM, Gu K, Yu Y, Moller C, et al. A firstin-human study of BAN2401, a novel monoclonal antibody against betaamyloid protofibrils [Journal Article]. Alzheimer's \& Dementia. 2013;9(4, Supplement):P689. Available from: http://www.sciencedirect.com/science/ article/pii/S1552526013005098.

[181] Satlin A, Wang J, Logovinsky V, Berry S, Swanson C, Dhadda S, et al. Design of a Bayesian adaptive phase 2 proof-of-concept trial for BAN2401, a putative diseasemodifying monoclonal antibody for the treatment of Alzheimer's disease [Journal Article]. Alzheimer's \& Dementia: Translational Research \& Clinical Interventions. 
2016;2(1):1-12. Available from: http://www.sciencedirect.com/science/ article/pii/S2352873716000032.

[182] Dodel R, Hampel H, Depboylu C, Lin S, Gao F, Schock S, et al. Human antibodies against amyloid $\beta$ peptide: a potential treatment for Alzheimer's disease [Journal Article]. Annals of neurology. 2002;52(2):253-256.

[183] Relkin N. Clinical trials of intravenous immunoglobulin for Alzheimer's disease [Journal Article]. Journal of clinical immunology. 2014;34(1):74-79.

[184] Kile S, Au W, Parise C, Rose K, Donnel T, Hankins A, et al. IVIG treatment of mild cognitive impairment due to Alzheimer's disease: a randomised double-blinded exploratory study of the effect on brain atrophy, cognition and conversion to dementia [Journal Article]. Journal of Neurology, Neurosurgery \& Psychiatry. 2015;Available from: http://jnnp.bmj.com/content/early/ 2015/09/29/jnnp-2015-311486. abstract.

[185] Racke MM, Boone LI, Hepburn DL, Parsadainian M, Bryan MT, Ness DK, et al. Exacerbation of Cerebral Amyloid Angiopathy-Associated Microhemorrhage in Amyloid Precursor Protein Transgenic Mice by Immunotherapy Is Dependent on Antibody Recognition of Deposited Forms of Amyloid $\beta$ [Journal Article]. The Journal of Neuroscience. 2005;25(3):629-636. Available from: http://www . jneurosci.org/content/25/3/629. abstract.

[186] Zago W, Schroeter S, Guido T, Khan K, Seubert P, Yednock T, et al. Vascular alterations in PDAPP mice after anti-A $\beta$ immunotherapy: implications for amyloid-related imaging abnormalities [Journal Article]. Alzheimer's \& Dementia. 2013;9(5):S105-S115.

[187] Arrighi HM, Barakos J, Barkhof F, Tampieri D, Jack C, Melanon D, et al. Amyloid-related imaging abnormalities-haemosiderin (ARIA-H) in patients with Alzheimer's disease treated with bapineuzumab: a historical, prospective secondary analysis [Journal Article]. Journal of Neurology, Neurosurgery \& Psychiatry. 2015;Available from: http://jnnp.bmj.com/content/early/ 2015/02/10/jnnp-2014-309493. abstract.

[188] Wilcock DM, Morgan D, Gordon MN, Taylor TL, Ridnour LA, Wink DA, et al. Activation of matrix metalloproteinases following anti-Ab immunotherapy; implications for microhemorrhage occurrence [Journal Article]. J Neuroinflammation. 2011;8:115.

[189] Benilova I, Karran E, De Strooper B. The toxic A[beta] oligomer and Alzheimer's disease: an emperor in need of clothes [Journal Article]. Nat Neurosci. 2012;15(3):349-357. Available from: http://dx.doi .org/10.1038/nn. 3028.

[190] Maarouf CL, Daugs ID, Kokjohn TA, Kalback WM, Patton RL, Luehrs DC, et al. The biochemical aftermath of anti-amyloid immunotherapy [Journal Article]. Molecular Neurodegeneration. 2010;5(1):1-15. Available from: http://dx.doi . org/10.1186/1750-1326-5-39. 
[191] Nath A, Hall E, Tuzova M, Dobbs M, Jones M, Anderson C, et al. Autoantibodies to amyloid $\beta$-peptide $(A \beta)$ are increased in Alzheimer's disease patients and $A \beta$ antibodies can enhance $A \beta$ neurotoxicity [Journal Article]. NeuroMolecular Medicine. 2003;3(1):29-39. Available from: http://dx.doi .org/10.1385/NMM: $3: 1: 29$.

[192] Morkuniene R, Zvirbliene A, Dalgediene I, Cizas P, Jankeviciute S, Baliutyte G, et al. Antibodies bound to $A \beta$ oligomers potentiate the neurotoxicity of $A \beta$ by activating microglia [Journal Article]. Journal of Neurochemistry. 2013;126(5):604615. Available from: http://dx.doi.org/10.1111/jnc.12332.

[193] Wilcock DM, Zhao Q, Morgan D, Gordon MN, Everhart A, Wilson JG, et al. Diverse Inflammatory Responses in Transgenic Mouse Models of Alzheimer's Disease and the Effect of Immunotherapy on These Responses [Journal Article]. ASN Neuro. 2011;3(5). Available from: http://asn.sagepub.com/content/3/5/ AN20110018. abstract.

[194] Mably AJ, Liu W, Mc Donald JM, Dodart JC, Bard F, Lemere CA, et al. Anti-A $\beta$ antibodies incapable of reducing cerebral $A \beta$ oligomers fail to attenuate spatial reference memory deficits in J20 mice [Journal Article]. Neurobiology of Disease. 2015;82:372-384. Available from: http://www.sciencedirect.com/science/ article/pii/S0969996115300127.

[195] Texidó L, Martin-Satué M, Alberdi E, Solsona C, Matute C. Amyloid $\beta$ peptide oligomers directly activate NMDA receptors [Journal Article]. Cell Calcium. 2011;49(3):184-190. Available from: http://www.sciencedirect. com/science/article/pii/S0143416011000200.

[196] Oddo S, Billings L, Kesslak JP, Cribbs DH, LaFerla FM. A $\beta$ Immunotherapy Leads to Clearance of Early, but Not Late, Hyperphosphorylated Tau Aggregates via the Proteasome [Journal Article]. Neuron. 2004;43(3):321-332. Available from: http: //www.sciencedirect.com/science/article/pii/s0896627304004246.

[197] Rasool S, Martinez-Coria H, Wu JW, LaFerla F, Glabe CG. Systemic vaccination with anti-oligomeric monoclonal antibodies improves cognitive function by reducing $\mathrm{A} \beta$ deposition and tau pathology in 3xTg-AD mice [Journal Article]. Journal of Neurochemistry. 2013;126(4):473-482. Available from: http://dx.doi .org/10 . $1111 /$ jnc. 12305.

[198] Lewczuk P, Mroczko B, Fagan A, Kornhuber J. Biomarkers of Alzheimer's disease and mild cognitive impairment: A current perspective [Journal Article]. Advances in Medical Sciences. 2015;60(1):76-82. Available from: http://www . sciencedirect.com/science/article/pii/S1896112614000972.

[199] Kim J, Eltorai AE, Jiang H, Liao F, Verghese PB, Kim J, et al. Anti-apoE immunotherapy inhibits amyloid accumulation in a transgenic mouse model of $\mathrm{A} \beta$ amyloidosis [Journal Article]. The Journal of experimental medicine. 2012;209(12):2149-2156. 
[200] vom Berg J, Prokop S, Miller KR, Obst J, Kolin RE, Lopategui-Cabezas I, et al. Inhibition of IL-12/IL-23 signaling reduces Alzheimer's disease-like pathology and cognitive decline [Journal Article]. Nature medicine. 2012;18(12):1812-1819.

[201] King DJ. Applications and engineering of monoclonal antibodies. CRC Press; 1998. 



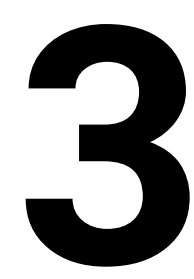

\section{ISOTYPE DEPENDENT EFFECTOR MECHANISMS OF IMMUNOTHERAPY IN AD}

J.A.A. Stevens, A. Franssen, S. Crivelli, P.Martinez-Martinez, M.Losen Submitted 


\section{ABSTRACT}

The anti-amyloid antibody bapineuzumab was recently tested in large clinical trial as a potential treatment for Alzheimer's disease (AD). Unfortunately, the trial did not show a therapeutic effect. In this study, the effect of acute (therapeutic) and preventive treatment of a mouse chimeric version of bapineuzumab (3D6) was investigated in the $5 \mathrm{xFAD}$ mouse model of $\mathrm{AD}$. In addition, to assess the contribution of the inflammatory response, we compared an anti-inflammatory mouse IgG1 (mIgG1) variant of 3D6 with the inflammatory IgG2a (mIgG2a) isotype.

Our results show that treatment with chimeric 3D6 in 5xFAD mice results in passive systemic anaphylaxis (PSA) caused by generation of neutralizing antibodies against the administered 3D6. Furthermore, we show that preventive treatment with both isotypes is able to reduce anxiety-like effects in 5xFAD animals. In addition, mIgG1 worsens memory problems as assessed by the fear conditioning test. Preventive treatment is more efficient in reducing plaques, but causes a shift of $A \beta$ from the formic acid soluble fraction to the detergent soluble fraction. Therapeutic treatment on the other hand, solely causes a reduction of detergent soluble $A \beta$. No treatment regimen had an effect on water soluble A $\beta$. Finally, we show that treatment with mIgG2a 3D6 significantly reduced astrogliosis.

These results show that caution is needed when utilizing immunotherapy for the treatment of $\mathrm{AD}$. In addition, our results show that using isotype specific effects are important to take into consideration and warrant further investigation to further improve immunotherapy for AD. 


\subsection{INTRODUCTION}

Alzheimer's disease (AD) is the main cause of dementia. The best known hallmarks of $\mathrm{AD}$ are the buildup of extracellular plaques consisting mainly out of amyloid- $\beta$ (A $\beta$ ), intracellular tau tangles, neuronal loss and gliosis. In the last decade, several clinical trials utilizing anti-A $\beta$ antibodies have been concluded with largely disappointing results [1].

Only few studies so far have addressed the role of antibody effector mechanisms of immunotherapy in AD. Antibody effector mechanisms are determined by the isotype of the antibody and can involve activation of complement (complement dependent cytotoxicity or CDC), antibody-dependent cellular cytoxocity (ADCC), which is usually mediated by NK cells, or Fc $\gamma$-receptor mediated phagocytosis. For anti-A $\beta$ therapy, CDC and $F c \gamma$-receptor mediated phagocytosis are thought to play the most important role [2]. A third isotype-independent mechanism, called the peripheral sink, is also thought to play a role in $A \beta$ immunotherapy. In this mechanism, anti-A $\beta$ antibodies sequester $A \beta$ in the periphery and prevent them from re-entering the brain, causing a net efflux of $A \beta$ [3].

In this study, isotype dependent effects of the anti-amyloid antibody 3D6 were investigated by recombinant production of mouse IgG1 (mIgG1) and mouse IgG2a (mIgG2a) 3D6. 3D6 is the murine precursor of the humanized antibody bapineuzumab, which has been tested in two large Phase III clinical trials and now has been discontinued because of negative results [4]. Bapineuzumab is recombinantly produced as a human IgG1 (hIgG1) isotype, while the original hybridoma cell-line produces 3D6 as a mouse IgG2a (mIgG2a) isotype. Both isotypes have similar capacities to activate complement and bind Fc-receptors [4]. In humans, IgG4 (hIgG4) antibodies have been shown to lack complement activation capabilities, while also having reduced affinity for Fc $\gamma$-receptors. The mIgG1 isotype is comparable to hIgG4, in the regard that it can only weakly activate complement and has reduced affinity for Fc-receptors [4]. At the moment, an IgG4 variant of bapineuzumab is also being tested in patients with mild to moderate $\mathrm{AD}[5]$.

One reason anti-amyloid trials have been negative so far, might be because treatment started too late [6]. Cross-sectional amyloid imaging studies in patients with familial forms of $\mathrm{AD}$, have shown that $\mathrm{A} \beta$ accumulation starts already up to 15 years before the onset of clinical symptoms [7]. For this reason, several anti-amyloid immunotherapies are now being tested in preventive trials in patients with hereditary forms of AD [8].

In this study, we investigated the effects of immunotherapy with 3D6 using two different isotypes (mIgG1 and mIgG2a). Additionally, efficacy was investigated in a preventive and therapeutic regimen. To this end, we utilized the 5xFAD mouse model of AD. The 5xFAD model contains human APP and PSEN1 with three mutations in APP (APPSwedish, APPFlorida, APPLondon) and 2 in PSEN1 (PSEN1*M146L*L286V), with the first plaques appearing at $\sim 3$ months of age, and cognitive deficits first apparent at 5 months of age. 


\subsection{METHODS}

\subsubsection{ANIMAL BREEDING}

A breeding colony was established of B6/SJL (WT) and 5xFAD animals that were obtained from Jackson Laboratories (stock 6554). Mice were bred hemizygously as described, except that F3 B6/SJL mice were used for generating experimental groups. Additionally, the Pde6b gene was absent in our colony. All mice were female, housed socially on a reversed 12 hour day-night cycle and had ad libitum access to food and water. This experiment was approved by the Animal Welfare Committee at Maastricht University (DEC2013-006) and followed the laws and regulations of the Netherlands.

\subsubsection{ANTIBODY PRODUCTION}

Bapineuzumab variable heavy $(\mathrm{VH})$ and variable light $(\mathrm{VL})$ sequences (humanized sequences) were synthesized by GeneArt (Invitrogen). The $\mathrm{CH} 1, \mathrm{CH} 2$ and $\mathrm{CH} 3$ sequences of the constant heavy chain of mIgG1, mIgG2a and CL mouse kappa light chain sequences were cloned from pFuse plasmids (Invivogen) into a custom designed vector containing a CMV5 promotor and a WPRE element. Additionally, the mIgG1 sequence was mutated in the $\mathrm{Fc} \gamma$-receptor binding site with E233P and in the C1q binding site with E318A, K320A, and R322A to completely eliminate binding to the Fc $\gamma$-receptor and complement activation $[9,10]$. Clones were verified by sequencing (GATC). Plasmids were produced large scale in a BioFlo 110 fermentor (New Brunswick). Transfected E. coli was grown to high density and plasmid growth was temperature induced. After 2 days, bacteria were pelleted by centrifugation and pellets were frozen at $-80^{\circ} \mathrm{C}$ until further processing.

For purification of plasmids, typically around 8 grams of bacterial pellet was lysed using $150 \mathrm{ml}$ of each buffer of the alkaline lysis method [11]. RNA was precipitated by addition of $\mathrm{CaCl}_{2}$ to a final concentration of $1.4 \mathrm{M}$. Plasmids were subsequently purified on an AKTA explorer system using a CIM DEAE column (BIA Separations).

Antibodies were produced by transient transfection of HEK293F cells. HEK293F cells were grown to confluence in 4-layer cell factories (Nunc) in Freestyle 293 medium. Cells were transfected with $22 \mathrm{kDa}$ linear PEI (Polysciences) with a DNA:PEI ratio of 1:4. Heavy to light chain ratio was 1.5:2. Four hours after transfection, valproic acid was added to a final concentration of $4 \mathrm{mM}$. Cultures were typically maintained for 10-12 days after which the supernatant was further processed.

Supernatant was filtered first through glass wool, then through a glass fiber membrane of $0.8 \mu \mathrm{m}$ and finally through a $0.45 \mu \mathrm{m}$ sterile filter (Pall). Next, supernatant was concentrated on an artificial kidney (Fresenius Medical Care) and purified using a MabSelect protein A column (GE Healthcare) (for mIgG2a) or a custom packed protein G column (for mIgG1) (GE Healthcare) on an AKTA explorer system (GE Healthcare). Eluate was immediately neutralized by means of a buffer exchange to PBS using a column packed with Sephadex G50. Antibody purity was confirmed by electrophoresis and Coomassie blue staining. After purification, antibodies were concentrated using a spin column (Millipore) to a final concentration of $1 \mathrm{mg} / \mathrm{ml}$, sterile filtered by a $0.22 \mu \mathrm{m}$ syringe filter, and stored at $4{ }^{\circ} \mathrm{C}$ until further use. 


\subsubsection{EXPERIMENTAL SETUP AND TISSUE PROCESSING}

The complete experimental setup consisted of 6 groups, of 24 animals each. The experimental setup is depicted in Figure 1. Animals in the preventive regimen received weekly $100 \mu \mathrm{g}$ of mIgG1 3D6 or mIgG2a 3D6 starting at 4 months of age. After 4 weeks, the antibody dose was lowered to $50 \mu \mathrm{g}$. From this point forward, they also received 1 $\mathrm{ml}$ of saline injections subcutaneously in addition to the antibody to reduce side effects caused by antibody administration. All the other groups received weekly PBS injections starting at 4 months of age. At 5 months of age, the animals in the therapeutic groups received $100 \mu \mathrm{g}$ of mIgG1 3D6 or mIgG2a 3D6 per week instead of PBS. During the last two weeks of therapy, animals underwent behavioral testing.

After the behavioral procedures, animals were anaesthetized and perfused. Serum was taken before perfusion and stored at $-80{ }^{\circ} \mathrm{C}$ until further use. One group of animals was perfused using ice-cold Tyrode solution, and brains were snap-frozen with liquid nitrogen. Another group of animals was perfused with ice-cold Tyrode solution followed by fixation with $4 \%$ paraformaldehyde. Brains were post fixated overnight in $4 \%$ paraformaldehyde, after which they were cryoprotected using $20 \%$ sucrose and frozen using $\mathrm{CO}_{2}$. Sagittal brain section with a thickness of $16 \mu \mathrm{m}$ were cut on a cryostat (Leika CM3050S).

For amyloid beta measurements using ELISA, non-fixated frozen brain hemispheres were crushed using a pestle with liquid nitrogen and divided into $10 \mathrm{mg}$ aliquots. For extraction of amyloid, 15 times (w/v) of TBS containing $1 \times$ Complete protease inhibitors (Roche) and phosoSTOP (Roche) was added. Suspensions were homogenized using a micropestle. Following sonication, suspensions were centrifuged for 30 minutes at $30000 \mathrm{~g}$. Supernatant was stored at $-80{ }^{\circ} \mathrm{C}$ (soluble fraction) until further use and an equal volume of TBS containing $1 \%$ Triton was added to the pellets. Pellets were resuspended using a micropestle, sonicated and centrifuged again for 30 minutes at $30000 \mathrm{~g}$. Supernatant was stored at $-80^{\circ} \mathrm{C}$ (insoluble fraction) and pellet was further resuspended to a concentration of $150 \mathrm{mg} / \mathrm{ml}$ in $70 \%$ formic acid. Samples were incubated under gentle agitation for 2 hours at room temperature and finally centrifuged at $30000 \mathrm{~g}$ for 30 minutes. Supernatant was neutralized by addition of 20 times $1 \mathrm{M}$ Tris.

\subsubsection{BEHAVIORAL PROCEDURES}

\section{OPEN FIELD TEST}

The open field test was used to assess anxiety and locomotor changes. The open field consisted of a $50 \times 50 \mathrm{~cm}$ square PVC arena divided into 4 equal fields of $25 \times 25 \mathrm{~cm}$ to test 4 mice in parallel. The arenas were surrounded by grey PVC walls of $25 \mathrm{~cm}$ high. Mice were placed centrally in an arena for 20 minutes and tracked using an overhead attached camera. After each testing round, the arena was thoroughly cleaned with $70 \%$ ethanol. For assessment of locomotor activity, distance moved and velocity were calculated of the entire 20 minutes using Ethovision XT10 (Noldus). As a measure for anxiety, corners and walls were defined as the area up until $8 \mathrm{~cm}$ of distance from the wall, and the time spent in the central area during the first five minutes was measured using Ethovision XT10. 


\section{ELEVATED ZERO MAZE}

To measure anxiety related behavior, the elevated zero maze (EZM) task was used. The EZM consisted of a circular runway with a diameter of $50 \mathrm{~cm}$ and a walking path of 5 $\mathrm{cm}$ wide. It was located $70 \mathrm{~cm}$ above the floor and divided equally into two opposing closed and open parts. The closed parts were surrounded by $50 \mathrm{~cm}$ high walls, while there was a $5 \mathrm{~mm}$ high rim around the walking path of the open part to prevent the mouse from falling off. The mouse was placed centrally into an open arm facing one the closed arms. The EZM was performed in low-light conditions using only red light and animals were tracked using an overhead infrared camera. The EZM was cleaned with $70 \%$ ethanol between tests. For analysis, latency to the first closed arm entry, total distance, and the percentage of time spend in the closed area were measured using Ethovision XT10 (Noldus). The percentage of time spend in the closed area was corrected for the latency until entry into the first closed arm. Two animals were excluded from the analysis because the latency to the first closed arm entry was more than one minute.

\section{ALTERNATING Y-MAZE}

The spontaneous alternating Y-Maze was used to assess spatial working memory. The Y-maze used consisted of 3 acrylic arms arranged at an $120^{\circ}$ angle. The arms were 40 $\mathrm{cm}$ long, $17 \mathrm{~cm}$ long, and with a bottom of $4 \mathrm{~cm}$ wide and an open top of $13 \mathrm{~cm}$ wide. Visual cues were attached to the walls next to maze. Start location was randomized and mice were placed at the end of one of the arms. Mice were left to explore the maze freely for 5 minutes. The maze was thoroughly cleaned with $70 \%$ ethanol between tests. Mice were tracked using an overhead camera and distance moved and percent triads were analyzed automatically using Ethovision XT as described [12]. A triad was defined as a visit through all three arms consecutively. The percentage of alternations was calculated as the number of triads divided by the maximum possible of triads (total number of arm entries minus 2).

\section{CONTEXTUAL AND CUED FEAR CONDITIONING}

Contextual and cued fear conditioning (FC) were used to measure hippocampal and amygdala functioning. The conditioning chamber consisted of a transparent acrylic chamber with metal grid floors. A sound generator was attached directly to the side of the chamber. For cued FC recall, a triangular black plastic wall was placed in the chamber and the floor was covered by a white plastic plate. Between tests of mice, the chamber was cleaned with $70 \%$ ethanol. For conditioning, mice were allowed to acclimatize for 2 minutes after which 4 trials of paired stimuli spaced 1 minute apart were presented. Each trial started with the presentation of a cued conditioning stimulus (CS) consisting of a $70 \mathrm{~dB}$ tone presented for 20 seconds. During the last 2 seconds a foot shock unconditioned stimulus (US) of $0.75 \mathrm{~mA}$ was given. Twenty-four hours after training, contextual freezing behavior was measured in half of the conditioned mice. Mice were recorded continuously with an overhead camera for three minutes in exactly the original chamber and freezing behavior was measured automatically with Ethovision XT10. For the other half of the mice cued freezing behavior was measured. For cued recall, mice were placed in the modified chamber and allowed to acclimatize for 2 minutes. After this period, the CS was presented 4 times for 20 seconds every other minute. Freezing behavior was measured automatically during these 20 seconds using 
Ethovision XT. Forty-eight hours after conditioning, contextual recall was performed for the mice that underwent cued recall the day before and vice versa. Animals that did not show any physical reaction to shocks during the conditioning phase were excluded from the analysis.

\subsubsection{IMMUNOFLUORESCENCE AND HISTOCHEMISTRY}

A triple immunofluorescent staining for microglia, plaques and cell nuclei was performed. Sections were incubated with rabbit anti-Iba-1 (Wako, 1:1000) and human anti-amyloid 20C2 (1:4000) (recombinant anti-amyloid antibody) overnight at $4{ }^{\circ} \mathrm{C}$. Sections were washed with Tris-buffered saline (TBS), TBS with $0.3 \%$ Triton X-100 (TBS-T) and TBS. Next, sections were incubated with mouse anti-human Alexa 594 (Jackson Immuno, 1:500) or donkey anti-rabbit 488 (1:300, Invitrogen). Sections were subsequently washed with TBS, TBS-T, and TBS. Sections were incubated with Hoechst 33342 (Sigma-Aldrich, 1:500) for 15 minutes and washed with three times with TBS. Finally, sections were dried at $50{ }^{\circ} \mathrm{C}$ for one hour, cleared in xylene and cover slipped using DPX (Klinipath). For GFAP staining, the same procedure was followed, except that rabbit anti-GFAP (Dako, 1:1000) was used.

Microhemorrhages were assessed by Prussian blue staining of hemosiderin deposits. Sections were washed in distilled water followed by a 30 -minute incubation in $5 \%$ potassium ferrocyanide (Sigma) with $5 \% \mathrm{HCl}$. Sections were washed 5 times in distilled water, dehydrated, cleared in xylene, and mounted with DPX.

Quantification of GFAP, Ibal and plaques was performed as follows. Two to three midsagittal sections spaced $160 \mu \mathrm{m}$ apart were used per animal. Whole section photomicrographs were acquired using $\mu$ Manager software [13] on a BX51WI spinning disk confocal fluorescence microscope (Olympus) with an EM-CCD C9100 camera (Hamamatsu). Images were background corrected and analyzed using Fiji [14]. For analysis, regions-of-interest (ROIs) were manually delineated. ROIs comprised cortex, subiculum, hippocampus, and midbrain combined with striatum. Next, a median filter was applied, and images were thresholded and converted to binary images. Plaques, microglia, and astrocytes density were measured using the particle analyzer tool. The density was averaged over all sections and this measure was used for statistical analysis. A measure of plaque-associated microglia and astrocytes was obtained by measuring the density of microglia or plaques in a $50 \mu \mathrm{m}^{2}$ radius around the plaques. Densities were averaged per animal and this measure was used for statistical analysis. The same settings were used for all images.

For quantification of microhemorrhages, per animal, 3-4 sections (spaced $160 \mu \mathrm{m}$ ) were analyzed on a BX51 microscope (Olympus). Sections were manually inspected by two investigators who were blind to experimental groups. Incidences were counted per section and averaged per animal.

\subsubsection{ELISA}

For determination of immune-complexes, high-binding ELISA plates (Greiner) were coated with fully human 3D6 or human IgG (Sigma). Plates were blocked with $0.4 \%$ nonfat dry milk (Biorad), followed by incubation with mouse serum (1:10-10 000 diluted). Plates were washed with PBS containing $0.1 \%$ Tween (PBST) and incubated with HRP 
conjugated anti-mouse IgG (Organon, 1:10 000). After washing, plates were developed by incubation with TMB (Sigma) and stopped with $2 \mathrm{M} \mathrm{H}_{2} \mathrm{SO}_{4}$. Possible anti-3D6 mouse IgE was assessed using the same ELISA setup, but using biotinylated anti-mouse IgE (clone RME-1, Biolegend, 1:1000) as detection antibody, followed by incubation with streptavidin-HRP (Jackson Immuno, 1:4000).

ELISA plates were coated with human 266 overnight at room temperature. After washing, plates were blocked with $0.4 \%$ non-fat dry milk and incubated with brain homogenates. Next, plates were washed, incubated with biotinylated 20C2 and washed again. Finally, plates were incubated with streptavidin-HRP (Jackson Immuno) and developed using TMB.

\subsubsection{STATISTICAL ANALYSES}

Statistical analysis was performed using SPSS 23 (IBM) and Graphpad Prism 6. Oneway ANOVA was conducted with a combination of planned contrasts and post-hoc corrections. LSD was used as correction for multiple comparisons. Contrasts were designed to specifically test the following hypotheses: is there a difference between treatment and prevention groups and is there a difference between mIgG1 and mIgG2a groups. 


\subsection{RESULTS}

\subsubsection{IMMUNOTHERAPY WITH IGG1 AND IGG2A 3D6 ANTIBODIES CAUSES INCREASED MORTALITY OF 5XFAD MICE}

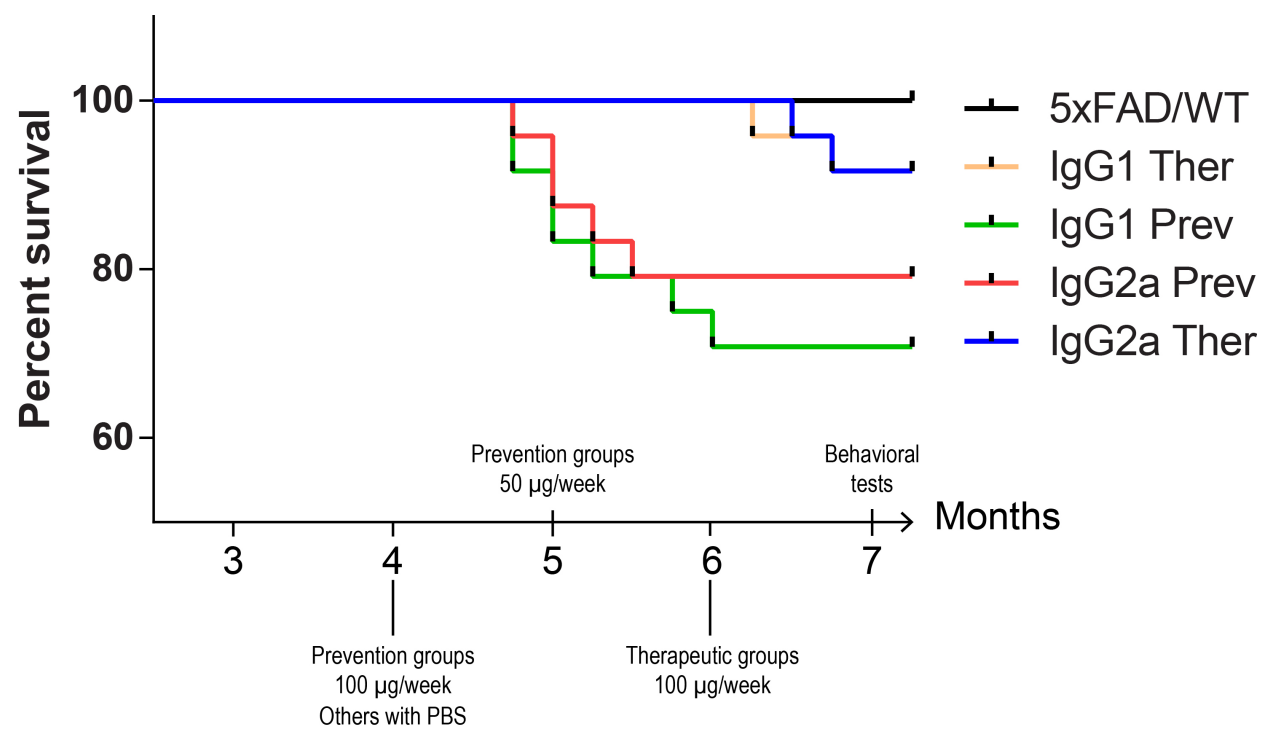

Figure 3.1: A Kaplan-Meyer plot shows that antibody treatment significantly affected survival of animals in experimental groups $(\mathrm{p}=0.04)$. 5xFAD animals were either treated in a preventive or therapeutic fashion with a weekly dose of $100 \mu \mathrm{g}$ of 3D6 per week. The antibody dose in the prevention group was lowered to $50 \mu \mathrm{g} /$ week after 4 weeks because of lethal effects. Control groups (untreated 5xFAD and WT animals) received weekly injections with PBS ( $\mathrm{n}=24$ per group).

5xFAD animals were treated with mIgG1 and mIgG2a 3D6 in either a preventive or therapeutic regimen in an effort to resolve AD symptoms and pathology. Unexpectedly, antibody treatment significantly affected survival of the animals $(p=0.04)$. Nine percent dropout was observed in both antibody groups under the therapeutic regimen. In the preventive mIgG2a group 5 out of 24 animals died ( $20 \%)$, while in the preventive mIgG1 group almost $30 \%$ of animals was lost (7 out of 24) (see Figure 3.1). This difference was not statistically significant $(\mathrm{p}=0.5)$. Compared to the therapeutic group, significantly more animals were lost in the preventive group $(\mathrm{p}=0.03)$. Deaths started occurring after 3 administrations of antibody and always occurred within several hours after injection; in one case, death was observed within half an hour after administration. Symptoms observed after antibody administration were consistent with those of passive systemic anaphylaxis (PSA). Antibody dose in the preventive groups was halved after 4 weeks, which reduced the rate of mice dropping out (see Figure 1)

To exclude that microhemorrhages might have been the cause of death, incidences were counted in brains sections using Prussian blue staining. No significant differences were found between any of the groups $(\mathrm{p}=0.75)$, but average number of microhemorrhages was increased by about $20 \%$ in preventive treated animals compared 
to WT animals (data not shown).

Since the administered antibodies were chimeric antibodies (Fv from human origin and constant region from mouse) a possible immune response against our treatment was assessed in the mouse serum. To this end, fully human 3D6 (bapineuzumab) was coated on Elisa plates and mouse IgG was measured. Additionally, we also measured a possible antibody titer against human IgG.

Figure 3.2A shows that most animals in the immunotherapy groups have a possible anti-3D6 response. Five (19\%) untreated 5xFAD animals also were positive in the anti3D6 ELISA. A possible explanation might be that these animals have natural antibodies against amyloid or against human IgG. In the therapeutic groups $73 \%$ (mIgG1) and $75 \%$ (mIgG2a) of animals were positive, while the percentages in the mIgG1 $(78 \%)$ and mIgG2a (82\%) preventive groups were slightly higher. No animals had IgE antibodies against bapineuzumab (data not shown).

A

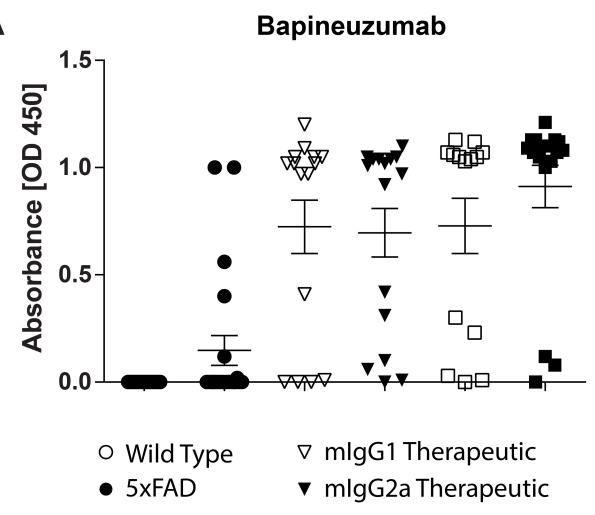

B

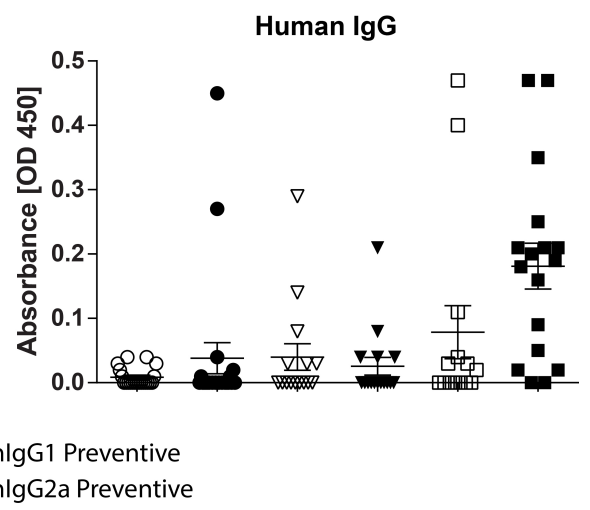

Figure 3.2: A) An ELISA was performed to detect mouse IgG against fully human 3D6 (Bapineuzumab). In groups that received 3D6 $\sim 80 \%$ of animals had possible antibodies against 3D6. B) Mouse antibodies against human IgG were detected by coating with polyclonal human IgG. Preventive treatment with mIgG2a resulted in $70 \%$ of animals developing anti-human IgG antibodies. $\left(\mathrm{n}_{\mathrm{WT}}=21, \mathrm{n}_{5 \mathrm{xFAD}}=21, \mathrm{n}_{\text {mIgG1Ther }}=15, \mathrm{n}_{\mathrm{mIgG}}\right.$ aTher $=16$, $\mathrm{n}_{\text {mIgG1Prev }}=14, \mathrm{n}_{\text {mIgG2aPrev }}=17$ )

To further confirm that these mice developed antibodies against the therapeutic antibody, a mouse anti-human IgG assay was performed. In this assay, 3 untreated 5xFAD animals (10\%) showed a natural cross-reaction to human IgG, while no WT animals were found positive. The therapeutical mIgG1 (20\%), therapeutical mIgG2a (12\%) and preventive mIgG1 group (21\%) all had a low number of positive animals, while in the preventive mIgG2a group $70 \%$ of all animals had anti-human IgG antibodies (see Figure 3.2C).

\subsubsection{MIGG1 WORSENED COGNITIVE DEFICITS IN THE CONTEXTUAL FEAR CONDITIONING TEST}

After antibody treatment, locomotion, anxiety, and memory were assessed using a test battery consisting of the open field test, the alternating Y-maze, the EZM, and the contextual and cued FC test. The open field was used mainly to test locomotion deficits 
in the mice, although anxiety was also assessed by measuring the percentage of time spent in the center of the open field during the first 5 minutes. No difference in the percentage spent in the center could be found between any of the groups $(\mathrm{p}=0.87)$ (see Figure 3.3). However, mice treated therapeutically with mIgG2a showed reduced velocity compared to untreated 5xFAD mice or mice treated in a preventive fashion with mIgG1 $(\mathrm{p}<0.01)$ (see Figure 3.3). Additionally, mice treated with mIgG2a had reduced velocity compared to mice treated with mIgGl $(\mathrm{p}=0.03)$ and also a therapeutic regimen resulted in reduced speed compared to a preventive regimen $(\mathrm{p}=0.049)$.

A

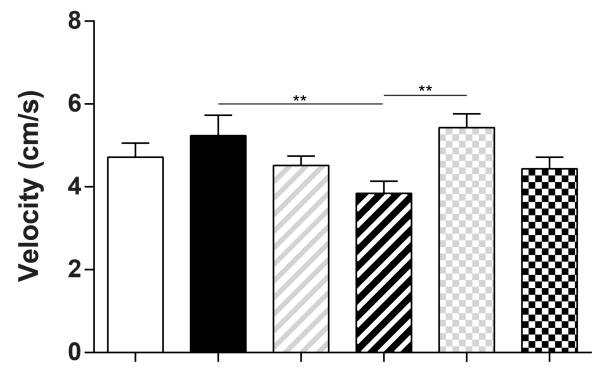

C

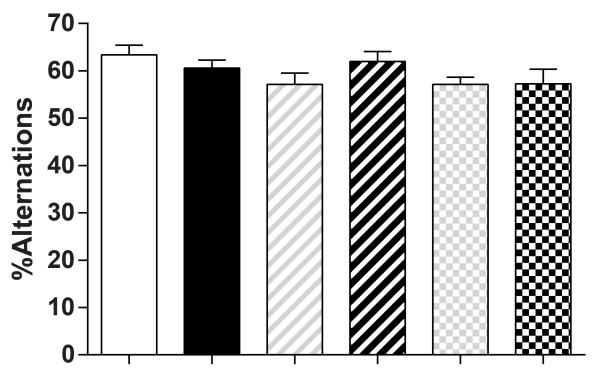

B

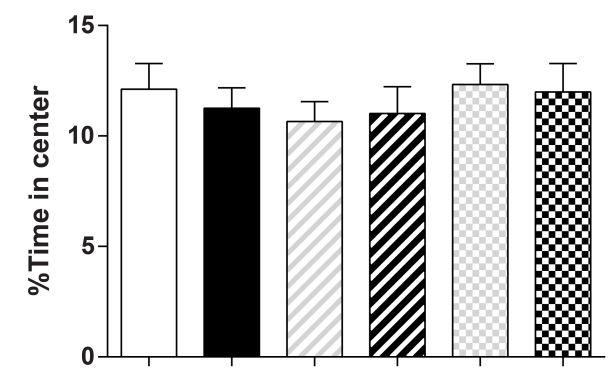

D

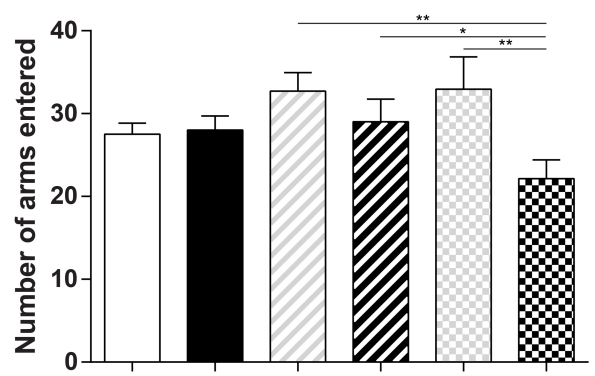

mlgG1 prevention

mlgG2a prevention

Figure 3.3: A) Average velocity in the open field was measured to assess possible locomoter deficits. Velocity was significantly affected by antibody isotype and treatment duration (regimen). Animals treated with mIgG1 animals moved more than mIgG2a and therapeutic animals moving less than preventive animals (B) The time spent in center of the open field was used as a measure for anxiety. No differences were found in the amount of time spent in center $\left(\mathrm{n}_{\mathrm{WT}}=23, \mathrm{n}_{5 \mathrm{xFAD}}=24, \mathrm{n}_{\text {mIgG1Ther }}=18, \mathrm{n}_{\text {mIgG2aThe }} \mathrm{r}=21, \mathrm{n}_{\mathrm{mIgG} \text { Prev }}=16\right.$, $\left.\mathrm{n}_{\text {mIgG2aPrev }}=18\right) \mathrm{C}$-D) The alternating Y-maze was utilized as a test for working memory. (C)No differences were found in the percentage of alternations. (D) An isotype specific effect was present in the number of arms entered, with mIgG2a animals entering less arms than mIgG1 animals $\left(\mathrm{n}_{\mathrm{WT}}=23, \mathrm{n}_{5 \mathrm{xFAD}}=25, \mathrm{n}_{\text {mIgG1Ther }}=20\right.$, $\mathrm{n}_{\text {mIgG2aThe }} \mathrm{r}=22, \mathrm{n}_{\text {mIgG1Prev }}=16, \mathrm{n}_{\text {mIgG2aPrev }}=18$ ) Error bars indicate the SEM in all graphs.

Next, working memory was assessed in the alternating Y-maze. With this test, the working memory of 5-month-old $5 x F A D$ mice was shown to be impaired [15]. We did not observe differences of working memory between the groups $(p=0.29$ ). 
Additionally, when comparing each group to the chance level of $50 \%$, no working memory impairments were found in 5xFAD mice. The mIgG2a prevention group had significantly less arm entries compared to the three other treatment groups (see Figure 3.3). Additionally, both mIgG2a groups had less arm entries compared to the mIgG1 groups $(\mathrm{p}<0.01)$.

The EZM was used to assess anxiety related behavior, which is normally decreased in AD-like animals. Two measures for anxiety were assessed: (1) the latency to enter the closed arm and (2) the percentage of time spent in the closed arms. WT animals entered significantly faster in the closed arm than untreated 5xFAD animals $(\mathrm{p}=0.04)$, or animals in both therapeutic groups ( $\mathrm{p}<0.01$ for both mIgG1 and mIgG2a groups). Animals in the prevention groups performed at a similar level as the WT group and the prevention group performed significant better than the therapeutic group $(\mathrm{p}<0.01)$. No differences were found between any of the groups in the percentage spent in the closed arms.

A

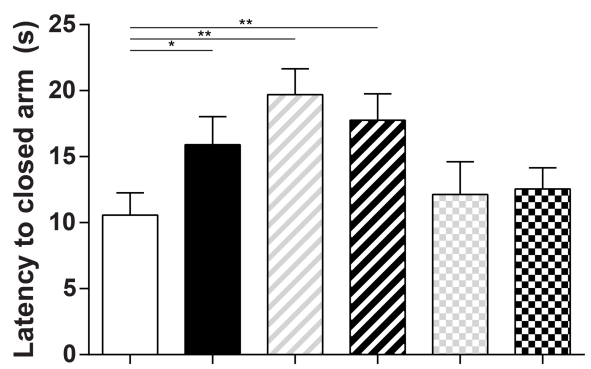

C

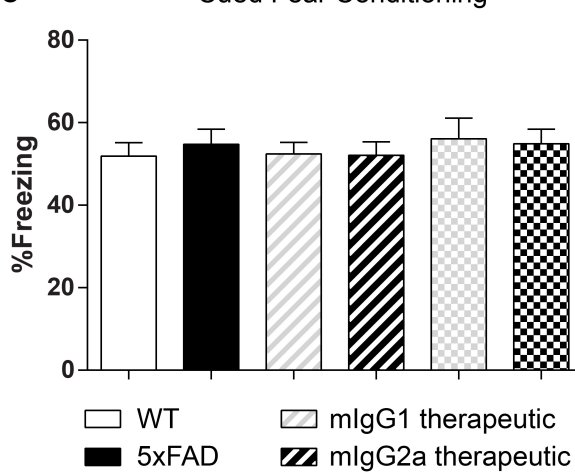

B

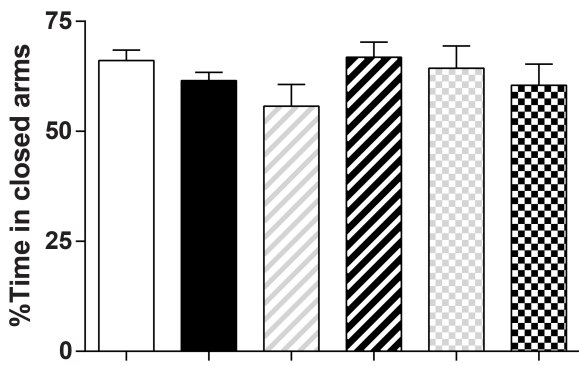

D Contextual Fear Conditioning

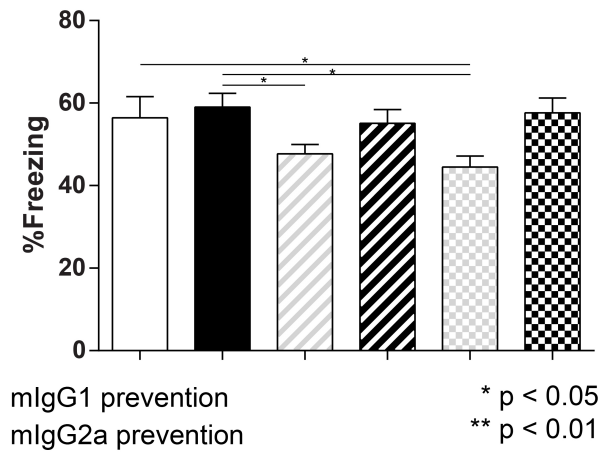

Figure 3.4: A-B) The elevated zero maze (EZM) was used as a measure for anxiety-like behavior A) The latency to enter to closed arm was restored to WT levels in the preventive groups. (B) No differences were found in the time spent in the closed arms $\left(n_{W T}=22, n_{5 \times F A D}=24, n_{\text {mIgG1Ther }}=16, n_{\text {mIgG2aThe }} r=22, n_{\text {mIgG1Prev }}=16\right.$, $\left.\left.\mathrm{n}_{\mathrm{mIgG} 2 \mathrm{aPrev}}=17\right) \mathrm{C}-\mathrm{D}\right)$ The contextual and cued fear conditioning test were used to assess amygdala and hippocampal dependent memory. (C) No differences were present in the cued fear conditioning test. In the contextual fear conditioning test, animals treated with mIgGl performed worse than untreated animals or animals treated with mIgG2a (D) $\left(\mathrm{n}_{\mathrm{WT}}=20, \mathrm{n}_{5 \times \mathrm{XAD}}=19, \mathrm{n}_{\text {mIgG1Ther }}=20, \mathrm{n}_{\text {mIgG2aTher }}=21, \mathrm{n}_{\text {mIgG1Prev }}=13\right.$, $\mathrm{n}_{\text {mIgG2aPrev }}=17$ ) 
While the cued FC test mainly assessed the integrity of the amygdala, the contextual FC test is dependent on integrity of the hippocampus and frontal cortex [16]. In the cued FC test, no difference between any of the groups was observed $(p=0.69)$. However, in the contextual FC test, animals treated with mIgG1 in the therapeutic as well as in the prevention group performed worse than untreated $5 x F A D$ animals $(p=0.01$ and $p=0.02$ for the prevention and therapeutic group respectively). In addition, prevention animals treated with mIgG1 performed worse than WT animals $(\mathrm{p}=0.03)$ and prevention animals treated with mIgG2a $(\mathrm{p}=0.02)$. Overall, there was an effect of antibody isotype, with animals treated with mIgG1 showing less freezing behavior compared to mIgG2a treated animals $(\mathrm{p}<0.01)$.

A

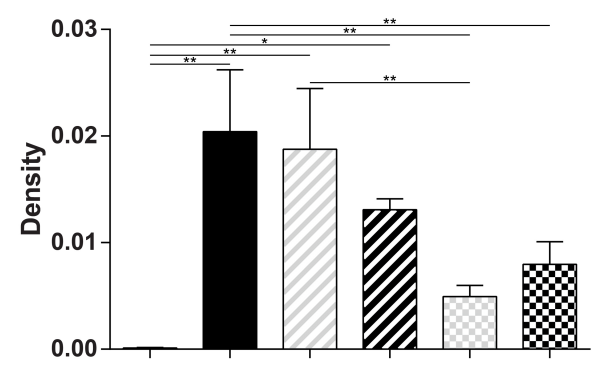

C

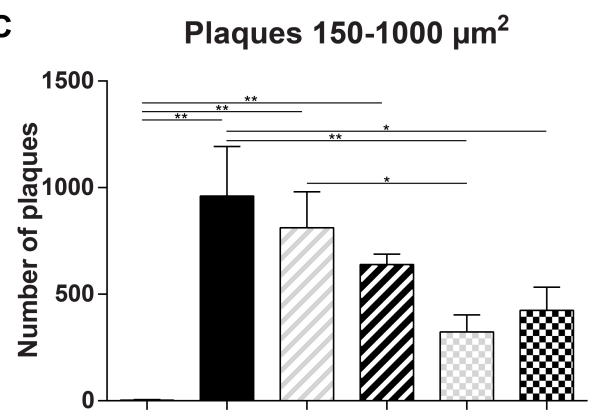

B $\quad$ Plaques $<150 \mu \mathrm{m}^{2}$

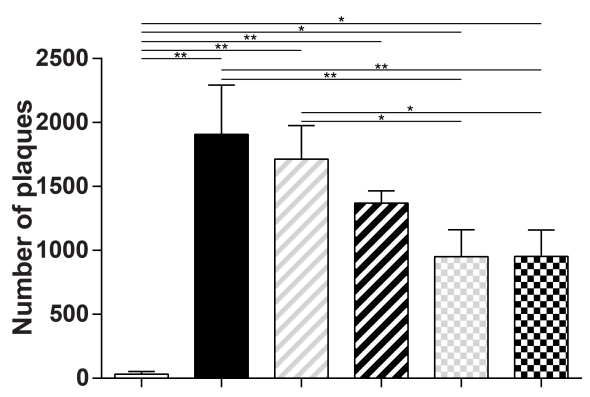

D

Plaques $>1000 \mu \mathrm{m}^{2}$

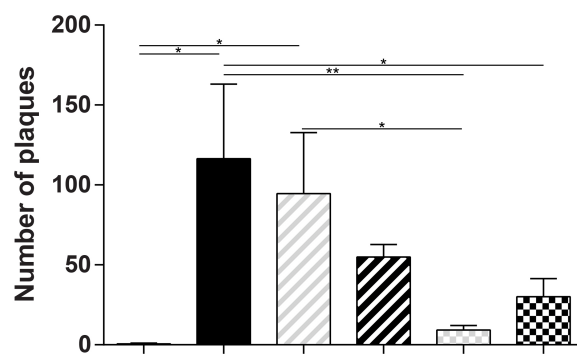

${ }^{*} p<0.05$

${ }^{* *} p<0.01$

Figure 3.5: Sections were stained for $A \beta$ and quantified using ImageJ in the brain (cortex, hippocampus, basal ganglia, thalamus) A) Preventive treatment resulted in a significant reduction of plaque density compared to untreated 5xFAD animals. Therapeutic treatment did not result in a significant reduction of plaque load. BC) Small and medium plaques were reduced by half in the preventive groups compared to untreated 5xFAD animals, while no differences were present with the therapeutic groups. D) Large plaques were reduced five-fold in the preventive groups compared to untreated $5 \times$ FAD animals $\left(n_{\mathrm{WT}}=5, n_{5 \times F A D}=8, n_{\text {mIgGiTher }}=7\right.$, $\mathrm{n}_{\mathrm{mIgG} 2 \mathrm{aTher}}=10, \mathrm{n}_{\mathrm{mIgG1Prev}}=6, \mathrm{n}_{\mathrm{mIgG} 2 \mathrm{aPrev}}=6$ ) Error bars denote $\mathrm{SEM}$. 


\subsubsection{PreVentive immunotherapy Regimens deCREASEd A $\beta$ PLAque LOAD}

Next, the effect of regimen and isotype was investigated on the plaque load and plaque size using immunohistochemistry. Immunotherapy significantly reduced plaque load compared to untreated 5xFAD animals, but only with long term treatment in both prevention groups $(\mathrm{p}=0.01)$. Overall, the preventive mIgG1 group had the strongest reduction in plaque load, followed by the preventive mIgG2a group (see Figure 3.5A). No significant reduction was found in the therapeutic groups when compared to untreated 5xFAD. The preventive mIgG1 group showed a significant lower plaque load compared to the therapeutic mIgG1 group ( $\mathrm{p}=0.01)$, but not with the therapeutic mIgG2a group $(\mathrm{p}=0.12)$. There was no significant difference between untreated 5xFAD animals and therapeutically treated mIgG1 animals $(\mathrm{p}=0.75)$, while a small, but non-significant decrease was observed in the therapeutic mIgG2a group compared to untreated animals $(\mathrm{p}=0.12)$. No isotype specific effects were found $(\mathrm{p}=0.72)$.

The effects of immunotherapy on total plaque load was investigated further by measuring the size of individual plaques. Preventive IgG administration reduced small and medium sized plaques by half compared to untreated animals $(\mathrm{p}=0.02$ and $\mathrm{p}=0.01$ ), while large sized plaques were reduced almost ten-fold $(\mathrm{p}=0.03)$ (see Figure $3.5 \mathrm{~B}$ D). Again, only the preventive immunotherapy regimens reduced plaque numbers of different sizes, compared to untreated 5xFAD mice.
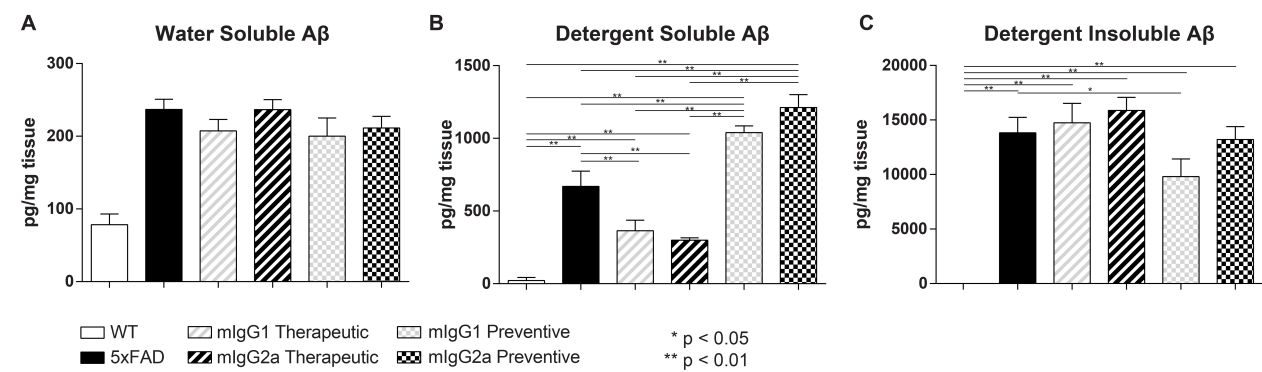

Figure 3.6: $A \beta$ in different fraction (TBS, Triton, Formic acid (FA)) was determined by ELISA in total brain homogenates. A) Immunotherapy did not result in a reduction of soluble $A \beta$ in any of the groups. B) Therapeutic treatment with $3 D 6$ resulted in a significant reduction of triton soluble $A \beta$ compared to untreated $5 x F A D$ animals. In prevention groups, triton soluble $A \beta$ was increased compared to untreated 5xFAD animals. C) The FA soluble fraction was unaffected by therapeutic treatment. Preventive treatment with mIgG1 resulted in a significant reduction of FA soluble $\mathrm{A} \beta$ compared to untreated $5 \times \mathrm{XAD}$ animals.

Total A $\beta$ in TBS soluble, triton soluble and formic acid soluble fractions was also measured using ELISA. In the soluble fraction, no difference between any of the groups could be detected $(\mathrm{p}=0.052)$ (see Figure 3.6A). Unexpectedly, $A \beta$ was increased in the triton fraction of both prevention groups compared to untreated $5 \mathrm{xFAD}$ animals $(\mathrm{p}<0.01)$, while triton soluble $\mathrm{A} \beta$ was decreased in both therapeutic groups compared to untreated $5 \mathrm{xFAD}$ animals $(\mathrm{p}<0.01)$ (see Figure 3.6B). Interestingly, preventive treatment with mIgG1 caused a reduction in formic acid soluble $A \beta(p=0.035)$, suggesting that antibody treatment might shift A $\beta$ between fractions. MIgG2a also caused a (nonsignificant) reduction in the formic acid fraction of $A \beta$, but this difference was lower, 
which was consistent with the results from immunohistochemistry (see Figure 3.5).

\subsubsection{REDUCTION IN ASTROGLIOSIS IS ISOTYPE DEPENDENT}

$\mathrm{AD}$ is also characterized by increased gliosis. To investigate the effects on gliosis microglial and astrocyte activation were assessed using immunofluorescence with the markers Ibal (microglia) and GFAP (astrocytes). Additionally, the amount of plaque associated microglia and astrocytes was assessed.

No significant differences could be found in microglial density between any of the groups, although microglial activation was increased in the untreated animals and animals therapeutically treated with mIgG1 compared to other groups (see Figure 3.7A). There were also no differences present in plaque associated microglia (see Figure 3.7D). Analysis of specific subregions of the brain also showed no significant differences in microgliosis between groups (data not shown).

Untreated 5xFAD animals showed strong astrogliosis compared to WT animals $(\mathrm{p}<0.01)$. Animals treated with mIgG2a had a significant reduction in astrogliosis in whole brain compared to untreated animals. In the hippocampus, treatment with mIgG2a resulted in less astrocyte activation compared to treatment with mIgG1 $(\mathrm{p}=0.02)$ (see Figure 3.7D). In the whole brain, no significant differences were present in plaque associated astrocytes between any of the groups $(p=0.132)$. However, in the hippocampus, both therapeutic and preventive treatment with mIgG2a significantly reduced plaque-associated astrocytes compared to untreated 5xFAD animals $(\mathrm{p}=0.02)$ (see Figure 3.7). MIgG1 had no effect on astrogliosis. 


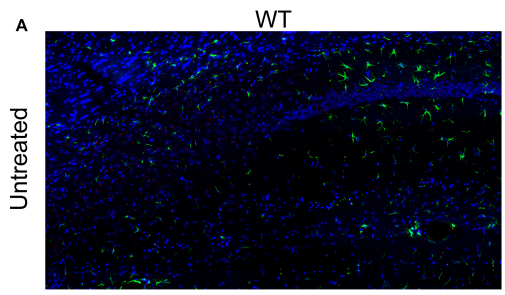

$\lg \mathrm{G} 1$

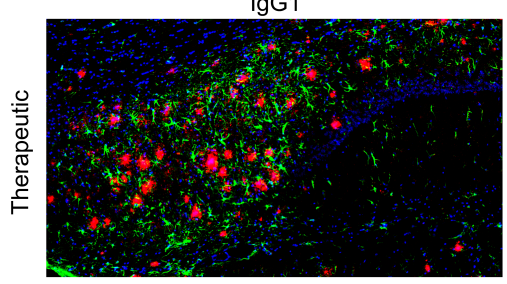

$\lg G 1$

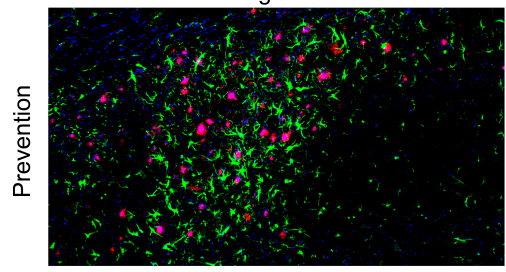

Amyloid $\beta$ Astrocytes Nuclei
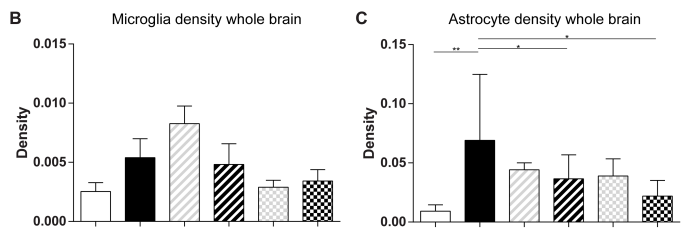

E

E Plaque associated microglia whole brain $F$

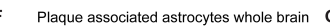

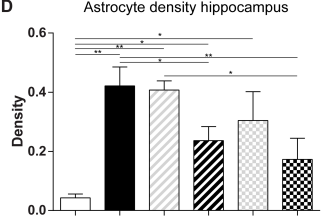

5xFAD

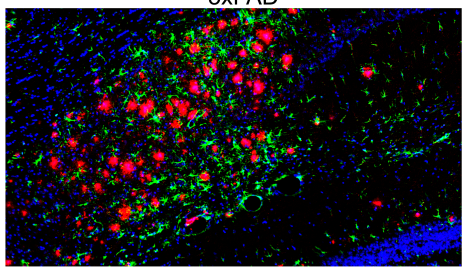

$\lg 2 a$

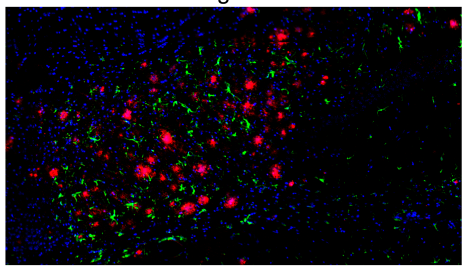

$\lg \mathrm{g} 2 \mathrm{a}$

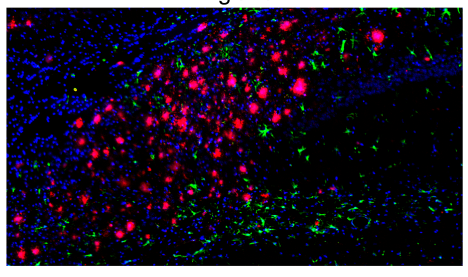

$500 \mu \mathrm{m}$

Plaque associated astrocytes hippocampus

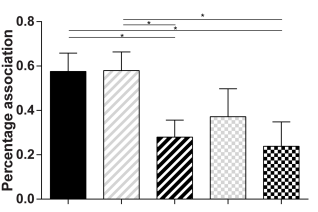

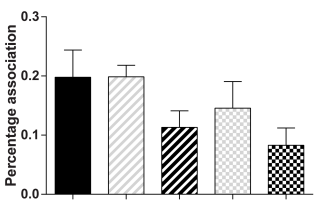

$\begin{array}{ll}\square \text { WT } & \square \text { mlgG1 Therapeutic } \square \text { mlgG1 Preventive } \\ \text { 5xFAD } & \mathbf{Z} \text { mlgG2a Therapeutic } \$ \text { mlgG2a Preventive }\end{array}$

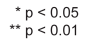

Figure 3.7: Gliosis was assessed by measuring reactivity against Ibal (microglia) and GFAP (astrocytes) in different brain regions using immunofluoresence. Plaque associated astrocytes and microglia were assessed by double labeling of $A \beta$ and Ibal or GFAP. A) Representative images of subiculum show a significant reduction in astrocytes (green) present in animals treated with mIgG2a. In addition, less astrocytes are present near plaques (red) in mIgG2a treated animals. B) No significant differences were present in microglia density in the brain ( $\mathrm{n}_{\mathrm{WT}}=3, \mathrm{n}_{5 \mathrm{xFAD}}=8, \mathrm{n}_{\text {mIgG1Ther }}=7, \mathrm{n}_{\text {mIgG2aTher }}=9, \mathrm{n}_{\text {mIgG1Prev }}=4, \mathrm{n}_{\text {mIgG2aPrev }}=6$ ). C-D) Astrocyte density was significantly lowered by treatment with mIgG2a 3D6, but not with mIgG1 3D6 $\left(\mathrm{n}_{\mathrm{WT}}=4, \mathrm{n}_{5 \mathrm{xFAD}}=6\right.$, $\left.\mathrm{n}_{\text {mIgG1Ther }}=4, \mathrm{n}_{\text {mIgG2aTher }}=8, \mathrm{n}_{\text {mIgG1Prev }}=4, \mathrm{n}_{\text {mIgG2aPrev }}=6\right)$ E) There were no differences in the amount of plaque associated microglia $\left(n_{5 x F A D}=8, n_{\text {mIgG1Ther }}=7, n_{\text {mIgG2aTher }}=9, n_{\text {mIgG1Prev }}=4, n_{\text {mIgG2aPrev }}=6\right)$. F-G) In the whole brain there was no difference in plaque associated astrocytes, but in the hippocampus mIgG2a treatment caused a significant reduction in plaque associated astrocytes. $\left(\mathrm{n}_{\mathrm{WT}}=4, \mathrm{n}_{5 \mathrm{xFAD}}=6, \mathrm{n}_{\mathrm{mIgG1Ther}}=4\right.$, $\mathrm{n}_{\text {mIgG2aTher }}=8, \mathrm{n}_{\text {mIgG1Prev }}=4, \mathrm{n}_{\text {mIgG2aPrev }}=6$ ) Error bars denote SEM. 


\subsection{DISCUSSION}

In this study, the isotype dependent effects of mAb 3D6 were investigated in the 5xFAD mouse model. Additionally, a preventive and therapeutic regimen were compared. Our results show that preventive treatment reduced anxiety as assessed by the EZM. Additionally, preventive immunotherapy also had stronger plaque reducing effects, as assessed by total density and by plaque size. Interestingly, triton soluble $A \beta$ was increased in the preventive immunotherapy groups, while formic acid soluble $A \beta$ was decreased by preventive treatment with mIgG1. Therapeutic treatment on the other hand reduced triton soluble $A \beta$, while it had no effect the formic acid fraction. We also found that treatment with mIgG2a 3D6 reduced astrogliosis.

Surprisingly we find that anti-inflammatory immunotherapy with the mIgG1 isotype results in a worsened memory as assessed by contextual fear conditioning. Finally, as also reported in a recent publication, immunotherapy can be lethal, raising concerns for passive immunotherapy in human trials [17].

Previous studies using $\mathrm{F}\left(\mathrm{ab}^{\prime}\right)_{2}$ 3D6 showed that non-Fc mediated mechanisms are involved in clearing amyloid plaques in a different mouse model of $\mathrm{AD}$, and we confirm that mIgG1 is equally effective in clearing plaques than mIgG2a [18]. Another study that compared different anti-amyloid antibodies showed that mIgG1 antibodies were less effective in clearing plaques, but their results might have been confounded by the fact that different antibodies were used, and the epitope and affinity might play a crucial role in addition to the isotype [19].

For the moment, human immunotherapy trials have been unsuccessful in halting AD. Our results support the notion that preventive immunotherapy trials are potentially more effective than therapeutic trials. Only when treatment was started early in the disease process, were we able to reduce latency to enter the closed arm back to baseline levels in the EZM. These results offer hope for current ongoing preventive trials in humans.

Surprisingly, we found that treatment with mIgG1 3D6 increased memory problems in the 5xFAD animal model. A possible explanation could be found in the dustraising effect previously reported for $\mathrm{N}$-terminal antibodies [20]. The dust-raising effect states that $A \beta$ oligomers are more toxic that fibrils, and transforming $A \beta$ from its less neurotoxic fibrillary form in plaque deposits into its more neurotoxic oligomeric form might actually worsen the disease. Treatment with mIgG2a 3D6 would possible cause oligomeric $A \beta$ to be removed by Fc-mediated phagocytosis, while this would not be the case when treating with mIgG1 3D6, causing the freed oligomeric $A \beta$ to exert its more neurotoxic effects. Additionally, anti-inflammatory treatment using ibuprofen has also been reported to worsen behavioral outcomes in this mouse model [21].

Interestingly, our results show that mIgG2a 3D6, but not mIgG1 3D6, dampens astrogliosis, while the effect on microgliosis is less pronounced. While it has been shown that astrocytes express $\mathrm{Fc} \gamma$-receptor [22, 23], their role in neurodegenerative disorders and especially in immunotherapy has not been studied in detail. However, given the active role that astrocytes play in the maintenance of the blood brain barrier (BBB), it is not surprising that they might play an active role in immunotherapy.

Similar to a recent preclinical immunotherapy study we also report increased mortality in animals receiving therapy with 3D6 [17]. While in the study of Mably et 
al. the cause of the mortality was not determined, we have found that most animals developed neutralizing antibodies against our administered antibody. Given the rapid onset of death and the observed symptoms, it is likely that animals subsequently died from passive systemic anaphylaxis (PSA), although we could not confirm this by for instance temperature measurements. We further showed that the PSA is IgG-, and not IgE-mediated, and one study indicated that the severity of the reaction is related to the amount of antigen present [24]. Possibly, this reaction was triggered by the use of our chimeric antibody.

A limitation of our study is that the mice only showed a weak phenotype in our behavioral paradigm. Only in 1 out of 4 tests, a difference was observed between WT and untreated 5xFAD animals. One possible reason for this could be the employed breeding scheme we utilized. Instead of using WT B6/SJL F1 hybrids [15], WT B6/SJL F3 mice were used to generate offspring. Additionally, the Pde $6 \mathrm{~b}^{\text {rdl }}$ was bred out of our colony. Both factors might have resulted in a weaker phenotype, as is also reported by Jackson Laboratories for the 5xFAD strain on congenic C57/B6 background.

Another limitation is that our results might have been confounded by that fact that immunotherapy caused PSA. This acute state of systemic inflammation might have influenced the inflammatory state in the CNS, which might explain why there were no differences in microglial activation. Additionally, neutralizing antibodies against the administered 3D6 antibodies could have lowered the effective dose reaching the brain, resulting in less efficacy.

In conclusion, our study confirms that preventive immunotherapy treatment might be more effective than therapeutic treatment. In addition, isotype dependent effects of immunotherapy should be further investigated, since our results suggest that the isotype affects the behavioral phenotype, independent of plaque load. This is especially important keeping in mind the disappointing results of the hIgG4 (mIgG1 equivalent) antibody crenezumab in its phase II trial [25]. Additionally, it has been shown that monoclonal antibody treatment can cause PSA in up to $70 \%$ of the patients [24], and our results show that this could potentially occur in passive amyloid immunotherapies. 


\section{REFERENCES}

[1] Schneider LS, Mangialasche F, Andreasen N, Feldman H, Giacobini E, Jones R, et al. Clinical trials and latestage drug development for Alzheimer's disease: an appraisal from 1984 to 2014 [Journal Article]. Journal of Internal Medicine. 2014;275(3):251283. Available from: http://dx.doi.org/10.1111/joim.12191.

[2] Wisniewski T, Goni F. Immunotherapeutic Approaches for Alzheimer's Disease [Journal Article]. Neuron. 2015;85(6):1162-1176. Available from: http://www . sciencedirect.com/science/article/pii/S0896627314011738.

[3] Morgan D. Immunotherapy for Alzheimer's disease [Journal Article]. Journal of internal medicine. 2011;269(1):54-63.

[4] Salloway S, Sperling R, Fox NC, Blennow K, Klunk W, Raskind M, et al. Two Phase 3 Trials of Bapineuzumab in MildtoModerate Alzheimer's Disease [Journal Article]. New England Journal of Medicine. 2014;370(4):322-333. Available from: http:// www.nejm.org/doi/full/10.1056/NEJMoa1304839.

[5] Delnomdedieu M, Duvvuri S, Li DJ, Atassi N, Lu M, Brashear HR, et al. FirstInHuman safety and longterm exposure data for AAB003 (PF05236812) and biomarkers after intravenous infusions of escalating doses in patients with mild to moderate Alzheimer's disease [Journal Article]. Alzheimer's Research \& Therapy. 2016;8(1):1-10. Available from: http://dx.doi.org/10.1186/ s13195-016-0177-y.

[6] Carrillo MC, Brashear HR, Logovinsky V, Ryan JM, Feldman HH, Siemers ER, et al. Can we prevent Alzheimer's disease? Secondary "prevention" trials in Alzheimer's disease [Journal Article]. Alzheimer's \& Dementia. 2013;9(2):123131.e1. Available from: http://www.sciencedirect.com/science/article/ pii/S1552526012025873.

[7] Fleisher AS, Chen K, Quiroz YT, Jakimovich LJ, Gomez MG, Langois CM, et al. Florbetapir PET analysis of amyloid $\beta$ deposition in the presenilin 1 E280A autosomal dominant Alzheimer's disease kindred: a crosssectional study [Journal Article]. The Lancet Neurology. 2012;11(12):1057-1065. Available from: http: //www.sciencedirect.com/science/article/pii/S1474442212702272.

[8] Reiman EM, Langbaum J, Fleisher AS, Caselli RJ, Chen K, Ayutyanont N, et al. Alzheimer's Prevention Initiative: a plan to accelerate the evaluation of presymptomatic treatments [Journal Article]. Journal of Alzheimer's Disease. 2011;26(s3):321-329.

[9] Duncan AR, Winter G. The binding site for Clq on IgG [Journal Article]. Nature. $1988 ; 332$.

[10] Shields RL, Namenuk AK, Hong K, Meng YG, Rae J, Briggs J, et al. High resolution mapping of the binding site on human IgG1 for Fc?RI, Fc?RII, Fc?RIII, and FcRn and design of IgG1 variants with improved binding to the Fc?R [Journal Article]. Journal of Biological Chemistry. 2001;276(9):6591-6604. 
[11] Sambrook J, Fritsch EF, Maniatis T. Molecular cloning. vol. 2. Cold spring harbor laboratory press New York; 1989.

[12] Detrait E, Brohez C, Hanon E, Ryck M. Automation of continuous spontaneous alternation to increase the throughput for in vivo screening of cognitive enhancers. Optimization of the Ethovision system for the Ymaze test in mice. In: Proceedings of Measuring Behavior; 2010. p. 141-144.

[13] Edelstein AD, Tsuchida MA, Amodaj N, Pinkard H, Vale RD, Stuurman N. Advanced methods of microscope control using microManager software [Journal Article]. Journal of Biological Methods. 2014;1(2):e10.

[14] Schindelin J, ArgandaCarreras I, Frise E, Kaynig V, Longair M, Pietzsch T, et al. Fiji: an opensource platform for biologicalimage analysis [Journal Article]. Nature methods. 2012;9(7):676-682.

[15] Oakley H, Cole SL, Logan S, Maus E, Shao P, Craft J, et al. Intraneuronal $\beta$ Amyloid Aggregates, Neurodegeneration, and Neuron Loss in Transgenic Mice with Five Familial Alzheimer's Disease Mutations: Potential Factors in Amyloid Plaque Formation [Journal Article]. The Journal of Neuroscience. 2006;26(40):1012910140. Available from: http://www.jneurosci.org/content/26/40/10129. abstract.

[16] Buccafusco JJ. Methods of behavior analysis in neuroscience. CRC Press; 2000.

[17] Mably AJ, Liu W, Mc Donald JM, Dodart JC, Bard F, Lemere CA, et al. AntiA $\beta$ antibodies incapable of reducing cerebral $A \beta$ oligomers fail to attenuate spatial reference memory deficits in J20 mice [Journal Article]. Neurobiology of Disease. 2015;82:372-384. Available from: http://www.sciencedirect.com/science/ article/pii/S0969996115300127.

[18] Bacskai BJ, Kajdasz ST, McLellan ME, Games D, Seubert P, Schenk D, et al. Non-Fc-mediated mechanisms are involved in clearance of amyloid $\beta$ in vivo by immunotherapy [Journal Article]. The Journal of neuroscience. 2002;22(18):78737878.

[19] Bard F, Barbour R, Cannon C, Carretto R, Fox M, Games D, et al. Epitope and isotype specificities of antibodies to ßamyloid peptide for protection against Alzheimer's diseaselike neuropathology [Journal Article]. Proceedings of the National Academy of Sciences. 2003;100(4):2023-2028.

[20] Liu YH, Bu XL, Liang CR, Wang YR, Zhang T, Jiao SS, et al. An Nterminal antibody promotes the transformation of amyloid fibrils into oligomers and enhances the neurotoxicity of amyloidbeta: the dustraising effect [Journal Article]. Journal of neuroinflammation. 2015;12(1):153.

[21] Hillmann A, Hahn S, Schilling S, Hoffmann T, Demuth HU, Bulic B, et al. No improvement after chronic ibuprofen treatment in the 5XFAD mouse model of 
Alzheimer's disease [Journal Article]. Neurobiology of Aging. 2012;33(4):833.e39833.e50. Available from: http://www.sciencedirect.com/science/article/ pii/S0197458011003216.

[22] Li YN, Qin XJ, Kuang F, Wu R, Duan XL, Ju G, et al. Alterations of Fc gamma receptor I and Toll-like receptor 4 mediate the antiinflammatory actions of microglia and astrocytes after adrenaline induced blood brain barrier opening in rats [Journal Article]. Journal of neuroscience research. 2008;86(16):3556-3565.

[23] Nitta T, Yagita H, Sato K, Okumura K. Expression of Fc $\gamma$ receptors on astroglial cell lines and their role in the central nervous system [Journal Article]. Neurosurgery. 1992;31(1):83-88.

[24] Jiao D, Liu Y, Lu X, Pan Q, Zheng J, Liu B, et al. Characteristics of anaphylaxis inducing IgG immune complexes triggering murine passive systemic anaphylaxis [Journal Article]. Allergy. 2013;68(2):236-245.

[25] Cummings J, Cho W, Ward M, Friesenhahn M, Brunstein F, Honigberg L, et al. A randomized, doubleblind, placebocontrolled phase 2 study to evaluate the efficacy and safety of crenezumab in patients with mild to moderate Alzheimer's disease [Journal Article]. Alzheimer's \& Dementia: The Journal of the Alzheimer's Association. 2014;10(4):P275. 



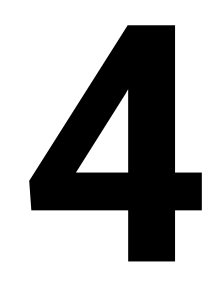

\section{IN VIVO PET IMAGING IN AN AD MODEL}

Jo A.A. Stevens, Pilar Martinez-Martinez, Ludwig Dubois, Danielle J. Vugts, Thijs E. Nell, Simone M. Crivelli, Marc H. De Baets, Wim A. Buurman, Peter C. Molenaar, Boudewijn Brans, Pieter Jelle Visser, Guus A. van Dongen, Mario Losen Submitted 


\section{ABSTRACT}

A hallmark of Alzheimer's disease (AD) is the accumulation of amyloid beta $(A \beta)$ peptide in the brain. Several $A \beta$ antibodies are currently tested in clinical trials as a possible treatment for $\mathrm{AD}$. We investigated the possibility to use a radiolabeled $\mathrm{A} \beta$ antibody (bapineuzumab) for in vivo imaging of $\mathrm{A} \beta$ pathology in the brain of an $\mathrm{AD}$ mouse model (5xFAD).

Recombinant bapineuzumab and an isotype control antibody were labeled with 89-zirconium and administered to 3-, 6- and 9-month old female 5xFAD or wild type (WT) control mice. Already at 3 months of age, when plaque pathology was still limited, specific uptake of bapineuzumab in 5xFAD animals could be detected in vivo using PET scans. In addition, gamma counting showed an age-related accumulation of bapineuzumab in the brain of 5xFAD animals, which correlated with plaque load. No difference between 5xFAD and WT animals was found when using an isotype control immunoglobulin, or when blocking labelled bapineuzumab in vivo with an excess of unlabeled bapineuzumab. Results were validated by both autoradiography and immunofluorescence, with the latter showing co-localization of bapineuzumab in $A \beta$ plaques.

This study demonstrates that uptake of 89-zirconium labelled bapineuzumab in the brain can be specifically imaged in vivo using PET. 


\subsection{INTRODUCTION}

Alzheimer's disease $(\mathrm{AD})$ is the most common cause of dementia and characterized by a buildup of extracellular plaques, containing aggregates of the protein amyloid-beta (A $\beta$ ), and intracellular tangles, containing tau protein. In the last years, the amyloid cascade hypothesis, which states that the buildup of $A \beta$ is the cause of $A D$, has been refined to postulate that other forms of $A \beta$, such as pyroglutamate or oligomeric $A \beta$, are the more neurotoxic species. In vivo positron emission tomography (PET) tracers, such as ${ }^{18} \mathrm{~F}$ florbetapir, ${ }^{18} \mathrm{~F}$-florbetaben and ${ }^{18} \mathrm{~F}$-flutemetamol (Vizamyl), can only detect fibrillary $A \beta$ deposits, while these other $A \beta$ assemblies can only be detected post-mortem using specific antibodies.

Immuno-PET combines the high specificity of antibodies with the sensitivity of PET. Furthermore, by using a long-lived isotope such as zirconium-89, the in vivo distribution of an antibody can be monitored over a period of several days [1,2]. While immunoPET has been widely used in the field of oncology, applications in the central nervous system (CNS) are still not common. This is probably due to a common misconception that antibodies do not cross the blood brain barrier (BBB) [3]. IgG concentrations in cerebrospinal fluid (CSF) are about 500 times lower than in serum, but they still cross the $\mathrm{BBB}$ with a rate of $0.018 \mathrm{mg} / \mathrm{min}$, have a turnover of $0.0036 \mathrm{mg} / \mathrm{min}$ (which is comparable to the CSF turnover, $\sim 4$ times per day), and IgG comprises about $9.8 \%$ of total CSF protein $[4,5]$. Thus while the brain penetration of antibodies is not high compared to traditional PET tracers, highly specific and high affinity antigen-antibody interactions can potentially be utilized to valuably complement the diagnostic potential of other tracers.

In this study, we evaluated the potential application of immuno-PET in an animal model for $\mathrm{AD}$. We used the anti-A $\beta$ antibody bapineuzumab, which binds to the $\mathrm{N}$ terminal part of $A \beta$ and recognizes monomers, oligomers and fibril, although it has a higher affinity for fibrils. Bapineuzumab has been tested in several clinical trials $[6,7,8]$ : while therapeutically not effective to prevent progression of $\mathrm{AD}$, it has been found safe for humans at moderate doses [8]. Two possible reasons for its ineffectiveness might be the fact that up to $20 \%$ of included patients might not have had $\mathrm{AD}$ or that the treatment started too late $[9,10]$.

We used the 5xFAD mouse model for $\mathrm{AD}$, which has five familial $\mathrm{AD}$ mutations (APPSwedish, APPFlorida, APPLondon and PSEN1*M146L*L286V) and is characterized by early-onset plaque pathology starting at 2-3 months of age [11]. ${ }^{89} \mathrm{Zr}$-bapineuzumab was administered at various disease stages followed by in vivo microPET imaging, biodistribution, autoradiography and immunohistochemistry analysis. We observed that as early as 3 months of age, when $A \beta$ pathology was still modest, there was significantly more ${ }^{89} \mathrm{Zr}$-bapineuzumab uptake in the brain of $\mathrm{AD}$ mice. Our results show great promise of ${ }^{89} \mathrm{Zr}$-immuno-PET for imaging of specific antibody binding to $\mathrm{A} \beta$ pathology in the brain. 


\subsection{Material AND Methods}

\subsubsection{ANIMALS}

B6/SJL wild type (WT) and 5xFAD animals were obtained from Jackson laboratory (stock 6554) and bred hemizygously [11] using F3 WT mice. Female mice were used at the ages of 3, 6 and 9 months (numbers are listed in Table 4.1). Mice were housed socially on a 12 hour day-night cycle and had ad libitum access to food and water. All experiments were approved by the Animal Welfare Committee of Maastricht University and followed the laws, rules and guidelines of the Netherlands.

Table 4.1: Number of animals per experimental group

\begin{tabular}{cccc}
\hline Age (months) & ${ }^{89}$ Zr-mAb 637 & ${ }^{89}$ Zr-bapineuzumab & $\begin{array}{l}{ }^{89} \text { Zr-bapineuzumab/ } \\
\text { bapineuzumab }(1 / 10)\end{array}$ \\
\hline & WT & 5 XFAD & WT \\
3 & 5 & 5 & 5 \\
6 & 5 & 5 & 5 \\
9 & 5 & 4 & 4 \\
\hline
\end{tabular}

\subsubsection{ANTIBODY LABELING}

Antibodies were produced recombinantly by transient transfection of HEK cells. Human IgG (IgG1) was chosen to facilitate ex vivo detection of injected antibody. The light and heavy chain coding sequences of bapineuzumab [12] were synthesized de novo (Invitrogen-Geneart) and cloned in pFuse IgG expression vectors (Invivogen). Plasmids were produced at large scale in E. coli grown in a Bioflo 110 fermentor (New Brunswick) and purified using a CIM DEAE column (BIA Separations) according to manufacturer instructions. For production of antibody, HEK293-F cells (Invitrogen) were grown to confluence in a 4-layer Nunc cell factory (Thermo Scientific) in Freestyle 293 medium (Invitrogen). Cells were subsequently transfected using $25 \mathrm{kDa}$ linear PEI (Polysciences) dissolved in purified water. DNA-PEI complexes were prepared using a 1:4 DNA:PEI ratio. Four hours after transfection, valproic acid (Sigma-Aldrich) was added to a concentration of $4 \mathrm{mM}$ and supernatant was harvested after 9-10 days of incubation. Antibody was subsequently purified on an AKTA explorer (GE Healthcare Life Sciences) with a MabSelect Protein A column (GE Healthcare Life Sciences) immediately followed by a buffer exchange using a Sephadex column (GE Healthcare Life Sciences). Antibody purity was confirmed by Coomassie blue staining and concentration was measured using a Lowry protein assay (Biorad). The recombinant antibody mAb 637 (monoclonal human IgG1, directed against human muscle AChR $\alpha$ l subunit) was used as an isotype control; cloning and production are described elsewhere [13]. MAb 637 was tested for immuno-reactivity in mouse muscle and brain, and was found to be non-reactive.

Affinity of labeled bapineuzumab was determined by ELISA. Briefly, $250 \mathrm{pg} /$ well recombinant $\mathrm{A} \beta_{1-42}$ (rPeptide) was coated overnight at $4{ }^{\circ} \mathrm{C}$ on a HiBind 96-well plate (Greiner Bio-One). The plate was washed, blocked with $4 \%$ non-fat dry milk (Biorad) and subsequently incubated with labeled or unlabeled bapineuzumab. After washing, 
the plate was incubated using anti-human IgG HRP (Jackson Immunoresearch) and developed using tetramethylbenzidine (TMB). The OD was measured on a Victor plate reader (Perkin Elmer) at $450 \mathrm{~nm}$, and EC50 (apparent affinity) value was calculated using Graphpad Prism 5. This showed that both labeled and unlabeled bapineuzumab bound immobilized amyloid beta with a similar apparent affinity (see Fig. S1C). Antibody specificity before and after labelling was also confirmed using immunohistochemistry on APP/PSEN1 mouse brain slices: labeled bapineuzumab retained binding on APP/PSEN1 sections (not shown).

Antibodies were labeled with ${ }^{89} \mathrm{Zr}$ using described methods [14] with an average overall labeling yield of $78.6 \%$ (81.2\% for bapineuzumab and $76 \%$ for mAb 637 ), a radiochemical purity always higher than $99 \%$ and a specific activity typically in the range of $\sim 37 \mathrm{MBq} / \mathrm{mg}$.

A total of $100 \mu \mathrm{g}$ of antibody $(0.5 \mu \mathrm{g} / \mu \mathrm{L}$, dissolved in saline) was injected intraperitoneally (i.p.) while animals were under isoflurane anesthesia. Average injected activity was $3.56 \pm 0.24 \mathrm{MBq}$. For blocking experiments, animals were injected with $1 \mathrm{mg}$ of unlabeled bapineuzumab, together with $100 \mu \mathrm{g}$ of ${ }^{89} \mathrm{Zr}$-bapineuzumab.

\subsubsection{IMAGING AND DATA ANALYSIS}

PET images were acquired using a Siemens MicroPET Focus 120 (Siemens Healthcare) with an axial field of view (FOV) of $7.6 \mathrm{~cm}$ and a resolution of about $1.3 \mathrm{~mm}$. Animals were anesthetized using isoflurane $\left(2.5 \%\right.$ in $\left.\mathrm{O}_{2}\right)$, and body temperature was maintained using an electrical heating pad controlled by an anal temperature probe. The animal was positioned supine and data was acquired for 30 minutes. Acquired data were corrected for dead time, random coincidences and decay. Images were reconstructed using OSEM2D. Volumes of interest were analyzed with Carimas 2.9 [15, 16].

The head was first coregistered using normalized cross correlation to a CT template scan that was acquired as described [17]. Manual realignment was performed if the automatic coregistration algorithm failed. Next, a volume of interest (VOI) was selected in the CT template and mean $\mathrm{Bq} / \mathrm{mL}$ was calculated of the VOI. The VOI had a volume of $25 \mu \mathrm{L}$ and was placed centrally in the brain in order to minimize partial volume effects caused by high activity in the surrounding tissues (see Fig. 1); especially bone (and thus skull) have been shown to have high uptake of ${ }^{89} \mathrm{Zr}$ [18]. The standardized uptake value (SUV) was calculated by correcting the mean activity of the VOI (in Bq/ml) for body weight (in g), injected dose (Bq) and decay.

\subsubsection{BIODISTRIBUTION}

Animals were sacrificed by cervical dislocation under isoflurane anesthesia after the last scan. The brain was removed and one hemisphere was rapidly frozen by liquid nitrogen. The other hemisphere was macrodissected into hindbrain (cerebellum and brain stem), bulbus, hippocampus and cortex. 50-100 $\mu \mathrm{L}$ of blood was obtained from the heart. For analysis of antibody uptake in other tissues, the following organs were dissected: salivary glands, cervical lymph nodes, skin, eyes, spleen, intestines, liver, stomach, bladder, kidneys, lungs and heart. The body was further divided into limbs, head and trunk. Radioactivity content in all these body parts was measured using a 2470 Wizard $^{2}$ gamma counter (Perkin Elmer) for 2 minutes per sample. Results were corrected 
for radioactive decay and expressed as percentage of total injected dose (in Bq) per gram of tissue $(\% \mathrm{ID} / \mathrm{g})$.

\subsubsection{AUTORADIOGRAPHY, IMMUNOFLUORESCENCE AND MICROSCOPY} The contralateral frozen hemisphere was cut on a cryostat at $-17^{\circ} \mathrm{C}$. For autoradiography, $50 \mu \mathrm{m}$ sections were incubated on a phosphor film for about 2 weeks. The film was read out by a Typhoon FLA 7000 (Perkin Elmer).

For immunohistochemistry, $16 \mu \mathrm{m}$ thick sections were stained with biotinylated goat anti-human IgG (Brunschwig, 1:500). Plaque load was determined by incubation with biotinylated bapineuzumab (1:2000). For co-localization studies, a double staining was performed with mouse-anti human IgG Alexa 594 (Jackson Immunoresearch, 209-585082, 1:250) and biotinylated human anti-A $\beta$ mAb 20C2 (recombinantly produced [19], $1: 1000)$.

Photomicrographs were acquired using $\mu$ Manager software [20] on a BX51WI spinning disk confocal fluorescence microscope (Olympus) with a EM-CCD C9100 digital camera (Hamamatsu) and a QIcam color camera (QImaging). Images were background corrected and stitched using a plug-in [21] of ImageJ software (www.imagej.nih.gov/ij/).

For quantification of plaques two midsagittal sections per animal were used, spaced $160 \mu \mathrm{m}$ apart. Images were analyzed using ImageJ 1.49. First, regions-of-interest (ROIs) were manually delineated. These ROIs comprised the cortex, subiculum, hippocampus, cerebellum, bulbus, brainstem and the midbrain combined with the striatum. Next, a median filter was applied, and by using the threshold function, images were converted to binary images. Using the particle analyzer tool, plaque area was determined and divided by the total ROI area. For colocalization analysis, average fluorescence intensity of bapineuzumab and 20C2 was measured in the plaque areas (defined by 20C2 immunoreactivity). The same settings were used for all images.

\subsubsection{STATISTICAL ANALYSIS}

Statistical analysis was performed using SPSS Statistics 22 software (IBM). PET and biodistribution data were analyzed using a 3-way ANOVA, with animal age, genotype, and antibody as independent factors. If interaction was present, data were analyzed by a pair-wise comparison of estimated marginal means using Bonferroni correction. Analysis of the optimal imaging time point after injection was performed with a repeated measures model. A p-value of $<0.05$ was considered significant. 


\subsection{RESULTS}

\subsubsection{SPECIFIC ACCUMULATION OF ${ }^{89}$ ZR-BAPINEUZUMAB IN THE $5 X F A D$ BRAIN IN VIVO}

A
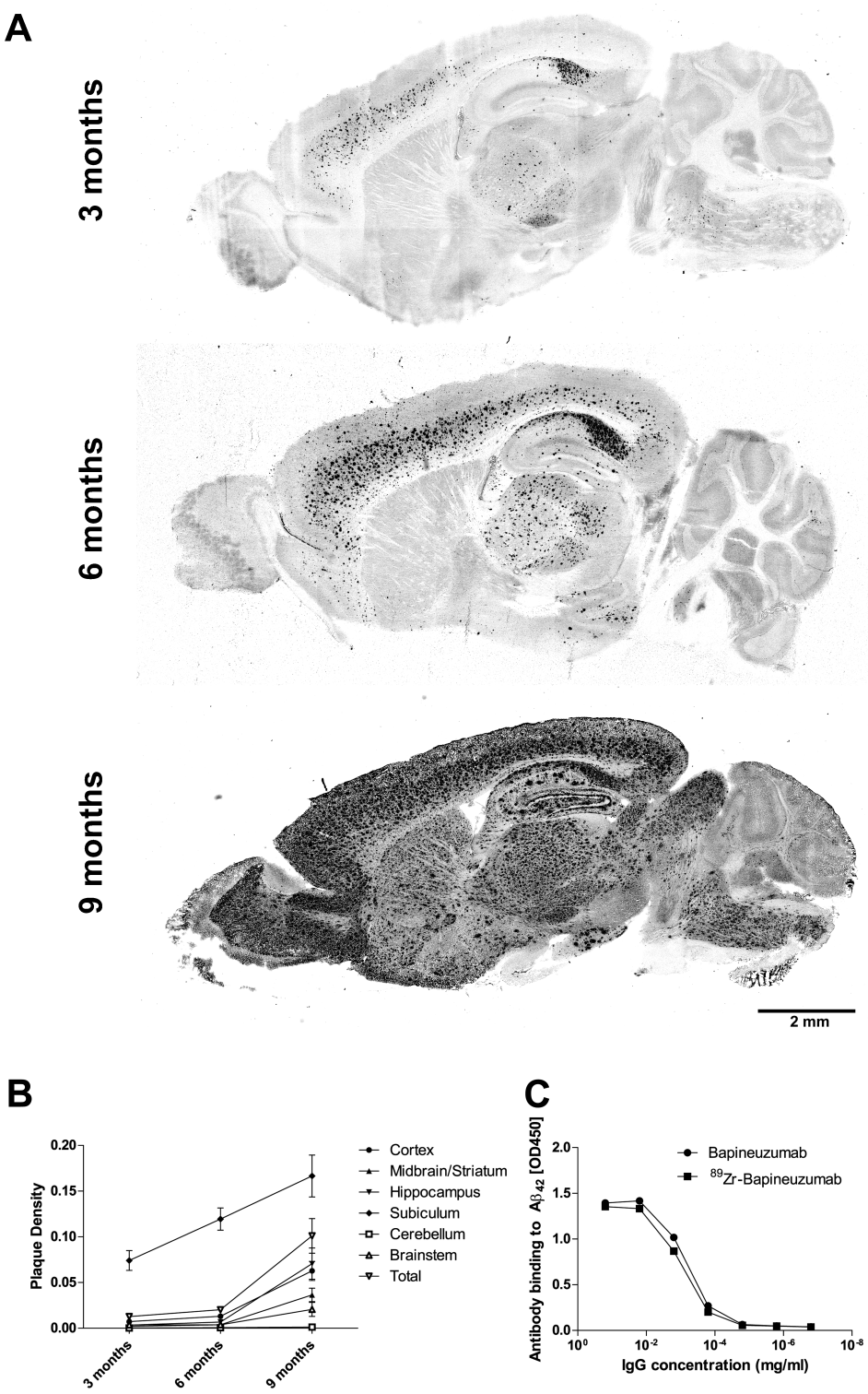

Figure 4.1: Quantification of bapineuzumab labeled plaques in the 5xFAD animal model and affinity determination of ${ }^{89} \mathrm{Zr}$-bapineuzumab. (A) Immunohistochemical staining with bapineuzumab using $5 \times \mathrm{xFD}$ brain sections at different ages. (B) Plaque density was measured in different brain regions using ImageJ. At all ages, the subiculum has the highest plaque density, followed by plaque density in the cortex. (C) A direct ELISA with $A ß_{42}$ shows that the apparent affinity of unlabeled and labeled bapineuzumab are similar. 
As a reference for PET experiments, the $A \beta$ plaque load in brain sections of 3,6 , and 9 months old 5xFAD mice was analyzed by immunohistochemistry with bapineuzumab (Figure 4.1). A $\beta$ pathology started in the subiculum and the cortex and increased with age, in agreement with published data [11]. For PET imaging of $A \beta$ pathology, ${ }^{89} \mathrm{Zr}$ bapineuzumab was administered to 9 month old mice and antibody accumulation was measured over various days. Three days after injection, there was a $\sim 2$-fold difference between the $5 \mathrm{xFAD}$ and WT groups $(\mathrm{p}=0.021)$, and uptake in $5 \mathrm{xFAD}$ animals further increased in the following days (day 5: $\mathrm{p}=0.007$, day $7: \mathrm{p}<0.001$ and day $9: \mathrm{p}<0.001$ ) (see Fig. 4.1).

In 9-month old animals, a blocking experiment was performed to test the specificity of ${ }^{89} \mathrm{Zr}$-bapineuzumab by co-administration of a 10 -fold excess of unlabeled bapineuzumab. In the blocking group, uptake of ${ }^{89} \mathrm{Zr}$-bapineuzumab was reduced to baseline values (Figure 4.2) $(\mathrm{p}=0.908)$ and significantly reduced compared to 5xFAD animals treated with only ${ }^{89} \mathrm{Zr}$-bapineuzumab $(\mathrm{p}<0.001)$.

Isotype control ${ }^{89} \mathrm{Zr}-\mathrm{mAb} 637$ uptake was not statistically different between genotypes ( $\mathrm{p}=0.386$, Figure 4.2 ), while ${ }^{89} \mathrm{Zr}$-bapineuzumab uptake was increased in 5xFAD animals compared to ${ }^{89} \mathrm{Zr}-\mathrm{mAb} 637$ ( $\mathrm{p}<0.001$ ), further proving the specificity of ${ }^{89}$ Zr-bapineuzumab uptake.

A

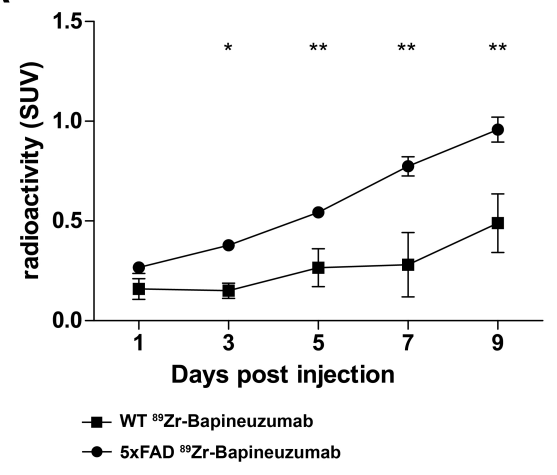

B

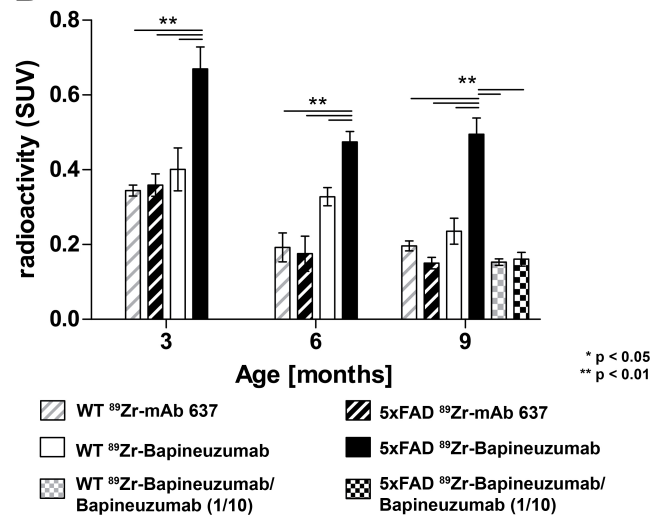

Figure 4.2: Quantification of brain PET scans demonstrated specific uptake of ${ }^{89} \mathrm{Zr}$-bapineuzumab in 5xFAD animals. (A) In 9-month-old 5xFAD and WT animals ( $\mathrm{n}=2$ per group) increased accumulation of ${ }^{89} \mathrm{Zr}$-bapineuzumab in 5xFAD animals was observed 3 days after injection. ${ }^{89} \mathrm{Zr}$-bapineuzumab further accumulated in the brain during the following days. (B) Animals of 6 and 9 months were scanned 5 days after injection, and 3-month-old animals were scanned 8 days after injection. As soon as 3 months of age, increased uptake of ${ }^{89} \mathrm{Zr}$-bapineuzumab was observed in 5xFAD animals injected with ${ }^{89} \mathrm{Zr}$-bapineuzumab and this difference with WT animals remained present at older ages. In animals injected with the isotype control $\left({ }^{89} \mathrm{Zr}\right.$-mAb 637) this difference was not observed. When blocking ${ }^{89} \mathrm{Zr}$-bapineuzumab with a 10 -fold excess of unlabeled bapineuzumab, values were reduced to baseline. Error bars correspond to the SEM. Detailed animal numbers are listed in Table 4.1.

\subsubsection{EARLY DETECTION OF ${ }^{89}$ ZR-BAPINEUZUMAB IN YOUNG $5 X$ FAD MICE} To assess the sensitivity of immuno-PET using ${ }^{89} \mathrm{Zr}$-bapineuzumab, animals were imaged at 3, 6 and 9 months of age using ${ }^{89} \mathrm{Zr}$-bapineuzumab and isotype control ${ }^{89} \mathrm{Zr}$ - 
mAb 637. As early as 3 months of age, when $A \beta$ load was still low, a specific increase in accumulation of ${ }^{89} \mathrm{Zr}$-bapineuzumab was present in the brain; this increase was significant for all ages studied (Figure 4.2, $\mathrm{p}<0.001$ ), although we did not observe an agerelated increase in ${ }^{89} \mathrm{Zr}$-bapineuzumab uptake. No difference was detected between WT and 5xFAD animals treated with the isotype control ${ }^{89} \mathrm{Zr}-\mathrm{mAb} 637(\mathrm{p}=0.582)$ at any age. 



$\square$ WT " ${ }^{\text {BOZr-Bapineuzumab }}$

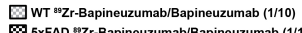
$\begin{array}{rl}* p & p 0.05 \\ * * & p<0.01\end{array}$

Figure 4.3: Overview of biodistribution data from all analyzed organs. The conditions are explained in Fig. 3 and the Biodistribution section in the Material and Methods. In the bulbus a significant difference between WT and 5xFAD animals was found only in 9 month old animals injected with ${ }^{89} \mathrm{Zr}$-bapineuzumab. Furthermore, increased activity in the limbs for both ${ }^{89} \mathrm{Zr}$-bapineuzumab and ${ }^{89} \mathrm{Zr}-\mathrm{mAb} 637$ was found in $5 \mathrm{xFAD}$ animals. 


\subsubsection{BIODISTRIBUTION ANALYSIS CONFIRMED EARLY AGE-SPECIFIC UP- TAKE OF ${ }^{89}$ ZR-BAPINEUZUMAB}
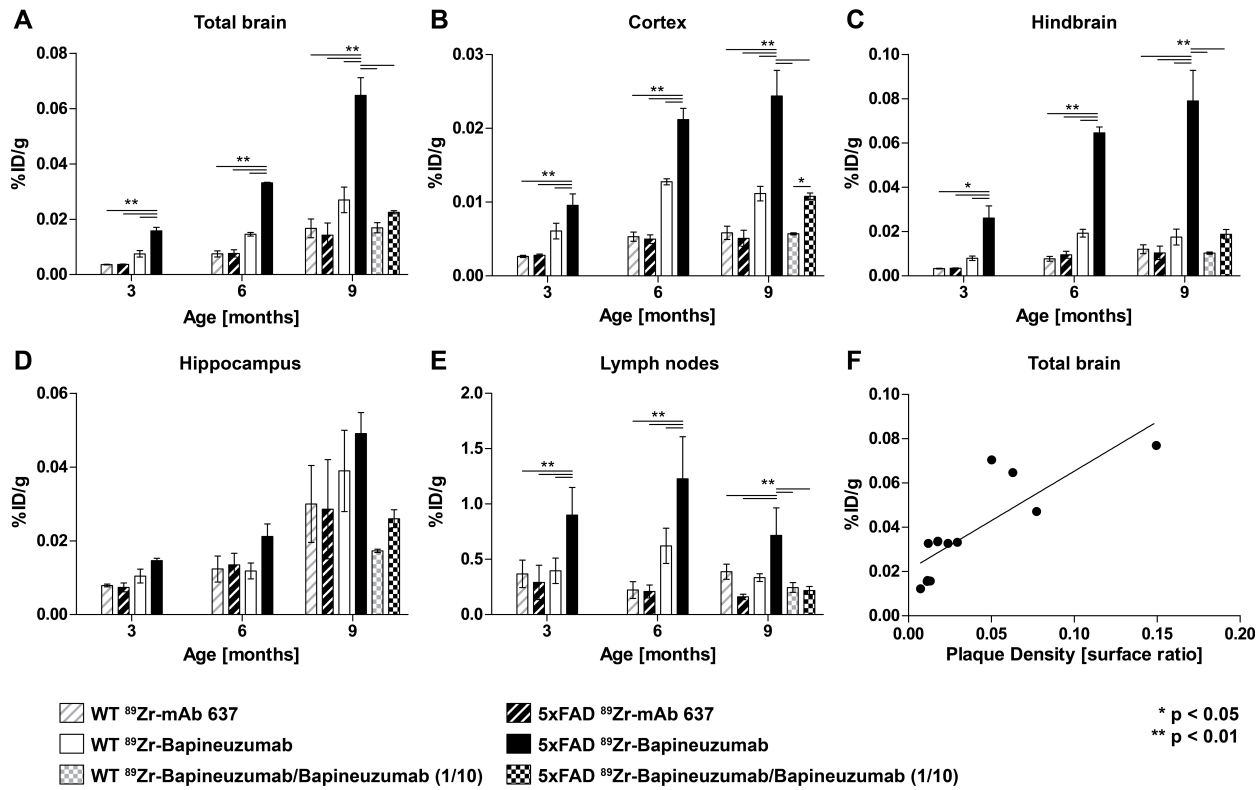

B 5xFAD ${ }^{89} \mathrm{Zr}$-Bapineuzumab/Bapineuzumab (1/10)

Figure 4.4: Biodistribution showed an age dependent increase of ${ }^{89} \mathrm{Zr}$-bapineuzumab uptake in some anatomical regions of the $5 x F A D$ brain. Biodistribution of ${ }^{89} \mathrm{Zr}$-bapineuzumab and isotype control $\left({ }^{89} \mathrm{Zr}-\mathrm{mAb}\right.$ 637) was analyzed in total brain (A), cortex (B), hindbrain (cerebellum and brain stem) (C), hippocampus (D), cervical lymph nodes (E) and the correlation between plaque load and radioactivity (F) (other organs are shown in supplemental Figure S2). In 9-months-old animals, ${ }^{89} \mathrm{Zr}$-bapineuzumab was blocked with a 10 times excess of unlabeled bapineuzumab $\left({ }^{89} \mathrm{Zr}\right.$-bapineuzumab/bapineuzumab (1/10)). (A-C) ${ }^{89} \mathrm{Zr}$-bapineuzumab uptake increased in the brain of aging 5xFAD animals following the reported Aß build up in this model. (D) In the hippocampus no significant differences were found between the experimental groups. (E) In cervical lymph nodes, an increased uptake of ${ }^{89} \mathrm{Zr}$-bapineuzumab was detected in 5xFAD compared to WT mice at all ages. (F) Total brain radioactivity had a strong correlation to the quantified plaque load $(r=0.832)$ All values were normalized for tissue weight, radioactive decay and injected dose (\%ID/g). Error bars correspond to SEM and animal numbers are listed in Table 4.1 .

Five days after antibody administration, uptake was studied in the brain and other organs. In total brain, the uptake (Figure 4.4) closely paralleled the results obtained by immuno-PET (Figure 4.2). In concordance with analysis of the PET scans, ${ }^{89} \mathrm{Zr}$ bapineuzumab brain uptake was significantly higher at all ages for 5xFAD mice compared to age matched WT mice ( $\mathrm{p}=0.02$ at 3 months, and $\mathrm{p}<0.001$ at 6 and 9 months). Additionally, and in contrast to the in vivo PET scans, we found that ${ }^{89} \mathrm{Zr}$-bapineuzumab accumulation in brain tissue increased with age in $5 x F A D$ animals $(\mathrm{p}<0.001)$ (Figure 4.4). A correlation analysis between plaque density and radioactivity in the brain showed a strong correlation $(\mathrm{r}=0.832, \mathrm{p}<0.001$, Fig 4.4). There was no difference at any age in the isotype control groups or when ${ }^{89} \mathrm{Zr}$-bapineuzumab was blocked with cold bapineuzumab, further proving that ${ }^{89} \mathrm{Zr}$-bapineuzumab uptake was specific to $\mathrm{A} \beta$ pathology. 
Because $A \beta$ load in the 5xFAD model differs between brain regions [11] (Figure 4.1), macro dissection of the brain was performed (cortex, hindbrain, hippocampus, and bulbus) and radioactivity in each region was measured. For the cortex, there was a significant difference in ${ }^{89} \mathrm{Zr}$-bapineuzumab uptake between WT and 5xFAD at all analyzed ages $\left(\mathrm{p}<0.001\right.$; Figure $2 \mathrm{~B}$ ). Blocking ${ }^{89} \mathrm{Zr}$-bapineuzumab uptake with an excess of unlabeled bapineuzumab showed a decreased radioactivity compared to $5 x F A D$ animals treated with only ${ }^{89} \mathrm{Zr}$-bapineuzumab $(\mathrm{p}<0.001)$, in line with the results of whole brain. However, when comparing $5 \mathrm{xFAD}$ and WT animals in the blocking group, there was still a small but significant increase of ${ }^{89} \mathrm{Zr}$-bapineuzumab uptake $(\mathrm{p}=0.019)$, indicating incomplete blockage of bapineuzumab binding in the cortex.

Results from the hindbrain (Figure 4.4) showed similar results as the cortex, except that uptake of ${ }^{89} \mathrm{Zr}$-bapineuzumab in the $5 \mathrm{xFAD}$ mice could be completely blocked. At 3 months of age, there was already a 3 -fold increase of ${ }^{89} \mathrm{Zr}$-bapineuzumab uptake in the hindbrain of $5 x F A D$ animals compared to $\mathrm{WT}(\mathrm{p}=0.005)$. A further increased uptake was observed at 6 and 9 months of age of respectively 3.4 and 4.4 fold ( $<<0.001$ for both). For the bulbus, increased uptake of bapineuzumab in 5xFAD animals was observed only at 9 months of age $(p=0.001$, Figure 4.3). No significant differences in uptake were found for the hippocampus (Figure 4.4).

Besides the brain, specific increased uptake of ${ }^{89} \mathrm{Zr}$-bapineuzumab was observed in cervical lymph nodes of $5 x F A D$ mice $(p=0.001$, Figure 4.4 ), suggesting the presence of $A \beta$ in draining lymph nodes of the brain. This was not observed when the isotype control antibody was injected or when ${ }^{89} \mathrm{Zr}$-bapineuzumab uptake was blocked with cold bapineuzumab. Non-specific uptake of ${ }^{89} \mathrm{Zr}$-bapineuzumab and ${ }^{89} \mathrm{Zr}$-mAb 637 was increased in the limbs of 5xFAD (Figure 4.3), but only at 9 months of age. This difference was probably related to different weight distribution in the $5 \mathrm{xFAD}$ mice at this age. In general, the biodistribution of ${ }^{89} \mathrm{Zr}$-mAb 637 was different from ${ }^{89} \mathrm{Zr}$-bapineuzumab (Figure 4.3). However, the clearance rate of both antibodies was the same (Figure 4.3).
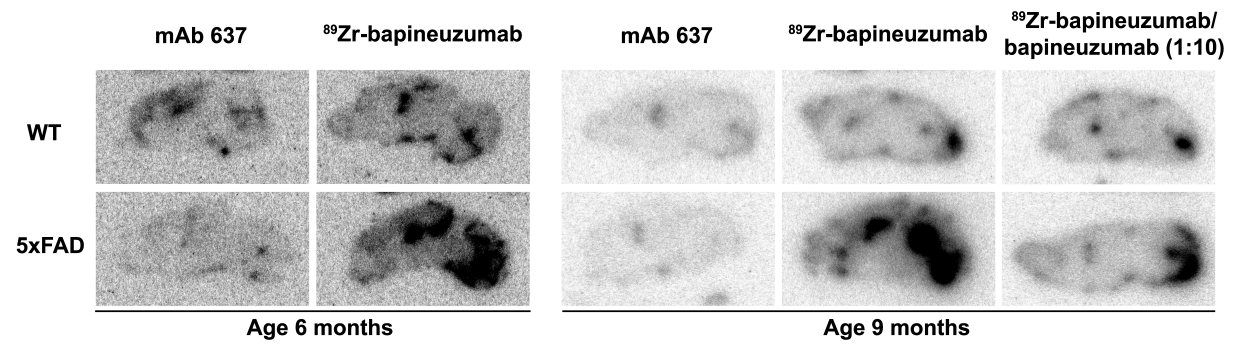

Figure 4.5: Autoradiography showed increased uptake of ${ }^{89} \mathrm{Zr}$-bapineuzumab in the hindbrain. The strongest activity was observed in the hindbrain of 5xFAD animals injected with ${ }^{89} \mathrm{Zr}$-bapineuzumab. This activity was much lower in all animals injected with ${ }^{89} \mathrm{Zr}-\mathrm{mAb} 637$ (isotype control). Blocking bapineuzumab in $5 \mathrm{xFAD}$ mice reduced activity to similar levels as observed in WT animals. Sections of 6-month-old cannot be compared to 9-month-old animals because of inter-assay variations. The hindbrain is orientated to the right in all panels. 


\subsection{4. ${ }^{89}$ ZR-BAPINEUZUMAB CO-LOCALIZED WITH A PLAQUES IN THE BRAIN}

The antibody distribution in the brain was investigated by autoradiography on the brain of 6 and 9 month old animals (Figure 4.5). A clear difference in distribution of radioactivity was observed between the bapineuzumab-injected 5xFAD animals and the control groups: for the 5xFAD animals, most of the activity was observed in the hindbrain, and near the hippocampus region (Figure 4.5).

To investigate the specificity of the in vivo binding of ${ }^{89} \mathrm{Zr}$-labeled antibodies, injected human IgG was immunohistochemically stained in sagittal brain sections. Figure $4.6 \mathrm{~A}$ and $4.6 \mathrm{~B}$ show that plaques stained with ${ }^{89} \mathrm{Zr}$-bapineuzumab, but not with the isotype control ${ }^{89} \mathrm{Zr}-\mathrm{mAb} 637$.

Specificity was further shown by a double immunofluorescent staining for human IgG and $\mathrm{A} \beta$ plaques. ${ }^{89} \mathrm{Zr}$-bapineuzumab colocalized with $\mathrm{A} \beta$ plaques (Figure 4.6C). While antibody $20 \mathrm{C} 2$, which has a preference for oligomeric $\mathrm{A} \beta$, mostly stained the plaque periphery, bapineuzumab was found in the plaque core. 
A
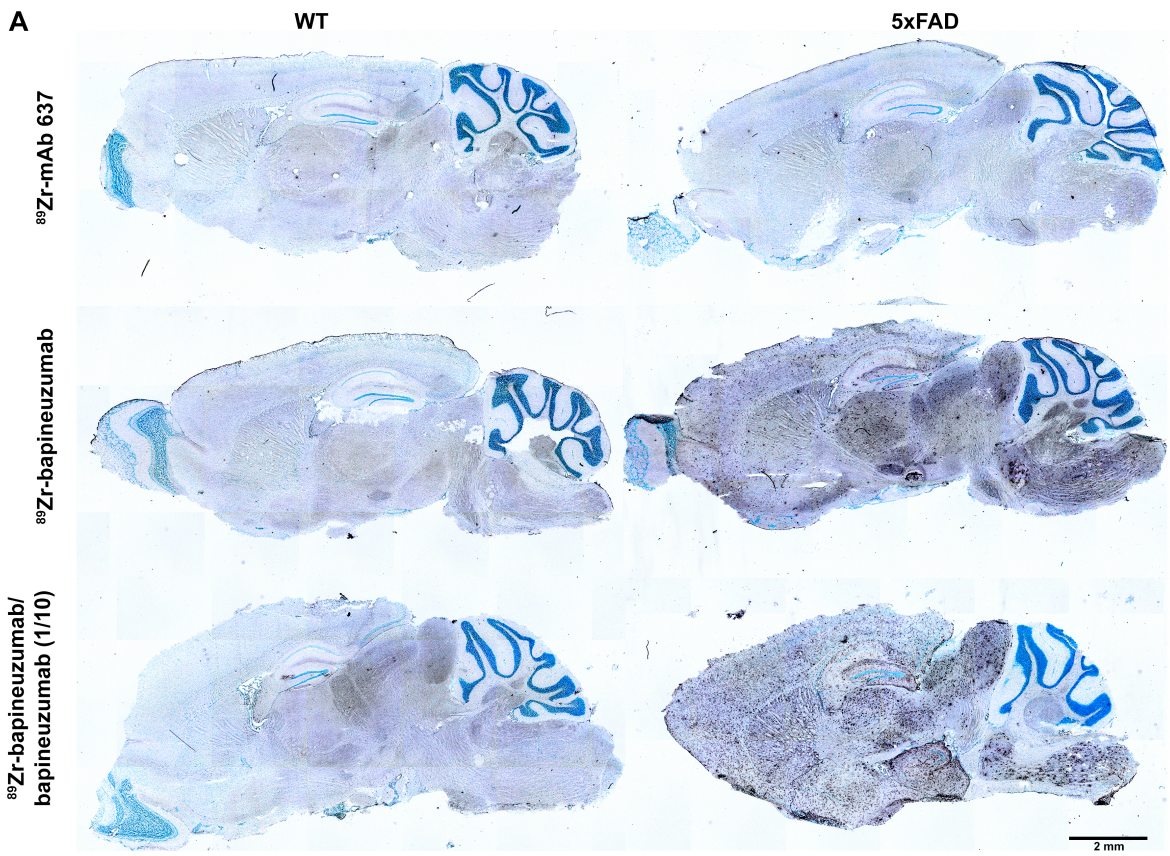

${ }^{89} \mathrm{Zr}-\mathrm{mAb} 637$

B

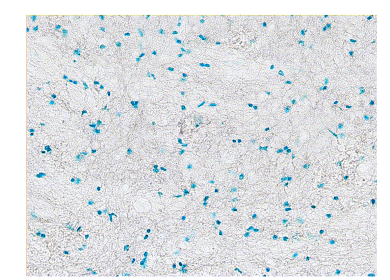

C

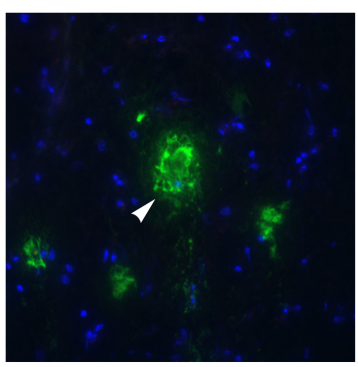

DNA Amyloid $\beta$ Human IgG
${ }^{89} \mathrm{Zr}$-bapineuzumab
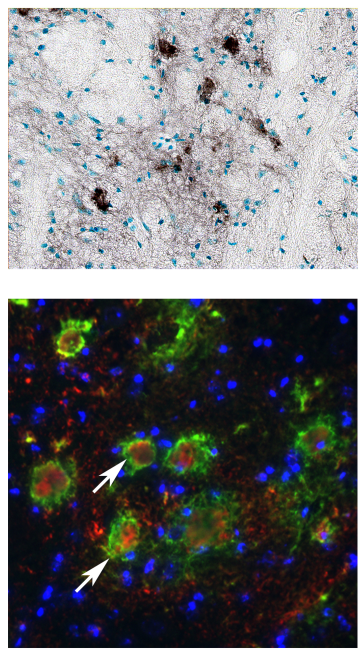

${ }^{89} \mathrm{Zr}$-bapineuzumab/ bapineuzumab (1/10)
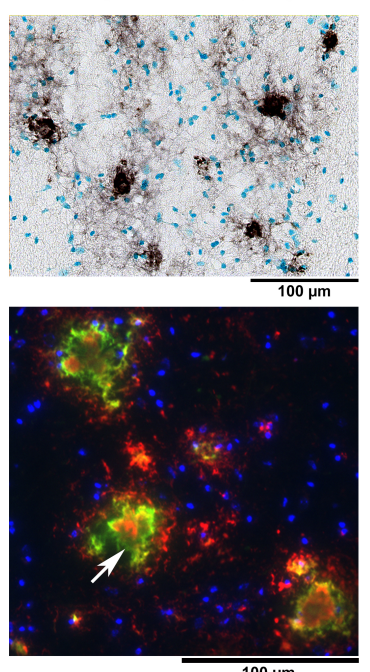

Figure 4.6: In vivo injected ${ }^{89} \mathrm{Zr}$-bapineuzumab is present in $\mathrm{A}$ plaques. (A) Immunohistochemistry for human IgG (anti-human IgG) with a methyl green counterstain on brains of 9-month old mice showed a diffuse staining predominantly in the mid- and hindbrain. In $5 x F A D$ animals injected with ${ }^{89} \mathrm{Zr}$-bapineuzumab, also plaques were clearly stained. In all groups, there is clearly less human IgG present in the hippocampus. (B) High power photomicrographs of the $5 \mathrm{xFAD}$ brain stem showed that plaques were immunoreactive for human IgG in animals treated with ${ }^{89} \mathrm{Zr}$-bapineuzumab. This immunoreactivity could not be found in $5 \mathrm{xFAD}$ animals treated with mAb 637. (C) Double labeling of plaques in the brain stem with anti-Aß 20C2 (green) and antihuman IgG (red) showed colocalization. Cell nuclei were stained with Hoechst (blue). ${ }^{89} \mathrm{Zr}$-bapineuzumab was detected in the plaque core (arrows), while 20C2 staining was predominantly in the plaque periphery. More intense staining of plaques was observed when bapineuzumab was blocked with excess unlabeled bapineuzumab. The isotype control did not show colocalization with plaques (arrow head). 


\subsection{DISCUSSION}

This study shows that immuno-PET with ${ }^{89} \mathrm{Zr}$-bapineuzumab can be used for specific and sensitive detection of $A \beta$ pathology in the brain. As early as at 3 months of age, brain uptake of ${ }^{89} \mathrm{Zr}$-bapineuzumab was increased in 5xFAD mice. The observed agerelated increase of ${ }^{89} \mathrm{Zr}$-bapineuzumab accumulation in the brain in the biodistribution analysis clearly followed the documented $A \beta$ build-up with age in this animal model [11]. Interestingly, ${ }^{89} \mathrm{Zr}$-bapineuzumab also showed specific uptake in the cervical lymph nodes of $5 x F A D$. Since much of the CSF drains through this route, $A \beta$ or IgG-A $\beta$ complexes are likely to accumulate in these lymph nodes [22].

The specificity of the signal was demonstrated by using a radiolabeled isotype control mAb, which showed no difference in brain accumulation between $5 \mathrm{xFAD}$ and WT mice. This suggests that ${ }^{89} \mathrm{Zr}$-bapineuzumab accumulation is specific to $\mathrm{A} \beta$ pathology and not due to an increased permeability of the BBB. In this context it is important to mention that the BBB allows controlled entry of IgG in the brain under physiological conditions [5]. Even though the amount of penetrated antibody is low, the good signal-to-noise ratio still makes it possible to detect a specific signal. Blocking with cold bapineuzumab reduced brain uptake of ${ }^{89} \mathrm{Zr}$-bapineuzumab to baseline values, providing further evidence for the specificity of immuno-PET using ${ }^{89} \mathrm{Zr}$-bapineuzumab.

The long half-life of ${ }^{89} \mathrm{Zr}$ allowed us to assess antibody kinetics over a period of 9 days. Three days after ${ }^{89} \mathrm{Zr}$-bapineuzumab injection, significant uptake of the antibody was detected in the brain. This uptake increased further during the subsequent days, showing accumulation of antibody over time.

Recently, similar results were obtained by Sehlin and colleagues., where an anti-A $\beta$ antibody against protofibrils was used for in vivo PET imaging [23]. In contrast to our study, Sehlin et al. made use of a bispecific antibody targeting the transferrin-receptor in order to increase antibody uptake in the brain. We show that it possible to use antibodies for CNS imaging without modifications besides the radiolabel. Both our results prove that antibodies allow for specific in vivo imaging in the CNS.

Rojas et al. compared current amyloid PET tracers in 5xFAD and WT animals. They were also able to discriminate between $5 \mathrm{xFAD}$ and WT animals with a difference of $14.5 \%$ and $21 \%$ in activity between 11-months-old 5xFAD and WT animals using florbetapir and $\mathrm{PiB}$ respectively. Here we show that immuno-PET with ${ }^{89} \mathrm{Zr}$-bapineuzumab offers a significant leap in sensitivity. Over a $50 \%$ difference of ${ }^{89} \mathrm{Zr}$-bapineuzumab uptake was present between 9-month-old 5xFAD and WT animals [24]. These results show that despite the lower penetration of bapineuzumab compared to traditional amyloid tracers, a higher sensitivity can be reached, likely because of very high-affinity binding of bapineuzumab to $\mathrm{A} \beta$.

A limitation of this study is the difference in biodistribution of the isotype control ${ }^{89} \mathrm{Zr}$-mAb 637 and ${ }^{89} \mathrm{Zr}$-bapineuzumab. However, the clearance rate of both antibodies from the blood was the same. We confirmed the age-related increase of plaque load in the 5xFAD model by immunohistochemistry with bapineuzumab. This increase was also observed in the biodistribution analysis with ${ }^{89} \mathrm{Zr}$-bapineuzumab. However, no agerelated increase of ${ }^{89} \mathrm{Zr}$-bapineuzumab uptake was observed in the corresponding in vivo PET scans. This might be related to partial volume effects in the PET scans, caused by accumulation of ${ }^{89} \mathrm{Zr}$ in the skull, making quantification of the brain more difficult. 
This seems to be especially a problem with rodents, and less of an issue when imaging with ${ }^{89} \mathrm{Zr}$ in humans [25]. Additionally, while a relatively high dose of radioactivity was used in our mice, new advances in clinical PET scanners allow for the use of a significant lower dose in humans [26].

Our results show that it is possible to use an antibody to detect $A \beta$ pathology in the CNS in vivo. With ${ }^{89} \mathrm{Zr}$-immuno-PET it is possible to image specific antibody binding to $A \beta$, even in the early disease stage, when $A \beta$ load is low. Anti- $A \beta$ immuno-PET could potentially be useful as an inclusion criterion for passive anti- $A \beta$ immunotherapy trials. It may also be a powerful tool for the selection of the optimal antibody format (e.g. intact antibody or antibody fragment), and for optimization of the antibody dosing schedule in anti-A $\beta$ immunotherapy studies. Furthermore, if new therapeutic antibodies become available [27], this technique will allow such antibodies to be used as a theranostic tool to screen patients at baseline and during treatment, although attention has to be paid to radiation safety when multiple scans are undertaken. Finally, antibodies might be useful to develop new tracers for other neurodegenerative disorders by targeting for example $\alpha$-synuclein in Parkinson's disease, or tau tangles in AD or other tauopathies. 


\section{REFERENCES}

[1] Vosjan MJWD, Perk LR, Visser GWM, Budde M, Jurek P, Kiefer GE, et al. Conjugation and radiolabeling of monoclonal antibodies with zirconium-89 for PET imaging using the bifunctional chelate p-isothiocyanatobenzyl-desferrioxamine [Journal Article]. Nat Protocols. 2010;5(4):739-743. Available from: http://dx.doi.org/ 10.1038/nprot.2010.13.

[2] Vugts DJ, van Dongen GAMS. 89Zr-labeled compounds for PET imaging guided personalized therapy [Journal Article]. Drug Discovery Today: Technologies. 2011;8(2-4):e53-e61. Available from: http://www.sciencedirect.com/ science/article/pii/S1740674911000370.

[3] Martinez-Martinez P, Molenaar PC, Losen M, Stevens J, Baets MHD, Szoke A, et al. Autoantibodies to neurotransmitter receptors and ion channels: from neuromuscular to neuropsychiatric disorders [Journal Article]. Frontiers in Genetics. 2013;4:181. Available from: http://www.ncbi.nlm.nih.gov/pmc/ articles/PMC3778371/.

[4] Cutler RWP, Watters GV, Hammerstad JP. The origin and turnover rates of cerebrospinal fluid albumin and gamma-globulin in man [Journal Article]. Journal of the Neurological Sciences. 1970;10(3):259-268. Available from: http://www . sciencedirect.com/science/article/pii/0022510X70901541.

[5] Poduslo JF, Curran GL, Berg CT. Macromolecular permeability across the bloodnerve and blood-brain barriers [Journal Article]. Proceedings of the National Academy of Sciences of the United States of America. 1994;91(12):5705-5709. Available from: http://www.ncbi.nlm.nih.gov/pmc/articles/PMC44065/.

[6] Black RS, Sperling RA, Safirstein B, Motter RN, Pallay A, Nichols A, et al. A single ascending dose study of bapineuzumab in patients with Alzheimer disease [Journal Article]. Alzheimer disease and associated disorders. 2010;24(2):198.

[7] Miles LA, Crespi GAN, Doughty L, Parker MW. Bapineuzumab captures the Nterminus of the Alzheimer's disease amyloid-beta peptide in a helical conformation [Journal Article]. Sci Rep. 2013;3. Available from: http://dx.doi.org/10.1038/ srep01302.

[8] Salloway S, Sperling R, Fox NC, Blennow K, Klunk W, Raskind M, et al. Two Phase 3 Trials of Bapineuzumab in Mild-to-Moderate Alzheimer's Disease [Journal Article]. New England Journal of Medicine. 2014;370(4):322-333. Available from: http:// www.nejm.org/doi/full/10.1056/NEJMoa1304839.

[9] Blennow K, Hampel H, Zetterberg H. Biomarkers in Amyloid- $\beta$ Immunotherapy Trials in Alzheimer/'s Disease [Journal Article]. Neuropsychopharmacology. 2014;39(1):189-201. Available from: http://dx.doi.org/10.1038/npp. 2013. 154. 
[10] Carrillo MC, Brashear HR, Logovinsky V, Ryan JM, Feldman HH, Siemers ER, et al. Can we prevent Alzheimer's disease? Secondary "prevention" trials in Alzheimer's disease [Journal Article]. Alzheimer's \& Dementia. 2013;9(2):123131.el. Available from: http://www.sciencedirect.com/science/article/ $\mathrm{pii/S1552526012025873.}$

[11] Oakley H, Cole SL, Logan S, Maus E, Shao P, Craft J, et al. Intraneuronal ßAmyloid Aggregates, Neurodegeneration, and Neuron Loss in Transgenic Mice with Five Familial Alzheimer's Disease Mutations: Potential Factors in Amyloid Plaque Formation [Journal Article]. The Journal of Neuroscience. 2006;26(40):10129_ 10140. Available from: http://www.jneurosci.org/content/26/40/10129. abstract.

[12] Jia A, Tsurushita N, Vasquez M. Anti-abeta antibodies. Google Patents; 2002. US Patent App. 10/487,322.

[13] van der Neut Kolfschoten M, Schuurman J, Losen M, Bleeker WK, MartinezMartinez P, Vermeulen E, et al. Anti-inflammatory activity of human IgG4 antibodies by dynamic Fab arm exchange [Journal Article]. Science. 2007;317(5844):1554-7. Available from: http://www.ncbi.nlm.nih.gov/pubmed/17872445.

[14] Verel I, Visser GWM, Boellaard R, Stigter-van Walsum M, Snow GB, van Dongen GAMS. 89Zr Immuno-PET: Comprehensive Procedures for the Production of 89ZrLabeled Monoclonal Antibodies [Journal Article]. Journal of Nuclear Medicine. 2003;44(8):1271-1281. Available from: http://jnm. snmjournals . org/content/ 44/8/1271. abstract.

[15] Alakurtti K, Johansson JJ, Joutsa J, Laine M, Backman L, Nyberg L, et al. Long-term test-retest reliability of striatal and extrastriatal dopamine D2/3 receptor binding: study with 11C raclopride and high-resolution PET [Journal Article]. J Cereb Blood Flow Metab. 2015;Available from: http://dx.doi.org/10.1038/jcbfm. 2015. 53.

[16] Vállez Garcia D, Casteels C, Schwarz AJ, Dierckx RAJO, Koole M, Doorduin J. A Standardized Method for the Construction of Tracer Specific PET and SPECT Rat Brain Templates: Validation and Implementation of a Toolbox [Journal Article]. PLoS ONE. 2015;10(3):e0122363. Available from: http://dx.doi.org/10.1371\% 2Fjournal pone. 0122363.

[17] Granton PV, Dubois L, van Elmpt W, van Hoof SJ, Lieuwes NG, De Ruysscher D, et al. A Longitudinal Evaluation of Partial Lung Irradiation in Mice by Using a Dedicated Image-Guided Small Animal Irradiator [Journal Article]. International Journal of Radiation Oncology*Biology*Physics. 2014;90(3):696-704. Available from: http: //www.sciencedirect.com/science/article/pii/S0360301614034713.

[18] Abou DS, Ku T, Smith-Jones PM. In vivo biodistribution and accumulation of 89Zr in mice [Journal Article]. Nuclear Medicine and Biology. 2011;38(5):675681. Available from: http://www.sciencedirect.com/science/article/pii/ S0969805110005214. 
[19] Mencarelli C, Bode GH, Losen M, Kulharia M, Molenaar PC, Veerhuis R, et al. Goodpasture antigen-binding protein/ceramide transporter binds to human serum amyloid P-component and is present in brain amyloid plaques [Journal Article]. The Journal of biological chemistry. 2012;287(18):14897-911. Available from: http://www.ncbi.nlm.nih.gov/pubmed/22396542.

[20] Edelstein AD, Tsuchida MA, Amodaj N, Pinkard H, Vale RD, Stuurman N. Advanced methods of microscope control using $\mu$ Manager software [Journal Article]. Journal of Biological Methods. 2014;1(2):e10.

[21] Preibisch S, Saalfeld S, Tomancak P. Globally optimal stitching of tiled 3D microscopic image acquisitions [Journal Article]. Bioinformatics. 2009;25(11):1463-5. Available from: http://www.ncbi.nlm.nih.gov/pubmed/19346324.

[22] Weller RO, Galea I, Carare RO, Minagar A. Pathophysiology of the lymphatic drainage of the central nervous system: Implications for pathogenesis and therapy of multiple sclerosis [Journal Article]. Pathophysiology. 2010;17(4):295306. Available from: http://www.sciencedirect.com/science/article/pii/ S0928468009001175.

[23] Sehlin D, Fang XT, Cato L, Antoni G, Lannfelt L, Syvanen S. Antibody-based PET imaging of amyloid beta in mouse models of Alzheimer/'s disease [Journal Article]. Nat Commun. 2016;7. Available from: http://dx.doi.org/10.1038/ ncomms 10759 .

[24] Rojas S, Herance JR, Gispert JD, Abad S, Torrent l, Jiménez X, et al. In vivo evaluation of amyloid deposition and brain glucose metabolism of 5XFAD mice using positron emission tomography [Journal Article]. Neurobiology of Aging. 2013;34(7):1790-1798. Available from: http: //www . neurobiologyofaging . org/ article/S0197-4580(12)00660-4/abstract.

[25] Fissers J, Waldron AM, De Vijlder T, Van Broeck B, Pemberton DJ, Mercken M, et al. Synthesis and Evaluation of a Zr-89-Labeled Monoclonal Antibody for ImmunoPET Imaging of Amyloid- $ß$ Deposition in the Brain [Journal Article]. Molecular Imaging and Biology. 2016;p. 1-8.

[26] van Dongen GA, Vosjan MJ. Immuno-positron emission tomography: shedding light on clinical antibody therapy [Journal Article]. Cancer biotherapy \& radiopharmaceuticals. 2010;25(4):375-385.

[27] Idec B. Single Ascending Dose Study of BIIB037 in Participants With Alzheimer's Disease. www.clinicaltrials.gov; 2013. 



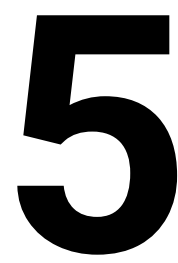

\section{A COMPARISON OF THREE ANTI-AMYLOID ANTIBODIES WITH IMMUNO-PET}

Jo A.A. Stevens, Pilar Martinez-Martinez, Danielle J. Vugts, Thijs E. Nell, Simone M. Crivelli, Guus A. van Dongen, Mario Losen In preparation 


\section{ABSTRACT}

The amyloid cascade hypothesis is the leading hypothesis to explain Alzheimer's disease (AD) pathogenesis. It states that there are different forms of $A \beta$, of which the oligomeric form is the most toxic. In the last years, several immunotherapies have been developed to remove $A \beta$ from the brain, but so far, results have been largely disappointing. In an effort to better understand the behavior of these $A \beta$ antibodies in vivo, three anti-A $\beta$ antibodies targeting monomers (solanezumab), oligomers (20C2), and fibrils (bapineuzumab) were radiolabeled with ${ }^{89} \mathrm{Zr}$, a long-lived positron emitter. Labeled antibodies were administered to 5xFAD mice of different ages and in vivo PET scans were performed. In addition, a detailed biodistribution study was performed. Results obtained from these assays were compared with ex vivo A $\beta$ quantification. Our results showed that ${ }^{89} \mathrm{Zr}$-bapineuzumab accumulated in an age-related manner in the brain, correlating with plaque load. While oligomers accumulated as animals age ex vivo, in vivo ${ }^{89} \mathrm{Zr}$-20C2 only accumulated in $\mathrm{AD}$ animals at later ages. In the early disease stage, ${ }^{89} \mathrm{Zr}$-20C2 was actually removed faster from $\mathrm{AD}$ animals. Monomeric $\mathrm{A} \beta$ remained constant as animals age, and there was also no ${ }^{89} \mathrm{Zr}$-solanezumab accumulation in vivo. Interestingly, ${ }^{89} \mathrm{Zr}$-solanezumab accumulated in adipose tissue in 5xFAD animals and this correlated with monomeric plaque load in the brain. These results prove that it is important to further study in vivo antibody properties in order to further improve and refine antibody targets. 


\subsection{INTRODUCTION}

Amyloid- $\beta(A \beta)$ is one of the defining hallmarks of Alzheimer's disease $(A D)$ and has been one of the main targets of new experimental AD therapies. Most of these therapies focus on lowering $A \beta$ production (eg. gamma- and beta-secretase inhibitors) or on increasing $A \beta$ clearance from the brain [1]. The latter has been attempted by means of active and passive immunization against $A \beta$.

Several passive immunization strategies, where $A \beta$ antibodies are administered to the patient, have been or are being conducted. The first passive immunotherapy phase II clinical trial, conducted by Johnson and Johnson, made use of the antibody bapineuzumab. Bapineuzumab, the humanized variant of the original mouse antibody 3D6, targets the $\mathrm{N}$-terminus region of $\mathrm{A} \beta$ (amino acids 1-5) [2]. Bapineuzumab has been shown to bind both soluble and fibrillary amyloid [3]. While the phase II trial did not meet clinical end-points, the antibody was progressed to phase III based on a subset analysis showing some possible benefit for APOE4 non-carriers [4]. The phase III trial was concluded with negative primary endpoints in the summer of 2013 [5]. Recent results did indicate that bapineuzumab lowered fibrillary $\mathrm{A} \beta$ load as assessed by ${ }^{11} \mathrm{C}$ PiB-PET imaging [6].

Several reasons have been postulated for this failure. A first reason is that during the trial, dosage had to be lowered 2 -fold because patients experienced microbleeds because of treatment [6]. It might therefore be the case that antibody dosing was too low to be effective. Secondly, it is estimated that up to $20 \%$ of the patients were misdiagnosed and did not suffer from $\mathrm{AD}$ at all [7]. The net result being that the power of the trial is severely lowered and a possible beneficial effect could not be picked up. Third, it has been suggested that treatment started too late and patients need to start treatment when the disease is still in its beginning stages [8]. A final hypothesis might be that bapineuzumab targeted the wrong $A \beta$ species, and another species such as monomeric or oligomeric $A \beta$ might have showed more efficacy [6].

Solanezumab is another antibody that has been tested in large phase III clinical trials. Solanezumab, mouse equivalent antibody m266, binds to the midregion of A $\beta$ (amino acids 16-26) [9]. Furthermore, it has been shown to have a high affinity for monomeric amyloid, presumable because the epitope is masked in fibrillary or oligomeric assemblies of $A \beta$ [9]. Primary endpoints of this antibody in the phase III trial were not met. There was a small effect in patients with mild AD, and the antibody is now begin continued in a prevention trial [10]. Compared to bapineuzumab, this antibody did not seem to cause microbleeds in patients [11]. Another difference is its presumed mechanism of action. Solanezumab is assumed to not enter the brain, but instead lowers $A \beta$ in the brain by sequestering free $A \beta$ in the blood stream, and preventing this $A \beta$ from reentering in the brain, thereby causing a net efflux of amyloid. This mechanism is also known as the peripheral sink hypothesis [12].

While the clinical trials of these antibodies have yielded disappointing results, it is still important to further characterize these antibodies so more can be understood as to why these antibodies failed clinical trials. Recently, we proved that immuno-PET can be a valuable tool to gain more understanding about $\mathrm{A} \beta$ antibody binding and distribution in vivo. In that study, bapineuzumab was conjugated to the long-lived positron emitter 89-zirconium $\left({ }^{89} \mathrm{Zr}\right)$ and injected in an $\mathrm{AD}$ mouse model $(5 \mathrm{xFAD})$ at various disease 
stadia. The 5xFAD model is rapid disease onset mouse model containing five familial $\mathrm{AD}$ mutations (3 in APP and 2 PSEN1) [13]. Immuno-PET with bapineuzumab allowed detection of $\mathrm{AD}$ pathology at the young age of 3 months [14]. At this age, the first plaques appear in the 5xFAD model [13].

In this paper, we expand on these results by comparing bapineuzumab against solanezumab using immuno-PET in the $5 \times$ FAD mouse model. Additionally, an anti-A $\beta$ antibody targeting oligomeric forms of $A \beta$ was also investigated. The amyloid cascade hypothesis posits amyloid accumulation as the central cause of AD [15]. Over the years, the hypothesis has been updated and fine-tuned, and it is now believed that oligomeric assemblies of $\mathrm{A} \beta$ are the most cytotoxic and the main cause of $\mathrm{AD}$ [16]. Surprisingly, no immunotherapies focusing on targeting oligomeric forms of $A \beta$ have been tested yet in pre-clinical or clinical trials. Antibody 20C2 is an antibody that targets soluble oligomers of $A \beta$ [17]. It has been extensively characterized and binds to a conformational epitope [18]. To gain further insight into the targeting mechanisms of these three different antibodies, we compared them in the $5 \mathrm{xFAD}$ model at different ages. Since monomeric and oligomeric forms of $A \beta$ originate first, we hypothesized that solanezumab and $20 \mathrm{C} 2$ would allow for more sensitive and early detection than bapineuzumab, which binds mostly to plaques. Our hypothesis was not confirmed, due to the fact that antibodies binding soluble forms of $A \beta$ also get cleared faster. Furthermore, the antioligomeric antibody 20C2 exhibited different distributional patterns in different stages of the disease. These results might have important implications for ongoing and planned clinical trials.

\subsection{METHODS}

\subsubsection{ANIMALS}

BS/SJL wild type (WT) and 5xFAD animals were purchased from Jackson Laboratories (stock 6554). Mice were bred hemizygously using F3 and F4 WT mice. Mice were housed socially with ad libitum access to food and water on a 12-hour day-night cycle. For all experiments, female mice were used of the ages 3, 6, or 9 months. All experiments were approved by the Animal Welfare Committee of Maastricht University and followed the law and guidelines of the Netherlands.

Table 5.1: Number of animals per experimental group

\begin{tabular}{llll}
\hline Age (months) & ${ }^{89}$ Zr-bapineuzumab & ${ }^{89}$ Zr-solanezumab & ${ }^{89}$ Zr-20C2 \\
\hline & WT & 5 xFAD & WT \\
3 & 5 & 5 & 5 \\
6 & 5 & 5 & 7 \\
9 & 4 & 4 & 4 \\
\hline
\end{tabular}

\subsubsection{ANTIBODY PRODUCTION AND LABELING}

Antibodies were cloned and produced as described [14]. Briefly, antibody sequences of bapineuzumab [19], solanezumab [20] and 20C2 [21] were synthesized de novo by 
GeneArt (Invitrogen) and cloned into pFuse expression vectors (Invivogen). Plasmids were produced at large scale in a New Brunswick Bioflo 110 bioreactor and purified on an AKTA explorer system equipped with a CIM DEAE column.

HEK-293 F cells were transfected with linear $22 \mathrm{kDa}$ PEI (Polysciences) in Nunc cell factories in Freestyle 293 medium. Four hours after transfection valproic acid (Sigma Aldrich) was added to a concentration of $4 \mathrm{mM}$. After 10 days, supernatant was filtered through a $0.45 \mu \mathrm{m}$ membrane and concentrated using an artificial kidney (Fresenius Medical Care). Antibody was subsequently purified using a protein A column (GE Healthcare) followed by a buffer exchange to PBS using a Sephadex column (GE Healthcare).

Labeling with zirkonium-89 was carried out as described.

A total of $100 \mu \mathrm{g}$ antibody dissolved in $200 \mu \mathrm{L}$ saline was injected intraperitoneally in animals under isoflurane anesthesia. Average injected activity was 3.47 MBq. Animals numbers per group are listed in Table 5.1.

\subsubsection{IMAGING}

In vivo PET images were acquired as described [14]. Animals were placed supine in the center field of view (FOV) of a Siemens MicroPET Focus 120 (Siemens Healthcare) with an axial FOV of $7.6 \mathrm{~cm}$ and a resolution of $1.3 \mathrm{~mm}$. Scans were acquired for a total of 30 minutes while animals were anesthetized using isoflurane $\left(2.5 \%\right.$ in $\left.\mathrm{O}_{2}\right)$. Body temperature was maintained using an electrical heating pad. Data were acquired with correction for dead time, random coincidences and decay. Images were reconstructed using OSEM2D and PET data were analyzed using Carimas 2.9 [22]. For analysis, the head was first coregistered using normalized cross correlation to a CT template scan that was acquired as described [23]. Manual realignment was performed if the automatic coregistration algorithm failed. Next, a volume of interest (VOI) of $25 \mu \mathrm{l}$ was selected in the CT template and mean Bq/mL was calculated of the VOI (see Fig. 1). The VOI was chosen small to minimize partial volume effects caused by high activity of the surrounding tissues such as the bone [24]. The standardized uptake value (SUV) was calculated by correcting the mean activity of the VOI (in $\mathrm{Bq} / \mathrm{ml}$ ) for body weight (in $\mathrm{g}$ ), injected dose $(\mathrm{Bq})$ and decay .

\subsubsection{BIODISTRIBUTION}

After sacrificing animals by cervical dislocation, biodistribution was performed as described [14]. Briefly, one brain hemisphere was frozen with liquid nitrogen and stored at -80 . The other hemisphere was macrodissected into hindbrain (with cerebellum and brain stem), bulbus, hippocampus and cortex. The following tissues and organs were dissected, and radioactivity was measured: blood, salivary glands, cervical lymph nodes, skin, abdominal adipose tissue, eyes, spleen, intestines, liver, stomach, bladder, kidneys, lungs and heart. Remaining body parts were divided into limbs, head, and trunk. Radioactivity was measured using a 2470 Wizard $^{2}$ gamma counter (Perkin Elmer) for a total time of 2 minutes. All results were corrected for decay and expressed as a percentage of total injected dose $(\mathrm{Bq})$ per gram of tissue $(\% \mathrm{ID} / \mathrm{g})$. 


\subsubsection{IMMUNOSTAINING AND MICROSCOPY}

Frozen hemispheres were cut sagittal into $16 \mu \mathrm{m}$ sections on a Leica cryostat at $17{ }^{\circ} \mathrm{C}$. Sections were post-fixated with $4 \%$ paraformaldehyde. For analysis of plaque load, sections were incubated overnight with biotinylated bapineuzumab (1:4000), solanezumab (1:2000) or 20C2 (1:4000). Sections were washed and incubated with ABC Kit (Vectastain). After washing, sections were incubated with horseradish peroxidase, dehydrated and coverslipped with DPX (Klinipath).

Photomicrographs were acquired using $\mu$ Manager software [25] on a IX-81 inverted microscope (Olympus). Images were background corrected and stitched using a plug-in [26] of ImageJ 1.49 software (www.imagej.nih.gov/ij/). Next, regions-of-interest (ROIs) were delineated consisting of cortex, subiculum, hippocampus, midbrain, thalamus and striatum. A median filter was applied to all images, and a threshold was determined. Images were binarized and plaque load was analyzed using the particle analyzer tool in ImageJ. All processing steps were identical for each antibody staining.

\subsubsection{STATISTICAL ANALYZES}

Statistical analyzes were performed with SPSS 23 (IBM). In vivo PET data and biodistribtion was calculated with a 3-way ANOVA with age, antibody and genotype as factors. The radioactivity of level of the fat tissue was compared using a 2-way ANOVA with age and genotype as factor for each antibody, because no data was available for bapineuzumab for 9 month old animals. For correlations, Pearson correlation was calculated. A p-value of 0.05 was considered significant. 


\subsection{Results}

Figure 5.1: A qualitative and quantitative comparison of bapineuzumab, solanezumab and 20C2. A) Representative images of 9 months old $5 x F A D$ animals show that staining with bapineuzumab and 20C2 gives comparable patterns. Solanezumab staining is characterized by an increased background, diffuse staining pattern. In addition, plaques are much smaller in comparison to stained plaques with bapineuzumab and 20C2. B) Plaque load was assessed by measuring the density in the brain at three different ages. While initially, at the age of 9 months, bapineuzumab and 20C2 showed a significantly increased plaque density compared to solaneuzumab $(\mathrm{p}<0.001)$. Plaque density as measured with solanezumab remained constant over time. C) Staining with solanezumab shows significant more detection of small-sized plaques than staining with bapineuzumab or 20C2 $(\mathrm{p}<0.01)$. In addition, while bapineuzumab and 20C2 show an increase in amount of plaques over time, this was not the case with solanezumab. D) Almost no medium sized plaques are labeled with solanezumab. Bapineuzumab and 20C2 show an age-related increase in medium-sized plaque numbers. No differences were present between bapineuzumab and 20C2 E) Almost no large sized plaques are labeled with solanezumab. Bapineuzumab and $20 \mathrm{C} 2$ show an age-related increase, and 20C2 showed a trend to label larger sized plaques at 9 months of age $(\mathrm{p}=0.065)$. $(\mathrm{n}=2-7$ per group, error bars denote SEM).
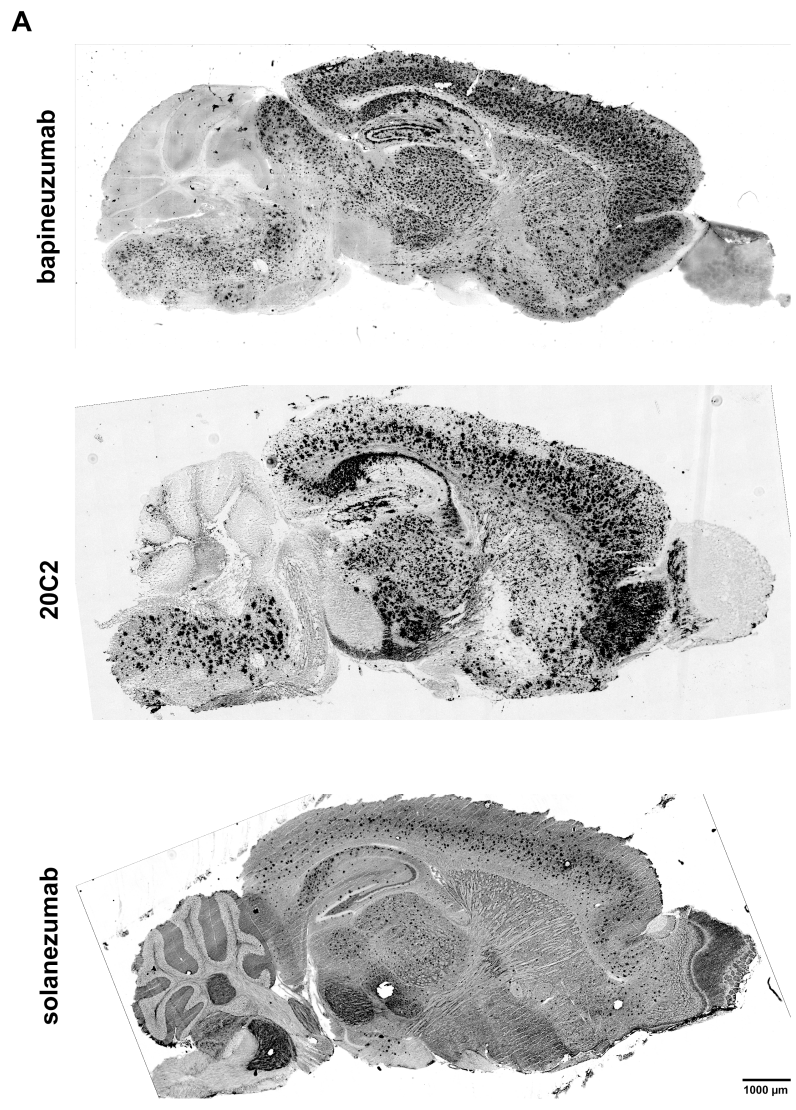

B
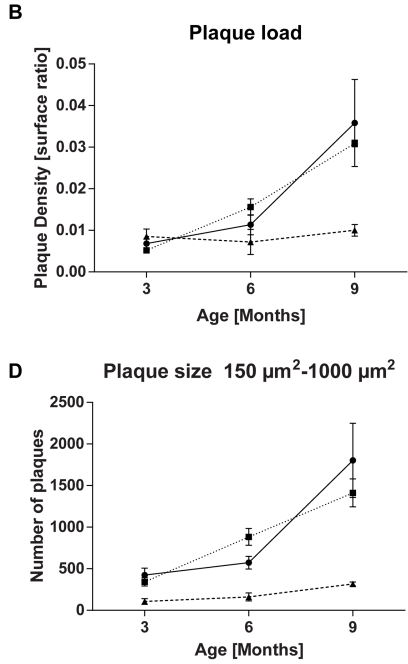

- Bapineuzumab -...- Solanezumab
C

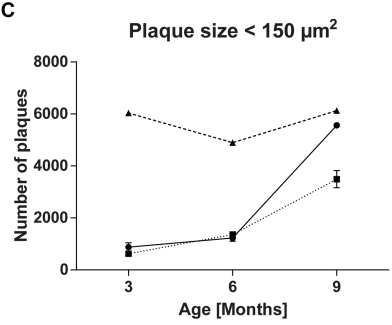

E

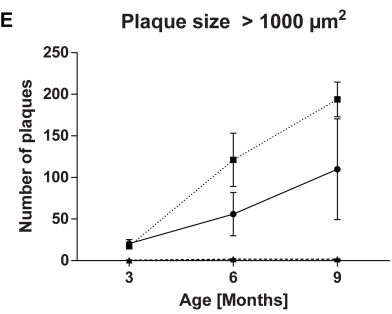




\subsubsection{SOLUBLE A $\beta$ DOES NOT INCREASE IN BRAIN OVER TIME}

Three anti-A $\beta$ antibodies, targeting soluble $A \beta$, oligomers, and fibrils, were compared in vivo and ex vivo on their binding properties in the $5 \mathrm{xFAD}$ mouse brain at different ages. First, plaque load and size was assessed at the ages of 3, 6, and 9 months. An immunohistochemical staining was performed using solanezumab (soluble $A \beta$ ), 20C2 ( $\mathrm{A} \beta$ oligomers), and bapineuzumab ( $\mathrm{A} \beta$ fibrils). Qualitative comparison showed that staining with solanezumab gave a very diffuse staining, and only lightly labeled plaques (see Figure 5.1A). Plaques looked also much smaller when compared to the other antibodies. 20C2 and bapineuzumab gave a very comparable staining pattern. All three antibodies showed the most abundant plaque load in the subiculum and the cortex.

Quantitatively, we could detect an age-related increase in plaque load with bapineuzumab and 20C2. Solanezumab was different in this regard, in that it showed no increase at all $(\mathrm{p}<0.01)$. The difference between these three antibodies became further apparent when we compared the amount of plaques that they detected at the different ages. Solanezumab detected mostly small plaques $\left(<150 \mu \mathrm{m}^{2}\right)$, and the amount of plaques detected remained constant over time $(\mathrm{p}=0.847)$. In addition, solanezumab detected very few to none medium sized $\left(150-1000 \mu \mathrm{m}^{2}\right)$ and large plaques $\left(>1000 \mu \mathrm{m}^{2}\right)$ (see Figure 5.1). No significant differences were found between $20 \mathrm{C} 2$ and bapineuzumab on plaque load or plaque sizes (see Figure 5.1), although 20C2 did show a trend to label more large-sized plaques than bapineuzumab $(\mathrm{p}=0.065)$.

\subsubsection{ONLY ${ }^{89}$ ZR-BAPINEUZUMAB ALLOWS FOR IN VIVO DIAGNOSIS}

To further compare the binding differences between these antibodies in vivo experiments were performed. Previously, we have shown that immuno-PET is a suitable technique for investigating antibody binding in vivo [14]. For immuno-PET experiments, antibodies were first labeled with ${ }^{89} \mathrm{Zr}$. Next, antibodies were administered to $5 \mathrm{xFAD}$ animals and WT controls at different ages. Five days after administration, in vivo PET scans were performed followed by biodistribution.

As previously reported, quantification of PET scans showed that there was increased ${ }^{89} \mathrm{Zr}$-bapineuzumab uptake in $5 \times \mathrm{XAD}$ animals at all ages $(\mathrm{p}<0.01)$, but uptake did not increase as animals aged. In contrast to ${ }^{89} \mathrm{Zr}$-bapineuzumab uptake, retention of ${ }^{89} \mathrm{Zr}$-solanezumab ( $\left.\mathrm{p}=0.99\right)$ or ${ }^{89} \mathrm{Zr}-20 \mathrm{C} 2(\mathrm{p}=0.39)$ was not increased in $5 \mathrm{xFAD}$ animals compared to WT animals at any age (see Figure 5.2A). Uptake of ${ }^{89} \mathrm{Zr}-20 \mathrm{C} 2$ in both $5 \mathrm{xFAD}$ and WT animals was comparable to ${ }^{89} \mathrm{Zr}$-bapineuzumab uptake in WT animals. ${ }^{89} \mathrm{Zr}$ solanezumab uptake however was significantly lower in both WT and 5xFAD animals at every age when compared to the other two antibodies $(\mathrm{p}<0.01)$. 

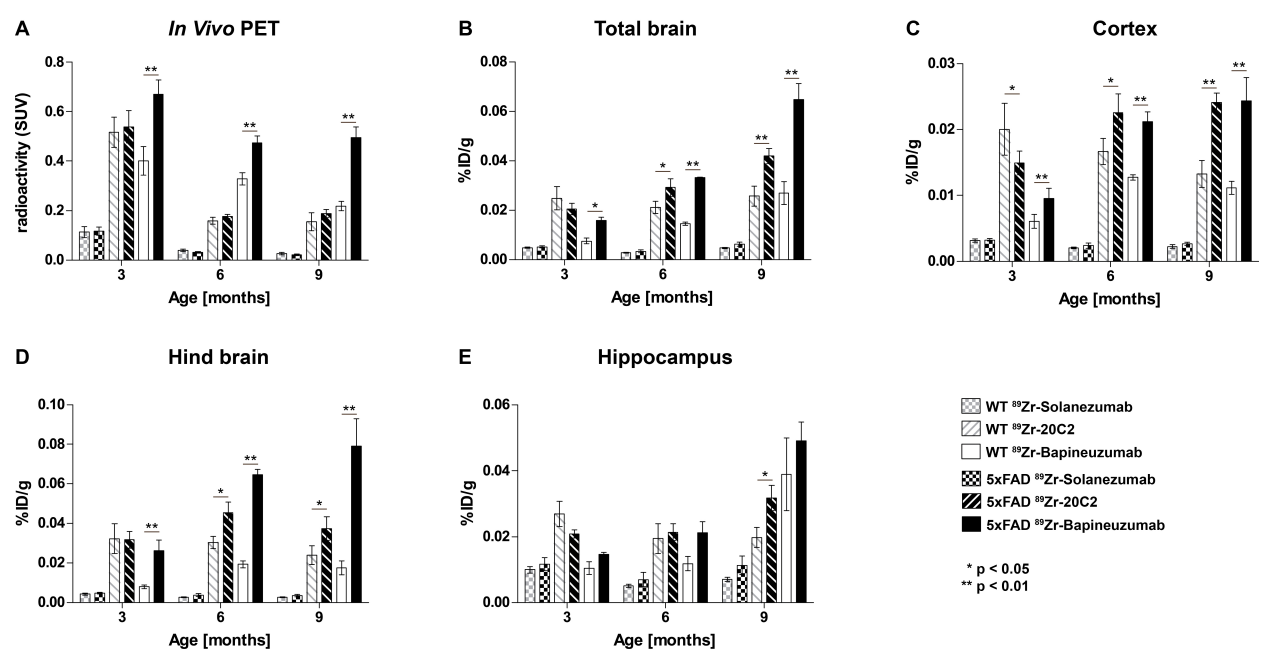

Figure 5.2: A) Animals were scanned five days after antibody administration. Increased uptake of ${ }^{89} \mathrm{Zr}$ bapineuzumab could be detected at all ages in 5xFAD animals. Both ${ }^{89} \mathrm{Zr}-20 \mathrm{C} 2$ and ${ }^{89} \mathrm{Zr}$-solanezumab did not show any increased accumulation in 5xFAD animals compared to WT animals. B) Biodistribution analysis of total brain showed an age-related accumulation of ${ }^{89} \mathrm{Zr}$-bapineuzumab at all ages in $5 x F A D$ animals. ${ }^{89} \mathrm{Zr}$ $20 \mathrm{C} 2$ also showed an age-related increase, but difference with WT animals only became apparent at the age of 6 months. ${ }^{89} \mathrm{Zr}$-solanezumab accumulated very little in the brain. C) ${ }^{89} \mathrm{Zr}$-bapineuzumab and ${ }^{89} \mathrm{Zr}$ solanezumab showed similar patterns in the cortex as in the total brain. However, at 3 months of age, ${ }^{89} \mathrm{Zr}$ 20C2 uptake was reduced in 5xFAD animals compared to WT animals. D) Hind brain results of all antibodies showed the same pattern as total brain accumulation, although the difference between WT and 5xFAD animals was bigger. E) In the hippocampus only ${ }^{89} \mathrm{Zr}-20 \mathrm{C} 2$ showed increased accumulation in $5 \mathrm{xFAD}$ animals, and this only at the late age of 9 months. ( $n=2-7$ per group, error bars denote SEM).

\subsubsection{BIODISTRIBUTION SHOWS AGE-DEPENDENT DIFFERENCE IN DISTRI- BUTION}

After in vivo $\mu$ PET scans, the brain was dissected and measured in a gamma-counter. These results confirmed that ${ }^{89} \mathrm{Zr}$-solanezumab does not accumulate in the brain, since radioactivity was on average five-fold lower compared to ${ }^{89} \mathrm{Zr}-20 \mathrm{C} 2$ and ${ }^{89} \mathrm{Zr}$ bapineuzumab in both WT and 5xFAD animals ( $<<0.01)$ (see Figure 5.2B).

${ }^{89} \mathrm{Zr}$-bapineuzumab uptake was increased in total brain in 5xFAD animals at the early age of 3 months $(p=0.03)$ and at later ages $(p<0.01)$. At 3 months of age, there was no difference in ${ }^{89} \mathrm{Zr}-20 \mathrm{C} 2$ uptake in WT and $5 \times \mathrm{xFA}$ animals $(\mathrm{p}=0.25)$. However, at 6 months of age, more ${ }^{89} \mathrm{Zr}-20 \mathrm{C} 2$ antibody was present in the total brain of $5 \mathrm{xFAD}$ $(p=0.04)$ and this increase was also present at 9 months of age $(p<0.01)$ (see Figure 5.2B).

${ }^{89} \mathrm{Zr}$-solanezumab did not show any specific accumulation at any age $(\mathrm{p}=0.73)$.

In the hind brain the uptake pattern was similar to that in total brain (see Figure 5.2D). ${ }^{89} \mathrm{Zr}$-bapineuzumab uptake was significantly higher at all ages in 5xFAD animals $(p<0.01)$, while for ${ }^{89} \mathrm{Zr}-20 \mathrm{C} 2$ only at 6 and 9 months of age an increased uptake in $5 \mathrm{xFAD}$ animals was present $(\mathrm{p}=0.02$ and $\mathrm{p}=0.04$ respectively). There was no difference in uptake of ${ }^{89} \mathrm{Zr}$-solanezumab in any of the animals or at the ages.

Unexpectedly, ${ }^{89} \mathrm{Zr}$-20C2 uptake in the cortex was lower at 3 months of age in $5 \mathrm{xFAD}$ animals compared to WT animals ( $\mathrm{p}=0.04$ ). At 6 and 9 months of age, the difference was 
reversed, with a higher ${ }^{89} \mathrm{Zr}$-20C2 uptake in 5xFAD animals than in WT animals $(\mathrm{p}=0.02$ and $\mathrm{p}<0.01$ respectively). ${ }^{89} \mathrm{Zr}$-solanezumab showed no difference in uptake at any age. ${ }^{89} \mathrm{Zr}$-bapineuzumab meanwhile, showed significant increased uptake at all ages $(\mathrm{p}<0.01)$ as reported previously.

In the hippocampus, only ${ }^{89} \mathrm{Zr}-20 \mathrm{C} 2$ showed a significant increased accumulation in $5 \mathrm{xFAD}$ animals, and this only at 9 months of age $(\mathrm{p}=0.01)$ (see Figure 5.2E). Both ${ }^{89} \mathrm{Zr}$-solanezumab and ${ }^{89} \mathrm{Zr}$-bapineuzumab showed no significant accumulation in the hippocampus of $5 x F A D$ animals at any of the ages, but there was trend for ${ }^{89} \mathrm{Zr}$ bapineuzumab accumulation in the hippocampus of 5xFAD animals at 6 and 9 months of age ( $\mathrm{p}=0.06$ in both cases).

A

Lymphnodes

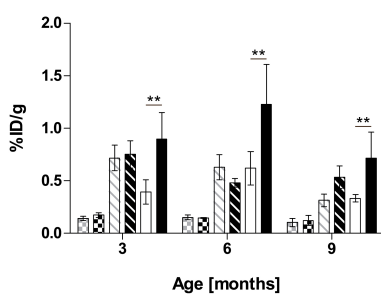

준 WT $^{89} \mathrm{Zr}$-Solanezumab

$\square \mathrm{WT}{ }^{\mathrm{B}} \mathrm{Zr}-20 \mathrm{C2}$

$\square$ WT ${ }^{89} \mathrm{Zr}$-Bapineuzumab
B

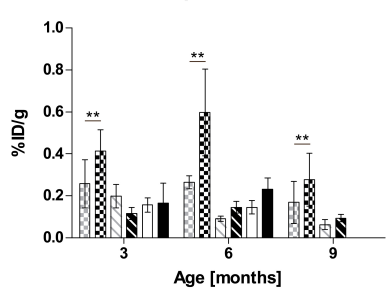

Q 5xFAD ${ }^{89} \mathrm{Zr}$-Solanezumab VI 5xFAD ${ }^{89} \mathrm{Zr}-20 \mathrm{C} 2$

5xFAD ${ }^{89} Z r-B a p i n e u z u m a b$

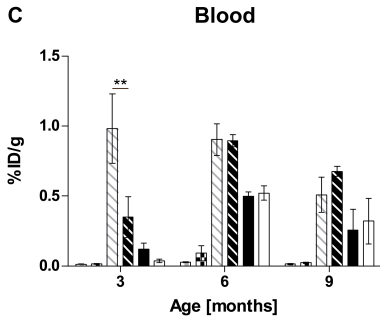

Figure 5.3: Biodistribution analysis of other organs revealed significant, specific antibody uptake in a number of organs. A) In cervical lymphnodes, increased ${ }^{89} \mathrm{Zr}$-bapineuzumab accumulation was found in $5 \mathrm{xFAD}$ animals at all ages. Other antibodies did not show this accumulation. B) In visceral adipose tissue, we found a significant increase in ${ }^{89} \mathrm{Zr}$-solanezumab in $5 x F A D$ animals. No increased accumulation of other antibodies was present. C) ${ }^{89} \mathrm{Zr}$-20C2 shows increased clearance from blood in 5xFAD animals at 3 months of age. At other ages, no difference in clearance was present between genotypes. Other antibodies also did not show any AD specific differences in clearance at any age. ( $n=2-7$ per group, error bars denote SEM).

\subsection{4. ${ }^{89}$ ZR-SOLANEZUMAB SHOWS INCREASED UPTAKE IN ADIPOSE TISSUE}

Biodistribution analysis also yielded important information about distributional differences between the three different antibodies. As previously reported, ${ }^{89} \mathrm{Zr}$ bapineuzumab showed increased uptake in cervical lymphnodes of 5xFAD animals ( $<<0.01$ ), while this accumulation was absent with ${ }^{89} \mathrm{Zr}$-solanezumab and ${ }^{89} \mathrm{Zr}$-20C2 (see Figure 5.3A).

Surprisingly, there was increased accumulation of ${ }^{89} \mathrm{Zr}$-solanezumab in abdominal adipose tissue in 5xFAD animals at all ages ( $\mathrm{p}=0.04) .{ }^{89} \mathrm{Zr}-20 \mathrm{C} 2$ and ${ }^{89} \mathrm{Zr}$-bapineuzumab did not show accumulation in this tissue, suggesting that only monomeric $A \beta$ accumulates in adipose tissue (see Figure 5.2B). In addition, there was a strong correlation ( $\mathrm{r}=0.70)$ between ${ }^{89} \mathrm{Zr}$-solanezumab uptake in adipose tissue and $\mathrm{A} \beta$ load in the brain $(\mathrm{p}=0.01)$.

Biodistribution analysis revealed that ${ }^{89} \mathrm{Zr}-20 \mathrm{C} 2$ was cleared from the blood faster in 3 months old 5xFAD animals compared to WT animals $(\mathrm{p}<0.01)$. At later ages, this difference in clearance between genotypes was not present. Furthermore, the other two antibodies did not show a significant difference in antibody clearance between 
genotypes (see Figure 5.3C). These results correlated ( $\mathrm{r}=0.66)$ with the results found in the cortex for ${ }^{89} \mathrm{Zr}-20 \mathrm{C} 2(\mathrm{p}<0.01)$, which showed lower cortical accumulation in 5xFAD mice at 3 months of age. In ${ }^{89} \mathrm{Zr}-20 \mathrm{C} 2$ treated $5 \mathrm{xFAD}$ animals, total activity was also lower at 3 months of age compared to WT animals, showing that the antibody actually gets eliminated from the body $(\mathrm{p}<0.01)$. At 9 months of age, this pattern was reversed, with increased total accumulation of ${ }^{89} \mathrm{Zr}-20 \mathrm{C} 2$ in $5 \mathrm{xFAD}$ animals $(\mathrm{p}=0.01$ ) (see Figure 5.4). 

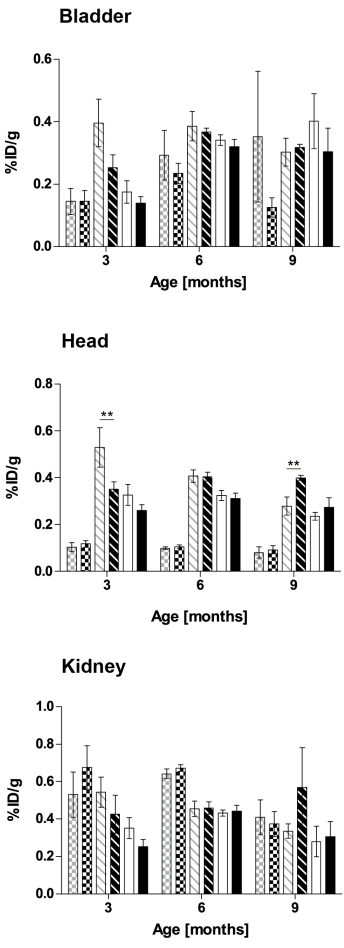

Lungs
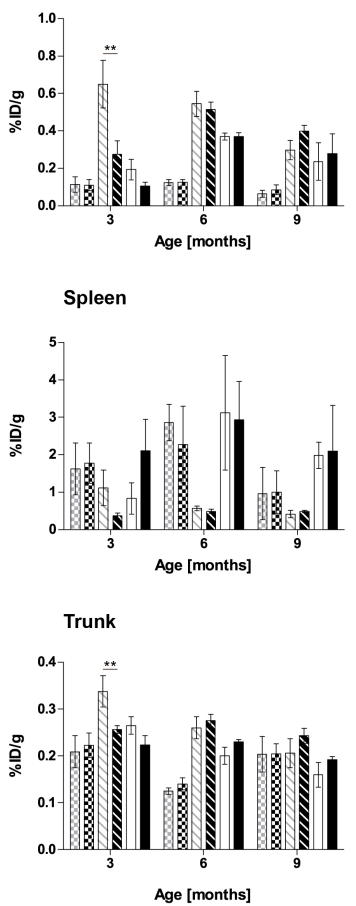
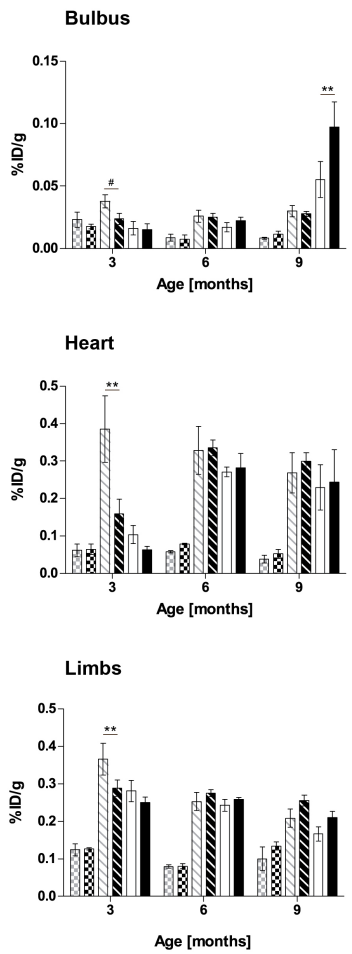

Salivary Glands

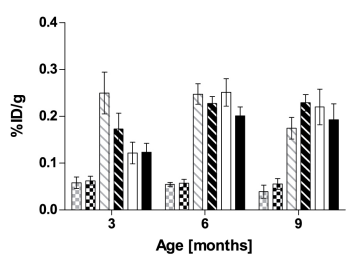

Stomach

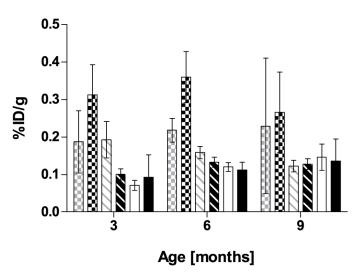

Total

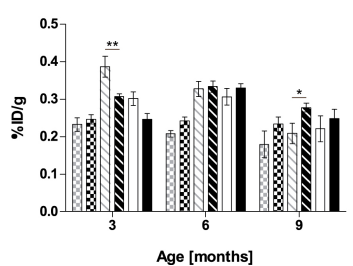

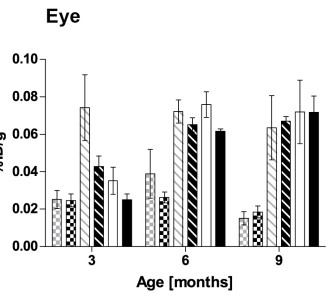
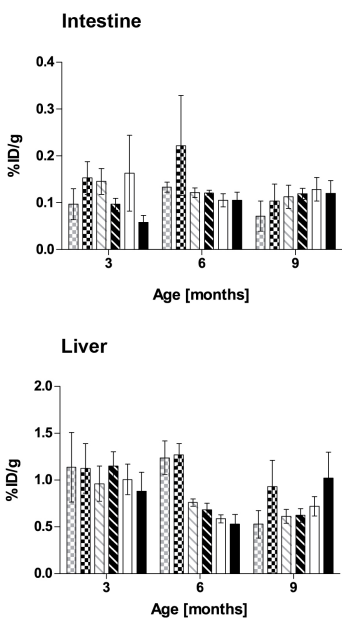

Skin

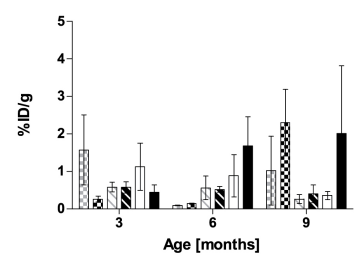

Tail
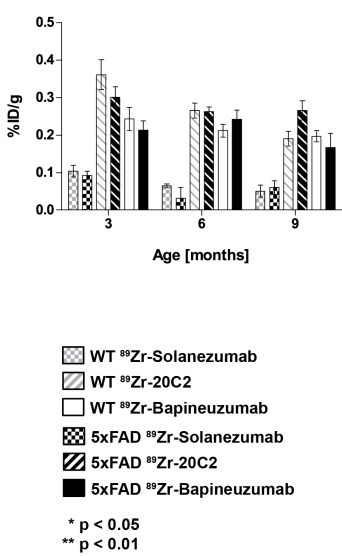

Figure 5.4: Overview of biodistribution data from all analyzed organs. The increased clearance of ${ }^{89} \mathrm{Zr}-20 \mathrm{C} 2$ in 3 months old 5xFAD animals also affected radioactivity levels in other organs such as the bulbus, head (without eyes and brain), heart, limbs, lungs, trunk and total body radioactivity. In addition, a significant increase of ${ }^{89} \mathrm{Zr}$-bapineuzumab was present in the bulbus of 9 months old $5 \mathrm{xFAD}$ animals. ( $\mathrm{n}=2-7$ per group, error bars denote SEM). 


\subsection{DISCUSSION}

This study compares the in vivo and ex vivo binding properties of three different antibodies targeting different $A \beta$ conformational forms (monomers, oligomers, fibrils) using immuno-PET. First, our ex vivo results showed that monomeric A $\beta$ load does not increase as animals age. In contrast, oligomers and fibrillary $A \beta$ (plaques), did show an age-dependent increase in both plaque load and in the amount of plaques of different size.

In vivo analysis using immuno-PET showed that only ${ }^{89} \mathrm{Zr}$-bapineuzumab accumulates in the brain at all ages. When measuring radioactivity in the different brain regions using biodistribution, which has a better signal-to-noise ratio, we found that also ${ }^{89} \mathrm{Zr}$ 20C2 (oligomers) showed an age-related accumulation in 5xFAD animals at 6 and 9 months of age.

Surprisingly, there was less ${ }^{89} \mathrm{Zr}-20 \mathrm{C} 2$ present in the cortex in 3 months old $5 x F A D$ animals compared to WT animal. Furthermore, there was also less ${ }^{89} \mathrm{Zr}-20 \mathrm{C} 2$ present in blood and in the total animal, showing that ${ }^{89} \mathrm{Zr}-20 \mathrm{C} 2$ was eliminated at a higher rate at this young age in $5 x F A D$ animals. A possible explanation for these results is that while oligomers are still soluble at a young age, ${ }^{89} \mathrm{Zr}-20 \mathrm{C} 2$ can actually remove them from a brain. At later ages, oligomers might get converted to insoluble forms and incorporated in $\mathrm{A} \beta$ plaques, which is also supported by the ex vivo plaque load analysis.

In accordance with the ex vivo $\mathrm{A} \beta$ quantification, we did not find any age-related increase of ${ }^{89} \mathrm{Zr}$-solanezumab in $5 \mathrm{xFAD}$ animals. However, there was also no increased binding compared to WT animals. The fact that ${ }^{89} \mathrm{Zr}$-solanezumab did not show any accumulation in the $5 x F A D$ brain at any age, confirms previous reports that solanezumab does not accumulate in the brain, but rather acts as a peripheral $A \beta$ sink [12].

Interestingly, there was specific uptake of $\left[{ }^{89} \mathrm{Zr}\right]$-solanezumab in visceral adipose tissue in 5xFAD mice. While it has been reported that solanezumab can also bind non-specifically to serum proteins [27], we did not find this increase in WT animals, showing that this accumulation was AD specific. In the $5 \times$ FAD animal model, APP is expressed under the Thyl promoter [13]. A possible explanation for this accumulation could be that this promoter is also active in adipose tissue. However, research on Thy1YFP reporter mice shows that the Thyl promoter is not active in adipose tissue [28]. In addition, we also found a strong correlation with monomeric plaque load in the brain. This accumulation warrants further investigation, since there is a strong link between metabolic syndrome and AD [29]. Furthermore, this insight might provide some clue as to possible therapeutic mechanisms of solanezumab.

These results also provide a hint regarding the working mechanisms of immunotherapy for AD. From the human clinical trials, we know that bapineuzumab did not show any beneficial effect [5], while solanezumab showed a small beneficial effect, but only in patients with mild $\mathrm{AD}$ [10]. This study proves that both antibodies work in completely different ways, with solanezumab not accumulating in the brain, while bapineuzumab does accumulate, and already at a young age. It would therefore be interesting to test ${ }^{89} \mathrm{Zr}$-20C2 in an intervention study and see whether it works in a prevention or therapeutic regimen, since the binding properties of this antibody change as animals age. 
One limitation of this study is that these antibodies were only compared in one animal model. While the 5xFAD animal model is extensively used, several other animal models are available which might provide different insights into plaque formation and age-related deposition [30].

In conclusion, we show the binding properties of different $A \beta$ antibodies in vivo. By making use of ${ }^{89} \mathrm{Zr}$-labeled antibodies, we were able to show that oligomers get removed by anti-amyloid administration only at a young age. In addition, we show accumulation of $\mathrm{A} \beta$ monomers in adipose tissue, a finding which warrants further investigation. 


\section{REFERENCES}

[1] Schneider LS, Mangialasche F, Andreasen N, Feldman H, Giacobini E, Jones R, et al. Clinical trials and late-stage drug development for Alzheimer's disease: an appraisal from 1984 to 2014 [Journal Article]. Journal of Internal Medicine. 2014;275(3):251283. Available from: http://dx.doi.org/10.1111/joim.12191.

[2] Miles LA, Crespi GAN, Doughty L, Parker MW. Bapineuzumab captures the Nterminus of the Alzheimer's disease amyloid-beta peptide in a helical conformation [Journal Article]. Scientific Reports. 2013;3:1302. Available from: http://dx . doi . org/10.1038/srep01302.

[3] Zago W, Buttini M, Comery TA, Nishioka C, Gardai SJ, Seubert P, et al. Neutralization of Soluble, Synaptotoxic Amyloid $\beta$ Species by Antibodies Is Epitope Specific [Journal Article]. The Journal of Neuroscience. 2012;32(8):2696-2702. Available from: http: //www. jneurosci .org/content/32/8/2696. abstract.

[4] Salloway S, Sperling R, Gilman S, Fox NC, Blennow K, Raskind M, et al. A phase 2 multiple ascending dose trial of bapineuzumab in mild to moderate Alzheimer disease [Journal Article]. Neurology. 2009;73(24):2061-2070. Available from: http: //www.ncbi.nlm.nih.gov/pmc/articles/PMC2790221/.

[5] Salloway S, Sperling R, Fox NC, Blennow K, Klunk W, Raskind M, et al. Two Phase 3 Trials of Bapineuzumab in Mild-to-Moderate Alzheimer's Disease [Journal Article]. New England Journal of Medicine. 2014;370(4):322-333. Available from: http:// www.nejm.org/doi/full/10.1056/NEJMoa1304839.

[6] Liu E, Schmidt ME, Margolin R, Sperling R, Koeppe R, Mason NS, et al. Amyloid$\beta 11 \mathrm{C}-\mathrm{PiB}-\mathrm{PET}$ imaging results from 2 randomized bapineuzumab phase $3 \mathrm{AD}$ trials [Journal Article]. Neurology. 2015;85(8):692-700.

[7] Blennow K, Hampel H, Zetterberg H. Biomarkers in Amyloid-ßImmunotherapy Trials in Alzheimer/'s Disease [Journal Article]. Neuropsychopharmacology. 2014;39(1):189-201. Available from: http://dx.doi.org/10.1038/npp. 2013. 154.

[8] Carrillo MC, Brashear HR, Logovinsky V, Ryan JM, Feldman HH, Siemers ER, et al. Can we prevent Alzheimer's disease? Secondary "prevention" trials in Alzheimer's disease [Journal Article]. Alzheimer's \& Dementia. 2013;9(2):123131.e1. Available from: http://www.sciencedirect.com/science/article/ pii/S1552526012025873.

[9] Crespi GAN, Hermans SJ, Parker MW, Miles LA. Molecular basis for midregion amyloid- $\beta$ capture by leading Alzheimer's disease immunotherapies [Journal Article]. Scientific Reports. 2015;5:9649. Available from: http://dx . doi .org/10 . 1038/srep09649.

[10] Doody RS, Thomas RG, Farlow M, Iwatsubo T, Vellas B, Joffe S, et al. Phase 3 Trials of Solanezumab for Mild-to-Moderate Alzheimer's Disease [Journal Article]. New 
England Journal of Medicine. 2014;370(4):311-321. Available from: http://www . nejm.org/doi/full/10.1056/NEJMoa1312889.

[11] Farlow M, Arnold SE, van Dyck CH, Aisen PS, Snider BJ, Porsteinsson AP, et al. Safety and biomarker effects of solanezumab in patients with Alzheimer's disease [Journal Article]. Alzheimer's \& Dementia. 2012;8(4):261-271. Available from: http : / www . sciencedirect.com/science/article/pii/S1552526011029438.

[12] DeMattos RB, Bales KR, Cummins DJ, Dodart JC, Paul SM, Holtzman DM. Peripheral anti-Aßantibody alters CNS and plasma A $\beta$ clearance and decreases brain Aßburden in a mouse model of Alzheimer's disease [Journal Article]. Proceedings of the National Academy of Sciences of the United States of America. 2001;98(15):8850-8855. Available from: http://www.ncbi.nlm.nih.gov/pmc/ articles/PMC37524/.

[13] Oakley H, Cole SL, Logan S, Maus E, Shao P, Craft J, et al. Intraneuronal $\beta$ Amyloid Aggregates, Neurodegeneration, and Neuron Loss in Transgenic Mice with Five Familial Alzheimer's Disease Mutations: Potential Factors in Amyloid Plaque Formation [Journal Article]. The Journal of Neuroscience. 2006;26(40):1012910140. Available from: http://www.jneurosci.org/content/26/40/10129. abstract.

[14] Stevens J, Martinez-Martinez P, Dubois L, Vugts D, Nell T, Crivelli S, et al. Brain uptake of a therapeutic amyloid antibody and in vivo PET imaging in an Alzheimer's disease model; 2016.

[15] Hardy JA, Higgins GA. Alzheimer's disease: the amyloid cascade hypothesis [Journal Article]. Science. 1992;256(5054):184.

[16] Walsh DM, Selkoe DJ. AßOligomers - a decade of discovery [Journal Article]. Journal of Neurochemistry. 2007;101(5):1172-1184. Available from: http://dx.doi.org/ $10.1111 / j .1471-4159.2006 .04426 . x$.

[17] Lambert MP, Viola KL, Chromy BA, Chang L, Morgan TE, Yu J, et al. Vaccination with soluble Aßoligomers generates toxicity-neutralizing antibodies [Journal Article]. Journal of Neurochemistry. 2001;79(3):595-605. Available from: http://dx.doi . org/10.1046/j.1471-4159.2001.00592.x.

[18] Haes AJ, Chang L, Klein WL, Van Duyne RP. Detection of a Biomarker for Alzheimer's Disease from Synthetic and Clinical Samples Using a Nanoscale Optical Biosensor [Journal Article]. Journal of the American Chemical Society. 2005;127(7):2264-2271. Available from: http://dx.doi.org/10.1021/ja044087q.

[19] Black R, Ekman L, Lieberburg I, Grundman M, Callaway J, Gregg KM, et al.. Immunotherapy Regimes Dependent On APOE Status. Google Patents; 2008.

[20] Holtzman DM, DeMattos R, Bales KR, Paul SM, Tsurushita N, Vasquez M. Humanized antibodies that sequester amyloid beta peptide. Google Patents; 2011. 
[21] Kinney G, Strohl WR, An Z. Anti-ADDL monoclonal antibody and use thereof. Google Patents; 2010.

[22] Alakurtti K, Johansson JJ, Joutsa J, Laine M, Backman L, Nyberg L, et al. Long-term test-retest reliability of striatal and extrastriatal dopamine D2/3 receptor binding: study with [lsqb]11C[rsqb]raclopride and high-resolution PET [Journal Article]. J Cereb Blood Flow Metab. 2015;Available from: http://dx.doi.org/10.1038/ jcbfm.2015.53.

[23] Granton PV, Dubois L, van Elmpt W, van Hoof SJ, Lieuwes NG, De Ruysscher D, et al. A Longitudinal Evaluation of Partial Lung Irradiation in Mice by Using a Dedicated Image-Guided Small Animal Irradiator [Journal Article]. International Journal of Radiation Oncology*Biology*Physics. 2014;90(3):696-704. Available from: http: //www.sciencedirect.com/science/article/pii/S0360301614034713.

[24] Abou DS, Ku T, Smith-Jones PM. In vivo biodistribution and accumulation of 89Zr in mice [Journal Article]. Nuclear Medicine and Biology. 2011;38(5):675681. Available from: http://www.sciencedirect.com/science/article/pii/ S0969805110005214.

[25] Edelstein AD, Tsuchida MA, Amodaj N, Pinkard H, Vale RD, Stuurman N. Advanced methods of microscope control using $\mu$ Manager software [Journal Article]. Journal of Biological Methods. 2014;1(2):e10.

[26] Preibisch S, Saalfeld S, Tomancak P. Globally optimal stitching of tiled 3D microscopic image acquisitions [Journal Article]. Bioinformatics. 2009;25(11):1463-5. Available from: http://www.ncbi.nlm.nih.gov/pubmed/19346324.

[27] Watt A, Crespi GN, Down R, Ascher D, Gunn A, Perez K, et al. Do current therapeutic anti-Aßantibodies for Alzheimer's disease engage the target? [Journal Article]. Acta Neuropathologica. 2014;127(6):803-810. Available from: http://dx.doi.org/ $10.1007 / \mathrm{s} 00401-014-1290-2$.

[28] Jósvay K, Winter Z, Katona RL, Pecze L, Marton A, Buhala A, et al. Besides neuroimaging, the Thyl-YFP mouse could serve for visualizing experimental tumours, inflammation and wound-healing [Journal Article]. Scientific Reports. 2014;4:6776. Available from: http://dx.doi.org/10.1038/srep06776.

[29] De Felice FG. Alzheimer's disease and insulin resistance: translating basic science into clinical applications [Journal Article]. The Journal of Clinical Investigation. 2013;123(2):531-539. Available from: http://www.jci.org/articles/view/ 64595.

[30] Bouter Y, Noguerola JSL, Tucholla P, Crespi GAN, Parker MW, Wiltfang J, et al. Abeta targets of the biosimilar antibodies of Bapineuzumab, Crenezumab, Solanezumab in comparison to an antibody against $\mathrm{N}$-truncated Abeta in sporadic Alzheimer disease cases and mouse models [Journal Article]. Acta Neuropathologica. 2015;130(5):713-729. Available from: http://dx.doi.org/ $10.1007 / \mathrm{s} 00401-015-1489-\mathrm{x}$. 

DISCUSSION 
This thesis investigates the potential use of amyloid- $\beta$ (A $\beta$ ) antibodies for both the treatment and possible diagnosis of Alzheimer's disease (AD). While enormous research efforts are directed towards a cure for $\mathrm{AD}$, no disease modifying treatment has been achieved in the last 15 years. The most important hallmarks of $\mathrm{AD}$ are $\mathrm{A} \beta$ plaques and tau tangles [1]. We investigated the role of anti-A $\beta$ antibodies in $\mathrm{AD}$ as possible therapies, to learn more about antibody effector mechanisms and their potential for in vivo diagnostics in the CNS.

\subsection{SUMMARY OF KEY FINDINGS}

Chapter 2 presents an up-to-date literature review about the role of neuroinflammation in $\mathrm{AD}$ and the current state of the art of anti-A $\beta$ immunotherapy. In the last years, neuroinflammation has emerged as one of the key players of AD pathology [2]. While neuroinflammation is a complex interplay of several factors, the two most important factors in $\mathrm{AD}$ are microglia and complement. Besides the microglial changes observed in patients, the microglial association with $\mathrm{AD}$ has become prominent with the emergence of CD33 and TREM2 as risk factors for $\mathrm{AD}$ [3]. In addition to microglia, the complement system plays an important role in $\mathrm{AD}$ [4]. Genetic evidence points to Complement Receptor 1 (CR1) playing a crucial role in AD development, while evidence from patients and mouse models points to a further important role of complement factors and the inflammasome in $\mathrm{AD}$ pathogenesis.

Chapter 2 also further elaborates on the past and current immunotherapy efforts targeting $A \beta$ as a possible treatment for AD. From animal studies, several possible effector mechanisms have been proposed and investigated, although our literature search showed that there is a lack of attention to the role that complement might play in immunotherapy. In human clinical trials, we find that already 25 different immunotherapies have been or are being tried in over 25000 patients. So far most therapies have ended with negative results, although some results suggest that immunotherapy might hold a benefit in the early disease stages [5].

In Chapter 3, we investigated the effector mechanisms of $A \beta$ immunotherapy in a therapeutic and preventive fashion in the $5 \mathrm{xFAD}$ mouse model. To this end, an anti-inflammatory (a modified mouse IgG1) and an inflammatory (mouse IgG2a) anti-A $\beta$ antibody (3D6) were administered to 5xFAD mice before (preventive) and after (therapeutic) the onset of behavioral symptoms and overt plaque pathology. Surprisingly, we found that antibody administration resulted in IgG mediated passive systemic anaphylaxis (PSA) caused by generation of anti-therapeutic antibodies (ATA). Preventive treatment was successful in reducing anxiety-like effects in 5xFAD animals. Our results also showed that mIgG 3D6 worsens memory problems in the contextual fear-conditioning test.

On a molecular level, we discovered that preventive treatment resulted in a stronger reduction on plaque load than therapeutic treatment. We also investigated the effects of immunotherapy on the different $A \beta$ fractions in brain homogenates (water soluble, detergent soluble and formic acid soluble $A \beta$ ). While therapeutic treatment lowered $A \beta$ in the detergent soluble fraction, preventive treatment on the other hand increased $A \beta$ in the detergent soluble fraction, caused by a shift from the formic acid soluble fraction to the detergent soluble fraction. No effects on microgliosis were observed, but our 
data shows that treatment with inflammatory antibodies (mIgG2a) significantly reduced astrogliosis. These results suggest an important role for astrocytes in the mediation of neuroinflammatory processes.

Given the fact that mIgG1 3D6 (anti-inflammatory) induced an isotype specific worsening of memory, while mIgG2a 3D6 (inflammatory) reduced astrogliosis, it is likely that isotype specific effects on inflammation need to be taken into account when designing immunotherapies for AD. Furthermore, our results suggest that a more proinflammatory therapy might be more beneficial for $\mathrm{AD}$ treatment.

Chapter 4 shows a proof-of-concept for the use of $A \beta$ antibodies for in vivo imaging of amyloid. In this experiment, an $A \beta$ specific antibody (bapineuzumab) was radiolabeled with a long-lived positron emitter $\left({ }^{89} \mathrm{Zr}\right)$ and compared with an isotype control (mAb 637) in the 5xFAD mouse model at several disease stages. This experiment showed that specific in vivo accumulation of antibodies can be detected using PET imaging. In addition, biodistribution experiments proved an age-related increase of ${ }^{89} \mathrm{Zr}$-bapineuzumab uptake in the brain. To further prove specificity, we could show that competition with unlabeled bapineuzumab reduced ${ }^{89} \mathrm{Zr}$-bapineuzumab uptake to background levels. No uptake of the isotype control could be found. Specific accumulation of administered antibody was further proved by autoradiography and by colocalisation of administered ${ }^{89} \mathrm{Zr}$-bapineuzumab with plaques in the brain.

The previous experiment formed the basis for a follow-up study comparing different $A \beta$ antibodies in Chapter 5. Using again 5xFAD mice of different ages, we now compared the in vivo binding properties of three $\mathrm{A} \beta$ antibodies targeting monomers (solanezumab), oligomers (20C2), and plaques (bapineuzumab). To our surprise, only ${ }^{89} \mathrm{Zr}$-bapineuzumab allows for in vivo detection of plaques. With ${ }^{89} \mathrm{Zr}$-solanezumab and ${ }^{89} \mathrm{Zr}-20 \mathrm{C} 2$ no increased in vivo binding could be found.

Biodistribution showed, as previously published, that ${ }^{89} \mathrm{Zr}$-solanezumab does not seem to enter or remain in the brain in large concentrations. However, at 6 months of age, there was a significant accumulation of ${ }^{89} \mathrm{Zr}-20 \mathrm{C} 2$ in $5 \mathrm{xFAD}$ animals, which binds oligomers, in the brain. More interestingly, we found that at 3 months of age, there is a reduction of ${ }^{89} \mathrm{Zr}-20 \mathrm{C} 2$ binding in the cortex of $5 \mathrm{xFAD}$ animals compared to WT animals, a situation that was reversed at the later ages of 6 and 9 months. Analysis of antibody concentration in the blood also shows that there ${ }^{89} \mathrm{Zr}-20 \mathrm{C} 2$ is cleared more rapidly at 3 months of age in $5 \mathrm{xFAD}$ animals than in other conditions, a situation we did not observe with the other antibodies.

While ${ }^{89} \mathrm{Zr}$-solanezumab was cleared very rapidly from the animals (resulting in low concentrations in both 5xFAD and WT animals), we did find a specific accumulation of ${ }^{89} \mathrm{Zr}$-solanezumab in adipose tissue. Since only ${ }^{89} \mathrm{Zr}$-solanezumab binding was increased in adipose tissue in $5 x F A D$ animals, it is likely that monomeric, soluble $A \beta$ accumulates in fat deposits in the body.

Finally, we also showed that ${ }^{89} \mathrm{Zr}$-bapineuzumab accumulated in cervical lymphnodes of 5xFAD animals. This might be due to draining amyloid present in these lymphnodes, although it is more likely that there is an antibody specific mechanism, since the other antibodies fail to show any accumulation in the lymphnodes. 


\subsection{LIMITATIONS}

While this thesis delivers significant contributions to the understanding of AD, and more specifically to the use of $A \beta$ antibodies in treatment and diagnosis, there are also some limitations to our studies. One significant limitation that all our studies share is the use of the same AD mouse model. Because $\mathrm{AD}$ is such a complex disease, animal models are widely used to encompass as much as possible of the different disease processes when investigating drugs. While spontaneously models such as certain species of dogs, cats, or nonhuman primates exist, the vast majority of the current disease models are either pharmacologically induced or based on transgenic rodents [6].

With regards to the mouse model used in our study (5xFAD), some remarks about possible translatability have to be made. First, in contrast to the majority of AD patients, $5 \mathrm{xFAD}$ mice start to develop AD pathology at a very young age (2-3 months) with behavioral deficits showing at about 5-6 months of age, which would translate to a human in its early thirties [7]. At this young age, results about the immune system in $\mathrm{AD}$ have to be carefully interpreted, since the immune system at old age is less active than at young age, because of a process called immuno-senescence [8].

In addition, as most transgenic $\mathrm{AD}$ mouse models, the $5 \mathrm{xFAD}$ model overexpresses human APP and PSEN1. A comparative study showed that they express about 6 -fold more APP than humans, while the numerous mutations in APP also cause an increased $A \beta 42 / A \beta 40$ ratio [9]. This increased $A \beta$ in the brain of $5 x F A D$ mice compared to humans might limit the translation of our immuno-PET imaging results, since less $A \beta$ will be available for the antibodies to bind to in humans (Chapter 4 and Chapter 5). On the other hand, PET imaging in humans is more sensitive than in mice and likely to result in a better signal-to-noise ratio, potentially offsetting the disadvantage lower amyloid load in the human brain [10]. Furthermore, the fact that we can also detect in vivo differences at a very early age in the $5 \mathrm{xFAD}$ mice, suggest that it should be possible to translate this technique to clinic.

Another limitation of Chapter 4 and Chapter 5 is the fact that no comparison could be made to existing amyloid PET tracers. Several amyloid PET tracers are currently being used in patients [11]. To get a good comparison about the sensitivity of immuno-PET imaging with existing amyloid PET tracers, one or more of these tracers would have preferentially been used in our animal model to enable a direct comparison.

Another limitation related to the use of the 5xFAD animal model relates to the unstable behavioral phenotype. While initial pilot studies showed a very strong phenotype (unpublished data), this could not be replicated in subsequent studies in immunotherapy (Chapter 3), resulting in a failure to find a difference between WT and untreated 5xFAD mice. A possible explanation for this might be the difference in breeding of our cohort compared to the original description of the 5xFAD model, were offspring is generated by breeding 5xFAD mice with B6/SJL F1 mice [7]. We on the other hand bred 5xFAD mice with B6/SJL F3 mice. In addition, the Pde6b gene, which can cause retinal degeneration, was bred out of our colony.

While investigating the effects of $A \beta$ immunotherapy using recombinant produced antibodies (Chapter 3), 5xFAD animals developed anti-treatment antibodies (ATA), resulting in IgG mediated passive anaphylactic shock. The development of ATA could have affected our results, because therapeutic antibodies could be neutralized and not 
reach the brain. In addition, systemic inflammatory reaction caused by the anaphylactic shock, could also have influenced the inflammatory processes in the brain.

\subsection{PeRspectives}

This thesis provides important insights into the use of antibodies for the treatment and diagnosis of $\mathrm{AD}$. Contrary to our expectations, results show that a more proinflammatory antibody might be more beneficial than an anti-inflammatory antibody, despite a stronger reduction in plaque load. The antibodies solanezumab and crenezumab both bind the same $A \beta$ epitope, while solanezumab has an inflammatory isotype (human IgG1) and crenezumab has an anti-inflammatory isotype (human IgG4). At the moment, solanezumab has completed phase 3 clinical trials with overall negative results, but possible showing a small effect in patients with mild $\mathrm{AD}$ [12]. Interim reports of a phase 2 trial of crenezumab have likewise delivered disappointing reports [13]. One difference with our pre-clinical experiment is that these antibodies target soluble, monomeric $A \beta$.

Finally, our immuno-PET studies offer an interesting tool to investigate $A \beta$ antibody distribution in vivo. PET imaging using ${ }^{89} \mathrm{Zr}$-labeled antibodies are routinely used in humans and several $\mathrm{A} \beta$ antibodies have already been tested in clinical trials in humans. It should therefore be only a relatively small step to conduct human trials using ${ }^{89} \mathrm{Zr}$ labeled $A \beta$ antibodies. This might yield important new information about disease progression and immunotherapy for $\mathrm{AD}$. In addition, this technique could maybe also be used to image other proteinopathies such as Parkinson's disease. 


\section{REFERENCES}

[1] Braak H, Braak E. Neuropathological stageing of Alzheimer-related changes [Journal Article]. Acta neuropathologica. 1991;82(4):239-259.

[2] Heneka MT, Carson MJ, Khoury JE, Landreth GE, Brosseron F, Feinstein DL, et al. Neuroinflammation in Alzheimer's disease [Journal Article]. The Lancet Neurology. 2015;14(4):388-405. Available from: http://www.sciencedirect . $\mathrm{com} / \mathrm{science/article/pii/S1474442215700165.}$

[3] Heppner FL, Ransohoff RM, Becher B. Immune attack: the role of inflammation in Alzheimer disease [Journal Article]. Nat Rev Neurosci. 2015;16(6):358-372. Available from: http://dx.doi.org/10.1038/nrn3880.

[4] Heneka MT, Golenbock DT, Latz E. Innate immunity in Alzheimer's disease [Journal Article]. Nat Immunol. 2015;16(3):229-236. Available from: http://dx.doi.org/ 10.1038/ni.3102.

[5] Siemers ER, Sundell KL, Carlson C, Case M, Sethuraman G, Liu-Seifert H, et al. Phase 3 solanezumab trials: Secondary outcomes in mild Alzheimer's disease patients [Journal Article]. Alzheimer's \& Dementia. 2015;.

[6] Van Dam D, De Deyn PP. Animal models in the drug discovery pipeline for Alzheimer's disease [Journal Article]. British journal of pharmacology. 2011;164(4):1285-1300.

[7] Oakley H, Cole S, Logan S, Maus E, Shao P, Craft J, et al. Intraneuronal betaamyloid aggregates, neurodegeneration, and neuron loss in transgenic mice with five familial Alzheimer's disease mutations: potential factors in amyloid plaque formation [Journal Article]. Journal of Neuroscience. 2006;26(40):10129.

[8] Gruver A, Hudson L, Sempowski G. Immunosenescence of ageing [Journal Article]. The Journal of pathology. 2007;211(2):144-156.

[9] Maarouf CL, Kokjohn TA, Whiteside CM, Macias MP, Kalback WM, Sabbagh MN, et al. Molecular Differences and Similarities Between Alzheimer's Disease and the 5XFAD Transgenic Mouse Model of Amyloidosis [Journal Article]. Biochemistry insights. 2013;6:1.

[10] Yao R, Lecomte R, Crawford ES. Small-Animal PET: What Is It, and Why Do We Need It? [Journal Article]. Journal of Nuclear Medicine Technology. 2012;40(3):157165. Available from: http://tech.snmjournals.org/content/40/3/157. abstract.

[11] Eshel N, Lieberman G. Imaging of Alzheimer's Disease: State of the Art [Journal Article]; 2015.

[12] Doody RS, Thomas RG, Farlow M, Iwatsubo T, Vellas B, Joffe S, et al. Phase 3 trials of solanezumab for mild-to-moderate Alzheimer's disease [Journal Article]. New England Journal of Medicine. 2014;370(4):311-321. 
[13] Cummings J, Cho W, Ward M, Friesenhahn M, Brunstein F, Honigberg L, et al. A RANDOMIZED, DOUBLE-BLIND, PLACEBO-CONTROLLED PHASE 2 STUDY TO EVALUATE THE EFFICACY AND SAFETY OF CRENEZUMAB IN PATIENTS WITH MILD TO MODERATE ALZHEIMER'S DISEASE [Journal Article]. Alzheimer's \& Dementia: The Journal of the Alzheimer's Association. 2014;10(4):P275. Available from: http://dx.doi.org/10.1016/j.jalz.2014.04.450. 



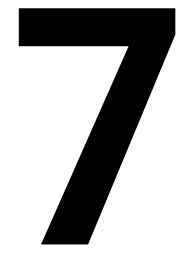

VALORISATION 
Alzheimer's disease (AD) is the leading cause of dementia worldwide. Over 46 million people suffer from dementia, with $\mathrm{AD}$ accounting for $60-80 \%$ of the cases and this number is expected to double every twenty years [1]. This means that about every 3 seconds, a new case of dementia will be diagnosed worldwide. By 2050, over 130 million people are expected to suffer from dementia, of which around 90 million will have AD.

The societal burden of AD is especially high, since not only the patient who has the dementia is affected, but also family and friends, and society as a whole by providing care and loss of productivity. In general, dementia patients require more intensive care than patients from other diseases, and the costs for dementia are higher than the combined costs for cancer, heart disease and stroke [2]. In addition, cognitive impairment, a symptom of dementia, is the strongest prediction for transition of elderly people to a care home [3].

In terms of economic value, the global cost of dementia exceeds 800 billion US\$, which accounts for over $1 \%$ of global gross domestic product (GDP). A minority of these costs $(20 \%)$ is attributable to direct medical care costs, possible because very few treatment options are available [1]. Direct social care (home, nursing) and informal care costs each attribute $40 \%$ to the costs. Informal care costs are costs made by unpaid informal care givers (such as relatives) who are for example unable to work during this time.

Despite the fact that the societal cost of $\mathrm{AD}$ is substantial higher than those of stroke, heart disease and cancer, research funding into $\mathrm{AD}$ is less than in these disease [4]. It is therefore imperative that both fundamental and applied research funding is increased to prevent an economical disaster in the coming decades. The problem is further aggravated by the fact that there is no cure for the disease available. In addition, the exact disease process remains vague and also diagnosis of $\mathrm{AD}$ is not straightforward [5].

The thesis contains both a therapeutic and a diagnostic component that offers direct possibilities for valorization. First we will discuss possible valorization options from a therapeutic point of view, while subsequently we will discuss diagnostic options suggested in this thesis.

\subsection{THERAPEUTIC OPPORTUNITIES}

At the moment, 5 different drugs are approved for AD. Every year, about 1 billion dollars are spent on these drugs in the USA alone [6]. While these drugs provide some symptomatic relief for patients, they do not tackle the underlying cause of AD, and as such, they lose their effectiveness over time.

In this thesis, immunotherapy against amyloid was investigated as a possible treatment option. Anti-A $\beta$ treatment has been tested in over 25000 patients in various clinical trials (Chapter 2). Although cost estimates are not available, these trials are likely to represent a huge investment on the part of pharmaceutical companies. Furthermore, there is a large societal costs and effort required both on the patient and caregiver side. Knowledge obtained in this thesis helps to improve future immunotherapy trials and possible increase the success rate of anti-A $\beta$ strategies, if these strategies are further pursued in the future.

Despite the fact that immunotherapy trials have failed so far, we show that preventive treatment might be a viable option for the treatment of $\mathrm{AD}$. One key question remains 
of course whether it will be possible to identify patients early enough. A big problem is that amyloid pathology starts about 15-20 years before the onset of cognitive problems [7]. It remains thus to be seen how it would be possible to select patients for possible treatment, or if treatment starting in the state of Mild Cognitive Impairment (MCI) is sufficient to obtain a disease modifying effect.

Our results show that there might be valorization opportunities in custom antibody formats and effector mechanisms. In this thesis, an antibody with full effector mechanisms ( $\mathrm{Fc} \gamma$-receptor binding and complement activation) was compared with an antibody with no effector mechanisms. A possible valorization opportunity would be to investigate antibodies with partial effector mechanisms (eg. only complement activation or only $\mathrm{Fc} \gamma$-receptor binding) and see which mechanisms is the most important for an efficient and safe therapy. This specific modification could then be patented. Additionally, it might also be worthwhile to investigate different, more exotic antibody formats, such as bispecific antibodies to increase brain penetration and increase the effective dose in the brain.

\subsection{DIAGNOSTIC OPPORTUNITIES}

While $\mathrm{AD}$ can only be diagnosed with certainty post-mortem, a number of options are currently available for early diagnosis of the disease. As discussed in Chapter 1 of these thesis, diagnostic tools currently in common use are cognitive tests and CSF measurements. $A \beta$ and tau measurements in CSF are now routinely being used as part of the neurological examination in patients with cognitive impairments. However, besides these measures, also imaging biomarkers are being routinely used. One advantage over imaging techniques is that they also provide additional spatial information as to where pathology is spreading and about the severity of the pathology. While structural Magnetic Resonance Imaging (MRI) is routinely used for diagnosis, the use of Positron Emission Tomography (PET) to image amyloid is now finding routine use.

One of the disadvantages of current amyloid PET tracers is that they are only able to image fibrillar $A \beta$ deposits (plaques). However, recent experimental data suggests that it is actually the oligomeric $A \beta$ species which are more toxic. It would therefore be of great diagnostic and scientific value if different $A \beta$ species could be imaged. This thesis proves in pre-clinical studies that this might be feasible.

The use of monoclonal antibodies radiolabeled with ${ }^{89}$ Zirconium is routinely applied in the clinic for imaging of tumors [8]. Despite the fact that radiation dose is always a concern with PET imaging, the technology has proven safe in humans. We, and very recently also others, have shown that antibodies can be used for imaging amyloid in the brain in vivo $[9,10]$. This opens opportunities to try this technology in humans. Since both the use of ${ }^{89} \mathrm{Zr}$ and some of the antibodies we tested have already passed clinical trials, and proven safe, amyloid immuno-PET could be tried very fast in humans. This could provide valuable new insights in amyloid pathology in patients. In vivo imaging using this technique in mice suffers from several drawbacks, such as the high bone uptake of ${ }^{89} \mathrm{Zr}$ [11]. In humans, this problem would not occur, and the technique would therefore benefit from a higher signal-to-noise ratio.

Besides applications for diagnosis and fundamental science, immuno-PET also offers the possibility to use monoclonal antibodies as a theranostic tool. Since several 
antibodies are still in clinical trial, this would provide a valuable addition to current clinical trials. A theranostic is a drug that is a diagnostic tool and a therapeutic drug at the same time. By using the same antibody that is used for therapy for imaging pathology, target engagement can be shown on an individual patient basis. This will allow to screen patients before inclusion in clinical trials, possibly increasing success of the trial and preventing people with non-AD dementia from being included in the trial. Furthermore, therapy effects could be monitored over time.

Finally, the fact that immuno-PET can be used for $A \beta$ imaging, as we show in this thesis, might also mean that immuno-PET can be used to target other proteins in the CNS. Applications for immuno-PET might therefore include also other proteinopathies such as Parkinson's disease, for which no tracers are available for the moment. Monoclonal antibodies against $\alpha$-synuclein could be radiolabeled and used to confirm Parkinson's disease. Other possible disease could be for instance Prion disease, but also in $\mathrm{AD}$ there are still possible targets left. Besides $\mathrm{A} \beta$, also tau might prove a valuable target to image with immuno-PET, and currently there are only few tau tracers available, and these are still in early development. A possible caveat is that tau is intracellular, and it remains an open question whether antibodies will be able to bind then. However, results from a study using tau antibodies for in vivo imaging system (IVIS) imaging, shows that this might not be such a big issue [12]. 


\section{REFERENCES}

[1] Prince M, Wimo A, Guerchet M, Ali G, Wu Y, Prina M. World Alzheimer Report 2015. The global impact of dementia. An analysis of prevalence, incidence, cost and trends [Journal Article]. Alzheimer's Disease International, London. 2015;

[2] Luengo-Fernandez R, Leal J, Gray A. Dementia 2010: The prevalence, economic cost and research funding of dementia compared with other major diseases [Journal Article]. Cambridge: Alzheimer's Research Trust. 2010;.

[3] Gaugler JE, Duval S, Anderson KA, Kane RL. Predicting nursing home admission in the US: a meta-analysis [Journal Article]. BMC geriatrics. 2007;7(1):1.

[4] Lowin A, Knapp M, McCrone P. Alzheimer's disease in the UK: comparative evidence on cost of illness and volume of health services research funding [Journal Article]. International journal of geriatric psychiatry. 2001;16(12):1143-1148.

[5] Karantzoulis S, Galvin JE. Distinguishing Alzheimer's disease from other major forms of dementia [Journal Article]. Expert review of neurotherapeutics. 2014;

[6] Casey DA, Antimisiaris D, O’Brien J. Drugs for Alzheimer's disease: are they effective? [Journal Article]. Pharmacy and Therapeutics. 2010;35(4):208.

[7] Jack CR, Knopman DS, Jagust WJ, Petersen RC, Weiner MW, Aisen PS, et al. Update on hypothetical model of Alzheimer's disease biomarkers [Journal Article]. Lancet neurology. 2013;12(2):207-216. Available from: http: //www.ncbi.nlm.nih.gov/ pmc/articles/PMC3622225/.

[8] Vugts DJ, van Dongen GAMS. 89Zr-labeled compounds for PET imaging guided personalized therapy [Journal Article]. Drug Discovery Today: Technologies. 2011;8(2-4):e53-e61. Available from: http://www.sciencedirect.com/ science/article/pii/S1740674911000370.

[9] Sehlin D, Fang XT, Cato L, Antoni G, Lannfelt L, Syvanen S. Antibody-based PET imaging of amyloid beta in mouse models of Alzheimer/'s disease [Journal Article]. Nat Commun. 2016;7. Available from: http://dx.doi.org/10.1038/ ncomms 10759.

[10] Waldron AM, Fissers J, van Eetveldt A, Van Broeck B, Mercken M, Pemberton DJ, et al. In vivo amyloid-ßimaging in the APPPS1-21 transgenic mouse model with a 89Zr- labeled monoclonal antibody [Journal Article]. Frontiers in Aging Neuroscience. 2016;8. Available from: http: //www. frontiersin.org/Journal/ Abstract . aspx?s=18\&name=aging_neuroscience\&ART_DOI=10 .3389/fnagi . 2016.00067.

[11] Fissers J, Waldron AM, De Vijlder T, Van Broeck B, Pemberton DJ, Mercken M, et al. Synthesis and Evaluation of a Zr-89-Labeled Monoclonal Antibody for ImmunoPET Imaging of Amyloid- $\beta$ Deposition in the Brain [Journal Article]. Molecular Imaging and Biology. 2016;p. 1-8. 
[12] Krishnaswamy S, Lin Y, Rajamohamedsait WJ, Rajamohamedsait HB, Krishnamurthy P, Sigurdsson EM. Antibody-derived in vivo imaging of tau pathology [Journal Article]. The Journal of neuroscience : the official journal of the Society for Neuroscience. 2014;34(50):16835-16850. Available from: http: //europepmc.org/abstract/MED/25505335http://europepmc .org/ articles/PMC4261104?pdf=renderhttp://europepmc .org/articles/ PMC4261104http://dx.doi.org/10.1523/JNEUROSCI . 2755-14. 2014. 


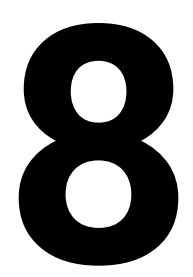

SUMMARY 
This thesis investigates the use of antibodies for the treatment and diagnosis of Alzheimer's disease (AD).

Chapter 1 gives a short historical perspective on the etiology and clinical features of $\mathrm{AD}$, while also providing an overview of the current diagnostic options that are now available.

In Chapter 2 we present an up-to-date literature review about the role of neuroinflammation in $\mathrm{AD}$ and the current state of the art of anti-A $\beta$ immunotherapy. We describe the interplay between microglia and complement, which together lead to an altered inflammatory status in $\mathrm{AD}$, and how they might play a role in the disease process. Next, we describe past and foreseeable future therapies making use of both passive and active immunization. Potential effector mechanisms are discussed summarizing findings from pre-clinical and clinical studies. A complete overview of all completed and ongoing human clinical trials is presented, showing that despite more than 25000 participating AD patients, still no therapy has reached clinical practice. Currently, most therapies are refocusing their target group to patients in the early stages of the disease, with the aim of preventing disease progression.

In Chapter 3, we investigated the effector mechanisms of $A \beta$ immunotherapy in a therapeutic and preventive fashion in the $5 \mathrm{xFAD}$ mouse model. To this end, an antiinflammatory (a modified mouse IgG1) and an inflammatory (mouse IgG2a) anti-A $\beta$ antibody (3D6) were administered to $5 \mathrm{xFAD}$ mice, either before (preventive treatment) or after (therapeutic treatment) the onset of behavioral symptoms and overt plaque pathology. Surprisingly, we found that antibody administration results in IgG mediated passive systemic anaphylaxis (PSA) caused by generation of anti-therapeutic antibodies (ATA). Preventive treatment was successful in reducing anxiety-like effects in 5xFAD animals. Our results also showed that mIgG 3D6 worsened memory problems in the contextual fear-conditioning test.

On a molecular level, we discovered that preventive treatment resulted in a stronger reduction on plaque load than therapeutic treatment. We also investigated the effects of immunotherapy on the different $A \beta$ fractions in brain homogenates (water soluble, detergent soluble and formic acid soluble $A \beta$ ). While therapeutic treatment lowered $A \beta$ in the detergent soluble fraction, preventive treatment on the other hand increased $A \beta$ in the detergent soluble fraction, caused by a shift from the formic acid soluble fraction to the detergent soluble fraction. No effects on microgliosis were observed, but our data showed that treatment with inflammatory antibodies (mIgG2a) significantly reduced astrogliosis. These results suggest an important role for astrocytes in the mediation of neuroinflammatory processes.

Given the observation that mIgG1 3D6 (anti-inflammatory) induced an isotype specific worsening of memory, while mIgG2a 3D6 (inflammatory) reduced astrogliosis, we suggest that isotype specific effects on inflammation need to be taken into account when designing immunotherapies for AD. Furthermore, our results suggest that a more pro-inflammatory therapy might be more beneficial for $\mathrm{AD}$ treatment.

Chapter 4 shows a proof-of-concept for the use of $A \beta$ antibodies for in vivo imaging of amyloid. For these experiments, an $A \beta$ specific antibody (bapineuzumab) was radiolabeled with a long-lived positron emitter $\left({ }^{89} \mathrm{Zr}\right)$ and compared with an isotype control (mAb 637) in the 5xFAD mouse model at several disease stages. The results 
showed that specific in vivo accumulation of antibodies can be detected using PET imaging. In addition, biodistribution experiments proved an age-related increase of ${ }^{89} \mathrm{Zr}$-bapineuzumab uptake in the brain. To further prove specificity, we could show that competition with unlabeled bapineuzumab reduced ${ }^{89} \mathrm{Zr}$-bapineuzumab uptake in the brain to background levels. No uptake of the isotype control could be found. Specific accumulation of administered antibody was further demonstrated by autoradiography and by colocalisation of administered ${ }^{89} \mathrm{Zr}$-bapineuzumab with plaques in the brain.

In Chapter 5 we compared different antibodies for their potential to use for in vivo PET imaging. Using 5xFAD mice of different ages, we now compared the in vivo binding properties of three $A \beta$ antibodies targeting monomers (solanezumab), oligomers (20C2), and plaques (bapineuzumab). Only ${ }^{89} \mathrm{Zr}$-bapineuzumab allowed for in vivo detection of plaques. With ${ }^{89} \mathrm{Zr}$-solanezumab and ${ }^{89} \mathrm{Zr}-20 \mathrm{C} 2$ no increased in vivo binding could be found. Biodistribution revealed that, as previously published, ${ }^{89} \mathrm{Zr}$-solanezumab does not seem to enter or remain in the brain in large concentrations. However, at 6 months of age, there was a significant accumulation of ${ }^{89} \mathrm{Zr}-20 \mathrm{C} 2$ in $5 \mathrm{xFAD}$ animals, which binds oligomers, in the brain. More interestingly, we found that at 3 months of age, there is a reduction of ${ }^{89} \mathrm{Zr}-20 \mathrm{C} 2$ binding in the cortex of $5 \mathrm{xFAD}$ animals compared to WT animals, a situation that is reversed at the later ages of 6 and 9 months. Analysis of antibody concentration in the blood also showed that there ${ }^{89} \mathrm{Zr}-20 \mathrm{C} 2$ was cleared more rapidly at 3 months of age in 5xFAD animals than in other conditions, a situation we did not observe with the other antibodies.

While ${ }^{89} \mathrm{Zr}$-solanezumab was cleared very rapidly from the animals (resulting in low concentrations in both 5xFAD and WT animals), we did find a specific accumulation of ${ }^{89} \mathrm{Zr}$-solanezumab in adipose tissue. Since only ${ }^{89} \mathrm{Zr}$-solanezumab binding is increased in adipose tissue in $5 \mathrm{xFAD}$ animals, it is likely that monomeric, soluble $\mathrm{A} \beta$ accumulates in fat deposits in the body.

Finally, we also showed that ${ }^{89} \mathrm{Zr}$-bapineuzumab accumulated in cervical lymphnodes of 5xFAD animals. This might be due to draining amyloid present in these lymphnodes, although it is more likely that there is an antibody specific mechanism, since the other antibodies did not show any accumulation in the lymphnodes.

In this work I present several advances using antibodies for both the diagnostic and therapy for AD. From a therapeutic point of view, I discovered that the effector mechanisms of an antibody need to be carefully considered to achieve a therapeutic effect. Furthermore, I showed that earlier treatment might be more beneficial than late treatment. In subsequent work, I explored the use of antibodies for imaging. While better techniques are available to image general amyloid load in the brain, we showed that using immunoPET imaging, we can obtain valuable information about antibody behavior in vivo and potentially also learn more about the $\mathrm{AD}$ disease process. 



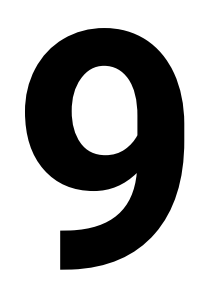

SAMENVATTING 
In deze thesis wordt het gebruik van antilichamen voor de behandeling en de diagnose van de ziekte van Alzheimer (AD) onderzocht.

In Hoofdstuk 1 wordt een kort historisch perspectief geboden over de etiologie en de klinische symptomen van $\mathrm{AD}$. Daarnaast wordt ook een overzicht gegeven van de huidige diagnostische technieken die ter beschikking staan.

In Hoofstuk 2 wordt aan de hand van een literatuuronderzoek de rol van neuroinflammatie in $\mathrm{AD}$ en de laatste stand van zaken met betrekking tot anti-amyloide immunotherapie beschreven. De interactie tussen microglia en het complement systeem, dat ervoor zorgt dat patiënten een verandering in de inflammatoire toestand ondergaan, werd uitgebreid beschreven. Daarnaast wordt dieper ingegaan op reeds ontwikkelde en toekomstige passieve en actieve immunotherapieën. Mogelijke werkingsmechanismen worden besproken aan de hand van pre-klinische en klinische studies. Verder wordt een volledig overzicht gegeven van alle klinische studies uitgevoerd bij patiënten tot nu toe. Hieruit blijkt dat ondanks het feit dat reeds meer dan 25000 patiënten deelgenomen hebben aan deze studies, er nog steeds geen therapie voor algemeen gebruik beschikbaar is. Op dit moment verleggen veel therapieën de focus naar patiënten in een vroeg stadium van de ziekte.

In Hoofdstuk 3 worden de resultaten beschreven van een onderzoek waarbij de werkingsmechanismen van $A \beta$ immunotherapie in een acuut en preventieve manier onderzocht zijn in het 5xFAD muismodel. Hiervoor werden een anti-inflammatoire (een gewijzigd muis IgG1) en een pro-inflammatoir (muis IgG2a) anti-A $\beta$ antilichaam (3D6) toegediend voor zowel een preventieve als ook een curatieve behandeling. Verassend genoeg vonden we dat toediening van antistof kan leiden tot anafylactische schok bij muizen, dat mogelijk veroorzaakt werd door het ontstaan van neutraliserende antistoffen. Preventieve behandeling was succesvol in het reduceren van angstig gedrag in 5xFAD muizen. Onze resultaten lieten ook zien dat geheugenproblemen erger werden in muizen behandeld met anti-inflammatoir antistof in de fear conditioning test. Op moleculair gebied, zagen we dat preventieve behandeling leidde tot een sterkere reductie van plaques dan de curatieve behandeling. We hebben ook het effect van immunotherapie op verschillende $A \beta$ fracties onderzocht in breinhomogenaten. Hierbij zagen we dat, afhankelijk van de behandeling, er een verschuiving van $A \beta$ plaatsvond. Daarnaast vonden we dat het inflammatoir antilichaam astrogliosis sterk kon reduceren.

In Hoofdstuk 4 lieten we zien dat het mogelijk was $A \beta$ antilichamen te gebruiken voor in vivo beeldvorming. Hiervoor werd bapineuzumab gelabeld met een langlevende positron emitter $\left({ }^{89} \mathrm{Zr}\right)$ en vergeleken met een isotype controle in verschillende stadia bij de 5xFAD muis. Dit experiment liet zien dat in vivo accumulatie van antilichaam in het brein gedetecteerd kon worden met behulp van een PET scan. Daarnaast liet de biodistributie zien dat er een leeftijd gerelateerde verhoging van opname is ${ }^{89} \mathrm{Zr}$ bapineuzumab. Dit was niet het geval bij de isotype controle. Specificiteit van de techniek werd verder aangetoond met autoradiografie en colocalisatie studies.

In Hoofdstuk 5 werden drie verschillende $A \beta$ antilichamen vergelijken met in vivo PET imaging. Deze drie antistoffen waren gericht tegen monomeren (solanezumab), oligomeren (20C2), en plaques (bapineuzumab). Alleen ${ }^{89} \mathrm{Zr}$-bapineuzumab kon in vivo plaques detecteren. ${ }^{89} \mathrm{Zr}$-solanezumab and ${ }^{89} \mathrm{Zr}$-20C2 lieten geen specifieke brein opname in vivo zien. Zoals andere studies ook lieten zien kwam ${ }^{89} \mathrm{Zr}$-solanezumab niet 
in het brein terecht. ${ }^{89} \mathrm{Zr}-20 \mathrm{C} 2$ liet een verhoogde opname zien vanaf 6 maanden in $\mathrm{AD}$ dieren, maar verassend was er een verlaagd opname van ${ }^{89} \mathrm{Zr}$-20C2 bij 3 maanden in $\mathrm{AD}$ dieren. Dit kan te wijten zijn aan het kortere half-leven van dit antilichaam bij 3 maanden. Interessant genoeg vonden we wel verhoogde concentraties van ${ }^{89} \mathrm{Zr}$-solanezumab in vetweefsel bij $\mathrm{AD}$ muizen, wat suggereert dat monomeer $\mathrm{A} \beta$ in vetweefsel accumuleert. Tenslotte lieten we ook zien dat ${ }^{89} \mathrm{Zr}$-bapineuzumab in de lymfknopen van de 5xFAD muis accumuleerde, wat erop kan wijzen dat $\mathrm{A} \beta$ van het brein hierin terecht komt.

Samenvattend presenteer ik verschillende nieuwe inzichten voor het gebruik van antistof in therapie en diagnose voor de ziekte van Alzheimer. Vanuit een therapeutisch perspectief, ontdekte ik dat de werkingsmechanismen van antilichamen beter onderzocht moeten worden om een therapeutisch effect te bereiken. Daarnaast liet ik zien dat een preventieve behandeling beter werkt dan een curatieve behandeling. Ook de mogelijke toepassing van antilichaam voor diagnose werden onderzocht. Hoewel het waarschijnlijk niet haalbaar is om deze techniek routinematig bij patiënten toe te passen, liet ik zien dat immunoPET nuttige inzichten kan opleveren over de werking van antistoffen in het lichaam en we zo ook meer kunnen leren over de ziekte van Alzheimer. 



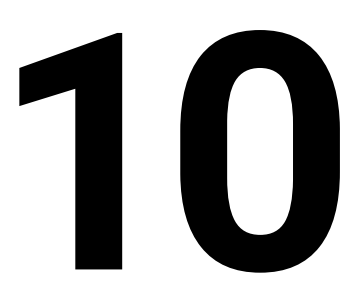

CurRiculum VitaE 
Jo Stevens was born on the 24th of May 1983 in Tongeren, Belgium. He obtained his high school degree in 2001 from the Onze-Lieve-Vrouwhumaniora in Tongeren. After this, he moved to Maastricht, Netherlands to study Knowledge Engineering. During this time he also was active in the faculty council, and followed a summer school at Baylor University, Waco, Texas. For his master thesis he developed a robotic goalkeeper under the supervision of Dr. Nico Roos. He obtained his Master degree from Maastricht University in 2006, with a major in Artificial Intelligence. Subsequently, he started studying Psychology and Molecular Life Sciences at Maastricht University in 2006. While studying these two programs, he was also active in the faculty council of the Faculty of Psychology and Neuroscience, and later as a board member from the same faculty. He also fulfilled various functions in the board of study association SV Helix. Meanwhile, he also worked as a software developer for UM Career Services from 2006 onwards. In 2008, he also received an appointment as lecturer at the Department of Knowledge Engineering for the teaching of the course Cognitive Psychology and Knowledge Presentation, which he taught for five years. In addition to this, he also served as a tutor for various courses such as programming and statistics at the Faculty of Psychology and Neuroscience. He received his Bachelor in Psychology in 2009. After this, he went on to study the Research Master Cognitive and Clinical Neuroscience, with a major in Fundamental Neuroscience in 2009. In 2009 he received an appointment as a research assistant at the School for Mental Health and Neuroscience. He was also active in the educational board of the Research Master. For his master thesis, he investigated the role of Dok7 in Myasthenia Gravis and the role of CERT in Alzheimer's disease. In 2011, he received a Bachelor in Molecular Life Sciences and a Master in Cognitive and Clinical Neuroscience. He subsequently got a job as a PhD student under the supervision of Prof. Dr. Marc de Baets, Dr. Mario Losen and Dr. Pilar Martinez-Martinez. During his $\mathrm{PhD}$, he was also active as $\mathrm{PhD}$ representative for PhD students of Division 3 in the Department of Neuroscience. The topic of his $\mathrm{PhD}$ was the investigation of antibodies for the therapy and diagnostics of $\mathrm{AD}$, the result of which is described in this thesis. He now work as a post-doc for Roche Diagnostics GmbH in Münich, Germany where he develops novel technologies for antibody generation. 


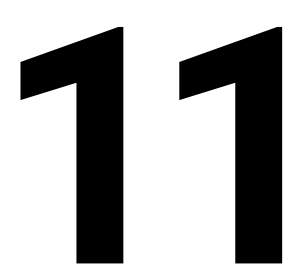

LIST OF PUBLICATIONS 


\section{PUBLISHED PEER-REVIEWED}

A. M. Gomez, J.A.A. Stevens, P. Molenaar, H. Duimel, F. Verheyen, J. Cossins, D. Beeson, M.H. De Baets, M. Losen, P. Martinez-Martinez, Silencing of Dok-7 in adult rat muscle increases susceptibility to passive transfer myasthenia gravis, American Journal of Pathology, In press.

P. Martinez-Martinez, P.C. Molenaar, M. Losen, C. Hoffmann, J.A.A. Stevens, L.D. de Witte, T. van Amelsvoort, J. van Os, B.P.F. Rutten, Auto-immune disorders as a possible cause of neuropsychiatric syndromes, Tijdschrift voor Psychiatrie, 2015. [Dutch]

K. Vrolix, J. Fraussen, M. Losen, J.A.A. Stevens, K. Lazaridis, P. C. Molenaar, V. Somers, M. A. Bracho, R. Le Panse and P. Stinissen, Clonal heterogeneity of thymic B cells from early-onset myasthenia gravis patients with antibodies against the acetylcholine receptor, Journal of Autoimmunity, 2014

J.A.D. Dela Cruz, R. Schmidt-Kastner, J.A.A. Stevens, H.W.M. Steinbusch, B.P.F. Rutten, Differential distribution of hypoxia-inducible factor 1-beta (ARNT or ARNT2) in mouse substantia nigra and ventral tegmental area, Journal of Chemical Neuroanatomy, 2014

Martinez-Martinez P, Molenaar PC, Losen M, Stevens JAA, Baets MH, Szoke A, Honnorat J, Tamouza R, Leboyer M, Os JV, Rutten BP, Autoantibodies to neurotransmitter receptors and ion channels: from neuromuscular to neuropsychiatric disorders, Frontiers in Genetics, 2013

\section{SUBMITTED}

J.A.A. Stevens, P. Martinez-Martinez, L. Dubois, D.J. Vugts, T.E. Nell, S.M. Crivelli, W.A. Buurman, P.C. Molenaar, B. Brans, P. Jelle Visser, M. de Baets, G.A. van Dongen, M. Losen. In vivo PET imaging using an anti-amyloid antibody in an Alzheimer's disease model.

J.A.A. Stevens, A. Franssen, S.M. Crivelli, M. de Baets, P. Martinez-Martinez, M. Losen. Isotype dependent effector mechanisms of immunotherapy in Alzheimer's disease.

J.A.A. Stevens, M. Losen, M. de Baets, P. Martinez-Martinez. Inflammation and Alzheimer's disease: lessons from immunotherapy.

I. Koneczny, J.A.A. Stevens, A. de Rosa, S. Huda, M. G. Huijbers, A. Saxena, M. Maestri, K. Lazaridis, P. Zisimopoulou, S. Tzartos, J. Verschuuren,S.M. van der Maarel, P.van Damme, A. Vincent, M.H. de Baets, P.C. Molenaar, R. Ricciardi, P. Martinez-Martinez, M. Losen. IgG4 autoantibodies against muscle-specific kinase undergo Fab-arm exchange in myasthenia gravis patients.

A. Saxena, J.A.A. Stevens, H. Cetin, I. Koneczny, R. Webster, K. Lazaridis, S. Tzartos, K. Vrolix, G. Nogales Gadea, P.C. Molenaar, J. Damoiseaux, M. H. De Baets, A. Marx, A.Vincent, M. Losen and P. Martinez-Martinez. Characterization of an anti-fetal AChR monoclonal antibody isolated from a myasthenia gravis patient.

\section{IN PREPARATION}

J.A.A. Stevens, P. Martinez-Martinez, L. Dubois, D.J. Vugts, T.E. Nell, S.M. Crivelli, W.A. Buurman, M. de Baets, G.A. van Dongen, M. Losen. Anti-amyloid antibody 
characterization with immuno-PET.

C. Hoffmann, J.A.A. Stevens, M. Losen, P. Molenaar, M. de Baets, B. Rutten, J. van Os, P. Martinez-Martinez. Analysis of auto-antibodies targeting the alpha7 acetylcholine receptor in schizophrenia.

R.J.G. Gentier, J.A.A. Stevens, L.M. Verheijen, C.D. van ‘t Hekke, D. van den Hove, D.H.J.P. Hermes, H.W.M. Steinbusch, J.J. Cheng, M.O. Grimm, V. J. Haupenthal, W. Annaert, T. Hartmann and F.W. van Leeuwen, Paradoxical effects of mutant ubiquitin and $\gamma$-secretase during modelled Alzheimer's disease neuropathogenesis. 



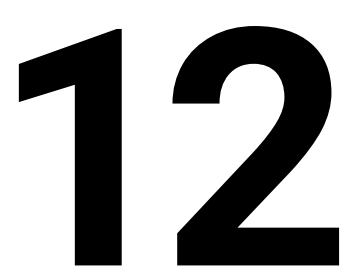

ACKNOWLEDGEMENTS 
Zoals de meeste lezers ongetwijfeld weten, heb ik reeds een lange carriere in Maastricht erop zitten. Dit druk belezen hoofdstuk is dan ook de perfecte plek om alle mensen te bedanken die rechtstreeks hebben bijgedragen tot mijn $\mathrm{PhD}$, maar daarnaast ook iedereen (of toch een poging tot), die gedurende mijn vele jaren hier, Maastricht tot een echte thuis voor mij hebben gemaakt.

Prof. de Baets, beste Marc, dank u wel voor alle steun en vertrouwen van de afgelopen jaren. Dankzij uw heb ik niet alleen over Alzheimer, maar ook over immunologie en Myasthenie heel erg veel bijgeleerd. Niet alleen wetenschappelijk bent $\mathrm{u}$ bron van inspiratie, maar ook als persoon heb ik het altijd goed met $u$ kunnen vinden. Onze lunches en conversaties over o.a. "'het thuisland"' waren altijd een welkome afwisseling van het werk.

Beste Mario, waar te beginnen... mijn eerste echte kennismaking met $\mathrm{u}$ was onderweg naar Rijswijk, waar ik letterlijk als proefaap moest fungeren. Nooit had ik kunnen denken dat dit zou uitmonden in zo een intense, lange samenwerken en er zouden nog vele lange dagen met elkaar volgen. Samen op congress naar Zweden en de VS, tot diep in de nacht EMG metingen doen en spieren snijden, en de vele marathon sessies bij de PET scanner in ons "tweede kantoor". Misschien tot de verbazing van sommigen heeft onze samenwerking wel altijd gewerkt. Ik denk dat dit komt door de vele gemeenschappelijke interesses die we hebben: we hebben allebei een affiniteit met technologie, houden van knutselen en klussen, en we houden er allebei van om na te denken over complexe problemen en nieuwe, niet altijd voor de hand liggende dingen, uit te proberen. Bedankt voor alle begeleiding, advies, en vooral de kans om zoveel verschillende en nieuwe dingen te leren en uit te proberen, de ene keer met wat meer succes dan de andere.

Dear Pilar, without you there would be no thesis and it would definitely not be finished as fast as it was. You managed to bring the necessary focus and motivate me to go the extra mile. You were the perfect person to complete this team, bringing the necessary focus and coming up with excellent suggestions for experiments and explanations. I am extremely grateful for all the efforts you spend in guiding me and correcting my writing, especially the last months. I still do not know how you manage to organize all the ongoing projects, answering everybody's questions, and raising a large family.

Alejandro, it seems a lifetime ago that I started my internship as your bachelor student. I still remember my first day, isolating bone marrow for the bortezomib experiment. My internship would be about silencing Dok7, and finally, after 6 years we even managed to publish it! You gave me the training I needed in molecular biology and cell culture, and also during my PhD I kept learning from you. More importantly, you became a valued friend and I was lucky enough to share some of the wonders of the world with you, such as the Northern lights and the Stromboli volcano. In the end, I even had the honor of being your paranimf.

Beste Gerard, je bent een wetenschapper in hart en nieren. Vaak lijk je een beetje verstrooid, of stil, maar diegenen die je beter kennen weten dat het gewoon nog te vroeg is op de dag om een antwoord te verwachten ;). Hoewel je thesis over dat bepaalde eiwit gaat, denk ik soms dat je hem beter had geschreven over de kunst van het koffiebrouwen! Jouw koffie is dan ook een ervaring waarvan ik het privelege gehad heb hem te mogen 
drinken, en waarom zou iemand naar de Beluga willen gaan als hij bij jou kan komen eten? Daarnaast delen we ook nog een passie voor de betere (Belgische) bierbrouwsels. Het was een hele eer toen je mij als paranimf vroeg, en het is dan ook met grote spijt dat ik er niet bij kon zijn op die geweldige dag.

Liefste Kathleen, als de andere Belg in ons groepje hebben we het altijd goed met elkaar kunnen vinden. Onze tijd samen in het lab was veel te kort, maar gelukkig hebben we elkaar nog vaak genoeg gezien toen je klaar was. Het voordeel van jou vertrek was natuurlijk dat ik je bureau kon inpalmen ;). Dankzij jou love voor "Frietjes met zoervlees" en andere carnavalhits hebben we vele leuke tijden samen beleefd. Ik kijk er naar uit om nog vele carnavalhits met jou, Vincent, en Bas, samen te blèren.

Chiarina!!!!!!!! Thank you for being you! You were the joy in our office with your chaotic, Italian ways, and your ability to make a little cave everywhere. I truly had fun with you, not only during work, but also after. Since you left, Maastricht has never been the same, and they even closed down your beloved V\&D, something I jokingly said when you were leaving, but never thought would really happen. We still need to arrange a date when Kevin is away to put that delicious Monty I the shower :D

Lieftse Anne-Hilde, hoewel maar kort in onze groep, was je altijd een wezenlijk onderdeel van het originele "Neurimclubje". Het was dan ook superleuk dat je later weer terug op onze afdeling rondliep (hoewel ze je kantoor ver weg van het mijne hadden verstopt). Dank je wel voor alle leuke tijden samen, en altijd een inspanning deed om samen met ons het evenement van het jaar tenminste 1 dag te vieren :D

Dear Carolin, nach soviele Jahren weet ik nog immer nicht which language ich met jou sprechen sollte. After a short break after you bachelor, we end up (again) in the same group. You managed to break the spell of the cursed desk in our office, and are almost finishing your PhD as well. Throughout the years, we not only became good colleagues that worked together on various projects, but more importantly good friends. We had tons of fun at various parties and soon you will even close (again?) your favorite place in the whole world, the Alla :D. I am really happy that you wanted to be my paranimf, and I look forward to coming to your defense!

Dear Simone, I still remember your enthusiasm in the "antibody production" practicum I gave during FN. Little did I know that you would end up as a student in our group, and maybe it was not official, but I still count you as one of my students ;) I will never forget all the hours in that DM3 room chasing after those horrible animals. I am also happy that we became friends during this period and could bond over several beers. Besides starting a PhD with us after your internship, you also started a family, and I wish you, and your family the very best for the future!

Ai Marina, you really livened up the office in my final year. Despite the fact that you like salsa, and that all the student questionnaires say differently, you are still a very nice person. I think you are the perfect follow up for the "angry person" in our office, you only need to practice in watering the plants... Always keep your energy and good spirits, and you will do just fine.

Shengua!! Zo ta ma!!!!! Luckily, only few people will understand that sentence ;). You were my second close encounter with Chinese culture, and you really learned me a lot, especially with our Chinese language lessons ;). You are an amazing, intelligent person, and it was a real joy to work with you. More importantly, you became a good friend, 
always willing to offer assistance when needed. I place the important responsibility of watering the plant to you, because I know Marina won't take care of it. As a reward, I give you custody over my desk chair, take care of it! (In addition, the post card wall is lacking cities from China, so it will be your responsibility to fill it up).

Shannen, het was een genot je in onze groep te mogen verwelkomen. Hopelijk zien we elkaar nog vele malen op kerstdiners, of basement feestjes.

During my time in our group, I also had the pleasure to learn from a number of postdocs. Prashant, you (or actually I think your wife) showed us the meaning of good Indian cuisine. In addition, I learned some valuable lessons about protein purification from you. Chèr Marjorie, notre temps était court, mais j'ai apprend un motton de coses de toi. En plus, la tarte au poir était le plus bon que j'ai mangé en tous cas. Dear Abhishek, you showed me that it is possible to make a career out of antibodies. I really enjoyed working with you and I learned a lot. Gisela, gracias por venir a Maastricht y por tu ayuda y consejos en tantas cosas, no solo científicos, pero también en los consejos de la vida ;) Buena suerte con tu propio grupo y nos veremos pronto en Badalona! And then there is Inga... ;) Sometimes, I wonder if you ever sleep, because you are always so full of energy and enthusiasm, it is impossible for me to imagine you being quiet. Still, your energy and curiosity work infectious, and you never ceased to amaze me with your seemingly endless knowledge. Beste Peter, dank je wel voor al je wijze inzichten over spieren en het eindeloze editen van mijn manuscripten.

Beste Prof. Buurman, beste Wim. In een ver verleden hebben we mekaar ontmoet tijdens mijn bachelor in een majorblok. U was de eerste en enige docent die geen powerpoint gebruikte, maar nog gewoon een echt blackboard, en was voor mij een inspiratie om in de wereld van immunologie verder te gaan. Dank u wel voor de inspirerende gesprekken over wetenschap, het immuunsysteem, geduldig mijn schrijfstijl verbeteren, en de anecdotes over uw vele reizen.

Deze thesis kon nooit tot stand zijn gekomen zonder mijn studenten. Sofie, zoveel tijd dat we in het RNL hebben doorgebracht... Je was een perfecte, hardwerkende student, en van jouw werk plukken nu nog PhD studenten de vruchten. En, je bent er zelfs in geslaagd jouw scriptie voor mijn thesis in te leveren ;). Aimee, wat zou ik zonder jou gedaan hebben! Je hebt twee stages bij mij gelopen, en daar ben ik heel blij om. Samen hebben we heel veel geleerd over dierexperimenten en werk organiseren. Zonder jouw hulp, was ik nu waarschijnlijk nog met de PhD bezig. Heel veel succes met je eigen doctoraat in Schotland! Thanks also Manuel for the help with the ELISAs and good luck with your career!

Although I worked very closely together with the people in my research group, I could never have learned so much and developed into the person that I am today without all the other people I got to know during my $\mathrm{PhD}$.

Beste Fred, als er één iemand een wetenschapper pur-sang is, dan bent $u$ het wel. Als de Alzheimer autoriteit op onze afdeling ben ik $u$ eeuwig dankbaar voor alles wat ik van $u$ heb kunnen leren. $U$ heeft mij altijd onder uw hoede genomen op o.a. SFN en de $\mathrm{AD} / \mathrm{PD}$, en ook de kans gegeven om labs zoals deze van de Mandelkows te leren kennen. Ik heb genoten van onze discussies over de rol van het proteasoom en gamma-secretase, maar $\mathrm{u}$ was evenzeer te vinden voor het bekijken van WK matchen. Over 2 jaar bent $\mathrm{u}$ van harte welkom in München zodat we samen kunnen kijken hoe België Duitsland (of 
Nederland?) verslaat in de final.

Jos, het was een beetje een sprong in het diepe toen ik met 6 anderen begon aan de master FN. Voor mijn persoonlijke situatie was het echter de perfecte keuze, en dat eerste jaar was dan misschien wel een experiment, maar wel een succesvol experiment. Ik ben dan ook samen met $\mathrm{u}$ supertrots dat FN nu zo een succes is. Daarnaast ben ik $\mathrm{u}$ dankbaar om mij te laten kennismaken met onderzoek in de industrie.

Beste Govert, dank u wel voor alle leuke conversaties (wetenschappelijk en niet wetenschappelijk) die we over de jaren heen hebben gehad. U bent één van de oprechtste mensen die ik ken, en ik heb het een eer gevonden met $u$ te mogen samenwerken.

Daniel en Gunter, voor mij zijn jullie als twee verschillende kanten van een munt, maar dan wel eentje van veel waarde. Toen ik het befaamde blok 2.1 zelf volgde, wist ik, door jullie passie voor onderwijs en uitleg, dat ik in neurowetenschappen verder wou. Later heb ik jullie beiden leren kennen als uitstekende onderzoekers en ook nog eens als geweldige personen.

Prof. Steinbusch, dank $\mathrm{u}$ wel voor uw ondersteuning en interesse in mijn werk. Het was altijd aangenaam wetenschappelijke discussies met $u$ te hebben, en ook meer inzicht te krijgen in de politiek achter de wetenschap.

I would also like to thank the other seniors in our department, Bert, Jassin, Bart, Marlies, Tatyana, Peter and Ali, thank you for your interest. Een speciaal dank je wel is voor de volgende dames: Lisa, Rachelle, Marie-Therese, Peggy, Ankie, en Nicole, die in hun verschilende functies altijd gezorgd hebben dat alles vlotjes verliep.

Mijn PhD was nooit mogelijk geweest zonder ons uitstekende team van technicians. Dank je wel Hellen voor alle adviezen en hulp over de jaren. Barbie, Marjan, Denise en Marcella, zonder jullie inzet was ik ongetwijfeld nog breintjes aan het snijden en kleuren, bedankt voor alle hulp en adviezen! Ook een grote dank aan Wiel, voor de leuke babbels en alle hulp met de dieren.

Off course there were also a lot of other people in my department who I am extremely grateful. A big thank goes to the assistant, soon to be professor, Joao. I think one of the first times I met you was when you were giving a presentation in the pain-course of FN and you had an original idea that you wanted to let the rats exercise more. You are an inspiring person, a good scientist, and a great cook. Thanks for the Portuguese dinners, keeping my company in the basement, "fun-with-the-nanodrop"-part 1 and 2, and our adventures in the city. It means a great deal to me that you accepted being my paranimf and I wish you the very best in your new position in Lisbao.

Then there is whole list of people in our department to whom I own thanks for great times during and after work. To "the guys": Koen, Maarten, Roy, Ehsan, and Pim, what happens in Berlin stays in Berlin. I had a great time with you guys, and I hope that many more trips will follow. Sven, our thirtieth Birthday party was one of the epic parties in the history of Maastricht that people are still talking about years later, thanks for hosting ;). Lisa, Rianne, and Nicole, my FN buddies in the department, we followed a long way together, but now it seems our ways are finally parting.

Merci à les filles, Laurence, Celine, Melanie, et Marion, grâce à vous j'ai peux pratiquer mon français pendant les conversations très agréables. Artemis, thanks for your uplifting spirit and your ability in bringing people together. Yuan, you were my 
first real introduction to Chinese culture, and I always enjoyed your company. I was really honoured that I could be your paranimf. Thanks to all the other people in the department: Mark, Sarah, Fred, Youssef, Remco, Paul, Dagmar, Nynke, Nick, Brit, Sandra, Ramona, Romina, Frank, Majed, Anne, An, Lianci, Evi, Birgit, Gusta, Jasper, Fabien, Pablo, Leonidas, Maria, Daan, Annerieke, and Eva. I had some great times with all of you, from a dinner, labday out, parties, a night in the Alla, trips to Silicy or conferences in Washington or Nice, making chordomas, spending time in the basement, or even remodelling an entire apartment (although you repaid with lots of cake and lunches Ramona ;)).

Julie, sometimes I cannot believe that we used to work together, because it feels such a long time ago. So many things we did together, from being at the uni every weekend to going to Oktoberfest. Your choice in paranimfs was like a prediction of things to come, and I thank you that you choose me for being there on that day. I look forward to being there on your next special day, and I am already practicing;).

Een bijzondere dank gaat naar de Nucleaire geneeskunde groep van het VUmc. Beste prof. Van Dongen, Danielle en Thijs, de helft van dit boekje is dankzij jullie tot stand gekomen. Bedankt voor de prettige en succesvolle samenwerking de afgelopen jaren. Ich wollte auch gerne das Team von dem Fraunhofer Institut in Aachen bedanken. Danke dr. Fendel und Herr Rosinke für alle Hilfe und die Möglichkeid unsere Antikörper bei Ihnen herzustellen.

Ook binnen de UM zijn er heel wat mensen die mijn dank verdienen. Dank aan prof. Hoet voor zijn advies en inzichten over antilichaam productie en aan Mat Rousch voor hulp bij cloneren. Aan iedereen die ooit op een labhelp gereageerd heeft ;)

Verder gaat een speciaal dankwoord aan de medewerkers van het CPV. Beste Richard, Clarice, Saskia, Inger, Rik, Harry, Nathalie, Pauline en alle andere medewerkers. Het waren complexe projecten met veel dieren, maar dankzij jullie deskundige ondersteuning is alles tot een goed einde gebracht. Ik vond het heel fijn samenwerken, niet alleen op, maar ook naast het werk. Daarnaast wil ik ook Marjanne Quaaden bedanken. U heeft niet de meest eenvoudige job, maar altijd (weekend of in de VS maakt niet uit) staat $u$ klaar om onderzoekers te helpen en oplossingen te vinden voor laatste minuut wijzigingen.

Beste Marjanne Markerink en Natsaja, ik denk niet dat er veel PhD studenten zijn die zoveel met jullie te maken hebben gehad, van ontploffende fermentors tot virus injecties, ik ben het hele lijstje met jullie doorgegaan. Ik kon me echter geen betere personen indenken om alle problemen en vragen op te lossen.

Beste Sandra en Martine, nooit had ik gedacht dat ik tijdens mijn PhD zoveel tijd een verdieping lager bij jullie zou doorbrengen. Van de vele RIAs tot de vele PET scans, het RNL voelde soms als een tweede thuis. Dank jullie wel voor de fijne samenwerking.

Daarnaast wil ook Ludwig Dubois bedanken voor de hulp met mijn PET experimenten. Verder verdienen ook de mensen van Nucleaire Geneeskunde van het aZM een bijzondere vermelding. Dank je wel Christian, Roel, dr. Brans, Matthias en Geert voor alle hulp en samenwerking met de PET scanner. Beste prof. Hoet, dank u wel voor al uw hulp en advies tijdens mijn antilichaam productie problemen. Zonder dit, was dit project nooit gelukt. Daarnaast wil ik u bedanken voor de adviezen over werken in de industrie. 
Gedurende de jaren voor en tijdens mijn $\mathrm{PhD}$ heb ik ook van vele mensen hulp gehad. Graag wil ik mijn oude M.Sc. begeleider, dr. Nico Roos, bedanken om mij te mogelijkheid te geven les te komen geven in mijn oude opleiding. Het was een vrij unieke situatie dat ik nog als student een eigen vak gaf, maar ik kwam met ieder jaar weer met plezier terug naar mijn oude thuis . Ook dank aan Jan voor alle de leuke babbels en biertjes, het pensioen komt er aan Jan ;) En daarnaast natuurlijk dank aan alle andere mensen bij DKE die mij ondersteunt hebben, Karin, Gonny, Ralf, Jos, en Frank, het was heel leuk om na mijn studie terug met jullie samen te werken.

Verder gaat wil ik ook het hele team van Studentenbegeleiding bedanken voor de fijne jaren dat we samengewerkt hebben. In de eerste plaats aan Heiny Eilkes, om mij een kans te geven toen UM Career Services nog een idee op papier was. Vanuit het niets is er door de jaren heen een prachtig project gegroeid dat nu als een vaste waarde in de UM functioneert. Daarnaast ook natuurlijk een bijzondere dank aan Ester en verder aan Monique, Caroline, Anita, Floor, Greet, Jose, Karin, Koannie, Lily, Maddy, Marianne, Marlies, de Miekes, Peter, Robbie, Tessa, en Wendy. Samen hebben we een mooie afdeling op touw gezet, en ik ben trots dat ik ook mijn steentje kon bijdragen.

Ik heb vele bijzondere dingen mogen beleven aan de UM, eentje daarvan was toch zeker mijn tijd in het bestuur van FPN. Dank aan Bernadette, Paul, Madelon, Arie en mijn studentcollegas Marjolein en Anouk voor deze leuke ervaring in een faculteit achter de schermen. Een bijzonder woord gaat naar Trudy, die mij gedurende mijn hele studententijd is blijven achtervolgen ;)

Mijn mede-KTers, we zijn nu een letterlijk over de hele wereld verspreid, maar wat een leuke tijd hebben we beleefd, en beleven we nog steeds samen, helaas niet genoeg. Dank Berend, Laurens, Tjebbe, Timor, Rick, Kitty en Bernard voor de leuke tijd samen. Berend, je zult toch nog eventjes met Juliana en Scarlet in Australie moeten blijven tot ik ben langsgekomen. Laurens, groot was mijn verbazing toen ik langgeleden je kenmerkende MSN profielfoto zag bij Danique op het scherm, toen nog een medestudente in mijn eerste onderwijsgroep bij psychologie ;). Ik hoop jullie snel weer te zien, en succes in NYC! Ook aan alle andere KuT studenten, in bijzonder Boris, Victor, Driek, Maarten(s), Daan, Nyree, Maurice(s), en Guus, het was een mooie tijd!

Aan de Helix-mensen, wat een geweldige jaren was dat bestuur. Samen hebben we Helix op de kaart gezet, dus dank aan Timo, Liesbeth, Siamack, en Jennifer. Guido, Marjolein, Maarten (en Vicky), Dennis (en Nadja), Evelien en Marijke, we waren niet alleen bij Helix actief, maar vooral ook daarbuiten. We hebben vele leuke dingen samen beleefd, en ik hoop dat er nog vele mogen volgen. Succes met het afmaken van jullie eigen PhD Guido, Marjolein en Dennis. Maarten en Marijke, succes met postdoccen in Zweden en in Maastricht ;).

Und dann die Mensachiller crew! Ihr alle habt ein großes Danke schon verdient. Ihr besondere deutsche Unterricht hat am Ende funktioniert, und es ist unglaublich, dass ich jetzt selber in Deutschland arbeite. Wir haben so viele krasse Geschichte zusammen erlebt, darüber könnte ich noch 1 oder 2 Buchen mehr schreiben. Weil wir sehr viele tolle Sachen erlebt haben, glaube ich das die stärkste Verbundenheit mit uns kommt, durch, dass wir das schlimmste was passieren kann, zusammen mitgemacht haben. Wir trinken eine auf Stebbe! Danke Horst, Niklas, Thomas, Chris, Dima, Dave, Mr. T, Marcose, und Kevin für Ihre Freundschaft. Kevin und Caro, danke für die Gesellschaft in Maastricht, 
das gute Essen, und zusammen mit Dave unsere Suits und Game of Thrones Abends.

Aan alle oud-collega's van het Repenhof, in het bijzonder aan Hans, Svea, Julie, Marjan en Kathy die ik nog regelmatig tref, dank voor de leuke jaren samen.

Geert, Gijs, Lindy, Ellen, Stephanie, Evi en Carl, zovele jaren vriendschap en hopelijk komen er nog vele jaren bij. Dank jullie voor de leuke jaren en feesten samen.

Nienke y Carlos, soy muy feliz por tener vuestra amistad. Muchas gracias por estos últimos tres años tan divertidos. Hemos hecho fiestas en toda Europa y al final fuimos a Tomorrowland :D. Además, gracias en gran parte a vosotros, ahora se Español :P Espero que nuestra amistad continues por mucho años más.

Gracias a todo el grupo latino/español/Nederlands;) de Maastricht/Aachen/Koln/Utrecht: Jaime, Remon, Miguel Angel, Dani, Karim, Juanmi, Cris (plural), Santi, Andrea y Peter. Ahora conozco mucho mejor el mundo gay ;). También a toda la gente en Munich, Bernardo, Salvatore, Sarah, Stephan,Livia, Lidia, Jorge, Alberto, Joanna, David, Tomomi, Ricci, Edgar, Marcia, Frank, Gisela, David, Luis, Mariela, Papi, Rocío, Marcos y Giselita. Os conozco a vosotros gracias a Yara, pero la vida en Munchen es mucho más fácil, sabiendo que tengo cerca bueno amigos.

I am grateful for all the other friends I met in Maastricht. Thanks International Dinner club, Mehrdad, Sanne, Joost, Thessely, Martin, Paula, Giacomo, Jessie, Gabri, Nevena, Elisa, and Allison (even though I never had those burgers you promised). Martin and Thessely, you are always welcome to bring Toby to Munich for babysitting. Paulaner, I will be waiting with little patience for that invitation we talked about with a beer of your namesake in hand. Mehrdad, remember me when you have taken over Maastricht, and later on the world. Your caliphate will change the world for the better. Thanks people from the German/Dutch Dinner club, Elke, Jan, Annette, Carla, Tammy and Charlotte for the nice evenings together.

Vero, Cris, y Alba, nunca os he visto a vosotra tres junta, pero siempre es un fiestón cuando nos vemos. Marta y Lina, muchas gracias por los momentos tan divertidos siempre en Cambrils Resort. Nos vemos pronto!

Silvia, Victor, Luisito, y Irina, muchas gracias por los buenos ratos y lo bien que lo pasamosel cada vez que voy a Reus. Ha sido un complot! Gracias a Consuelo, Xavier, Aida, y Luis lahacerme sentir como uno más de la la familia. Siempre me siento como un rey en un hotel de cinco estrellas cuando vamos y me alegro de volver cada vez:).

Aan mijn familie, Erwin, Fabienne, Noel, Gina, Germain, Rita, Vital, Cristel, Danny, Myriam, Didier, David, Sylvia, Linda, Kris, Brecht, Leen, Elke, en Helena bedankt voor de steun. Ook wil ik Nicole en Daisy bedanken voor de steun.

Aan bobonne en ma, helaas zijn boupa en bou niet meer hier om dit mee te maken, maar ze staan wel allebei op verschillende pagina's in dit boek en prominent op de voorkant. Dank jullie voor jullie steun tijdens mijn lange tijd aan de universiteit.

Dank aan mijn broers Tim en Hanna, Wim en Astrid voor jullie steun. Misschien zal het niet het eerste boek zijn dat hij leest, maar ik ben er zeker van dat kleine Mats dit als tweede boek kan lezen ;).

Aan mijn ouders, dank jullie wel voor jullie steun tijdens deze vele jaren. Jullie hebben mij altijd de vrijheid gegeven te doen wat ik wou doen, waarvoor ik jullie heel dankbaar ben, ook al heb ik misschien niet altijd de makkelijkste weg gekozen.

Hola guapa! Now I know why loved ones are always last in the list, because it is the 
most difficult part to write... I am so happy I met you, it is very difficult to put in words. You were the light in the last years of my PhD. First as a friend to have a great time with and go on trips, and later on as much more. I still remember that moment when you were telling that for you it was impossible to find someone in the pueblo ;P, and look where we are now. I never expected or wanted to start something with a colleague, but the magic of the Alla works in mysterious ways ;). Thank you for all your support, your help, optimism, and being there these last years, and especially these lasts months, to give me the energy to go on and finish the lasts parts. I admire your perseverance and you are my inspiration. I cannot believe that I am in your beloved Munich now, while you are still there, but I am looking forward to the day you join me here and we continue building our life and a wonderful future together. 